\title{
Novel Therapeutic Approaches to Overcome Acquired Resistance to Enzalutamide in Patients with Advanced Prostate Cancer
}

Keith Thomas Schmidt 
Print: Ridderprint | www.ridderprint.nl.

ISBN: 978-94-6416-163-2

Copyright: K.T. Schmidt, Bethesda, Maryland, USA. 2020

All rights reserved. No part of this publication may be reproduced, stored in a retrieval system, or transmitted by any form or any means, mechanically, by photocopying, by recording or otherwise without the prior permission of the author. 


\section{Novel Therapeutic Approaches to Overcome Acquired Resistance to Enzalutamide in Patients with Advanced Prostate Cancer}

Nieuwe therapeutische benaderingen om verworven resistentie tegen enzalutamide te overwinnen bij patiënten met gevorderde prostaatkanker

(met een samenvatting in het Nederlands)

\section{Proefschrift}

ter verkrijging van de graad van doctor aan de

Universiteit Utrecht

op gezag van de

rector magnificus, prof.dr. H.R.B.M. Kummeling, ingevolge het besluit van het college voor promoties

in het openbaar te verdedigen op

dinsdag 6 oktober 2020 des middags te 12.45 uur

\section{Keith Thomas Schmidt}

geboren op 4 augustus 1989

te New Hyde Park, New York, USA 


\section{Promotor:}

Prof. dr. A.D.R. Huitema

\section{Copromotor:}

Dr. W.D. Figg 


\section{Table of Contents}

Chapter 1 - Introduction

Chapter 2 - Combatting Resistance to Second-Generation Androgen

Receptor Antagonists: Clinically Relevant Biomarkers and Novel Treatment Strategies in Metastatic Prostate Cancer

Chapter 3 - A Phase 2 Study of Seviteronel (INO-464) in Patients With 55 Metastatic Castration-Resistant Prostate Cancer After Enzalutamide Treatment

Chapter 4 - Anti-tumor Activity of NLG207 (formerly CRLX101) in 89 Combination with Enzalutamide in Preclinical Prostate Cancer Models

Chapter 5 - Measurement of NLG207 (formerly CRLX101) Nanoparticlebound and Released Camptothecin in Human Plasma

Chapter 6 - Population Pharmacokinetic Analysis of Nanoparticle-bound and 161 Free Camptothecin after Administration of NLG207 in Adults with Advanced Solid Tumors

Chapter 7 - NLG207 (formerly CRLX101) in Combination with 193 Enzalutamide in Patients with Metastatic Castration-Resistant Prostate Cancer Previously Treated with Enzalutamide

Chapter 8 - Summary

Hoofdstuk 8-Samenvatting

Chapter 9 - Discussion

Acknowledgements

Curriculum Vitae

Publications 
Chapter 1

Introduction 
Enzalutamide is a second-generation androgen receptor antagonist (SG-ARA) used as a standard of care treatment option for patients with metastatic castrationresistant prostate cancer (mCRPC). ${ }^{1,2}$ With improvements to on-target potency and capability to disrupt androgen receptor (AR) functionality in comparison to firstgeneration agents, enzalutamide significantly improved clinical outcomes for patients with mCRPC and offered an alternative to chemotherapy. ${ }^{3}$ The SG-ARA class has recently expanded to include apalutamide and darolutamide, while increasing clinical utility via treatment indications for patients with metastatic castration-sensitive prostate cancer (mCSPC) and non-metastatic castration-resistant prostate cancer (nmCRPC). ${ }^{4}$

Despite these important advances in prostate cancer drug development, the durability of SG-ARA therapy is limited by numerous mechanisms of acquired resistance..$^{5,6}$

Robinson et al. first characterized common alterations providing potential mechanisms of acquired resistance to SG-ARA therapy, demonstrating prominent tumor heterogeneity present in the advanced prostate cancer population. ${ }^{7}$ Studies that followed have served to confirm the roles of diverse clinical biomarkers in the propagation of unattenuated disease progression, while also motivating the clinical development of numerous agents to optimize therapeutic options and improve clinical outcomes.

Notable mechanisms of acquired resistance include alterations to the AR pathway (e.g. AR-V7 expression, AR overexpression) and hypoxia-mediated metabolic signaling pathways (e.g. WNT/ $\beta$-catenin, PI3K/AKT). ${ }^{7-9}$

The aim of this thesis is to facilitate the development of novel therapeutic approaches targeting clinically relevant mechanisms of enzalutamide acquired resistance 
to improve outcomes in patients with $\mathrm{mCRPC}$ via the use of applicable preclinical models, pharmacokinetic tools, and biomarker-driven clinical trial design.

This research was initiated following identification of viable drug targets and potential avenues of therapeutic development to overcome SG-ARA acquired resistance. ${ }^{7,10,11}$ Relevant clinical biomarkers and numerous studies investigating treatment optimization in advanced prostate cancer are reviewed in Chapter 2.

Seviteronel, a next-generation CYP17-lyase inhibitor, was proposed to overcome enzalutamide resistance via binding affinity to common mutated variants of the AR (e.g. T877A, F877L). ${ }^{12}$ The results of an open-label phase 2 study investigating the safety and efficacy of seviteronel treatment in patients with mCRPC previously treated with enzalutamide are evaluated in Chapter 3.

Dual inhibition of the AR and hypoxia-inducible factor (HIF)-1 $\alpha$ was shown to exert enhanced activity against downstream AR and HIF-1 $\alpha$ target genes, while also resensitizing AR-V7 expressing, enzalutamide-resistant cells to enzalutamide treatment. ${ }^{13}$ NLG207, a nanoparticle-drug conjugate of camptothecin (CPT), was previously shown to down-regulate HIF-1 $\alpha$ in preclinical xenograft models. ${ }^{14-17}$ Chapter 4 investigates the efficacy of dual NLG207 and enzalutamide treatment using in vivo prostate cancer models with relevant resistance mechanisms to enzalutamide (e.g. AR overexpression, AR-V7 expression).

The quantitation of CPT plasma concentrations in clinical samples following the administration of NLG207 was necessary for analyzing the pharmacokinetics (PK) observed in treated patients. Chapter 5 describes a novel, robust, selective, accurate and precise bioanalytical method to quantitate CPT concentrations, both bound and unbound 
to NLG207 nanoparticles, using liquid chromatographic separation and tandem mass spectrometric detection (UHPLC-MS/MS).

Previous NLG207 PK study utilized non-compartmental analysis to separately evaluate bound and free CPT disposition, leaving an inadequate understanding of the release of CPT from the NLG207 formulation and associated clinical implications. ${ }^{18} \mathrm{~A}$ population PK (popPK) model using data from patients with advanced solid tumors was developed in Chapter 6 to characterize the complex PK of NLG207 and better describe CPT release from nanoparticles.

NLG207 was shown to be safe, tolerable, and efficacious in several phase 2 clinical investigations spanning multiple tumor types. ${ }^{16,18-22}$ Tumor analysis from clinical studies have also demonstrated the efficacy of NLG207 against HIF-1 $\alpha$ via the down-regulation of downstream targets of angiogenesis, VEGF and CAIX..$^{23}$ Via the down-regulation of mechanisms of acquired resistance (e.g. AR-V7 activity, HIF-1 $\alpha-$ mediated transcriptional activation), NLG207 treatment was proposed to re-sensitize prostate cancer cells to enzalutamide treatment in patients with MCRPC following prior treatment with enzalutamide. An open-label phase 2 study evaluating the safety and efficacy of combination NLG207 and enzalutamide therapy in pre-treated patients with mCRPC is described in Chapter 7. An interim analysis of the current data obtained on study is provided, in addition to planned analyses with respect to clinical outcomes and relevant pharmacodynamic biomarkers of enzalutamide resistance. 


\section{References:}

1. Tran C, Ouk S, Clegg NJ, et al. Development of a second-generation antiandrogen for treatment of advanced prostate cancer. Science 2009;324:787-90. 2. Scher HI, Fizazi K, Saad F, et al. Increased survival with enzalutamide in prostate cancer after chemotherapy. N Engl J Med 2012;367:1187-97.

3. Sugawara T, Baumgart SJ, Nevedomskaya E, et al. Darolutamide is a potent androgen receptor antagonist with strong efficacy in prostate cancer models. Int $\mathbf{J}$ Cancer 2019;145:1382-94.

4. Rice MA, Malhotra SV, Stoyanova T. Second-Generation Antiandrogens: From Discovery to Standard of Care in Castration Resistant Prostate Cancer. Front Oncol 2019;9:801.

5. Sartor O, de Bono JS. Metastatic Prostate Cancer. N Engl J Med 2018;378:64557.

6. Nakazawa M, Paller C, Kyprianou N. Mechanisms of Therapeutic Resistance in Prostate Cancer. Curr Oncol Rep 2017;19:13.

7. Robinson D, Van Allen EM, Wu YM, et al. Integrative clinical genomics of advanced prostate cancer. Cell 2015;161:1215-28.

8. Annala M, Vandekerkhove G, Khalaf D, et al. Circulating Tumor DNA Genomics Correlate with Resistance to Abiraterone and Enzalutamide in Prostate Cancer. Cancer Discov 2018;8:444-57.

9. Antonarakis ES, Lu C, Luber B, et al. Androgen Receptor Splice Variant 7 and Efficacy of Taxane Chemotherapy in Patients With Metastatic Castration-Resistant Prostate Cancer. JAMA Oncol 2015;1:582-91.

10. Mateo J, Seed G, Bertan C, et al. Genomics of lethal prostate cancer at diagnosis and castration resistance. J Clin Invest 2020;130:1743-51.

11. Abida W, Armenia J, Gopalan A, et al. Prospective Genomic Profiling of Prostate Cancer Across Disease States Reveals Germline and Somatic Alterations That May Affect Clinical Decision Making. JCO Precis Oncol 2017;2017.

12. Norris JD, Ellison SJ, Baker JG, et al. Androgen receptor antagonism drives cytochrome P450 17A1 inhibitor efficacy in prostate cancer. J Clin Invest 2017; 127:2326-38.

13. Fernandez EV, Reece KM, Ley AM, et al. Dual targeting of the androgen receptor and hypoxia-inducible factor 1alpha pathways synergistically inhibits castration-resistant prostate cancer cells. Mol Pharmacol 2015;87:1006-12.

14. Lin CJ, Lin YL, Luh F, Yen Y, Chen RM. Preclinical effects of CRLX101, an investigational camptothecin-containing nanoparticle drug conjugate, on treating glioblastoma multiforme via apoptosis and antiangiogenesis. Oncotarget 2016;7:4240821.

15. Conley SJ, Baker TL, Burnett JP, et al. CRLX101, an investigational camptothecin-containing nanoparticle-drug conjugate, targets cancer stem cells and impedes resistance to antiangiogenic therapy in mouse models of breast cancer. Breast Cancer Res Treat 2015;150:559-67.

16. Pham E, Birrer MJ, Eliasof S, et al. Translational impact of nanoparticle-drug conjugate CRLX101 with or without bevacizumab in advanced ovarian cancer. Clin Cancer Res 2015;21:808-18. 
17. Pham E, Yin M, Peters CG, et al. Preclinical Efficacy of Bevacizumab with CRLX101, an Investigational Nanoparticle-Drug Conjugate, in Treatment of Metastatic Triple-Negative Breast Cancer. Cancer Res 2016;76:4493-503.

18. Weiss GJ, Chao J, Neidhart JD, et al. First-in-human phase 1/2a trial of CRLX101, a cyclodextrin-containing polymer-camptothecin nanopharmaceutical in patients with advanced solid tumor malignancies. Invest New Drugs 2013;31:986-1000. 19. Chao J, Lin J, Frankel P, et al. Pilot trial of CRLX101 in patients with advanced, chemotherapy-refractory gastroesophageal cancer. J Gastrointest Oncol 2017;8:962-9. 20. Keefe SM, Hoffman-Censits J, Cohen RB, et al. Efficacy of the nanoparticledrug conjugate CRLX101 in combination with bevacizumab in metastatic renal cell carcinoma: results of an investigator-initiated phase I-IIa clinical trial. Ann Oncol 2016;27:1579-85.

21. Sanoff HK, Moon DH, Moore DT, et al. Phase I/II trial of nano-camptothecin CRLX101 with capecitabine and radiotherapy as neoadjuvant treatment for locally advanced rectal cancer. Nanomedicine 2019;18:189-95.

22. Voss MH, Hussain A, Vogelzang N, et al. A randomized phase II trial of CRLX101 in combination with bevacizumab versus standard of care in patients with advanced renal cell carcinoma. Ann Oncol 2017;28:2754-60.

23. Gaur S, Wang Y, Kretzner L, et al. Pharmacodynamic and pharmacogenomic study of the nanoparticle conjugate of camptothecin CRLX101 for the treatment of cancer. Nanomedicine 2014;10:1477-86. 


\section{Chapter 2}

Combatting Resistance to Second-Generation Androgen Receptor Antagonists: Clinically Relevant Biomarkers and Novel Treatment Strategies in Metastatic Prostate Cancer

Keith T. Schmidt ${ }^{1}$, Alwin D. R. Huitema ${ }^{2,3}$, Cindy H. Chau ${ }^{4}$, and William D. Figg ${ }^{1,4}$

${ }^{1}$ Clinical Pharmacology Program, Center for Cancer Research, National Cancer Institute, National Institutes of Health, Bethesda, MD, USA

${ }^{2}$ Dept. Pharmacy \& Pharmacology, Netherlands Cancer Institute, Amsterdam, The Netherlands

${ }^{3}$ Department of Clinical Pharmacy, University Medical Center Utrecht, Utrecht University, Utrecht, The Netherlands

${ }^{4}$ Genitourinary Malignancies Branch, Center for Cancer Research, National Cancer Institute, National Institutes of Health, Bethesda, MD

\section{Submitted}




\section{Abstract}

The introduction of second-generation androgen receptor antagonists (SG-ARAs) has greatly impacted the standard of care treatment of metastatic prostate cancer, providing tolerable and efficacious alternatives to chemotherapy. Despite significant improvements in clinical outcomes in the setting of castration sensitivity and castration resistance, the durability of clinical response to enzalutamide, apalutamide, and darolutamide is limited by inevitable acquired resistance. Clinically relevant biomarkers of resistance to SG-ARAs have been identified in numerous studies, including alterations of the androgen receptor, DNA repair, cell cycle, PI3K/AKT, and WNT/ $\beta$ catenin pathways. To combat resistance, clinical investigators have explored approaches to optimize the utility of available treatments, while venturing into the use of alternative agents with a variety of targets, including AR-V7, AKT, EZH2, and HIF-1 $\alpha$. Ongoing research to establish predictive biomarkers for the treatment of tumors with resistance to SG-ARAs has led to the recent approvals of the PARP inhibitors, olaparib and rucaparib. Results from ongoing studies will help shape precision medicine in prostate cancer and further optimize treatment paradigms to maximize clinical outcomes. This review will discuss relevant biomarkers of acquired resistance to SG-ARA therapy and newer therapeutic approaches to further improve clinical outcomes in advanced prostate cancer. 


\section{Introduction}

Prostate cancer treatment has centered on suppressing androgen-mediated signaling since the discovery of hormonal sensitivity by Huggins and Hodges in $1941 .{ }^{1}$ Testosterone suppression, achieved via surgical orchiectomy or medical castration via administration of either a LHRH agonist or antagonist (collectively called androgen deprivation therapy, or ADT), remains the cornerstone of treatment in patients with disease progression beyond the localized setting. ${ }^{2}$ The addition of first-generation androgen receptor antagonists (FG-ARAs) (flutamide, nilutamide, and bicalutamide) to castration provided improvements in overall survival (OS) for patients with untreated prostate cancer in several clinical trials, but only showed marginal improvement in a larger meta-analysis. ${ }^{3-6}$ Further, in the setting of castration resistance, FG-ARAs did not improve survival, long supporting androgen-independence in the pre-treated metastatic setting; such tumors were only shown to be susceptible to docetaxel (DOCE), approved for the treatment of metastatic castration-resistant prostate cancer (mCRPC) in $2004 .^{7}$ Optimization of ARA medicinal chemistry resulted in the development of enzalutamide (ENZA), the first of the second-generation ARAs (SG-ARAs), which showed significant activity in patients with mCRPC. ${ }^{8,9}$ ENZA, along with abiraterone acetate (AAP), a CYP17 inhibitor administered with prednisone, provided efficacious alternatives to chemotherapy for patients with $\mathrm{mCRPC}$, while greatly influencing subsequent drug development. ${ }^{10}$

In recent years, the class of SG-ARAs has grown to include apalutamide (APA) and darolutamide (DARO), while expanding on-label indications to patients with metastatic castration-sensitive prostate cancer (mCSPC) and non-metastatic CRPC 
(nmCRPC).${ }^{11}$ Despite these successes in drug development, SG-ARAs remain susceptible to acquired resistance, both limiting durability of response and justifying further research to address an unmet clinical need. ${ }^{10,12}$ In response, drug development in prostate cancer has focused greatly on clinically relevant biomarkers of resistance and treatment strategies to limit and/or overcome potential pathways of resistance. ${ }^{12,13}$ The purpose of this review is to 1) summarize the data leading to the approval of SG-ARAs, 2) discuss clinically relevant biomarkers of resistance, and 3) outline strategies to combat acquired resistance, both currently implemented in clinical practice and under investigation.

\section{Approved Second-Generation Androgen Receptor Antagonists}

Critical to the improved clinical outcomes with SG-ARAs was a significant increase in on-target potency and disruption of AR functionality. In comparison to bicalutamide (BICA), the reported $\mathrm{IC}_{50}$ values for $\mathrm{AR}$ wild-type antagonism and disruption of the $\mathrm{N}$ and $\mathrm{C}$ terminal domain interaction (important for $\mathrm{AR}$ transcriptional activation) are at least 2.4-fold and 1.5-fold lower, respectively, for SG-ARAs. ${ }^{14}$ Further cell transactivation assays show that SG-ARAs retain antagonistic activity against numerous AR mutants (W742C/L, M896T/V), which facilitate agonistic activity in the presence of BICA. AR dimerization, a function essential for translocation into the nucleus, was reduced by greater than $47 \%$ following treatment with SG-ARAs in comparison to BICA. ${ }^{14}$ The STRIVE trial, a head-to-head comparison of ENZA to BICA in patients with CRPC (non-metastatic or metastatic), showed ENZA treatment provided a 13.7 month improvement in median progression-free survival (PFS) and 76\% reduction in risk of progression or death. ${ }^{15}$ It's worth noting that DARO has some 
advantages over ENZA and APA with respect to in vitro activity, however, the clinical evidence supporting currently approved indications, primarily nmCRPC, suggests no significant advantage at this time. ${ }^{16-18}$ With respect to acquired resistance, the effectiveness of DARO against the F877L (also defined early on in the literature as F876L) mutation will be discussed in more detail later in this review. ${ }^{14}$ Important phase III trials assessing SG-ARA treatment are summarized in Table 1.

\section{Enzalutamide (ENZA)}

ENZA was first shown to be effective in patients with mCRPC following prior treatment with DOCE, leading to its FDA approval in 2012. ${ }^{19}$ The AFFIRM study, a phase III, double-blind, placebo-controlled trial assessing ENZA in the second-line setting, showed a 5.2 month improvement in radiographic PFS (rPFS) and a significant 4.8 month improvement in OS. With the exception of an increased likelihood of seizure (accounted for in the exclusion criteria), ENZA had a minimal side effect profile, with patients only experiencing higher rates of fatigue, diarrhea, and hot flashes. AFFIRM highlighted an improvement in quality of life (QoL) scores by $25 \%$ and an increase in time to first skeletal related event (SRE) of 3.4 months in patients treated with ENZA. ${ }^{9}$ The PREVAIL study, a phase III trial assessing ENZA treatment in chemotherapy-naïve patients with mCRPC, revealed 14.6 and 4.0 month improvements in rPFS and OS, respectively. ${ }^{20} \mathrm{ENZA}$ in the first-line setting presented a similar toxicity profile, delay to first SRE, and QoL improvement, while also delaying initiation of chemotherapy by 17 months. ${ }^{21}$ Results from AFFIRM and PREVAIL established ENZA monotherapy with 
maintenance of castrate levels of testosterone as a standard of care (SOC) treatment option for mCRPC; ENZA is the only SG-ARA currently with this indication.

Results of the CHAARTED and STAMPEDE studies collectively supported the role of administering DOCE in combination with ADT in patients with mCSPC and high volume of disease. ${ }^{22,23}$ ENZA plus ADT was thus evaluated in patients with mCSPC in two separate phase III randomized controlled trials, ARCHES and ENZAMET. ${ }^{24,25}$ ENZAMET, unlike ARCHES, was an open-label study that used FG-ARA treatment as the comparator instead of placebo, while also allowing patients to receive DOCE onstudy. ENZAMET's primary endpoint was overall survival, whereas ARCHES assessed rPFS. ARCHES first showed that adding ENZA to ADT significantly reduced risk of radiographic progression or death (hazard ratio [HR], 0.39; 95\% confidence interval [CI], 0.30 to $0.50 ; \mathrm{P}<0.001)$ with minimal effect on the rate of severe adverse events. ${ }^{24}$ In the ENZAMET study, the use of ENZA over a FG-ARA with or without DOCE resulted in a $30 \%$ increase in the number of patients without PSA progression and $27 \%$ increase in patients without clinical progression after 3 years. ${ }^{25}$ Though OS data was still maturing at the time of publication, ENZAMET reported 102 deaths in the ENZA arm compared to 143 deaths in the comparator arm. Early sub-group analysis with respect to OS suggested ENZA + ADT alone provided a significant benefit to patients with low volume disease. The advantage of using ENZA over a FG-ARA when added to DOCE in the context of high or low volume disease appeared negligible. ENZAMET also reported an increased rate of DOCE-related toxicities in patients receiving both DOCE and ENZA. ${ }^{25}$ Though much is still to be learned, these studies determined ENZA treatment in patients with $\mathrm{mCSPC}$ had similar anti-tumor activity to AAP over a 3-year 
span (as reported by the STAMPEDE and LATITUDE studies) ${ }^{26-28}$ without need of coadministered corticosteroids, while also providing an equally efficacious alternative to DOCE monotherapy.

ENZA was also evaluated in patients with rapidly rising PSA but no evidence of metastatic disease after continuous ADT (i.e., nmCRPC). The FDA recently recognized metastasis-free survival (MFS) as a clinically meaningful endpoint for drug approval in nmCRPC based on impracticalities of assessing the OS endpoint and clinically relevant events associated with metastasis (e.g., pain, additional intervention), so long as the magnitude of clinical benefit was significant. ${ }^{29}$ The PROSPER study was a phase III double-blind, placebo-controlled study assessing ENZA efficacy in men with nmCRPC presenting with PSA doubling times of 10 months or less. ${ }^{18}$ In comparison to placebo, ENZA was shown to increase MFS by 21.9 months (36.6 vs. 14.7 mos; HR, 0.29; 95\% CI, 0.24 to $0.35 ; \mathrm{P}<0.001$ ) while also significantly delaying initiation to subsequent line of treatment (39.6 vs. 17.7 mos; HR, 0.25; $\mathrm{P}<0.001$ ). Of the patients receiving ENZA, $76 \%$ of the patients achieved a PSA decline of $50 \%$ or greater. Total reported adverse events and specifically Grade 3 AEs were greater in patients receiving ENZA, with $>5 \%$ increases in fatigue, hypertension, fall, dizziness, and decreased appetite irrespective of grade. ${ }^{18}$ A recent follow-up analysis of the PROSPER study has shown a 10.7 month improvement in OS associated with ENZA treatment. ${ }^{30}$

Apalutamide (APA)

APA was similarly assessed in patients with mCSPC and nmCRPC. TITAN was a phase III double-blind, randomized placebo-controlled study assessing APA plus ADT 
in patients with mCSPC irrespective of prior localized therapy or DOCE treatment. APA treatment resulted in a $20.7 \%$ and $8.9 \%$ increase in the percentage of patients with rPFS and OS, respectively, compared to placebo after 24 months. ${ }^{31}$ SPARTAN, a similar phase III study to PROSPER that also enrolled men with nmCRPC and a PSA doubling time of 10 months or less, demonstrated a significant increase in MFS of 23.3 months following APA treatment; ${ }^{17}$ a recent follow-up analysis demonstrated a significant $25 \%$ reduction in the risk of death with APA treatment, despite not reaching not reaching median OS for either arm. ${ }^{32}$ Both studies highlighted differences in the adverse event profile of APA compared to ENZA, with rash, weight loss, and hypothyroidism reported in addition to fatigue, hypertension, diarrhea and hot flush; an increase in Grade 3/4 adverse events was only reported on the SPARTAN study. ${ }^{17,31}$

\section{Darolutamide (DARO)}

ARAMIS is currently the only completed phase III clinical trial to evaluate DARO, assessing efficacy in patients with nmCRPC with PSA doubling times of 10 months or less. DARO treatment significantly improved MFS by 22 months in comparison to placebo. At time of initial publication, DARO provided a significant $29 \%$ reduction in the risk of death, ${ }^{16}$ a finding confirmed with subsequent follow-up analysis, despite not reaching median OS for either arm (HR, 0.69; $95 \% \mathrm{CI} 0.53$ to 0.88 ; $\mathrm{P}=0.003) .{ }^{33}$ Compared to other studies, DARO appeared to have a more favorable adverse event profile over ENZA and APA, with less Grade 3 events reported. Additionally, patients receiving DARO did not have significantly higher rates of fatigue, hot flush, fracture/falls, hypothyroidism, hypertension, or rash; notably, patients were 
not excluded from enrollment for prior history of seizure and only 2 patients reported a seizure while on study. ${ }^{16}$

\section{Clinical Biomarkers and Pathways of Resistance}

Tumor heterogeneity and persistent tumor phenotypes afflicting patients with high-risk or advanced prostate cancer provide several mechanisms of acquired resistance to SG-ARAs. ${ }^{34}$ Using data from the SU2C-PCF cohort, Robinson et al. first provided a framework of common alterations observed in patients with mCRPC, highlighting the AR, PI3K, WNT, cell cycle, and DNA repair pathways as targetable for precision oncology implementation. ${ }^{35}$ Abida et al. expanded upon this approach, summarizing the changes in the frequency of clinically relevant biomarkers from localized prostate cancer to $\mathrm{mCSPC}$ to $\mathrm{mCRPC}$, using data from the MSK-IMPACT molecular profiling efforts. ${ }^{36}$ With the expansion of SG-ARA approvals earlier in the disease course, the presence of such biomarkers enables potential optimization of therapy and improved clinical outcomes. A summary of the prevalent biomarkers present in patients with advanced prostate cancer is provided in Table 2.

\section{AR Pathway}

AR genomic alterations are highly prevalent in patients with mCRPC in comparison to $\mathrm{mCSPC}$, secondary to selective pressures associated with continuous ADT. ${ }^{35,36}$ These alterations are categorized via AR amplification, point mutations, or splice variation, though wide diversity of AR gene alterations is suggested in the literature ${ }^{37}$ All three mechanisms are associated with increased activation of the AR, facilitating resistance to AR-targeted therapies. Additional genes of interest associated 
with the AR pathway include SPOP, FOXA1, ZBTB16, and TMPRSS2-ERG fusions. ${ }^{35,38}$

AR amplification constituted the majority of detected AR alterations present in tumor biopsies of patients with untreated mCRPC. ${ }^{35}$ Analysis of cell-free DNA (cfDNA) collected from patients following ENZA treatment has also shown a high frequency of AR copy number gain compared to patients progressing on AAP..$^{39,40} \mathrm{AR}$ copy number gain, associated with AR amplification, has significant inter-patient variability, with a median of 7.1 AR copies (maximum of 56) reported in 65 of 115 patients with mCRPC previously treated with either AAP or ENZA (AAP/ENZA) ${ }^{41}$ Analysis of these 65 patients highlighted an increased risk of early disease progression in patients with AR amplification (HR: 2.92, 95\% CI, 1.59 to 5.37).$^{41}$ Trends towards worse PFS have been observed in patients with 8 or more AR copies. ${ }^{41}$

AR point mutations are prevalent in cf-DNA samples collected in patients with mCRPC. Common point mutations in patients with mCRPC include F877L, H874Y, T877A, and L702H, all present in the AR ligand-binding domain (LBD). ${ }^{40-42}$ ENZA and APA have been shown to have agonist activity when binding to the AR F877L mutation in vitro ${ }^{43}$ clinical detection of the F877L mutation in cf-DNA samples following SGARA treatment has been limited. ${ }^{40,44}$ DARO has higher in vitro potency to the majority of LBD containing AR-variants, with antagonist activity towards the F877L mutation. ${ }^{14,45}$ The H874Y and T877A mutations are associated with AR promiscuity, enabling estrogens and progestins to activate the AR pathway. ${ }^{46}$ Binding affinity for glucocorticoids is also possible via the $\mathrm{H} 875 \mathrm{Y}$ and $\mathrm{L} 702 \mathrm{H}$ mutations, the latter associated with AAP therapy and AR activation via prednisone..$^{47}$ Of note, activation of 
the glucocorticoid receptor (GR) pathway is considered an additional mechanism of resistance. ${ }^{48}$ The H874Y, T877A and L702H mutations have been observed in patients with mCRPC pre- and post-ENZA treatment. ${ }^{42}$

ENZA resistance has also been associated with AR-V7 expression, a constitutively active AR splice variant lacking the LBD critical for SG-ARA activity. ${ }^{49,50}$ Antonarakis et al. first detected frequent AR-V7 mRNA expression in circulating tumor cells (CTCs) in 39\% of patients with mCRPC treated with ENZA. Compared to AR-V7 negative patients, AR-V7 positive patients treated with ENZA had shorter PFS and OS. ${ }^{51}$ In addition to the mRNA-based Adna-test platform, AR-V7 can also be detected via the EPIC sciences CTC-based platform, an immunofluorescencebased detection assay that measures AR-V7 protein localized in the nucleus. ${ }^{52}$ The PROPHECY study evaluated the utility of the mRNA- and protein-based platforms, finding that both platforms were sufficient to detect AR-V7 and similarly show decreased PFS and OS following AAP/ENZA treatment. ${ }^{53}$

\section{Lineage Plasticity \& Neuroendocrine Differentiation}

In comparison to mCSPC, mCRPC is enriched for loss of the key tumor suppressors, retinoblastoma-1 (RB1) and tumor protein p53 (TP53). ${ }^{35,36} \mathrm{~A}$ recent study highlighted alterations to AR, RB1, and TP53 were statistically associated with shorter time to progression (TTP) on AAP/ENZA, with RB1 alterations associated with poor survival. ${ }^{54}$ Loss of both TP53 and RB1 can enable tumors to lose AR dependence, upregulate genes associated with cellular plasticity and pluripotency (SOX2, BRN2), and activate alternative cell signaling pathways. ${ }^{55-57}$ Epigenetic reprogramming can occur, altering DNA methylation and upregulating the histone methyltransferase, EZH2; 
EZH2 inhibition has been shown to restore sensitivity to SG-ARAs.$^{58}$ Overexpression of N-Myc and EZH2 promotes ATM upregulation, facilitating ENZA resistance via neuroendocrine differentiation..$^{58,59}$ The resulting stem-cell-like phenotype is frequently associated with neuroendocrine or small-cell lineages, enabling adaptation to selective pressure to confer ENZA resistance. ${ }^{60}$ In a study that identified 27 of 160 patients with small-cell histology, 4 of those patients previously received ENZA and 6 had received both AAP and ENZA. ${ }^{61}$ RB1 loss alone also has implications on cell cycle function; unable to prevent E2F from interacting with cyclin-dependent kinases (CDKs), RB1 loss and/or up-regulation of CDKs can enable progression to the S-Phase and promote uncontrolled cell proliferation. ${ }^{62} \mathrm{CHD} 1$ loss was also found to be associated with chromatin dysregulation and lineage plasticity, resulting in heterogeneous mechanisms of ENZA resistance, including tumors driven by the GR, TBX2, BRN2, or NR2F1.63 Tumors displaying neuroendocrine phenotypes, often categorized by synaptophysin, chromogranin A, and/or neuro-specific enolase expression, are typically treated with platinum-based regimens. ${ }^{64}$

\section{DNA Repair}

Defects in DNA repair, notably homologous recombination repair (HRR), confer both prognostic and predictive implications in prostate cancer treatment. ${ }^{35,36,65}$ The most notable HRR genes include BRCA1 and BRCA2, with indirect contribution of ATM, a DNA-damage checkpoint that activates HRR. Additional less established genes of interest include CHEK2, BRIP1, RAD51D, PALB2 and FANCL. ${ }^{13}$ Patients with metastatic prostate cancer and/or familial history of oncologic disorders, notably ovarian and breast cancer, are encouraged to undergo germline testing, per the National 
Comprehensive Cancer Network (NCCN) recommendations. ${ }^{66}$ Defects of the HRR pathways have demonstrated sensitivity to poly(ADP-ribose) polymerase (PARP) inhibition via synthetic lethality, providing clinically meaningful outcomes in patients previously treated with ENZA. ${ }^{13,67}$ Such tumors harboring HRR mutations are also sensitive to platinum agents. ${ }^{68-70}$

While prostate cancer is deemed an "immunologically cold" histology, DNA repair defects have indicated a potential role of PD-1/PD-L1 targeted immunotherapy, especially in patients who have received numerous lines of therapy. ${ }^{71}$ Germline and somatic mutations in mismatch repair (dMMR) genes (e.g., MSH2, MLH1) can enable hypermutation and microsatellite instability (MSI) in tumors. ${ }^{72}$ Patients with dMMR mutations and/or MSI-high tumors have increased immune filtration and upregulation of tumor neo-antigens, including PD-L1. ${ }^{73}$ These findings led to the approval of pembrolizumab, an anti-PD-1 targeted agent, for patients with dMMR or MSI-high tumors of any histology once all SOC treatments are exhausted. ${ }^{74}$ More recently, the FDA approved pembrolizumab for patients with solid tumors and high mutational burden (TMB-H; $\geq 10$ mutations/megabase $[\mathrm{mut} / \mathrm{Mb}]$ ) based on currently unpublished data from the KEYNOTE-158 study (NCT02628067). ${ }^{75}$ In a cohort of 1033 patients with prostate cancer and adequate tumor tissue available, 23 patients had positive MSIsensor scores (a quantitative measure of MSI status), of which 7 had a pathogenic germline dMMR mutation. Of eleven patients with dMMR/MSI-high CRPC receiving PD-1/PD-L1 therapy, 6 patients had significant PSA declines, with 4 showing radiographic response. ${ }^{72}$ Mutations that inactivate CDK12, typically enriched following 
progression on ADT, are also associated with shorter TTP on ENZA; $;^{36,76}$ such mutations have been suggested to predict response to PD-1/PD-L1 targeted therapy. ${ }^{77,78}$

\section{PI3K/AKT/mTOR Pathway}

Loss of PTEN, a key tumor suppressor moderating the PI3K/AKT pathway, is more prevalent in patients with $\mathrm{mCRPC}$ versus $\mathrm{mCSPC}$ and is associated with ENZA resistance. ${ }^{35,36,54}$ In 144 patients treated with AAP, PTEN loss correlated with shorter treatment duration and OS. ${ }^{79}$ Preclinical studies have shown significant tumor growth inhibition via co-targeting both the PI3K and AR pathways. ${ }^{80,81}$ ENZA treatment has been previously shown to activate PI3K/AKT signaling via the stabilization of the AKT phosphatase; PTEN loss reduces regulation of PI3K/AKT signaling, further enabling AKT activation via cross-regulation of both AR and PI3K pathways by reciprocal feedback..$^{82,83}$ The PI3K pathway could also be enhanced by amplification or activating mutations (e.g., PIK3CB, AKT1), though such alterations occur less frequently. ${ }^{35}$ Lastly, assessment of CTCs in 16 patients with mCRPC showed frequent genomic alterations in $\mathrm{AR}, \mathrm{PI} 3 \mathrm{~K}$ and c-MET signaling $;{ }^{84} \mathrm{c}-\mathrm{MET}$ has promiscuous functionality in cancer, with potential to activate the PI3K/AKT/mTOR, Ras/MAPK, JAK/STAT, and Wnt/ $\beta$-catenin pathways. ${ }^{85}$

\section{Wnt/ $\beta$-catenin Pathway}

Clinically relevant mutations stimulating Wnt/ $\beta$-catenin pathway signaling have been identified in CTNNB1, APC, and RNF43, ${ }^{86}$ with near equivalent representation in patients with mCSPC and mCRPC. ${ }^{36}$ Mutations that activate CTNNB1 or inactivate either APC or RNF43 were found in 11\% of patients with mCRPC progressing on first- 
line AAP/ENZA; patients with Wnt-activating mutations had shorter time to PSA progression and $\mathrm{OS} .{ }^{87}$ In 101 patients with $\mathrm{MCRPC}, \beta$-catenin mutations were shown to be exclusive to patients with ENZA-resistance and an independent predictor of worse OS. ${ }^{88}$

$\beta$-catenin signaling is also relevant in hypoxic tumors. Intratumoral hypoxia is a noted feature of aggressive prostate cancers promoting the up-regulation of HypoxiaInducible Factor (HIF)-1 $\alpha$, a transcription factor responsible for tumor angiogenesis, anaerobic metabolism, immunity, adaptation and metastatic potential. ${ }^{89-93} \mathrm{~A}$ ternary complex on androgen response elements comprising of HIF-1 $\alpha$, AR, and $\beta$-catenin was suggested to facilitate cross-talk between the HIF and AR pathways. ${ }^{94,95}$ Evidence of such cross-talk has been demonstrated in vitro via dual inhibition of HIF-1 $\alpha$ and AR, highlighting synergistic down-regulation of AR- and HIF-mediated transcription, VEGF expression, and cell proliferation..$^{96}$

\section{Approaches to Overcome SG-ARA Acquired Resistance Using Available}

\section{$\underline{\text { Treatments }}$}

With numerous anti-cancer agents to treat patients with mCRPC, investigators have sought to optimize treatment selection and maximize the effectiveness of each line of therapy. ${ }^{97}$ Approaches to improve clinical outcomes and combat acquired resistance to SG-ARA treatment focus on treatment sequence, earlier introduction of SG-ARAs, implementation of precision medicine and evaluation of combination regimens. Treatment optimization has emphasized appropriate treatment goals and clinical trial 
endpoints, defined by the prostate cancer working group (PCWG) ${ }^{98}$ Important trials with currently approved agents are summarized in Table 3.

\section{Optimizing Treatment Sequence}

ENZA, AAP, and DOCE are equally efficacious first-line treatment options for mCRPC,${ }^{99}$ however, approaches to limit acquired resistance and maximize OS are unclear. Caffo et al. evaluated treatment sequence permutations of ENZA, AAP, or cabazitaxel up to fourth-line treatment in patients with mCRPC who previously received first-line DOCE. ${ }^{100}$ In a retrospective cohort of 260 patients, similar PSA response, PFS and OS were reported for second- and third-line treatment regardless of agent used. ${ }^{100}$ The CARD study used a randomized, open-label design to compare cabazitaxel with $\mathrm{AAP} / \mathrm{ENZA}$ in patients with $\mathrm{mCRPC}$ who previously received DOCE and AAP/ENZA (AAP-experienced patients received ENZA, vice versa). Patients receiving cabazitaxel had a small but significant benefit with respect to PFS (4.4 vs. 2.7 months; HR, 0.52; 95\% CI, 0.40 to $0.68 ; \mathrm{P}<0.001)$ and $\mathrm{OS}(13.6$ vs. 11.0 months; HR, $0.64 ; 95 \% \mathrm{CI}, 0.46$ to $0.89 ; \mathrm{P}=0.008)(\mathrm{NCT} 02485691) .{ }^{101}$ Another randomized open-label phase II study showed sequencing AAP then ENZA lengthened time to second PSA progression in comparison to ENZA then AAP without increased OS (NCT02125357). ${ }^{102}$ An ongoing phase III study is evaluating second-line DOCE or hormonal therapy in patients with asymptomatic or oligometastatic mCRPC following AAP/ENZA treatment (NCT04139772). These studies suggest accumulated acquired resistance with successive lines of therapies diminishes clinical improvement. Alternating between antihormonal therapy and chemotherapy with successive lines of therapy is generally accepted as a SOC treatment approach in mCRPC. ${ }^{103}$ Incorporation of either sipuleucel- 
$\mathrm{T}$ or radium-223 in studies evaluating sequence-related clinical outcomes is currently lacking.

\section{Investigational Indications and Combinations}

Ongoing phase III studies are exploring indications earlier in the prostate cancer disease course. The addition of APA in patients with a recently detectable PSA following radiation and ADT for 6-8 months is currently being investigated. (NCT03777982). Stereotactic body radiotherapy (SBRT) in combination with ENZA and ADT to treat oligometastases is also being investigated (NCT02685397). Additionally, numerous studies are evaluating SG-ARA therapy in localized prostate cancer, including combination treatment with radiation (NCT04136353, NCT03488810, NCT02531516, NCT02446444), neoadjuvant treatment prior to radical prostatectomy (NCT03860987), and salvage therapy post-radical prostatectomy (NCT04134260).

Combination approaches using SG-ARA therapy are also being investigated. The Alliance A031201 study evaluated ENZA and AAP vs. ENZA alone in patients with treatment-naïve $\mathrm{mCRPC}$, demonstrating no significant OS benefit and increased toxicity with combination therapy (NCT01949337). ${ }^{104}$ A similar study investigating APA plus AAP is currently ongoing (NCT02257736), with recent phase Ib data suggesting antitumor activity and no potential drug-drug interaction. ${ }^{105}$ Two phase III studies are evaluating AAP in combination with SG-ARA therapy; STAMPEDE (NCT00268476) and the "Androgen Annihilation" study (NCT03009981) are investigating the addition of ENZA in mCSPC and APA in nmCRPC, respectively. A phase III study adding DARO to SOC DOCE in mCSPC is currently ongoing (NCT02799602). SG-ARA trials have also sought to implement radium-223 in combination treatments. Despite early 
termination of the ERA-223 study, which uncovered a high risk of fracture or death associated with AAP + RA-223 + ADT treatment in patients with mCRPC, the addition of bone-protecting agents (e.g., denosumab) and removal of prednisone could potentially enable safe administration of SG-ARAs with Radium-223. ${ }^{106-108}$ Phase III studies evaluating radium-223 in combination with SG-ARAs include PEACE-III (ENZA, NCT02194842), and ESCALATE (ENZA or DARO, NCT04237584).

\section{Precision Medicine and Biomarker-Driven Approaches}

The histology-agnostic approval of pembrolizumab for heavily pre-treated patients with MSI-high or TMB-high tumors provides a treatment option for mCRPC. Evidence of pembrolizumab efficacy is limited in patients with prostate cancer, and the approved approaches for patient enrichment appear critical for treatment success. ${ }^{72}$ The IMbassador250 study evaluated atezolizumab with ENZA in patients with mCRPC following treatment with an SG-ARA or AAP regardless of MSI status; the combination had significant toxicity and did not improve OS (NCT03016312). ${ }^{109}$ Phase III studies are currently evaluating the addition of pembrolizumab to ENZA irrespective of biomarkers in mCSPC (NCT04191096) and treatment-naïve mCRPC (NCT03834493). Further evaluation of biomarkers associated with response to PD-1/PD-L1 inhibitors is needed in patients with SG-ARA resistant prostate cancer.

The success of biomarker-driven PARP inhibitor treatment in patients with mCRPC following treatment with AAP/ENZA has changed SOC. The randomized, open-label phase II TOPARP-A study established the potential role of HRR mutations to predict response to olaparib in patients with mCRPC treated previously with taxane and AR-targeted therapy, showing associations of BRCA2 and ATM alterations with rPFS 
and OS. ${ }^{110}$ Another phase II study, TOPARP-B, confirmed significant PSA response and PFS improvement following olaparib treatment in pre-treated patients with mCRPC and BRCA1/2 mutations, while suggesting responses in patients with ATM, PALB2, FANCA, or CHEK2 aberrations. ${ }^{67}$ The PROfound study was a randomized, open-label phase III study comparing olaparib to physician's choice of hormonal agent in patients with mCRPC previously treated with a taxane and hormonal agent $(62 \%$ of patients previously received ENZA). ${ }^{13}$ Among patients with at least one alteration in BRCA1, BRCA2, or ATM, imaging-based PFS was significantly improved in those receiving olaparib (7.4 to 3.6 mos; HR, 0.34 ; $95 \%$ CI, 0.25 to 0.47 ; $<<0.001)$; median OS differed (18.5 vs. 15.1 months) though not statistically significant, with $81 \%$ of patients with progression crossing over to receive olaparib. Analysis of all 15 DNA repair aberrations included in the PROfound study yielded similar rPFS improvement with olaparib treatment. ${ }^{13}$ Another PARP inhibitor, rucaparib, was recently granted accelerated approval by the FDA based on significant objective response rate (ORR) and duration of response (DOR) seen on the TRITON2 phase II study in BRCA-mutated patients with mCRPC previously treated with a taxane and hormonal agent (NCT02952534). ${ }^{111}$ TRITON3, a confirmatory phase III study evaluating rucaparib is currently ongoing (NCT02975934). Rucaparib is only approved in patients with BRCA-mutations who received a taxane and AAP/ENZA, whereas olaparib is approved for many DNA repair mutations following AAP/ENZA without prior chemotherapy. ${ }^{13,111}$

Further potential use of precision medicine exists with CTC enumeration and AR analysis. Enumeration of CTCs was retrospectively assessed as a biomarker of treatment response in patients progressing on AAP on the COU-AA-301 study, showing improved 
OS in patients with CTC counts of $\geq 5$ cells $/ 7.5 \mathrm{~mL}$ at baseline and $30 \%$ CTC decline. ${ }^{112}$ Another assessment of treatment response includes AR-V7 detection in CTCs ${ }^{113}$ or ARFL and AR-V7 ctDNA analysis ${ }^{114}$ to determine if AR-V7+ patients are more optimally treated with a taxane over AAP/ENZA. Though several studies have suggested AR-V7 status could enhance clinical decision making, ${ }^{113,115}$ further studies (e.g., VARIANT or ISRCTN10246848) are needed to confirm predictive utility. ${ }^{116}$

The phase III ProBio study is currently evaluating the use of biomarkers to determine treatment sequence in mCRPC. The study will assign one of six treatments following pre-enrollment biomarker signature analysis, treat until disease progression, then select subsequent treatment based on an updated biomarker signature (NCT03903835).

\section{Adaptive Therapy}

The understanding of evolutionary tumor dynamics in treatment response have challenged conventional treatment goals. PCWG3 guidelines emphasize maximizing the utility of an agent until confirmed radiographic progression, ${ }^{98}$ a notion supported by a recent study demonstrating the timing discordance between rising PSA and eventual radiographic progression. ${ }^{117}$ Adaptive therapy incorporates mathematical models to predict the growth of drug-sensitive and drug-resistant subclones present in tumors in a patient-specific manner; the goal is to cycle drug selection using real-time data to prevent over-population of aggressive tumor cell phenotypes and lengthen TTP. ${ }^{118}$ Using preclinical and clinical data associated with mCRPC tumors, a potential to improve outcomes using the evolution-based strategy was first assessed with AAP monotherapy via cycling treatment on and off, ${ }^{119}$ then cycling of AAP and DOCE 
treatment. ${ }^{120}$ In a pilot study assessing adaptive AAP monotherapy, 10 of 11 patients with mCRPC maintained stable oscillations of tumor burden, with a TTP of at least 27 months and a $47 \%$ reduction in cumulative drug use compared to standard dosing (NCT02415621). ${ }^{119}$ A follow-up adaptive AAP therapy trial in patients with mCSPC is currently ongoing (NCT03511196). Computational simulations analyzing the strategic application of DOCE during AAP monotherapy demonstrated a potential significant increase in TTP. ${ }^{120}$ The push towards patient-specific multi-drug adaptive therapy requires the understanding of several factors: the frequency-dependent cycles of tumor evolution, the appropriate timing and selection of treatment, and the velocity of tumor evolution. ${ }^{118}$ It is possible the adaptive therapeutic approach could soon incorporate the use of SG-ARA therapy.

\section{$\underline{\text { Novel Investigational Therapies to Combat SG-ARA Acquired Resistance }}$}

Many investigational therapeutics and treatment regimens have been developed to address the multitude of resistance mechanisms. In addition to the therapeutics covered in this section, histology-agnostic biomarker-driven studies (or basket trials) can enroll patients with prostate cancer and relevant genetic mutations that may confer sensitivity to pre-specified treatments. ${ }^{121}$ Additionally, a Canadian "umbrella" study is prospectively screening for biomarkers in prostate cancer before selecting one of six targeted treatments (NCT03385655). Clinical trials of investigational agents relevant to SG-ARA acquired resistance are summarized in Table 4. 
The development of newer AR-targeted therapies has focused on overcoming established SG-ARA resistance mechanisms, but has frequently resulted in negative trials. The ARMOR3-SV trial attempted to investigate galeterone, an oral agent proposed to inhibit CYP17 lyase, degrade AR, and antagonize $\mathrm{AR},{ }^{122}$ in patients with AR-V7+ chemotherapy-naïve mCRPC. The study overlooked the limited presence of AR-V7 in newly diagnosed mCRPC and was closed early once deemed unlikely to meet its primary endpoint (NCT02438007). ${ }^{123}$ EPI-506, an agent targeting the N-terminal domain of the AR to combat AR-V7, was associated with a significant pill burden and failed to improve PSA responses in patients with mCRPC (NCT02606123). ${ }^{124}$ Similarly, niclosamide, an agent proposed to degrade AR-V7, was unable to provide sufficient plasma concentrations needed for anti-tumor activity (NCT02532114). ${ }^{125}$ Seviteronel, a selective CYP17 lyase inhibitor with activity against the T788A and F877L AR mutations, showed limited anti-tumor activity and clinically significant CNS-related toxicities in patients with mCRPC previously treated with ENZA (NCT02130700). ${ }^{126}$

Newer approaches to differentially target the AR pathway to overcome acquired resistance are under investigation. ARRx is an antisense oligonucleotide designed to hybridize AR mRNA to inhibit AR-FL and AR-V7 expression, ${ }^{127}$ and is currently being evaluated in combination with ENZA in patients with treatment-naïve mCRPC (NCT03300505). ARV-110 utilizes a technology called PROteolysis TArgeting Chimera (PROTAC) to promote ubiquitination and degradation of the AR. Following successful evaluation in ENZA-resistant preclinical models, ARV-110 is currently being investigated in a phase I clinical trial (NCT03888612). ${ }^{128}$ Lastly, radioligand therapies 
targeting PSMA expressing cells, such as ${ }^{177}$ Lu-PSMA-617, have shown clinically meaningful activity in patients with mCRPC previously treated with AAP/ENZA; ${ }^{129}$ NCT03042468 is one of several studies evaluating PSMA-targeted radiotherapy, enrolling previously treated patients with mCRPC.

\section{Alternative Hormonal Approaches}

Numerous clinical trials are investigating hormonal mechanisms of acquired resistance without targeting the $\mathrm{AR}$ and/or androgen biosynthesis. Bipolar androgen therapy (BAT) has been proposed to induce anti-tumor activity via rapid cycling between high and low serum testosterone concentrations in patients on continuous SGARA treatment. ${ }^{130}$ In a recent study in patients with asymptomatic mCRPC following ENZA treatment, 9 of 30 patients achieved a PSA declined of greater than $50 \%$ (PSA ${ }_{50}$ ) on BAT and 15 of 29 patients who finished a course of BAT achieved a PSA50 upon rechallenging ENZA (NCT02090114). ${ }^{130}$ Using a similar population, the TRANSFORMER study also demonstrated a 37.3 month OS following sequential BAT and ENZA, while OS was 28.6 months in patients receiving ENZA alone (NCT02286921). ${ }^{131}$ In addition to paradoxical stimulation of the AR, treatments are also targeting GR bypass. CORT125281, a GR antagonist, is currently being evaluated in combination with ENZA in patients with SG-ARA- or AAP-resistant mCRPC (NCT03437941).

\section{Epigenetics and Lineage Plasticity}

Investigational treatment has frequently targeted epigenetic regulation. To target lineage plasticity and neuroendocrine tumors, alisertib, an Aurora Kinase A inhibitor, 
was proposed to disrupt $\mathrm{N}$-myc signaling and restore AR signaling. In patients with mCRPC and evidence of neuroendocrine disease receiving alisertib, only a small subset of patients achieved a meaningful clinical response (NCT01799278). ${ }^{132}$ Bromodomain and Extraterminal (BET) chromatin reader inhibitors in combination with either ENZA or APA were shown to overcome ENZA resistance via down-regulation of AR-V7 expression and AR signaling in preclinical models, providing rationale for clinical development. ${ }^{133,134}$ A phase I expansion cohort evaluating mivebresib, a pan-BET inhibitor, suggested modest clinical activity in patients with advanced prostate cancer (NCT02391480); ${ }^{135}$ another BET inhibitor, ZEN003694, in combination with ENZA in patients with mCRPC is currently being investigated (NCT02711956). The EZH2 inhibitor, CPI-1205, is currently being evaluated for safety in combination with either AAP or ENZA in patients with mCRPC (NCT03480646).

\section{PI3K/AKT/mTOR Pathway Inhibition}

Studies that focus on PTEN loss and targeting the PI3K/AKT/mTOR pathway are currently ongoing. A recent phase II study in patients with mCRPC following taxane and AR-targeted treatment evaluated the AKT inhibitor, ipatasertib, in combination with AAP (NCT01485861). When stratified for PTEN loss, adding low dose ipatasertib (200 mg) improved rPFS by 6.5 months (11.1 vs. 4.6 mos; HR, 0.46 ; $95 \%$ CI, 0.25 to 0.83). ${ }^{136}$ Capivasertib, another AKT inhibitor, in combination with ENZA had activity in patients with mCRPC post-AAP/ENZA and either PTEN loss or AKT activating mutations (NCT02525068). ${ }^{137}$ MLN0128, a dual mTOR inhibitor, had limited success in 9 patients with mCRPC previously treated with AAP/ENZA (NCT02091531). ${ }^{138}$ Other PI3K/AKT/mTOR pathway inhibitors are being evaluated in combination with 
ENZA in patients with mCRPC, including LY3023414, GSK2636771, and everolimus (NCT02407054, NCT02215096, NCT02125084).

Angiogenesis, DNA Repair, and Immunotherapy

No validated biomarkers have been established to assess angiogenic activity in prostate cancer; however, previous studies have interrogated the utility of antiangiogenic treatment in CRPC without SG-ARA resistance. ${ }^{139-141}$ More recently, cabozantinib, a dual c-MET and VEGFR-2 inhibitor, was evaluated as monotherapy in patients with pre-treated (DOCE + AAP/ENZA) mCRPC on two separate phase III trials with prednisone or mitoxantrone as the comparator (COMET-1 and COMET-2, respectively). Though neither study yielded a significant OS benefit, a retrospective analysis combining both cohorts suggest patient enrichment based on high-risk disease and/or molecular features may select patients deriving benefit from cabozantinib monotherapy. ${ }^{142}$ Combining cabozantinib with atezolizumab was shown to be tolerable and have clinically meaningful activity in a phase $1 \mathrm{~b}$ study enrolling patients with mCRPC previously given ENZA/AAP (NCT03170960). ${ }^{143}$

Currently, the National Cancer Institute (NCI) has several phase II clinical trials treating patients with mCRPC following prior ENZA and/or AAP therapy. Two studies are investigating the use of NLG207, a nanoparticle-drug conjugate of the potent topoisomerase I inhibitor, camptothecin. ${ }^{144}$ NCT03531827 is investigating NLG207mediated inhibition of HIF- $1 \alpha$ accumulation in combination with ENZA to overcome AR and hypoxia-mediated resistance. NLG207 is also being investigated as a DNA damaging agent in combination with olaparib irrespective of DNA repair mutation status (NCT02769962). NCT02484404 is currently evaluating the efficacy of the PD-L1 
inhibitor, durvalumab, in combination with olaparib, and has already demonstrated significant rPFS, particularly in men with DNA damage repair alterations. ${ }^{145}$ Of note, KEYLYNK-010 is a phase III study currently investigating pembrolizumab and olaparib versus either ENZA or AAP in patients with mCRPC following disease progression on DOCE and AAP/ENZA (NCT03834519).

\section{$\underline{\text { Future Directions }}$}

The past decade has seen exciting research and breakthroughs for patients with high-risk and advanced prostate cancer. With continued clinical investigations, several important questions associated with SG-ARA therapy need to be addressed. Clinical decisions of SG-ARA selection are of particular interest, especially within the context of acquired resistance. Unlike ENZA and APA, DARO has activity against the F877L and T877A AR mutations, suggesting that the prevalence of these mutations may be lessened following DARO treatment. ${ }^{14}$ Enhanced AR potency and activity, in addition to a more favorable side effect profile, ${ }^{14,16-18}$ may result in increased use of DARO over ENZA or APA in patients with nmCRPC.

Initiating SG-ARA therapy earlier in the treatment armamentarium with or without the intensification of other agents can affect acquired resistance in later lines of therapy. It remains to be determined whether patterns of resistance to SG-ARA treatment earlier in the disease setting differ in comparison mCRPC; current analyses have only established patterns of biomarker enrichment from treatment naïve mCSPC to mCRPC, noting the critical differences between these stages of disease. ${ }^{36}$ The majority of data outlining acquired resistance to SG-ARA treatment is associated with ENZA, ${ }^{39}$ 
thus determining possible differences in resistance patterns across the drug class are important. Further, selecting appropriate patients for treatment intensification on the basis of poor prognostic features is also necessary. Via sub-group analysis, ENZAMET study has suggested DOCE to have similar OS in patients with mCSPC when in combination with either ENZA or a FG-ARA, indicating the use of more potent SGARAs was not necessary. ${ }^{25}$ Limiting the number of patients receiving more rigorous treatment regimens may promote increased tolerability and reduce the development of more aggressive tumor phenotypes. ${ }^{118}$ More research is needed to determine if earlier intensification of therapy is beneficial for all patients and the associated consequences for later lines of therapy are reasonable.

The importance of tumor heterogeneity and biomarker identification has led to numerous potential drug targets, including the first of hopefully many approved therapies to implement precision medicine specifically in prostate cancer (i.e. olaparib, rucaparib). With more sophisticated methods of characterizing tumors, it becomes essential to identify mechanisms underlying critical biomarker alterations. A recent preclinical report has uncovered significant heterogeneity in the mechanisms governing AR re-arrangement and amplifications,${ }^{37}$ indicating complexity beyond current ARfocused biomarker signatures. Genomic studies have shown that besides tumor histology and treatment history, mutations drive disease biology and treatment selection. Following the FDA approvals of olaparib and rucaparib, it should become routine practice for patients to undergo genomic testing following discontinuation of SG-ARA or AAP therapy to determine if follow-up PARP inhibitor therapy would be appropriate. With expanded genomic and transcriptomic profiling to better characterize alterations of 
the AR and other pathways, more optimized therapy could be selected for patients, while answering important research questions, such as the underlying mechanisms limiting taxane efficacy in some AR-V7+ patients. ${ }^{115}$ Further, improved tracking of tumor evolutionary dynamics following lines of therapy may aid in clinical decision making and promote new treatment approaches, such as adaptive therapy. ${ }^{118}$ As the repertoire of therapeutic options with predictive biomarkers continues to expand, so too will the role of precision medicine to manage acquired resistance to SG-ARA therapy.

Not unlike the tumors we aim to treat, our drug development efforts continue to evolve to meet the demands of a diverse patient population. Though many lessons about acquired resistance to SG-ARA therapy are to be learned from the plethora of clinical trials completed and ongoing, it is remarkable to remember SG-ARA therapy did not have an indication in advanced prostate cancer ten years ago. It is with continued diligence and persistence that our clinical decision-making surrounding SG-ARA therapy will be further optimized and refined in the future.

Acknowledgment: This study was supported by the Intramural Research Program of the National Institutes of Health, National Cancer Institute, Bethesda, MD, US, (ZIA BC 010453). The content of this publication does not necessarily reflect the views or policies of the Department of Health and Human Services, nor does mention of trade names, commercial products, or organization imply endorsement by the U.S. Government. 


\section{REFERENCES}

1. Huggins C, Hodges CV. Studies on prostatic cancer. I. The effect of castration, of estrogen and androgen injection on serum phosphatases in metastatic carcinoma of the prostate. CA Cancer J Clin 1972;22:232-40.

2. Gomella LG. Effective testosterone suppression for prostate cancer: is there a best castration therapy? Rev Urol 2009;11:52-60.

3. Janknegt RA. Total androgen blockade with the use of orchiectomy and nilutamide (Anandron) or placebo as treatment of metastatic prostate cancer. Anandron International Study Group. Cancer 1993;72:3874-7.

4. Crawford ED, Eisenberger MA, McLeod DG, et al. A controlled trial of leuprolide with and without flutamide in prostatic carcinoma. N Engl J Med 1989;321:419-24.

5. Kolvenbag GJ, Blackledge GR, Gotting-Smith K. Bicalutamide (Casodex) in the treatment of prostate cancer: history of clinical development. Prostate 1998;34:61-72.

6. Maximum androgen blockade in advanced prostate cancer: an overview of the randomised trials. Prostate Cancer Trialists' Collaborative Group. Lancet 2000;355:1491-8.

7. Rawlinson A, Mohammed A, Miller M, Kunkler R. The role of enzalutamide in the treatment of castration-resistant prostate cancer. Future Oncol 2012;8:1073-81.

8. Tran C, Ouk S, Clegg NJ, et al. Development of a second-generation antiandrogen for treatment of advanced prostate cancer. Science 2009;324:787-90. 9. Scher HI, Fizazi K, Saad F, et al. Increased survival with enzalutamide in prostate cancer after chemotherapy. N Engl J Med 2012;367:1187-97.

10. Sartor O, de Bono JS. Metastatic Prostate Cancer. N Engl J Med 2018;378:64557.

11. Rice MA, Malhotra SV, Stoyanova T. Second-Generation Antiandrogens: From Discovery to Standard of Care in Castration Resistant Prostate Cancer. Front Oncol 2019;9:801.

12. Nakazawa M, Paller C, Kyprianou N. Mechanisms of Therapeutic Resistance in Prostate Cancer. Curr Oncol Rep 2017;19:13.

13. de Bono J, Mateo J, Fizazi K, et al. Olaparib for Metastatic Castration-Resistant Prostate Cancer. N Engl J Med 2020.

14. Sugawara T, Baumgart SJ, Nevedomskaya E, et al. Darolutamide is a potent androgen receptor antagonist with strong efficacy in prostate cancer models. Int $\mathrm{J}$ Cancer 2019; 145:1382-94.

15. Penson DF, Armstrong AJ, Concepcion R, et al. Enzalutamide Versus Bicalutamide in Castration-Resistant Prostate Cancer: The STRIVE Trial. J Clin Oncol 2016;34:2098-106.

16. Fizazi K, Shore N, Tammela TL, et al. Darolutamide in Nonmetastatic, Castration-Resistant Prostate Cancer. N Engl J Med 2019;380:1235-46.

17. Smith MR, Saad F, Chowdhury S, et al. Apalutamide Treatment and Metastasisfree Survival in Prostate Cancer. N Engl J Med 2018;378:1408-18.

18. Hussain M, Fizazi K, Saad F, et al. Enzalutamide in Men with Nonmetastatic, Castration-Resistant Prostate Cancer. N Engl J Med 2018;378:2465-74. 
19. Ning YM, Brave M, Maher VE, et al. U.S. Food and Drug Administration Approval Summary: Enzalutamide for the Treatment of Patients With ChemotherapyNaive Metastatic Castration-Resistant Prostate Cancer. Oncologist 2015;20:960-6. 20. Beer TM, Armstrong AJ, Rathkopf D, et al. Enzalutamide in Men with Chemotherapy-naive Metastatic Castration-resistant Prostate Cancer: Extended Analysis of the Phase 3 PREVAIL Study. Eur Urol 2017;71:151-4.

21. Beer TM, Armstrong AJ, Rathkopf DE, et al. Enzalutamide in metastatic prostate cancer before chemotherapy. N Engl J Med 2014;371:424-33.

22. Sweeney CJ, Chen YH, Carducci M, et al. Chemohormonal Therapy in Metastatic Hormone-Sensitive Prostate Cancer. N Engl J Med 2015;373:737-46.

23. James ND, Sydes MR, Clarke NW, et al. Addition of docetaxel, zoledronic acid, or both to first-line long-term hormone therapy in prostate cancer (STAMPEDE): survival results from an adaptive, multiarm, multistage, platform randomised controlled trial. Lancet 2016;387:1163-77.

24. Armstrong AJ, Szmulewitz RZ, Petrylak DP, et al. ARCHES: A Randomized, Phase III Study of Androgen Deprivation Therapy With Enzalutamide or Placebo in Men With Metastatic Hormone-Sensitive Prostate Cancer. J Clin Oncol 2019;37:297486.

25. Davis ID, Martin AJ, Stockler MR, et al. Enzalutamide with Standard First-Line Therapy in Metastatic Prostate Cancer. N Engl J Med 2019;381:121-31.

26. James ND, de Bono JS, Spears MR, et al. Abiraterone for Prostate Cancer Not Previously Treated with Hormone Therapy. N Engl J Med 2017;377:338-51.

27. Fizazi K, Tran N, Fein L, et al. Abiraterone plus Prednisone in Metastatic, Castration-Sensitive Prostate Cancer. N Engl J Med 2017;377:352-60.

28. Fizazi K, Tran N, Fein L, et al. Abiraterone acetate plus prednisone in patients with newly diagnosed high-risk metastatic castration-sensitive prostate cancer (LATITUDE): final overall survival analysis of a randomised, double-blind, phase 3 trial. Lancet Oncol 2019;20:686-700.

29. Beaver JA, Kluetz PG, Pazdur R. Metastasis-free Survival - A New End Point in Prostate Cancer Trials. N Engl J Med 2018;378:2458-60.

30. Sternberg CN, Fizazi K, Saad F, et al. Enzalutamide and Survival in Nonmetastatic, Castration-Resistant Prostate Cancer. N Engl J Med 2020;382:2197-206. 31. Chi KN, Agarwal N, Bjartell A, et al. Apalutamide for Metastatic, CastrationSensitive Prostate Cancer. N Engl J Med 2019;381:13-24.

32. Small EJ, Saad F, Chowdhury S, et al. Apalutamide and overall survival in nonmetastatic castration-resistant prostate cancer. Ann Oncol 2019;30:1813-20.

33. Fizazi K, Shore N, Tammela TL, et al. Overall survival (OS) results of phase III ARAMIS study of darolutamide (DARO) added to androgen deprivation therapy (ADT) for nonmetastatic castration-resistant prostate cancer (nmCRPC). J Clin Oncol 2020;38:abstr 5514.

34. Mateo J, Seed G, Bertan C, et al. Genomics of lethal prostate cancer at diagnosis and castration resistance. J Clin Invest 2020;130:1743-51.

35. Robinson D, Van Allen EM, Wu YM, et al. Integrative clinical genomics of advanced prostate cancer. Cell 2015;161:1215-28. 
36. Abida W, Armenia J, Gopalan A, et al. Prospective Genomic Profiling of Prostate Cancer Across Disease States Reveals Germline and Somatic Alterations That May Affect Clinical Decision Making. JCO Precis Oncol 2017;2017.

37. Li Y, Yang R, Henzler CM, et al. Diverse AR Gene Rearrangements Mediate Resistance to Androgen Receptor Inhibitors in Metastatic Prostate Cancer. Clin Cancer Res 2020.

38. Wang Z, Wang Y, Zhang J, et al. Significance of the TMPRSS2:ERG gene fusion in prostate cancer. Mol Med Rep 2017;16:5450-8.

39. Torquato S, Pallavajjala A, Goldstein A, et al. Genetic Alterations Detected in Cell-Free DNA Are Associated With Enzalutamide and Abiraterone Resistance in Castration-Resistant Prostate Cancer. JCO Precis Oncol 2019;3.

40. Azad AA, Volik SV, Wyatt AW, et al. Androgen Receptor Gene Aberrations in Circulating Cell-Free DNA: Biomarkers of Therapeutic Resistance in CastrationResistant Prostate Cancer. Clin Cancer Res 2015;21:2315-24.

41. Annala M, Vandekerkhove G, Khalaf D, et al. Circulating Tumor DNA Genomics Correlate with Resistance to Abiraterone and Enzalutamide in Prostate Cancer. Cancer Discov 2018;8:444-57.

42. Wyatt AW, Azad AA, Volik SV, et al. Genomic Alterations in Cell-Free DNA and Enzalutamide Resistance in Castration-Resistant Prostate Cancer. JAMA Oncol 2016;2:1598-606.

43. Balbas MD, Evans MJ, Hosfield DJ, et al. Overcoming mutation-based resistance to antiandrogens with rational drug design. Elife 2013;2:e0499.

44. Joseph JD, Lu N, Qian J, et al. A clinically relevant androgen receptor mutation confers resistance to second-generation antiandrogens enzalutamide and ARN-509. Cancer Discov 2013;3:1020-9.

45. Moilanen AM, Riikonen R, Oksala R, et al. Discovery of ODM-201, a newgeneration androgen receptor inhibitor targeting resistance mechanisms to androgen signaling-directed prostate cancer therapies. Sci Rep 2015;5:12007.

46. Jernberg E, Bergh A, Wikstrom P. Clinical relevance of androgen receptor alterations in prostate cancer. Endocr Connect 2017;6:R146-R61.

47. Crona DJ, Whang YE. Androgen Receptor-Dependent and -Independent Mechanisms Involved in Prostate Cancer Therapy Resistance. Cancers (Basel) 2017;9. 48. Arora VK, Schenkein E, Murali R, et al. Glucocorticoid receptor confers resistance to antiandrogens by bypassing androgen receptor blockade. Cell 2013;155:1309-22.

49. Zhang T, Karsh LI, Nissenblatt MJ, Canfield SE. Androgen Receptor Splice Variant, AR-V7, as a Biomarker of Resistance to Androgen Axis-Targeted Therapies in Advanced Prostate Cancer. Clin Genitourin Cancer 2020;18:1-10.

50. Uo T, Plymate SR, Sprenger CC. The potential of AR-V7 as a therapeutic target. Expert Opin Ther Targets 2018;22:201-16.

51. Antonarakis ES, Lu C, Wang H, et al. AR-V7 and resistance to enzalutamide and abiraterone in prostate cancer. N Engl J Med 2014;371:1028-38.

52. Brown LC, Lu C, Antonarakis ES, Luo J, Armstrong AJ. Androgen receptor variant-driven prostate cancer II: advances in clinical investigation. Prostate Cancer Prostatic Dis 2020. 
53. Armstrong AJ, Halabi S, Luo J, et al. Prospective Multicenter Validation of Androgen Receptor Splice Variant 7 and Hormone Therapy Resistance in High-Risk Castration-Resistant Prostate Cancer: The PROPHECY Study. J Clin Oncol 2019;37:1120-9.

54. Abida W, Cyrta J, Heller G, et al. Genomic correlates of clinical outcome in advanced prostate cancer. Proc Natl Acad Sci U S A 2019;116:11428-36.

55. Ku SY, Rosario S, Wang Y, et al. Rb1 and Trp53 cooperate to suppress prostate cancer lineage plasticity, metastasis, and antiandrogen resistance. Science 2017;355:7883.

56. Mu P, Zhang Z, Benelli M, et al. SOX2 promotes lineage plasticity and antiandrogen resistance in TP53- and RB1-deficient prostate cancer. Science 2017;355:84-8.

57. Bishop JL, Thaper D, Vahid S, et al. The Master Neural Transcription Factor BRN2 Is an Androgen Receptor-Suppressed Driver of Neuroendocrine Differentiation in Prostate Cancer. Cancer Discov 2017;7:54-71.

58. Dardenne E, Beltran H, Benelli M, et al. N-Myc Induces an EZH2-Mediated Transcriptional Program Driving Neuroendocrine Prostate Cancer. Cancer Cell 2016;30:563-77.

59. Yin Y, Xu L, Chang Y, et al. N-Myc promotes therapeutic resistance development of neuroendocrine prostate cancer by differentially regulating miR421/ATM pathway. Mol Cancer 2019;18:11.

60. Beltran H, Prandi D, Mosquera JM, et al. Divergent clonal evolution of castration-resistant neuroendocrine prostate cancer. Nat Med 2016;22:298-305.

61. Aggarwal R, Huang J, Alumkal JJ, et al. Clinical and Genomic Characterization of Treatment-Emergent Small-Cell Neuroendocrine Prostate Cancer: A Multiinstitutional Prospective Study. J Clin Oncol 2018;36:2492-503.

62. Ku SY, Gleave ME, Beltran H. Towards precision oncology in advanced prostate cancer. Nat Rev Urol 2019;16:645-54.

63. Zhang Z, Zhou C, Li X, et al. Loss of CHD1 Promotes Heterogeneous

Mechanisms of Resistance to AR-Targeted Therapy via Chromatin Dysregulation.

Cancer Cell 2020;37:584-98 e11.

64. Clermont PL, Ci X, Pandha H, Wang Y, Crea F. Treatment-emergent neuroendocrine prostate cancer: molecularly driven clinical guidelines. Int J Endo Oncol 2019;6:IJE20.

65. Lang SH, Swift SL, White H, Misso K, Kleijnen J, Quek RGW. A systematic review of the prevalence of DNA damage response gene mutations in prostate cancer. Int J Oncol 2019;55:597-616.

66. Network NCC. NCCN Clinical Practice Guidelines in Oncology (NCCN Guidelines): Prostate Cancer. NCCN 2020; Version 2.2020.

67. Mateo J, Porta N, Bianchini D, et al. Olaparib in patients with metastatic castration-resistant prostate cancer with DNA repair gene aberrations (TOPARP-B): a multicentre, open-label, randomised, phase 2 trial. Lancet Oncol 2020;21:162-74. 68. Cheng HH, Pritchard CC, Boyd T, Nelson PS, Montgomery B. Biallelic Inactivation of BRCA2 in Platinum-sensitive Metastatic Castration-resistant Prostate Cancer. Eur Urol 2016;69:992-5. 
69. Zafeiriou Z, Bianchini D, Chandler R, et al. Genomic Analysis of Three Metastatic Prostate Cancer Patients with Exceptional Responses to Carboplatin Indicating Different Types of DNA Repair Deficiency. Eur Urol 2019;75:184-92.

70. Pomerantz MM, Spisak S, Jia L, et al. The association between germline BRCA2 variants and sensitivity to platinum-based chemotherapy among men with metastatic prostate cancer. Cancer 2017;123:3532-9.

71. Bilusic M, Madan RA, Gulley JL. Immunotherapy of Prostate Cancer: Facts and Hopes. Clin Cancer Res 2017;23:6764-70.

72. Abida W, Cheng ML, Armenia J, et al. Analysis of the Prevalence of Microsatellite Instability in Prostate Cancer and Response to Immune Checkpoint Blockade. JAMA Oncol 2019;5:471-8.

73. Nava Rodrigues D, Rescigno P, Liu D, et al. Immunogenomic analyses associate immunological alterations with mismatch repair defects in prostate cancer. J Clin Invest 2018;128:4441-53.

74. Marcus L, Lemery SJ, Keegan P, Pazdur R. FDA Approval Summary:

Pembrolizumab for the Treatment of Microsatellite Instability-High Solid Tumors. Clin Cancer Res 2019;25:3753-8.

75. FDA approves pembrolizumab for adults and children with TMB-H solid tumors. 2020. (Accessed July 1, 2020, at https://www.fda.gov/drugs/drug-approvalsand-databases/fda-approves-pembrolizumab-adults-and-children-tmb-h-solid-tumors.) 76. van Dessel LF, van Riet J, Smits M, et al. The genomic landscape of metastatic castration-resistant prostate cancers reveals multiple distinct genotypes with potential clinical impact. Nat Commun 2019;10:5251.

77. Schweizer MT, Ha G, Gulati R, et al. CDK12-Mutated Prostate Cancer: Clinical Outcomes With Standard Therapies and Immune Checkpoint Blockade. JCO Precis Oncol 2020;4:382-92.

78. Antonarakis ES, Velho PI, Fu W, et al. CDK12-Altered Prostate Cancer: Clinical Features and Therapeutic Outcomes to Standard Systemic Therapies, Poly (ADPRibose) Polymerase Inhibitors, and PD-1 Inhibitors. JCO Precis Oncol 2020;4:370-81. 79. Ferraldeschi R, Nava Rodrigues D, Riisnaes R, et al. PTEN protein loss and clinical outcome from castration-resistant prostate cancer treated with abiraterone acetate. Eur Urol 2015;67:795-802.

80. Adelaiye-Ogala R, Gryder BE, Nguyen YTM, et al. Targeting the PI3K/AKT pathway overcomes enzalutamide resistance by inhibiting induction of the glucocorticoid receptor. Mol Cancer Ther 2020.

81. Marques RB, Aghai A, de Ridder CMA, et al. High Efficacy of Combination Therapy Using PI3K/AKT Inhibitors with Androgen Deprivation in Prostate Cancer Preclinical Models. Eur Urol 2015;67:1177-85.

82. Carver BS, Chapinski C, Wongvipat J, et al. Reciprocal feedback regulation of $\mathrm{PI} 3 \mathrm{~K}$ and androgen receptor signaling in PTEN-deficient prostate cancer. Cancer Cell 2011;19:575-86.

83. Crumbaker M, Khoja L, Joshua AM. AR Signaling and the PI3K Pathway in Prostate Cancer. Cancers (Basel) 2017;9.

84. Gupta S, Li J, Kemeny G, et al. Whole Genomic Copy Number Alterations in Circulating Tumor Cells from Men with Abiraterone or Enzalutamide-Resistant Metastatic Castration-Resistant Prostate Cancer. Clin Cancer Res 2017;23:1346-57. 
85. Zhang Y, Xia M, Jin K, et al. Function of the c-Met receptor tyrosine kinase in carcinogenesis and associated therapeutic opportunities. Mol Cancer 2018;17:45.

86. Murillo-Garzon V, Kypta R. WNT signalling in prostate cancer. Nat Rev Urol 2017;14:683-96.

87. Isaacsson Velho $\mathrm{P}, \mathrm{Fu} \mathrm{W}$, Wang $\mathrm{H}$, et al. Wnt-pathway Activating Mutations Are Associated with Resistance to First-line Abiraterone and Enzalutamide in Castration-resistant Prostate Cancer. Eur Urol 2020;77:14-21.

88. Chen WS, Aggarwal R, Zhang L, et al. Genomic Drivers of Poor Prognosis and Enzalutamide Resistance in Metastatic Castration-resistant Prostate Cancer. Eur Urol 2019;76:562-71.

89. Semenza GL. Defining the role of hypoxia-inducible factor 1 in cancer biology and therapeutics. Oncogene 2010;29:625-34.

90. Semenza GL. Hypoxia-inducible factors in physiology and medicine. Cell 2012;148:399-408.

91. Schito L, Semenza GL. Hypoxia-Inducible Factors: Master Regulators of Cancer Progression. Trends Cancer 2016;2:758-70.

92. Stewart GD, Ross JA, McLaren DB, Parker CC, Habib FK, Riddick AC. The relevance of a hypoxic tumour microenvironment in prostate cancer. BJU Int 2010;105:8-13.

93. Bharti SK, Kakkad S, Danhier P, et al. Hypoxia Patterns in Primary and Metastatic Prostate Cancer Environments. Neoplasia 2019;21:239-46.

94. Mitani T, Harada N, Nakano Y, Inui H, Yamaji R. Coordinated action of hypoxia-inducible factor-1alpha and beta-catenin in androgen receptor signaling. J Biol Chem 2012;287:33594-606.

95. Mitani T, Yamaji R, Higashimura Y, Harada N, Nakano Y, Inui H. Hypoxia enhances transcriptional activity of androgen receptor through hypoxia-inducible factor1alpha in a low androgen environment. J Steroid Biochem Mol Biol 2011;123:58-64.

96. Fernandez EV, Reece KM, Ley AM, et al. Dual targeting of the androgen receptor and hypoxia-inducible factor 1alpha pathways synergistically inhibits castration-resistant prostate cancer cells. Mol Pharmacol 2015;87:1006-12.

97. Teo MY, Rathkopf DE, Kantoff P. Treatment of Advanced Prostate Cancer. Annu Rev Med 2019;70:479-99.

98. Scher HI, Morris MJ, Stadler WM, et al. Trial Design and Objectives for Castration-Resistant Prostate Cancer: Updated Recommendations From the Prostate Cancer Clinical Trials Working Group 3. J Clin Oncol 2016;34:1402-18.

99. Kapoor A, Wu C, Shayegan B, Rybak AP. Contemporary agents in the management of metastatic castration-resistant prostate cancer. Can Urol Assoc J 2016;10:E414-E23.

100. Caffo O, De Giorgi U, Fratino L, et al. Clinical Outcomes of Castration-resistant Prostate Cancer Treatments Administered as Third or Fourth Line Following Failure of Docetaxel and Other Second-line Treatment: Results of an Italian Multicentre Study. Eur Urol 2015;68:147-53.

101. de Wit R, de Bono J, Sternberg CN, et al. Cabazitaxel versus Abiraterone or Enzalutamide in Metastatic Prostate Cancer. N Engl J Med 2019;381:2506-18.

102. Khalaf DJ, Annala M, Taavitsainen S, et al. Optimal sequencing of enzalutamide and abiraterone acetate plus prednisone in metastatic castration-resistant prostate cancer: 
a multicentre, randomised, open-label, phase 2, crossover trial. Lancet Oncol 2019;20:1730-9.

103. Handy CE, Antonarakis ES. Sequencing Treatment for Castration-Resistant Prostate Cancer. Curr Treat Options Oncol 2016;17:64.

104. Morris M, Heller G, bryce AH, et al. Alliance A031201: A phase III trial of enzalutamide (ENZ) versus enzalutamide, abiraterone, and prednisone (ENZ/AAP) for metastatic castration resistant prostate cancer (mCRPC). J Clin Oncol 2019;37:5008.

105. Posadas EM, Chi KN, de Wit R, et al. Pharmacokinetics, Safety, and Antitumor Effect of Apalutamide with Abiraterone Acetate plus Prednisone in Metastatic Castration-Resistant Prostate Cancer: Phase 1b Study. Clin Cancer Res 2020. 106. Den RB, George D, Pieczonka C, McNamara M. Ra-223 Treatment for Bone Metastases in Castrate-Resistant Prostate Cancer: Practical Management Issues for Patient Selection. Am J Clin Oncol 2019;42:399-406.

107. Knechel MA, Schmidt KT, Figg WD. Combination treatment in metastatic castration-resistant prostate cancer: can we safely boost efficacy by adding radium-223? Cancer Biol Ther 2020;21:1-3.

108. Cursano MC, Iuliani M, Casadei C, et al. Combination radium-223 therapies in patients with bone metastases from castration-resistant prostate cancer: A review. Crit Rev Oncol Hematol 2020;146:102864.

109. Sweeney CJ, Gillessen S, Rathkopf D, et al. CT014 - IMbassador250: A phase III trial comparing atezolizumab with enzalutamide vs enzalutamide alone in patients with metastatic castration-resistant prostate cancer (mCRPC). AACR Annual Meeting; 2020; Virtual.

110. Mateo J, Carreira S, Sandhu S, et al. DNA-Repair Defects and Olaparib in Metastatic Prostate Cancer. N Engl J Med 2015;373:1697-708.

111. Abida W, Campbell D, Patnaik A, et al. PRELIMINARY RESULTS FROM THE TRITON2 STUDY OF RUCAPARIB IN PATIENTS (PTS) WITH DNA DAMAGE REPAIR (DDR)-DEFICIENT METASTATIC CASTRATIONRESISTANT PROSTATE CANCER (MCRPC): UPDATED ANALYSES. Ann Oncol 2019;30:v325-v55.

112. Lorente D, Olmos D, Mateo J, et al. Decline in Circulating Tumor Cell Count and Treatment Outcome in Advanced Prostate Cancer. Eur Urol 2016;70:985-92.

113. Scher HI, Graf RP, Schreiber NA, et al. Assessment of the Validity of NuclearLocalized Androgen Receptor Splice Variant 7 in Circulating Tumor Cells as a Predictive Biomarker for Castration-Resistant Prostate Cancer. JAMA Oncol 2018;4:1179-86.

114. Del Re M, Crucitta S, Sbrana A, et al. AR-V7 and AR-FL expression is associated with clinical outcome: a translational study in patients with castrate resistant prostate cancer. BJU Int 2019.

115. Antonarakis ES, Lu C, Luber B, et al. Androgen Receptor Splice Variant 7 and Efficacy of Taxane Chemotherapy in Patients With Metastatic Castration-Resistant Prostate Cancer. JAMA Oncol 2015;1:582-91.

116. Clark E, Morton M, Sharma S, et al. Prostate cancer androgen receptor splice variant 7 biomarker study - a multicentre randomised feasibility trial of biomarkerguided personalised treatment in patients with advanced prostate cancer (the VARIANT trial) study protocol. BMJ Open 2019;9:e034708. 
117. Gandhy SU, Karzai F, Marte J, et al. Evaluating biomarkers in metastatic castration-resistant prostate cancer patients treated with enzalutamide: PSA, circulating tumor cell counts, AR-V7 status and radiographic progression. J Clin Oncol 2020;38:abstr e17569.

118. West J, You L, Zhang J, et al. Towards Multidrug Adaptive Therapy. Cancer Res 2020;80:1578-89.

119. Zhang J, Cunningham JJ, Brown JS, Gatenby RA. Integrating evolutionary dynamics into treatment of metastatic castrate-resistant prostate cancer. Nat Commun 2017;8:1816.

120. West JB, Dinh MN, Brown JS, Zhang J, Anderson AR, Gatenby RA. Multidrug Cancer Therapy in Metastatic Castrate-Resistant Prostate Cancer: An Evolution-Based Strategy. Clin Cancer Res 2019;25:4413-21.

121. Schmidt KT, Chau CH, Price DK, Figg WD. Precision Oncology Medicine: The Clinical Relevance of Patient-Specific Biomarkers Used to Optimize Cancer Treatment. J Clin Pharmacol 2016;56:1484-99.

122. Bastos DA, Antonarakis ES. Galeterone for the treatment of advanced prostate cancer: the evidence to date. Drug Des Devel Ther 2016;10:2289-97.

123. Taplin ME, Antonarakis ES, Ferrante KJ, et al. Clinical factors associated with AR-V7 detection in ARMOR3-SV, a randomized trial of galeterone (Gal) vs enzalutamide (Enz) in men with AR-V7+ metastatic castration-resistant prostate cancer (mCRPC). J Clin Oncol 2017;35:5005-.

124. Le Moigne R, Zhou H, Obst JK, et al. Lessons learned from the metastatic castration-resistant prostate cancer phase I trial of EPI-506, a first-generation androgen receptor N-terminal domain inhibitor. J Clin Oncol 2019;37:257-.

125. Schweizer MT, Haugk K, McKiernan JS, et al. A phase I study of niclosamide in combination with enzalutamide in men with castration-resistant prostate cancer. PLoS One 2018;13:e0198389.

126. Madan RA, Schmidt KT, Karzai F, et al. Phase 2 Study of Seviteronel (INO-464) in Patients With Metastatic Castration-Resistant Prostate Cancer After Enzalutamide Treatment. Clin Genitourin Cancer 2020.

127. Yamamoto Y, Loriot Y, Beraldi E, et al. Generation 2.5 antisense oligonucleotides targeting the androgen receptor and its splice variants suppress enzalutamide-resistant prostate cancer cell growth. Clin Cancer Res 2015;21:1675-87. 128. Wang Y, Jiang X, Feng F, Liu W, Sun H. Degradation of proteins by PROTACs and other strategies. Acta Pharm Sin B 2020;10:207-38.

129. Rahbar K, Boegemann M, Yordanova A, et al. PSMA targeted radioligandtherapy in metastatic castration resistant prostate cancer after chemotherapy, abiraterone and/or enzalutamide. A retrospective analysis of overall survival. Eur J Nucl Med Mol Imaging 2018;45:12-9.

130. Teply BA, Wang H, Luber B, et al. Bipolar androgen therapy in men with metastatic castration-resistant prostate cancer after progression on enzalutamide: an open-label, phase 2, multicohort study. Lancet Oncol 2018;19:76-86.

131. Denmeade SR, Wang H, Cao H, et al. TRANSFORMER: Bipolar androgen therapy (BAT) versus enzalutamide (E) for castration-resistant metastatic prostate cancer (mCRPC). J Clin Oncol 2020;38:abstr. 5517. 
132. Beltran H, Oromendia C, Danila DC, et al. A Phase II Trial of the Aurora Kinase A Inhibitor Alisertib for Patients with Castration-resistant and Neuroendocrine Prostate Cancer: Efficacy and Biomarkers. Clin Cancer Res 2019;25:43-51.

133. Asangani IA, Wilder-Romans K, Dommeti VL, et al. BET Bromodomain Inhibitors Enhance Efficacy and Disrupt Resistance to AR Antagonists in the Treatment of Prostate Cancer. Mol Cancer Res 2016;14:324-31.

134. Welti J, Sharp A, Yuan W, et al. Targeting Bromodomain and Extra-Terminal (BET) Family Proteins in Castration-Resistant Prostate Cancer (CRPC). Clin Cancer Res 2018;24:3149-62.

135. Piha-Paul SA, Sachdev JC, Barve M, et al. First-in-Human Study of Mivebresib (ABBV-075), an Oral Pan-Inhibitor of Bromodomain and Extra Terminal Proteins, in Patients with Relapsed/Refractory Solid Tumors. Clin Cancer Res 2019;25:6309-19. 136. de Bono JS, De Giorgi U, Rodrigues DN, et al. Randomized Phase II Study Evaluating Akt Blockade with Ipatasertib, in Combination with Abiraterone, in Patients with Metastatic Prostate Cancer with and without PTEN Loss. Clin Cancer Res 2019;25:928-36.

137. Kolinsky MP, Rescigno P, Bianchini D, et al. A phase I dose-escalation study of enzalutamide in combination with the AKT inhibitor AZD5363 (capivasertib) in patients with metastatic castration-resistant prostate cancer. Ann Oncol 2020;31:619-25. 138. Graham L, Banda K, Torres A, et al. A phase II study of the dual mTOR inhibitor MLN0128 in patients with metastatic castration resistant prostate cancer. Invest New Drugs 2018;36:458-67.

139. Kelly WK, Halabi S, Carducci M, et al. Randomized, double-blind, placebocontrolled phase III trial comparing docetaxel and prednisone with or without bevacizumab in men with metastatic castration-resistant prostate cancer: CALGB 90401. J Clin Oncol 2012;30:1534-40.

140. Petrylak DP, Vogelzang NJ, Budnik N, et al. Docetaxel and prednisone with or without lenalidomide in chemotherapy-naive patients with metastatic castration-resistant prostate cancer (MAINSAIL): a randomised, double-blind, placebo-controlled phase 3 trial. Lancet Oncol 2015;16:417-25.

141. Ning YM, Gulley JL, Arlen PM, et al. Phase II trial of bevacizumab, thalidomide, docetaxel, and prednisone in patients with metastatic castration-resistant prostate cancer. J Clin Oncol 2010;28:2070-6.

142. Sonpavde GP, Pond GR, Fizazi K, et al. Cabozantinib for Progressive Metastatic Castration-resistant Prostate Cancer Following Docetaxel: Combined Analysis of Two Phase 3 Trials. Eur Urol Oncol 2018.

143. Agarwal N, Loriot Y, McGregor BA, et al. Cabozantinib in combination with atezolizumab in patients with metastatic castration-resistant prostate cancer: Results of cohort 6 of the COSMIC-021 study. J Clin Oncol 2020;38:abstr. 5564.

144. Schmidt KT, Peer CJ, Huitema ADR, et al. Measurement of NLG207 (formerly CRLX101) nanoparticle-bound and released camptothecin in human plasma. J Pharm Biomed Anal 2020;181:113073.

145. Karzai F, VanderWeele D, Madan RA, et al. Activity of durvalumab plus olaparib in metastatic castration-resistant prostate cancer in men with and without DNA damage repair mutations. J Immunother Cancer 2018;6:141. 


\section{TABLES}

Table 1: Key clinical trials leading to FDA-approved indications of SG-ARAs.

Table 2 - Frequency of select biomarkers associated with SG-ARA resistance.

Table 3 - Select phase III clinical trials optimizing current approved therapies for PCa with potential impact on SG-ARA acquired resistance.

Table 4 - Select clinical trials using investigational agents targeting pathways of SG-ARA acquired resistance.

Abbreviations: AAP, abiraterone acetate and prednisone; ADT, androgen deprivation therapy; APA, apalutamide; BID, twice daily; CI, confidence interval; DARO, darolutamide; DOCE, docetaxel; ENZA, enzalutamide; FG-ARA, first generation androgen receptor antagonist; HR, hazard ratio; HRR, homologous recombination repair; $\mathrm{mCSPC}$, metastatic castration-sensitive prostate cancer; $\mathrm{mCRPC}$, metastatic castration-resistant prostate cancer; MFS, metastasis-free survival; NR, not reported or evaluated; NSAA, non-steroidal anti-androgen; OS, overall survival; PFS, progressionfree survival; PSADT, prostate-specific antigen doubling time; QD, once daily; RA-223, radium-223; RAD, radiation therapy (doses unspecified in the tables); SBRT, stereotactic body radiation therapy; SG-ARA, second generation androgen receptor antagonist; TPP, time to PSA progression. Note: a "/" indicates an "or" clause in Tables 3 and 4. 
Table 1. Key clinical trials leading to FDA-approved indications of SG-ARAs.

\begin{tabular}{|c|c|c|c|c|c|c|}
\hline $\begin{array}{l}\text { Trial (NCT\#) } \\
\text { [additional } \\
\text { notes] }\end{array}$ & Comparison & Design & $\begin{array}{l}\text { rPFS (HR, } \\
{[95 \% \text { CI], }} \\
\text { p-value) }\end{array}$ & $\begin{array}{l}\text { TPP (HR, } \\
{[95 \% C I],} \\
\text { p-value) }\end{array}$ & $\begin{array}{l}\text { MFS (HR, } \\
{[95 \% C I],} \\
\text { p-value) }\end{array}$ & $\begin{array}{l}\text { OS (HR, } \\
{[95 \% C I],} \\
\text { p-value) }\end{array}$ \\
\hline \multicolumn{7}{|l|}{ mCRPC } \\
\hline $\begin{array}{l}\text { AFFIRM }^{9} \\
\text { (NCT00974311) } \\
\text { [after DOCE] }\end{array}$ & $\begin{array}{l}\text { ENZA } 160 \\
\text { mg QD } \\
(n=800) \text { vs. } \\
\text { Placebo } \\
(n=399)\end{array}$ & $\begin{array}{l}\text { Double-blind, } \\
\text { randomized, } \\
\text { placebo- } \\
\text { controlled }\end{array}$ & $\begin{array}{l}8.3 \text { vs. } 2.9 \\
\text { mos } \\
(0.40,[0.35- \\
0.47] \\
\text { p<0.001) }\end{array}$ & $\begin{array}{l}8.3 \text { vs. } 3.0 \\
\operatorname{mos}(0.25 \\
{[0.20-0.30]} \\
p<0.001)\end{array}$ & $\mathbf{N} / \mathbf{A}$ & $\begin{array}{l}18.4 \text { vs. } \\
13.6 \text { mos } \\
(0.63,[0.53- \\
0.75] \\
\text { p }<0.001)\end{array}$ \\
\hline $\begin{array}{l}\text { PREVAIL } \\
\text { (NC,21 } \\
\text { [before DOCE] }\end{array}$ & $\begin{array}{l}\text { ENZA 160 } \\
\text { mg QD } \\
(n=872) \text { vs. } \\
\text { Placebo } \\
(n=845) \\
\end{array}$ & $\begin{array}{l}\text { Double-blind, } \\
\text { randomized, } \\
\text { placebo- } \\
\text { controlled }\end{array}$ & $\begin{array}{l}20.0 \text { vs. } 5.4 \\
\operatorname{mos}(0.32 \\
{[0.28-0.36]} \\
p<0.0001)\end{array}$ & $\begin{array}{l}11.2 \text { vs. } 2.8 \\
\text { mos }(0.17, \\
{[0.15-0.20]} \\
p<0.001)\end{array}$ & $\mathbf{N} / \mathbf{A}$ & $\begin{array}{l}35.3 \text { vs. } \\
31.3 \text { mos } \\
(0.77,[0.67- \\
0.88], \\
p=0.0002)\end{array}$ \\
\hline \multicolumn{7}{|l|}{ mCSPC } \\
\hline $\begin{array}{l}\text { TITAN }^{17} \\
\text { (NCT02489318) } \\
\text { [Analysis at } 24 \\
\text { mos] }\end{array}$ & $\begin{array}{l}\text { APA } 240 \mathrm{mg} \\
\text { QD }(n=525) \\
\text { vs. Placebo } \\
(n=527)\end{array}$ & $\begin{array}{l}\text { Double-blind, } \\
\text { randomized, } \\
\text { placebo- } \\
\text { controlled }\end{array}$ & $\begin{array}{l}68.2 \% \text { vs. } \\
47.5 \% \\
(0.48,[0.39- \\
0.60] \\
p<0.001) \\
\end{array}$ & $\begin{array}{l}\text { NE vs. } 12.9 \\
\text { mos }(0.26, \\
{[0.21-0.31],} \\
\text { no p-value) }\end{array}$ & $\mathbf{N} / \mathbf{A}$ & $\begin{array}{l}82.4 \% \text { vs. } \\
73.5 \% \\
(0.67,[0.51- \\
0.89], \\
p=0.005)\end{array}$ \\
\hline $\begin{array}{l}\text { ARCHES }^{24} \\
\text { (NCT02677896) } \\
\text { [Analysis at } 24 \\
\text { mos] }\end{array}$ & $\begin{array}{l}\text { ENZA } 160 \\
\text { mg QD } \\
(n=574) \text { vs. } \\
\text { Placebo } \\
(n=576) \\
\end{array}$ & $\begin{array}{l}\text { Double-blind, } \\
\text { randomized, } \\
\text { placebo- } \\
\text { controlled }\end{array}$ & $\begin{array}{l}\text { NR vs. } 19.0 \\
\text { mos }(0.39 \\
{[0.30-0.50]} \\
p<0.001]\end{array}$ & $\begin{array}{l}\text { NR vs. NR } \\
(0.19, \\
{[0.13-0.26],} \\
p<0.001)\end{array}$ & $\mathbf{N} / \mathbf{A}$ & $\begin{array}{l}\text { Data } \\
\text { Maturing }\end{array}$ \\
\hline $\begin{array}{l}\text { ENZAMET }^{25} \\
\text { (NCT02446405) } \\
\text { [Analysis at } 36 \\
\text { mos] }\end{array}$ & $\begin{array}{l}\text { ENZA 160 } \\
\text { mg QD } \\
(n=563) \text { vs. } \\
\text { FG-ARA } \\
(n=562)\end{array}$ & $\begin{array}{l}\text { Open-label, } \\
\text { randomized, } \\
\text { FG-ARA- } \\
\text { controlled }\end{array}$ & $\begin{array}{l}68 \% \text { vs. } \\
41 \%(0.40, \\
{[0.33-0.49]} \\
p<0.0001)\end{array}$ & $\begin{array}{l}67 \% \text { vs. } \\
37 \%(0.39 \\
{[0.33-0.47]} \\
p<0.001)\end{array}$ & N/A & $\begin{array}{l}* 143 \text { vs. } \\
102 \text { deaths } \\
(0.67,[0.52- \\
0.86], \\
p=0.002)\end{array}$ \\
\hline \multicolumn{7}{|l|}{ nmCRPC } \\
\hline $\begin{array}{l}\text { SPARTAN }{ }^{17,32} \\
(\text { NCT01946204) }\end{array}$ & $\begin{array}{l}\text { APA } 240 \mathrm{mg} \\
\text { QD (n=806) } \\
\text { vs. Placebo } \\
(\mathrm{n}=401)\end{array}$ & $\begin{array}{l}\text { Double-blind, } \\
\text { randomized, } \\
\text { placebo- } \\
\text { controlled }\end{array}$ & $\begin{array}{l}40.5 \text { vs. } \\
14.7 \text { mos } \\
(0.29,[0.24- \\
0.36], \\
p<0.001)\end{array}$ & $\begin{array}{l}\text { NR vs. } 3.7 \\
\text { mos (0.06, } \\
\text { [0.05-0.08], } \\
\text { no p-value) }\end{array}$ & $\begin{array}{l}40.5 \text { vs. } \\
16.2 \text { mos } \\
(0.28,[0.23- \\
0.35], \\
p<0.0001)\end{array}$ & $\begin{array}{l}\text { NR vs. NR } \\
\text { mos (0.75, } \\
{[0.59-0.96]} \\
p=0.0197)\end{array}$ \\
\hline $\begin{array}{l}\text { ARAMIS } 16,33 \\
(\mathrm{NCT0200614)}\end{array}$ & $\begin{array}{l}\text { DARO } 600 \\
\text { mg BID } \\
(n=955) \mathrm{vs.} \\
\text { Placebo } \\
(n=554)\end{array}$ & $\begin{array}{l}\text { Double-blind, } \\
\text { randomized, } \\
\text { placebo- } \\
\text { controlled }\end{array}$ & $\begin{array}{l}36.8 \text { vs. } \\
14.8 \text { mos } \\
(0.38,[0.32- \\
0.45] \\
\text { p }<0.001)\end{array}$ & $\begin{array}{l}33.2 \text { vs. } 7.3 \\
\operatorname{mos}(0.13, \\
{[0.11-0.16]} \\
p<0.001)\end{array}$ & $\begin{array}{l}40.4 \text { vs. } \\
18.4 \text { mos } \\
(0.41,[0.34- \\
0.50], \\
\text { p }<0.001)\end{array}$ & $\begin{array}{l}\text { NR vs. NR } \\
\operatorname{mos}(0.69, \\
{[0.53-0.88]} \\
p=0.003)\end{array}$ \\
\hline $\begin{array}{l}\text { PROSPER }^{18,30} \\
(\text { NCT02003924) }\end{array}$ & $\begin{array}{l}\text { ENZA } 160 \\
\text { mg QD } \\
(n=933) \text { vs. } \\
\text { Placebo } \\
(n=468)\end{array}$ & $\begin{array}{l}\text { Double-blind, } \\
\text { randomized, } \\
\text { placebo- } \\
\text { controlled }\end{array}$ & $\begin{array}{l}\text { Not } \\
\text { reported }\end{array}$ & $\begin{array}{l}37.2 \text { vs. } 3.9 \\
\text { mos }(0.07 \\
{[0.05-0.08]} \\
p<0.001)\end{array}$ & $\begin{array}{l}36.6 \text { vs. } \\
14.7 \text { mos } \\
(0.29,[0.24- \\
0.35], \\
\text { p<0.001) }\end{array}$ & $\begin{array}{l}67.0 \text { vs. } \\
56.3 \text { mos } \\
(0.73,[0.61- \\
0.89], \\
\text { p=0.001) }\end{array}$ \\
\hline
\end{tabular}

Primary Endpoints are highlighted in Green. Data reported in percentages indicate the median was not reached at time of publication, thus it is reported as a percentage of patients with the indicated endpoint. *- Number of deaths per group at the time of analysis were reported 
Table 2. Frequency of select biomarkers associated with SG-ARA resistance.

\begin{tabular}{|c|c|c|c|c|}
\hline \multicolumn{2}{|c|}{ Genomic Alterations } & \multicolumn{2}{|c|}{ MSK-IMPACT } & SU2C $^{35}$ \\
\hline Pathway & Biomarker & \% in mCSPC & \% in mCRPC & \% in mCRPC \\
\hline AR Pathway & AR & $\mathbf{4 \%}$ & $\mathbf{5 2 \%}$ & $\mathbf{6 3 \%}$ \\
& FOXA1 & $\mathbf{1 0 \%}$ & $\mathbf{1 2 \%}$ & $\mathbf{1 2 \%}$ \\
& SPOP & $11 \%$ & $\mathbf{5 \%}$ & $\mathbf{8 \%}$ \\
& ETS fusions & - & - & $\mathbf{5 7 \%}$ \\
\hline Lineage Plasticity \& & TP53 & $\mathbf{1 8 \%}$ & $\mathbf{2 9} \%$ & $\mathbf{5 3 \%}$ \\
Neuroendocrine & RB1 & $\mathbf{7 \%}$ & $\mathbf{1 8 \%}$ & $\mathbf{9 \%}$ \\
Differentiation & CHD1 & - & - & $\mathbf{8 \%}$ \\
\hline DNA Damage Repair & BRCA2 & $\mathbf{7 \%}$ & $\mathbf{1 0 \%}$ & $\mathbf{1 4 \%}$ \\
& BRCA1 & $1 \%$ & $\mathbf{2 \%}$ & $\mathbf{1 \%}$ \\
& ATM & $\mathbf{2 \%}$ & $\mathbf{1 1 \%}$ & $\mathbf{7 \%}$ \\
& FANCA & $\mathbf{3 \%}$ & $\mathbf{7 \%}$ & - \\
& CDK12 & $\mathbf{6 \%}$ & $\mathbf{1 1 \%}$ & $\mathbf{5 \%}$ \\
& MSH2 & $\mathbf{2 \%}$ & $\mathbf{3 \%}$ & $\mathbf{2 \%}$ \\
& MLH1 & $\mathbf{1 \%}$ & $\mathbf{1 \%}$ & $\mathbf{1 \%}$ \\
\hline PI3K/AKT Pathway & PTEN & $\mathbf{1 8 \%}$ & $\mathbf{2 9} \%$ & $\mathbf{4 0 \%}$ \\
& PI3KCA & $\mathbf{4 \%}$ & $\mathbf{3 \%}$ & $\mathbf{5 \%}$ \\
& PI3KCB & - & - & $\mathbf{6 \%}$ \\
& PIK3R1 & $\mathbf{4 \%}$ & $\mathbf{5 \%}$ & $\mathbf{5 \%}$ \\
& AKT1 & $\mathbf{1 \%}$ & $\mathbf{1 \%}$ & $\mathbf{1 \%}$ \\
\hline APC & $\mathbf{1 4 \%}$ & $\mathbf{1 5 \%}$ & $\mathbf{9 \%}$ \\
Pathway & CTNN1 & $\mathbf{6 \%}$ & $\mathbf{3 \%}$ & $\mathbf{4 \%}$ \\
& RNF43 & $\mathbf{1 \%}$ & $\mathbf{3 \%}$ & $\mathbf{3 \%}$ \\
\hline
\end{tabular}

Percent frequency is based on analyses of the MSK-IMPACT and SU2C cohort analyses.

MSK-IMPACT included tumor specimens with matched normal blood from patients with mCSPC and treatment naïve $\mathrm{mCRPC}$

SU2C included tumor specimens with matched normal blood or buccal swabs from patients with mCRPC both prior to and after treatment with either AAP or ENZA 
Table 3. Select phase III clinical trials optimizing current approved therapies for PCa with potential impact on SG-ARA acquired resistance.

\begin{tabular}{|c|c|c|c|c|}
\hline $\begin{array}{l}\text { Study } \\
\text { Question }\end{array}$ & Trial Identifier & Treatment(s) & Population & Complete? \\
\hline \multirow[t]{3}{*}{$\begin{array}{l}\text { Treatment } \\
\text { Sequence }\end{array}$} & $\begin{array}{l}\text { CARD } \\
\text { NCT02485691 } \\
\end{array}$ & $\begin{array}{l}\text { Cabazitaxel vs. } \\
\text { AAP/ENZA }\end{array}$ & $\begin{array}{l}\text { mCRPC, post-DOCE + } \\
\text { AAP/ENZA }\end{array}$ & Yes \\
\hline & *NCT02125357 ${ }^{102}$ & $\begin{array}{l}A A P \rightarrow E N Z A \text { vs. } \\
\text { ENZA } \rightarrow \text { AAP }\end{array}$ & mCRPC, treatment naive & Yes \\
\hline & NCT04139772 & $\begin{array}{l}\text { DOCE vs. } \\
\text { AAP/ENZA }\end{array}$ & $\begin{array}{l}\text { Asymptomatic/oligometastatic } \\
\text { mCRPC, post-AAP/ENZA }\end{array}$ & No \\
\hline \multirow{8}{*}{$\begin{array}{l}\text { Earlier } \\
\text { Treatment } \\
\text { Indication }\end{array}$} & NCT03777982 & $\begin{array}{l}\text { ADT + AAP + APA vs. } \\
\text { ADT }\end{array}$ & $\begin{array}{l}\text { mCSPC with detectable PSA } \\
\text { after Radiation + minimal ADT }\end{array}$ & No \\
\hline & $\begin{array}{l}\text { DASL-HiCaP } \\
\text { NCT04136353 }\end{array}$ & $\begin{array}{l}\text { DARO + ADT + RAD } \\
\text { vs. } \\
\text { ADT + RAD } \\
\end{array}$ & High-risk localized PCa & No \\
\hline & NCT03488810 & $\begin{array}{l}\text { APA + ADT + RAD vs. } \\
\text { ADT + RAD }\end{array}$ & $\begin{array}{l}\text { Intermediate-limited high-risk } \\
\text { localized PCa }\end{array}$ & No \\
\hline & $\begin{array}{l}\text { ATLAS } \\
\text { NCT02531516 }\end{array}$ & $\begin{array}{l}\text { APA + ADT + RAD vs. } \\
\text { BICA + ADT + RAD }\end{array}$ & $\begin{array}{l}\text { High-risk localized or locally } \\
\text { advanced PCa }\end{array}$ & No \\
\hline & $\begin{array}{l}\text { ENZARAD } \\
\text { NCT02446444 }\end{array}$ & $\begin{array}{l}\text { ENZA + ADT + RAD vs. } \\
\text { NSAA + ADT + RAD }\end{array}$ & High-risk localized PCa & No \\
\hline & $\begin{array}{l}\text { PCS IX } \\
\text { NCT02685397 }\end{array}$ & $\begin{array}{l}\text { ENZA + ADT + SBRT } \\
\text { vs. ENZA + ADT } \\
\end{array}$ & mCRPC with oligometastases & No \\
\hline & NCT04134260 & $\begin{array}{l}\text { APA + AAP + ADT + } \\
\text { RAD vs. ADT + RAD } \\
\end{array}$ & Post-RP salvage therapy & No \\
\hline & *NCT03860987 & $\begin{array}{l}\text { ENZA + AAP + ADT + } \\
\text { DCFPyL Imaging } \rightarrow \text { RP }\end{array}$ & Intermediate to high-risk PCa & No \\
\hline \multirow[t]{7}{*}{$\begin{array}{l}\text { Combination } \\
\text { Therapy }\end{array}$} & $\begin{array}{l}\text { Alliance A0301201 } \\
\text { NCT01949337 }\end{array}$ & $\begin{array}{l}\text { ENZA + AAP vs. } \\
\text { ENZA (no difference) }\end{array}$ & mCRPC, treatment naïve & Yes \\
\hline & NCT02257736 & $\begin{array}{l}\text { APA + AAP vs. } \\
\text { ADT }\end{array}$ & mCRPC, chemotherapy naïve & No \\
\hline & $\begin{array}{l}\text { STAMPEDE Arm J } \\
\text { NCT00268476 }\end{array}$ & $\begin{array}{l}\text { ENZA + AAP + ADT vs. } \\
\text { ADT }\end{array}$ & mCSPC & No \\
\hline & NCT03009981 & $\begin{array}{l}\text { APA + AAP + ADT vs. } \\
\text { APA + ADT vs. ADT }\end{array}$ & nmCRPC, PSADT $\leq 9$ months & No \\
\hline & $\begin{array}{l}\text { ARASENS } \\
\text { NCT02799602 }\end{array}$ & $\begin{array}{l}\text { DARO + DOCE + ADT } \\
\text { vs. DOCE + ADT }\end{array}$ & mCSPC & No \\
\hline & $\begin{array}{l}\text { PEACE III } \\
\text { NCT02194842 } \\
\end{array}$ & $\begin{array}{l}\text { ENZA + RA-223 vs. } \\
\text { ENZA }\end{array}$ & mCRPC, treatment naïve & No \\
\hline & $\begin{array}{l}\text { ESCALATE } \\
\text { NCT04237584 } \\
\end{array}$ & $\begin{array}{l}\text { DARO/ENZA + RA-223 } \\
\text { vs. DARO/ENZA }\end{array}$ & mCRPC, treatment naïve & No \\
\hline \multirow[t]{6}{*}{$\begin{array}{l}\text { Precision } \\
\text { Medicine }\end{array}$} & $\begin{array}{l}\text { *TOPARP } \\
\text { NCT01682772 } \\
\end{array}$ & $\begin{array}{l}\text { Olaparib at } 400 \text { or } 300 \\
\text { mg BID }\end{array}$ & $\begin{array}{l}\text { HRR mutation enriched } \\
\text { mCRPC post-taxane }\end{array}$ & Yes \\
\hline & $\begin{array}{l}\text { PROfound } \\
\text { NCT02987543 }^{13}\end{array}$ & $\begin{array}{l}\text { Olaparib vs. } \\
\text { ENZA/AAP }\end{array}$ & $\begin{array}{l}\text { HRR mutation enriched } \\
\text { mCRPC post ENZA/AAP }\end{array}$ & Yes \\
\hline & $\begin{array}{l}\text { *TRITON2 } \\
\text { NCT02952534 }^{111}\end{array}$ & Rucaparib & $\begin{array}{l}\text { HRR mutation enriched } \\
\text { mCRPC post-DOCE }+ \\
\text { AAP/ENZA }\end{array}$ & Yes \\
\hline & $\begin{array}{l}\text { TRITON3 } \\
\text { NCT02975934 } \\
\end{array}$ & $\begin{array}{l}\text { Rucaparib vs. } \\
\text { AAP/ENZA/DOCE }\end{array}$ & $\begin{array}{l}\text { HRR mutation enriched } \\
\text { mCRPC post-AAP/ENZA }\end{array}$ & No \\
\hline & ISRCTN10246848 & $\begin{array}{l}\text { AR-V7 guided } \\
\text { AAP/ENZA or } \\
\text { DOCE/RA-223 vs. SOC }\end{array}$ & $\begin{array}{l}\text { mCRPC, post AAP/ENZA + } \\
\text { DOCE }\end{array}$ & No \\
\hline & $\begin{array}{l}\text { ProBio } \\
\text { NCT03903835 }\end{array}$ & $\begin{array}{l}\text { Biomarker guided - } \\
\text { AAP, ENZA, DOCE, } \\
\text { RA-223, Cabazitaxel or } \\
\text { Carboplatin }\end{array}$ & $\begin{array}{l}\text { mCRPC with }<3 \text { prior lines of } \\
\text { CRPC treatment }\end{array}$ & No \\
\hline \multirow[t]{2}{*}{$\begin{array}{l}\text { Adaptive } \\
\text { Therapy }\end{array}$} & *NCT02415621 ${ }^{119}$ & ^Adaptive AAP & $\begin{array}{l}\text { mCRPC, receiving first line } \\
\text { AAP }\end{array}$ & Yes \\
\hline & NCT03511196 & ^Adaptive AAP + ADT & mCSPC & No \\
\hline
\end{tabular}

*- not a phase III trial, all other trials are Phase III trials.

$\wedge$ - Treatment cessation and initiation determined by PSA trend, not radiographic progression

Italicized treatments are representative of the statistically superior treatments in completed trials 
Table 4. Select clinical trials using investigational agents targeting pathways of SG-ARA acquired resistance.

\begin{tabular}{|c|c|c|c|c|}
\hline $\begin{array}{l}\text { Drug Target or } \\
\text { Mechanism } \\
\end{array}$ & Trial Identifier & Agents & Population & Completed? \\
\hline \multicolumn{5}{|l|}{ AR Pathway } \\
\hline \multirow[t]{2}{*}{ CYP17-lyase } & $\begin{array}{l}\text { ARMOR3-SV } \\
\text { NCT02438007 }\end{array}$ & $\begin{array}{l}\text { Galeterone vs. } \\
\text { ENZA }\end{array}$ & AR-V7 positive mCRPC & Yes \\
\hline & NCT02130700 & Seviteronel (VT-464) & mCRPC, post-ENZA & Yes \\
\hline AR N-terminus & NCT02606123 ${ }^{124}$ & EPI-506 & $m C R P C \leq 2$ prior therapies & Yes \\
\hline AR-V7 degrader & NCT02532114 ${ }^{125}$ & Niclosamide + ENZA & mCRPC, post-AAP & Yes \\
\hline $\begin{array}{l}\text { AR antisense } \\
\text { oligonucleotide }\end{array}$ & NCT03300505 & $A R R x+$ ENZA & $\begin{array}{l}\text { mCRPC, no prior } \\
\text { chemo/ENZA for } \mathrm{mCRPC}\end{array}$ & No \\
\hline $\begin{array}{l}\text { AR degrader } \\
\text { (PROTAC) }\end{array}$ & NCT03888612 & $A R V-110$ & Progressive mCRPC & No \\
\hline PSMA & NCT03042468 & ${ }^{177} \mathrm{Lu}-P S M A-617$ & mCRPC, post-AAP/ENZA & No \\
\hline \multicolumn{5}{|c|}{ Alternative Hormonal Approaches } \\
\hline \multirow[t]{2}{*}{$\begin{array}{l}\text { Bipolar androgen } \\
\text { therapy (BAT) }\end{array}$} & $\begin{array}{l}\text { RESTORE } \\
\text { NCT02090114 } \\
\end{array}$ & $\begin{array}{l}\text { Cycling Testosterone with } \\
\text { AAP/ENZA }\end{array}$ & mCRPC, post-AAP/ENZA & No \\
\hline & $\begin{array}{l}\text { TRANSFORMER } \\
\text { NCT02286921 }^{131}\end{array}$ & $\begin{array}{l}\text { Testosterone vs. } \\
\text { ENZA }\end{array}$ & mCRPC, post-AAP & No \\
\hline GR & NCT03437941 & CORT125281 + ENZA & mCRPC, post-AAP/ENZA & No \\
\hline \multicolumn{5}{|c|}{ Lineage Plasticity and Neuroendocrine Differentiation } \\
\hline Aurora Kinase A & NCT01799278 ${ }^{132}$ & Alisertib & mCRPC, neuroendocrine & Yes \\
\hline \multirow[t]{2}{*}{ BET } & NCT02391480 ${ }^{135}$ & Mivebresib \pm venetoclax & Advanced solid tumors $^{\wedge}$ & Yes \\
\hline & NCT02711956 & ZEN003694 + ENZA & mCRPC, post-AAP/ENZA & No \\
\hline EZH2 & $\begin{array}{l}\text { ProSTAR } \\
\text { NCT03480646 } \\
\end{array}$ & CPI-1205 + AAP/ENZA & $\begin{array}{l}\text { mCRPC, post-AAP/SG- } \\
\text { ARA }\end{array}$ & No \\
\hline \multicolumn{5}{|l|}{ PI3K/AKT Pathway } \\
\hline \multirow[t]{2}{*}{ AKT } & NCT01485861 ${ }^{136}$ & $\begin{array}{l}\text { Ipatasertib + AAP vs. } \\
\text { AAP }\end{array}$ & mCRPC, post AAP/ENZA & No \\
\hline & NCT02525068 ${ }^{137}$ & $\begin{array}{l}\text { Capivasertib + ENZA vs. } \\
\text { ENZA }\end{array}$ & $\begin{array}{l}\text { mCRPC, post-AAP and } \\
\text { post-ENZA }\end{array}$ & No \\
\hline PI3K/mTOR & NCT02407054 & $\begin{array}{l}\text { LY3023414 + ENZA vs. } \\
\text { ENZA }\end{array}$ & mCRPC, post-AAP & Yes \\
\hline PI3K- $\beta$ & NCT02215096 & GSK2636771 + ENZA & $\begin{array}{l}\text { PTEN deficient mCRPC, } \\
\text { ENZA-naïve }\end{array}$ & Yes \\
\hline \multirow[t]{2}{*}{ mTOR } & NCT02091531 138 & MLN0128 & mCRPC & Yes \\
\hline & NCT02125084 & Everolimus + ENZA & mCRPC, SG-ARA naïve & No \\
\hline \multicolumn{5}{|c|}{ Angiogenesis, DNA Repair, and Immunotherapy } \\
\hline PD-L1 & $\begin{array}{l}\text { IMbassador250 } \\
\text { NCT03016312 }^{109} \\
\end{array}$ & $\begin{array}{l}\text { Atezolizumab + ENZA vs. } \\
\text { ENZA }\end{array}$ & mCRPC, post AAP & Yes \\
\hline \multirow[t]{2}{*}{ PD-1 } & $\begin{array}{l}\text { KEYNOTE-991 } \\
\text { NCT04191096 } \\
\end{array}$ & $\begin{array}{l}\text { Pembrolizumab + ENZA } \\
+ \text { ADT vs. ENZA + ADT } \\
\end{array}$ & mCSPC & No \\
\hline & $\begin{array}{l}\text { KEYNOTE-641 } \\
\text { NCT03834493 }\end{array}$ & $\begin{array}{l}\text { Pembrolizumab + ENZA } \\
\text { vs. } \\
\text { ENZA }\end{array}$ & $\begin{array}{l}\text { mCRPC, treatment naïve or } \\
\text { post AAP }\end{array}$ & No \\
\hline $\begin{array}{l}\text { MET/VEGFR2, } \\
\text { PD-L1 }\end{array}$ & NCT03170960 ${ }^{143}$ & $\begin{array}{l}\text { Cabozantinib }+ \\
\text { Atezolizumab } \\
\end{array}$ & mCRPC, post AAP/ENZA & No \\
\hline PD-L1, PARP & NCT02484404 $4^{145}$ & Durvalumab + Olaparib & mCRPC, post-AAP/ENZA & No \\
\hline PD-1, PARP & NCT03834519 & $\begin{array}{l}\text { Pembrolizumab }+ \\
\text { Olaparib vs. } A A P / E N Z A\end{array}$ & mCRPC, post-AAP/ENZA & No \\
\hline Top1, PARP & NCT02769962 & NLG207+ Olaparib & mCRPC, post-AAP/ENZA & No \\
\hline HIF-1 & NCT03531827 & NLG207 + ENZA & mCRPC, post-ENZA & No \\
\hline \multicolumn{5}{|c|}{ *Umbrella Trial Design } \\
\hline $\begin{array}{l}\text { AR, WEE1, cMET, } \\
\text { PLK4, AKT, } \\
\text { PD-L1/CTLA4 }\end{array}$ & NCT03385655 & $\begin{array}{l}\text { DARO, adavosertib, } \\
\text { savolitinib, CFI-400945, } \\
\text { ipatasertib, durvalumab }+ \\
\text { tremelimumab }\end{array}$ & $\begin{array}{l}\text { mCRPC, post- } \\
\text { AAP/ENZA/APA/DARO }\end{array}$ & No \\
\hline
\end{tabular}

^- A small cohort of patients with mCRPC were assessed in an expansion cohort

*- Umbrella Trials prospectively genotype patients then select the most optimal treatment based on biomarker signature. 


\section{Chapter 3}

\section{A Phase 2 Study of Seviteronel (INO-464) in Patients with Metastatic Castration- Resistant Prostate Cancer After Enzalutamide Treatment}

Ravi A. Madan ${ }^{1}$, Keith T. Schmidt ${ }^{2}$, Fatima Karzai ${ }^{1}$, Cody J. Peer ${ }^{2}$, Lisa M. Cordes ${ }^{1}$, Cindy H. Chau ${ }^{1}$, Seth M. Steinberg ${ }^{3}$, Helen Owens ${ }^{1}$, Joel Eisner ${ }^{4}$, William R. Moore ${ }^{4,5}$, William L. Dahut ${ }^{1}$, James L. Gulley ${ }^{1}$, William D. Figg ${ }^{1,2}$

${ }^{1}$ Genitourinary Malignancies Branch, Center for Cancer Research, National Cancer Institute, National Institutes of Health, Bethesda, MD

${ }^{2}$ Clinical Pharmacology Program, Office of the Clinical Director, Center for Cancer Research, National Cancer Institute, National Institutes of Health, Bethesda, MD

${ }^{3}$ Biostatistics and Data Management Section, Office of the Clinical Director, Center for Cancer Research, National Cancer Institute, National Institutes of Health, Bethesda, MD ${ }^{4}$ Innocrin Pharmaceuticals Inc., Durham, NC

${ }^{5}$ Vizuri Health Sciences, LLC., Fairfax, VA

Clin Genitourin Cancer. 2020 Aug;18(4):258-267 


\section{Abstract}

Background: Seviteronel was being developed by Innocrin Pharamceuticals as a selective cytochrome P450c17a (CYP17) 17,20-lyase (lyase) inhibitor and androgen receptor (AR) antagonist with activity against prostate cancer cells in vitro and in vivo. This open-label Phase II clinical study evaluated the tolerability and efficacy of seviteronel in patients with metastatic castration-resistant prostate cancer (mCRPC) previously treated with enzalutamide.

Experimental Design: Patients with mCRPC who previously progressed on enzalutamide were divided into two cohorts based on prior exposure to docetaxel.

Seviteronel was administered without routine oral steroids either twice daily with dose titration $(450 \mathrm{mg}$ ) or once daily without dose titration $(600 \mathrm{mg}$ or $750 \mathrm{mg})$. The primary objective was to determine the rate of significant PSA response (i.e. decline of $>50 \%$ ) following 12 weeks of seviteronel.

Results: Seventeen patients (median age: 71 [60 to 92]) were enrolled, with 8 patients having received prior docetaxel. Patients received a median of 2 cycles of treatment, with a majority of patients discontinuing treatment due to toxicity related to study drug. Most common adverse events included concentration impairment, fatigue, tremor and nausea. Despite changes in dosing, the study was closed prematurely due to magnitude of toxicity. One of seventeen patients $(6 \%)$ had a significant PSA decline.

Conclusions: Seviteronel was not generally well tolerated in patients with mCRPC who previously received enzalutamide and not associated with significant clinical reponses. Further investigation of single-agent seviteronel in this patient population is not 
warranted, however studies investigating seviteronel with low-dose dexamethasone are ongoing in patients with AR-postive tumors.

\section{MicroAbstract}

This open label Phase II clinical study evaluated the safety and efficacy of seviteronel (dosed once or twice daily without oral steroids) in patients with mCRPC previously treated with enzalutamide. The study was terminated early due to sub-optimal dosing strategies and significant CNS toxicity. Further evaluation of seviteronel is not warranted in this patient population on the basis of limited tolerability and insufficient clinical activity.

Keywords:

mCRPC, acquired resistance, CYP17 inhibitor, androgen receptor, enzalutamide 


\section{INTRODUCTION}

The recent introduction of highly potent and efficacious anti-hormonal therapies has improved the treatment landscape of patients with castration-resistant prostate cancer (CRPC). Since 2011, the FDA has approved three anti-hormonal agents for the treatment of CRPC: abiraterone acetate (AA), enzalutamide (ENZ), and apalutamide (APA) ${ }^{1-6} \mathrm{AA}$ is an irreversible and potent inhibitor of the 17,20 -lyase activity of cytochrome P450c17a (CYP17), blocking downstream production of androgens. However, AA also potently inhibits the 17-alpha-hydroxylase activity of CYP17, requiring the co-administration of oral steroids (e.g. prednisone) to reduce upstream steroid accumulation, cortisol suppression, and mineralocorticoid excess. ${ }^{7-9}$ AA has shown a significant overall survival (OS) advantage in patients with metastatic CRPC (mCRPC). ${ }^{1,3}$ ENZ and APA are both second generation androgen receptor (AR) antagonists that have also shown an OS improvement in patients with CRPC, the latter only in patients with non-metastatic CRPC (nmCRPC). ${ }^{2,4-6,10}$

Despite favorable responses in patients with castration resistance, disease progression is inevitable following treatment with current second-generation antihormonal agents, often a result of acquired resistance mediated via the AR pathway. ${ }^{11}$ Increased intratumoral androgen biosynthesis, AR overexpression, AR splice variation, and AR point mutations are implicated in treatment resistance to potent AR antagonists and CYP17 inhibitors. ${ }^{12-17}$ The T878A and L702H mutations in the AR have been associated with resistance to AA therapy, conferred via AR pathway activation by progesterone/pregnenolone ${ }^{9}$ and prednisone, ${ }^{18,19}$ respectively. Notably, the F877L mutation converts ENZ and APA from AR antagonists into AR agonists in vitro, with 
several cases documented clinically. ${ }^{20,21}$ Numerous clinical trials have been initiated to investigate newer antihormonal agents (e.g. orteronel, darolutamide, EPI-506) aimed to overcome AR pathway-mediated acquired resistance associated with the currently approved agents. $^{22}$

Seviteronel (INO-464) is an orally bioavailable, dual inhibitor of CYP17 lyase activity and the AR, with approximately 10-fold selectivity towards the CYP17 lyase over hydroxylase, ${ }^{23}$ and competitive inhibition of wild-type and mutated forms of the AR (e.g. T877A, F877L). ${ }^{24}$ The unique mechanism of action of seviteronel may offer a potential therapeutic option in the setting of prior AR-targeted treatment failure while sparing the use of concomitant steroids. Seviteronel was shown to be effective in several in vivo models using CRPC cell lines, including MR49F, MDA-PCA-133, and LNCaP (expressing the AR F877L, H874Y, T877A mutations respectively). ${ }^{24-26}$ Additionally, seviteronel was shown to be more potent than AA in established enzalutamide-resistant cell lines (e.g. C4-2, C4-2B, MR49C, MR49F). ${ }^{24,25}$

There is currently an unmet clinical need to improve treatments in the post-ENZ setting of mCRPC. Sequential use of AA following ENZ has shown minimal improvements in both progression-free survival (PFS) and OS due to AR-mediated cross-resistance, ${ }^{27}$ as evidenced by clinical biomarkers such as AR-V7 expression. ${ }^{11,13,14}$ The safety, tolerability, pharmacokinetics (PK), and, notably, preliminary clinical activity of seviteronel has been evaluated for both twice daily and once daily dosing regimens in patients with treatment-naïve and previously treated mCRPC (NCT02012920, NCT02361086). ${ }^{28,29}$ Patients with PSA declines were observed on both studies, but limited seviteronel tolerability associated with twice daily dosing (e.g. 
frequent dose reductions, treatment discontinuations) ultimately led to $600 \mathrm{mg}$ or 750 mg once daily dosing regimens. ${ }^{29}$ The current phase II study (NCT02130700) investigates the use of seviteronel in patients with progressive mCRPC who experienced disease progression following at least 3 months of ENZ monotherapy with and without prior exposure to cytotoxic chemotherapy.

\section{PATIENTS AND METHODS}

\section{Study Population}

Patients aged 18 years or older with progressive mCRPC previously treated with enzalutamide for greater than three 28-day cycles were eligible for this study.

Progression was defined as either a minimum of two rising PSA levels at least one week apart, appearance of one or more new lesions on bone scan, or new or growing lesions on CT scan. Patients were required to have an ECOG status of $\leq 1(\leq 2$ allowed for patients post-chemotherapy) with adequate organ and marrow function, castrate levels of testosterone ( $<50 \mathrm{ng} / \mathrm{dl}$, achieved via orchiectomy or continuous LHRH agonist/antagonist therapy), and have discontinued previous treatment at least 28 days prior to study entry.

Patients with an uncontrolled intercurrent illness, an HIV-positive diagnosis on combination antiretroviral therapy, active Hepatitis B or C infections, or a history of another invasive malignancy within the preceding 3 years were excluded from this study. No more than one prior course of cytotoxic chemotherapy was permitted, and only patients with prior cytotoxic chemotherapy may have received prior therapy with agents targeting CYP17 (e.g. abiraterone, galeterone, orteronel). Patients with adrenal 
insufficiency requiring daily hydrocortisone/prednisone or prior palliative radiation within 2 weeks of study entry were not eligible. Additionally, patients with known brain metastases or a history of seziures were excluded from this study.

\section{Study Design}

This was a Phase 2, open label study designed to explore the benefit of seviteronel in patients with $\mathrm{mCRPC}$ who have previously been treated with enzalutamide. The primary objective of this study was to determine the rate of significant PSA response as defined by a $\geq 50 \%$ decrease in baseline serum PSA after 12 weeks of seviteronel administration without routine oral steroids (per PCWG2 criteria). ${ }^{30}$ Patients were stratified into two cohorts: pre-docetaxel based chemotherapy or post-docetaxel based chemotherapy. In each cohort, a Simon optimal two-stage design was used, with alpha $=0.10$ and beta $=0.10$, to rule out a $5 \%$ response rate $\left(\mathrm{p}_{0}=0.05\right)$ in favor of a targeted $25 \%$ PSA response rate $\left(\mathrm{p}_{1}=0.25\right)$. The first stage of accrual would include 9 patients in each cohort (18 patients total, initially). A significant PSA response in 1 or more of 9 patients in a study arm would increase enrollment to a total of 24 patients in that arm, with 3 or more responses in 24 patients warranting further study. The secondary objective for this study was to determine the radiographic response and time to progression as per the modified RECIST 1.1 criteria. $^{31}$

\section{Treatment and Toxicity Evaluation}

Patients initially received seviteronel $150 \mathrm{mg}$ by mouth twice daily with titration in increments of $150 \mathrm{mg}$ every two weeks to a final dose of $450 \mathrm{mg}$ twice daily. After the results of a simultaneous trial became available, ${ }^{29}$ the protocol was amended to 
modify the dose and administration schedule of seviteronel to $750 \mathrm{mg}$ by mouth once daily in an effort to improve tolerability. Seven patients were treated with the original dosing regimen followed by six patients who received seviteronel $750 \mathrm{mg}$ by mouth once daily. Frequent dose reductions and treatment discontinuations led to an additional amendment, reducing the dose of seviteronel to $600 \mathrm{mg}$ by mouth once daily for the remaining 4 patients enrolled on study.

Adverse events were classified and graded according to the National Cancer Institute Common Toxicity Criteria for Adverse Events (CTCAE version 4.0). Treatment was held for a Grade 3 adverse event that was possibly, probably, or definitely related to seviteronel until resolution to Grade 1 or baseline. Dose reductions were allowed for low grade adverse events at the discretion of the investigator. Reescalation of the dose was not permitted and patients requiring more than 2 dose reductions permanently discontinued seviteronel. Seviteronel was permanently discontinued for a Grade 4 adverse event or a treatment delay of greater than 6 weeks.

\section{Pharmacokinetic Analysis}

To better understand the pharmacokinetices (PK) of seviteronel, a subset of men on this trial were provided with a single oral dose (600 or $750 \mathrm{mg}$ ) with food and had PK samples drawn to 48 hours after dose on the first day of cycles 1 and 2. For these patients, the day 2 dose was withheld, with daily dosing resuming on day 3 of cycles 1 and 2, and continued through each 28-day cycle. Blood for PK measurements was drawn into sodium heparin (green top; BD Biosciences, San Jose, CA) tubes, processed into plasma immediately, and stored frozen until bioanalytical analysis. Seviteronel plasma 
concentrations were measured using a validated liquid chromatography-tandem mass spectrometric assay with a lower limit of quantitation of $20 \mathrm{ng} / \mathrm{mL}$. PK parameters were calculated using noncompartmental methods by Phoenix WinNonlin 7.0 (Certara, Cary, $\mathrm{NC})$. Exposure-response analyses of ordered grades of AE were assessed by propotional odds models in R v3.5.

\section{Statistical Analyses}

The Kaplan-Meier method was used to evaluate time to progression and overall survival, separately by cohort as well as overall. Analyses of progression were done by evaluating time to PSA or radiographic progression (whichever came first); the analysis censored each patient's follow-up at their off-study date if they did not have either a PSA progression or radiographic progression noted on-study. The log-rank test was used to determine the statistical significance of the difference between pairs of Kaplan-Meier curves. The data cutoff for this analysis was February 8, 2019.

\section{RESULTS}

\section{Patient Characteristics}

A total of 17 patients with mCRPC were enrolled from April 2014 to August 2016. Baseline characteristics separated by cohort are presented in Table 1, with approximately half of the patients having previous exposure to docetaxel $(\mathrm{N}=9,53 \%)$. Most patients presented with high risk prostate cancer at time of initial diagnosis as demonstrated by Gleason Score (i.e. Gleason Score $\geq 8$; N=12, 71\%) and metastatic disease with bone involvement at baseline $(\mathrm{N}=16,94 \%)$. A majority of the patients had 
been treated previously with a first-generation androgen receptor antagonist (antiandrogens such as bicalutamide, flutamide, nilutamide; $\mathrm{N}=16,94 \%$ ) in addition to prior treatment with enzalutamide, as required by the study eligibility criteria. Thirteen patients $(76 \%)$ had been previously treated with immunotherapy, with 12 patients $(71 \%)$ receiving either investigational anti-cancer vaccines (e.g. PROSTVAC, PANVAC, METARP; $\mathrm{N}=8,47 \%)$ or FDA-approved sipuleucel-T ( $\mathrm{N}=4,24 \%)$. Additionally, 5 patients (29\%) had prior treatment with anti-angiogenic targeted agents, the most notable regimen comprising of bevacizumab, docetaxel and either thalidomide or lenalidomide $(\mathrm{N}=3,18 \%)$.

\section{Clinical Response}

Patients received a median of 2 cycles (range, 1 to 8 cycles), limiting clinical evaluation of PSA response and radiographic progression as per PCWG2, which recommends waiting at least 12 weeks before documenting response or progression. ${ }^{30}$ Of the 17 evaluable patients, 10 (59\%) patients discontinued therapy due to adverse events, 4 (24\%) patients due to disease progression, $2(12 \%)$ patients because of physician discretion, and $1(6 \%)$ patient due to intercurrent illness unrelated to study treatment. Of all patients, only one patient from the pre-chemotherapy cohort receiving $750 \mathrm{mg}$ once daily met the primary objective $(6 \%)$, with a maximal PSA decline of $88 \%$ achieved following 3 cycles of treatment and disease progression occurring after 6 months on-study. An additional patient in the pre-chemotherapy cohort, who received $750 \mathrm{mg}$ once daily with dose reduction to $450 \mathrm{mg}$ once daily during Cycle 1, exhibited a minimal decline in PSA (15\%) after 11 weeks of treatment before discontinuing due to 
toxicity. All remaining patients had rising PSA values on study, with 8 of those patients having documented PSA progression per PCWG2. Only four of 17 (24\%) patients underwent restaging for the indication of radiographic progression of disease, with only one patient having a secondary scan to confirm progression per PCWG2. ${ }^{30}$

Kaplan-Meier plots for time to PSA progression or radiographic progression and overall survival are provided in Figures 1 and 2, respectively. Median time to disease progression (mTDP), measured as either PSA progression or radiographic progression (whichever came first), was 3.5 months (95\% CI: 2.2 to 3.6 months). Patients without previous chemotherapy had a mTDP of 3.6 months (95\% CI: 3.2 to 4.6 months) and patients previously treated with docetaxel had a mTDP of 2.7 months (95\% CI: 1.6 to 3.5 months); though the log-rank assessment demonstrated a statistically significant difference in $\mathrm{mTDP}$ between cohorts $(\mathrm{p}=0.0096)$, this finding was not interpreted to be clinically meaningful. Median overall survival (mOS) for all patients was 13.4 months (95\% CI: 6.6 to 14.3 months). Patients with prior chemotherapy had a mOS of 13.0 months (95\% CI: 5.2 to 14.1 months) whereas patients with no prior chemotherapy had a mOS of 14.3 months (95\% CI: 5.6 to 27.6 months).

\section{Toxicity}

Grade 1, 2 and 3 adverse events that were probably, possibly or definitely related to study treatment reported in greater than $15 \%$ of the patients are listed in Table 2 . The most common adverse events included concentration impairment, fatigue, tremor, and nausea, each of which each occurred in $>50 \%$ of the patients. Reported Grade 3 adverse events at least possibly related to study treatment included concentration impairment, 
dizziness, nausea, hypotension, fall, and dehydration (not reported in Table 2, 2 patients [12\%] experienced Grade 3 dehydration). No Grade 4 adverse events related to the study treatment were reported. Only one patient experienced two Grade 4 adverse events on study, respiratory failure and pneumonitis, that were attributed unrelated and unlikely, respectively, due to likely association with existing comorbidities. Supplemental Table $\mathbf{1}$ describes the incidence of the most common adverse events by dosing group. Toxicities that occurred in $>50 \%$ of the all patients were mostly consistent across all dosing strategies with the exception of diarrhea, which was not reported in patients receiving $750 \mathrm{mg}$ once daily. Ultimately, nine patients had CNS toxicities (most commonly concentration impairment and fatigue) that contributed to their treatment discontinuation.

In addition to dose modifications, alternative toxicity management strategies were attempted in a small number of patients $(\mathrm{N}=5)$. The precise mechanism of the CNS toxicities associated with seviteronel is unclear but hormonal or steroidal alterations were proposed as potential factors. As a result, some patients received estrogen supplementation $(\mathrm{N}=3)$ or prednisone $(\mathrm{N}=2)$, either prophylactically or after symptom development. No symptomatic improvement was noted with either approach.

\section{Pharmacokinetics}

Of the 8 patients with PK samples obtained, 7 had a full PK time course in cycle 1, and 6 patients had full time courses in both cycles 1 and 2 for calculation of PK parameters. Seviteronel demonstrated an apparent monophasic elimination that began approximately 2 to 4 hours after administration of the dose. Patients who received 750 
mg demonstrated higher $\mathrm{C}_{\mathrm{MAX}}(P=.50)$ and $\mathrm{AUC}_{\mathrm{INF}}(P=.01)$ compared to those receiving $600 \mathrm{mg}$ on cycle 1 , day 1 , yet with comparable $\mathrm{T}_{\mathrm{MAX}}$, clearance, and half-life (Table 3). Figure 3 depicts mean plasma concentration-time curves for each cycle on each dose level, where steady-state (cycle 2, day 1) levels are higher than those at first dose as a result of extensive accumulation. Exposure-response analyses assessing the correlation of either $\mathrm{C}_{\mathrm{MAX}}$ or area under the plasma concentration versus time curve (AUC) to AE grade for concentration impairment, tremor, confusion, and nausea found no significant associations. This was most likely due to the narrow dose (i.e. exposure) range found in this small study.

\section{DISCUSSION/CONCLUSIONS}

Seviteronel, given both as twice-daily and once-daily regimens in the absence of oral steroid supplementation, was generally not well tolerated in this phase 2 study that assessed patients with mCRPC who previously progressed on enzalutamide. While the toxicity profile largely reflects what has been previously published in both patients with breast cancer ${ }^{32}$ and prostate cancer, ${ }^{29}$ the high prevalence of concentration impairment, per se, seen on this study has not been previously reported and was not expected based on preclinical toxicology data with this agent. It is worth noting, however, that the phase 1 study of seviteronel with once daily dosing was not devoid of CNS toxicity, with $71 \%$ of patients experiencing fatigue, $52 \%$ with dizziness and $33 \%$ with blurry vision, all of which could be different manifestations of CNS toxicity. ${ }^{29}$ In this study, fatigue was commonly associated with concentration impairment, and those adverse events could present with overlapping symptomatology. Concentration impairment 
occurred in 14 of 17 patients (82\%), with Grade 2 or higher occurring in 5 of those patients and it contributed to treatment discontinuation in nine patients. Additionally, concentration impairment frequently resulted in dose reductions or treatment cessation. Other toxicities occurring in greater than $50 \%$ of this patient population included fatigue, tremor, and nausea, for which fatigue was previously reported at a similar frequency. This study did not find a correlation between drug exposure and adverse event severity, however, these analyses were limited by a small sample size. Although these patients often were previously treated with immunotherapy and angiogenesis inhibitors on clinical trials, it is unlikely that those treatments contributed to the CNS toxicity seen.

The most common toxicities observed with seviteronel, which included those with apparent CNS origin, are also found in patients experiencing adrenal glucocorticoid insufficiency. ${ }^{33-35}$ This suggests that minor CYP17 hydroxylase inhibition may be present with seviteronel administration and to aid in ameliorating those toxicities, the co-administration of the glucocorticoid mimetic dexamethasone was investigated in other new and ongoing breast and prostate cancer studies. ${ }^{29,32}$ Though no Grade 4 adverse events attributable to seviteronel were reported on this study, nine Grade 3 adverse events did occur, including concentration impairment and dizziness. Limited tolerability greatly impacted the analysis of clinical endpoints, including radiographic response and time to disease progression. Only one of 17 patients on study $(6 \%)$ achieved the study's primary endpoint of a PSA decline greater than $50 \%$, although toxicity required multiple changes in dosing strategy, which limits the ability to evaluate the potential efficacy of seviteronel in this study. 
The PK analysis of seviteronel in the present study uncovered significant increases in AUC from first dose to steady-state. This phenomenon was observed with unpublished sponsor data when PK sampling stopped at $24 \mathrm{hr}$ at first dose and $8 \mathrm{hr}$ at steady-state, however this wasn't reported in either of the previously published studies that only reported first dose $\mathrm{PK} .^{29,32}$ The data collected on this trial was sampled out to $48 \mathrm{hr}$ in order to better estimate the elimination rate, which ultimately lead to a more accurate half-life estimate compared to previous analyses. ${ }^{29,32}$ With a mean first dose half-life of $13.9 \mathrm{hr}$ (range 7.9 - $23.5 \mathrm{hr}$ ), seviteronel was expected to accumulate $43 \%$ above first-dose with once daily dosing until it reached steady-state $(69.5 \mathrm{hr}$, or 2.9 days, to reach $97 \%$ steady-state). This half-life estimate was based on a $48-\mathrm{hr}$ sampling window and considered to be more accurate than prior PK studies that only sampled to $24 \mathrm{hr}$ on first dose or $8 \mathrm{hr}$ at steady-state $\left(\mathrm{T}_{1 / 2} \sim 6-9 \mathrm{hr}\right) .^{29,32}$ Steady-state dosenormalized $\mathrm{C}_{\mathrm{MAX}}$ was $20 \%$ higher than Cycle 1 (means: $6.79 \mathrm{ug} / \mathrm{L} / \mathrm{mg}$ vs $8.16 \mathrm{ug} / \mathrm{L} / \mathrm{mg}$; $\mathrm{p}=0.44$ ). Dose-normalized steady-state $\mathrm{AUC}_{\mathrm{TAU}}$ was on average $61 \%$ higher than first dose $\mathrm{AUC}_{\mathrm{INF}}(6$ of 6 patients with available data had increases; $56.7 \mathrm{hr} * \mathrm{ug} / \mathrm{L} / \mathrm{mg}$ vs 91.3 hr*ug/L/mg; $\mathrm{p}=0.001)$. This significant accumulation ( $61 \%$ by AUC) to steady-state supports the toxicity profile, especially with persistent CNS events that take several halflives to resolve.

Efforts to improve the seviteronel toxicity profile were ongoing through the duration of this study, as evidenced by two adjustments to the on-study dosing strategy. Drug accumulation was originally postulated as a contributing factor to increased toxicity, with higher trough concentrations mediating CNS related adverse events, prompting reduction in dosing frequency from twice daily to once daily. The separate 
phase I dose escalation study evaluating once daily dosing did not formally define a maximum tolerated dose (MTD), but suggested seviteronel could be given at $750 \mathrm{mg}$ or $600 \mathrm{mg}$ once daily. ${ }^{29}$ The adoption of once daily dosing of seviteronel $750 \mathrm{mg}$ on the present study showed a minimal improvement in tolerability. Further investigation with $600 \mathrm{mg}$ once daily provided a similar adverse event profile without a significant clinical response, suggesting that both doses for once daily administration were not truly viable, especially without oral steroid co-administration. It is worth noting that inter-individual variability in seviteronel disposition would not adequately explain these toxicities.

Clinical characteristics such as body weight and prandial status, the latter of which significantly impacts abiraterone bioavailability, ${ }^{36}$ do not require clinically meaningful seviteronel dose modifications. ${ }^{37}$ Like enzalutamide, seviteronel exhibits low interindividual variability in men with $\mathrm{mCRPC}$, with only body weight having a minimal impact on seviteronel clearance. ${ }^{37,38}$

Currently available clinical data in patients with mCRPC post-ENZ or post-AA may suggest a limited or absent therapeutic window for both twice daily and once daily dosing of single-agent seviteronel. Initial investigations of seviteronel $450 \mathrm{mg}$ given twice daily yielded several PSA declines of $50 \%$ or greater in 2 of 7 patients who had previously received $\mathrm{ENZ},{ }^{28}$ providing the initial rationale for the current study.

Cumulative assessment of once daily dosing regimens in 28 patients with mCRPC previously treated with AA or ENZ is associated with only two clinical responses (7\%), both at the $750 \mathrm{mg}$ dose: one patient previously treated with AA with a PSA decline of greater than $30 \%,{ }^{29}$ and the one clinical response reported in the current study. Total daily doses of $750 \mathrm{mg}$ or greater appear to be associated with PSA declines in patients 
with mCRPC previously treated with AA or ENZ, however, tolerability has greatly limited the duration of treatment and potentially clinical response. It is possible that a tolerable and efficacious dose of seviteronel is not achievable for a majority of patients with mCRPC following anti-hormonal therapy or chemotherapy, at least by utilizing currently established dosing strategies in the absence of oral steroid co-administration, which was thought to be an important attribute of seviteronel in its clinic development.

Another potential contributing factor to the limited clinical response is exposure to prior lines of treatment. In patients receiving sequential lines of standard of care treatments (i.e. docetaxel, AA, ENZ and cabazitaxel), reported PSA response rates associated with $2^{\text {nd }}, 3^{\text {rd }}$ and $4^{\text {th }}$ lines of treatment are $38 \%, 24 \%$, and $16 \%$ respectively. ${ }^{27}$ When specifically analyzing sequences of ENZ followed by AA, two studies reported PSA response rates of $3 \%$ and $8 \%,{ }^{39,40}$ which is similar to the currently evaluated treatment sequence of ENZ followed by seviteronel. PSA response rates in treatment naïve patients receiving seviteronel were $11 \%(\mathrm{~N}=26)$ and $33 \%(\mathrm{~N}=9)$ for twice daily dosing and once daily dosing respectively (daily cumulative doses of $600 \mathrm{mg}$ or greater). ${ }^{28,29}$ Importantly, an association with increased response rates in treatment naïve patients compared to pre-treated patients (33\% vs. $0 \%$ ) was shown in the Phase I study evaluating once daily dosing. ${ }^{29}$ The only significant PSA decline in the present study occurred in a patient not previously treated with chemotherapy. Available clinical data does not support the utility of sevitronel's unique mechanism of action in the post-ENZ setting. Though characterization of tumor alterations was not performed on this study, it is possible the role of AR point mutations with affinity to seviteronel was negligible, either due to a minimal role driving ENZ-resistant disease (e.g. T877A, H874Y) ${ }^{41}$ or 
limited prevelance based on previous clinical reports in patients with mCRPC post-ENZ (e.g. F877L). ${ }^{12,15,16,20}$ Moreover, seviteronel's proposed specificity for the CYP17 lyase activity, proposed to mitigate mineralocorticoid excess associated with AA, was overshadowed by intolerable CNS toxicity. The inability of seviteronel to produce robust clinical responses may be indicative of the drug's ineffectiveness to target acquired resistance in a generalized post-ENZ mCRPC patient population in addition to the drug's sub-optimal dose density resulting from limited tolerability.

This trial provides a cautionary tale as the field of prostate cancer looks to target an androgen receptor pathway that has mutated or is otherwise resistant to standard ARdirected therapies. In developing next-generation AR-targeted therapy, heretofore underappreciate neurocognitive toxicity may be a significant limitation despite promising preclinical rationale, as it was with seviteronel. Preclinical studies have previously demonstrated the capability of ENZ and APA to penetrate the blood-brain barrier. ${ }^{42}$ Furthermore, this is not the first agent targeting the AR which has suggested neurotoxicity. There have been several studies suggesting that ADT has been associated with some degree of cognitive decline. ${ }^{43}$ Enzalutamide has also been associated with severe fatigue in some patients and even seizures. ${ }^{44}$ Notably, an episode of seizure activity coupled with limited anti-tumor activity during Phase I evaluation terminated the development of the novel anti-androgen, BMS-641988. ${ }^{45}$ As further AR-targeting strategies are investigated, great care needs to be taken to monitor for off-target neurologic toxicity.

In conclusion, none of the dosing strategies implemented for seviteronel administration in the present study were well tolerated by patients with mCRPC 
previously treated with ENZ. The limited tolerability coupled with a clinically insignificant response rate does not support further development of seviteronel, especially without oral steroid co-administration, in patients with mCRPC.

\section{Clinical Practice Points}

- Improving the efficacy of available treatments for patients with mCRPC following either anti-hormonal therapy or chemotherapy is an important objective currently under clinical investigation.

- Seviteronel is an orally bioavailable, dual inhibitor of CYP17 lyase activity and the AR proposed to limit toxicity associated with mineralocorticoid excess and target AR pathway mediated resistance following treatment with ENZ or AA in patients with mCRPC.

- Seviteronel given via twice daily or once daily dosing in patients with mCRPC previously treated with ENZ and/or docetaxel was associated with dose limiting CNS toxicities and insignificant clinical response.

- This study highlights the importance of appropriate dose selection and welldesigned pharmacokinetic analyses, as AUC assessments demonstrated significant increases in drug exposure following one cycle of treatment compared to the first dose.

- Clinical experience with seviteronel highlights the potential for dose-limiting neurocognitive toxicity often associated with the development of newer ARtargeted therapies, especially in the setting of acquired resistance. 


\section{Acknowledgements}

We thank the nursing staff of National Cancer Institute and the fellows of the Genitourinary Malignancies Branch at National Cancer Institute for their care of our patients; Peraton for data management assistance. Most importantly, we appreciate the patients with cancer who enroll in investigational trials to advance the knowledge of this disease.

\section{Grant Support}

This work was supported by the Intramural Research Program of the Center for Cancer Research, National Cancer Institute, National Institutes of Health.

\section{Disclaimer}

The content of this publication does not necessarily reflect the views or policies of the Department of Health and Human Services, nor does mention of trade names, commercial products, or organization imply endorsement by the U.S. Government. 


\section{REFERENCES}

1. de Bono JS, Logothetis CJ, Molina A, et al: Abiraterone and increased survival in metastatic prostate cancer. N Engl J Med 364:1995-2005, 2011

2. Scher HI, Fizazi K, Saad F, et al: Increased survival with enzalutamide in prostate cancer after chemotherapy. N Engl J Med 367:1187-97, 2012

3. Ryan CJ, Smith MR, de Bono JS, et al: Abiraterone in metastatic prostate cancer without previous chemotherapy. N Engl J Med 368:138-48, 2013

4. Beer TM, Armstrong AJ, Rathkopf DE, et al: Enzalutamide in metastatic prostate cancer before chemotherapy. N Engl J Med 371:424-33, 2014

5. Smith MR, Saad F, Chowdhury S, et al: Apalutamide Treatment and Metastasisfree Survival in Prostate Cancer. N Engl J Med 378:1408-1418, 2018

6. Rathkopf DE, Scher HI: Apalutamide for the treatment of prostate cancer. Expert Rev Anticancer Ther 18:823-836, 2018

7. Attard G, Reid AH, A'Hern R, et al: Selective inhibition of CYP17 with abiraterone acetate is highly active in the treatment of castration-resistant prostate cancer. J Clin Oncol 27:3742-8, 2009

8. Attard G, Reid AH, Yap TA, et al: Phase I clinical trial of a selective inhibitor of CYP17, abiraterone acetate, confirms that castration-resistant prostate cancer commonly remains hormone driven. J Clin Oncol 26:4563-71, 2008

9. Attard G, Reid AH, Auchus RJ, et al: Clinical and biochemical consequences of CYP17A1 inhibition with abiraterone given with and without exogenous glucocorticoids in castrate men with advanced prostate cancer. J Clin Endocrinol Metab 97:507-16, 2012

10. Hussain M, Fizazi K, Saad F, et al: Enzalutamide in Men with Nonmetastatic, Castration-Resistant Prostate Cancer. N Engl J Med 378:2465-2474, 2018

11. Robinson D, Van Allen EM, Wu YM, et al: Integrative clinical genomics of advanced prostate cancer. Cell 161:1215-1228, 2015

12. Annala M, Vandekerkhove G, Khalaf D, et al: Circulating Tumor DNA Genomics Correlate with Resistance to Abiraterone and Enzalutamide in Prostate Cancer. Cancer Discov 8:444-457, 2018

13. Antonarakis ES, $\mathrm{Lu} \mathrm{C}$, Wang $\mathrm{H}$, et al: AR-V7 and resistance to enzalutamide and abiraterone in prostate cancer. N Engl J Med 371:1028-38, 2014

14. Antonarakis ES, Lu C, Luber B, et al: Clinical Significance of Androgen Receptor Splice Variant-7 mRNA Detection in Circulating Tumor Cells of Men With Metastatic Castration-Resistant Prostate Cancer Treated With First- and Second-Line Abiraterone and Enzalutamide. J Clin Oncol 35:2149-2156, 2017

15. Azad AA, Volik SV, Wyatt AW, et al: Androgen Receptor Gene Aberrations in Circulating Cell-Free DNA: Biomarkers of Therapeutic Resistance in CastrationResistant Prostate Cancer. Clin Cancer Res 21:2315-24, 2015

16. Wyatt AW, Azad AA, Volik SV, et al: Genomic Alterations in Cell-Free DNA and Enzalutamide Resistance in Castration-Resistant Prostate Cancer. JAMA Oncol 2:1598-1606, 2016

17. Liu C, Lou W, Zhu Y, et al: Intracrine Androgens and AKR1C3 Activation Confer Resistance to Enzalutamide in Prostate Cancer. Cancer Res 75:1413-22, 2015 
18. Cai C, Balk SP: Intratumoral androgen biosynthesis in prostate cancer pathogenesis and response to therapy. Endocr Relat Cancer 18:R175-82, 2011

19. Boudadi K, Antonarakis ES: Resistance to Novel Antiandrogen Therapies in Metastatic Castration-Resistant Prostate Cancer. Clin Med Insights Oncol 10:1-9, 2016 20. Joseph JD, Lu N, Qian J, et al: A clinically relevant androgen receptor mutation confers resistance to second-generation antiandrogens enzalutamide and ARN-509. Cancer Discov 3:1020-9, 2013

21. Balbas MD, Evans MJ, Hosfield DJ, et al: Overcoming mutation-based resistance to antiandrogens with rational drug design. Elife 2:e00499, 2013

22. Yoo S, Choi SY, You D, et al: New drugs in prostate cancer. Prostate Int 4:3742, 2016

23. Rafferty SW, Eisner JR, Moore WR, et al: Highly-selective 4-(1,2,3-triazole)based P450c17a 17,20-lyase inhibitors. Bioorg Med Chem Lett 24:2444-7, 2014 24. Norris JD, Ellison SJ, Baker JG, et al: Androgen receptor antagonism drives cytochrome P450 17A1 inhibitor efficacy in prostate cancer. J Clin Invest 127:23262338, 2017

25. Toren PJ, Kim S, Pham S, et al: Anticancer activity of a novel selective CYP17A1 inhibitor in preclinical models of castrate-resistant prostate cancer. Mol Cancer Ther 14:59-69, 2015

26. Maity SN, Titus MA, Gyftaki R, et al: Targeting of CYP17A1 Lyase by VT-464 Inhibits Adrenal and Intratumoral Androgen Biosynthesis and Tumor Growth of Castration Resistant Prostate Cancer. Sci Rep 6:35354, 2016

27. Caffo O, De Giorgi U, Fratino L, et al: Clinical Outcomes of Castration-resistant Prostate Cancer Treatments Administered as Third or Fourth Line Following Failure of Docetaxel and Other Second-line Treatment: Results of an Italian Multicentre Study. Eur Urol 68:147-53, 2015

28. de Bono JS, Pezaro CJ, Gillessen S, et al: The oral CYP17-Lyase (L) inhibitor VT-464 in patients with CRPC. J Clin Oncol 33(suppl 7):abstr 187, 2015

29. Gupta S, Nordquist LT, Fleming MT, et al: Phase 1 Study of Seviteronel, a Selective CYP17 Lyase and Androgen Receptor Inhibitor, in Men with CastrationResistant Prostate Cancer. Clin Cancer Res, 2018

30. Scher HI, Halabi S, Tannock I, et al: Design and end points of clinical trials for patients with progressive prostate cancer and castrate levels of testosterone: recommendations of the Prostate Cancer Clinical Trials Working Group. J Clin Oncol 26:1148-59, 2008

31. Schwartz LH, Litiere S, de Vries E, et al: RECIST 1.1-Update and clarification: From the RECIST committee. Eur J Cancer 62:132-7, 2016

32. Bardia A, Gucalp A, DaCosta N, et al: Phase 1 study of seviteronel, a selective CYP17 lyase and androgen receptor inhibitor, in women with estrogen receptor-positive or triple-negative breast cancer. Breast Cancer Res Treat, 2018

33. Charmandari E, Nicolaides NC, Chrousos GP: Adrenal insufficiency. Lancet 383:2152-67, 2014

34. Michels A, Michels N: Addison disease: early detection and treatment principles. Am Fam Physician 89:563-8, 2014 
35. DL L, AS F, DL K, et al: Harrison's Principles of Internal Medicine, 18th Ed. New York, McGraw-Hill, 2012

36. Szmulewitz RZ, Peer CJ, Ibraheem A, et al: Prospective International Randomized Phase II Study of Low-Dose Abiraterone With Food Versus Standard Dose Abiraterone In Castration-Resistant Prostate Cancer. J Clin Oncol 36:1389-1395, 2018 37. Peer CJ, Schmidt KT, Kindrick JD, et al: A population pharmacokinetic analysis of the oral CYP17 lyase and androgen receptor inhibitor seviteronel in patients with advanced/metastatic castration-resistant prostate cancer or breast cancer. Cancer Chemother Pharmacol 84:759-770, 2019

38. Gibbons JA, Ouatas T, Krauwinkel W, et al: Clinical Pharmacokinetic Studies of Enzalutamide. Clin Pharmacokinet 54:1043-55, 2015

39. Noonan KL, North S, Bitting RL, et al: Clinical activity of abiraterone acetate in patients with metastatic castration-resistant prostate cancer progressing after enzalutamide. Ann Oncol 24:1802-7, 2013

40. Loriot Y, Bianchini D, Ileana E, et al: Antitumour activity of abiraterone acetate against metastatic castration-resistant prostate cancer progressing after docetaxel and enzalutamide (MDV3100). Ann Oncol 24:1807-12, 2013

41. McCrea E, Sissung TM, Price DK, et al: Androgen receptor variation affects prostate cancer progression and drug resistance. Pharmacol Res 114:152-162, 2016 42. Moilanen AM, Riikonen R, Oksala R, et al: Discovery of ODM-201, a newgeneration androgen receptor inhibitor targeting resistance mechanisms to androgen signaling-directed prostate cancer therapies. Sci Rep 5:12007, 2015

43. Nelson CJ, Lee JS, Gamboa MC, et al: Cognitive effects of hormone therapy in men with prostate cancer: a review. Cancer 113:1097-106, 2008

44. Beer TM, Armstrong AJ, Rathkopf D, et al: Enzalutamide in Men with Chemotherapy-naive Metastatic Castration-resistant Prostate Cancer: Extended Analysis of the Phase 3 PREVAIL Study. Eur Urol 71:151-154, 2017

45. Rathkopf D, Liu G, Carducci MA, et al: Phase I dose-escalation study of the novel antiandrogen BMS-641988 in patients with castration-resistant prostate cancer. Clin Cancer Res 17:880-7, 2011 


\section{FIGURE LEGENDS}

Figure 1. Time to PSA or Radiographic Progression. Kaplan-Meier plots of time to PSA or radiographic progression of the total population (A) and separated by patients with or without prior docetaxel therapy (B).

Figure 2. Median Overall Survival. Kaplan-Meier plots of overall survival of the total population (A) and separated by patients with or without prior docetaxel therapy (B). Median potential follow-up was 37.4 months.

Figure 3. Mean Seviteronel Plasma Concentration vs Time Curves by Dose and Cycle. 
Figure 1A

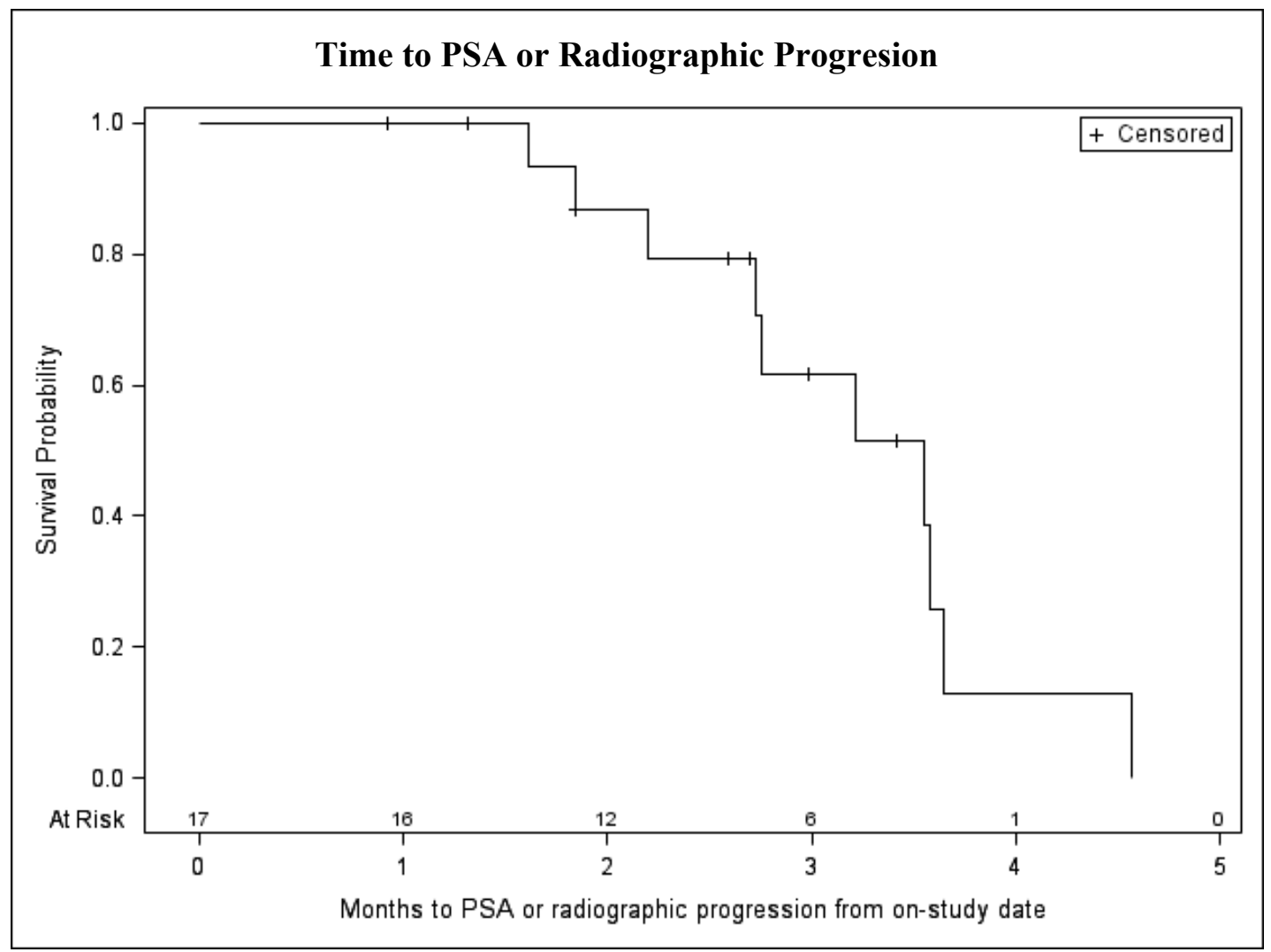

All patients

Median time to PSA or radiographic progression: 3.5 months ( $95 \% \mathrm{Cl}: 2.2-3.6$ months) 
Figure 1B

Time to PSA or Radiographic Progresion Based on Previous Chemotherapy

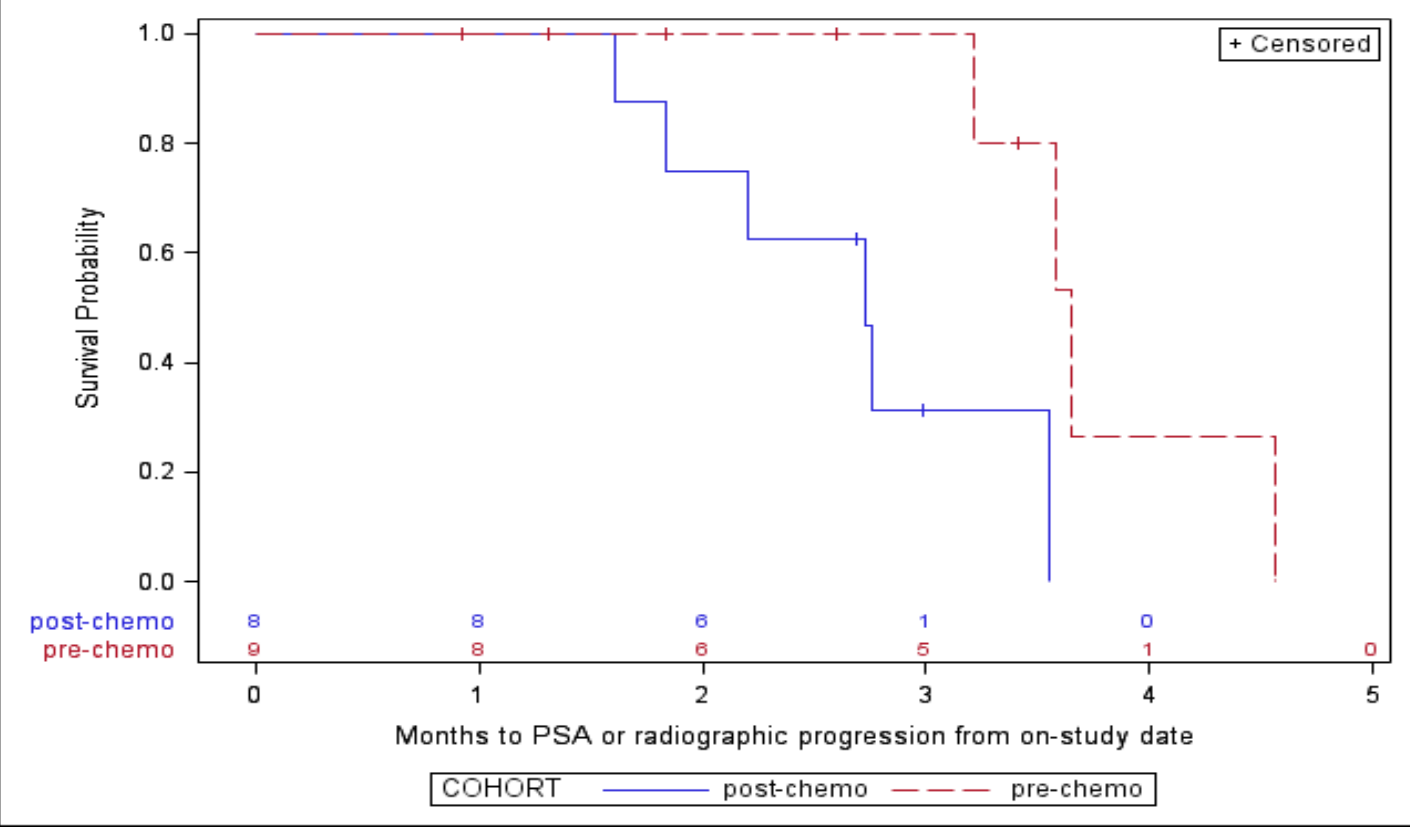

Median time to PSA or radiographic progression:

Post-chemo: 2.7 months (1.6 - 3.5 months)

Pre-chemo: 3.6 months (3.2-4.6 months)

Two-tailed log-rank $p=0.0096$ 
Figure 2A

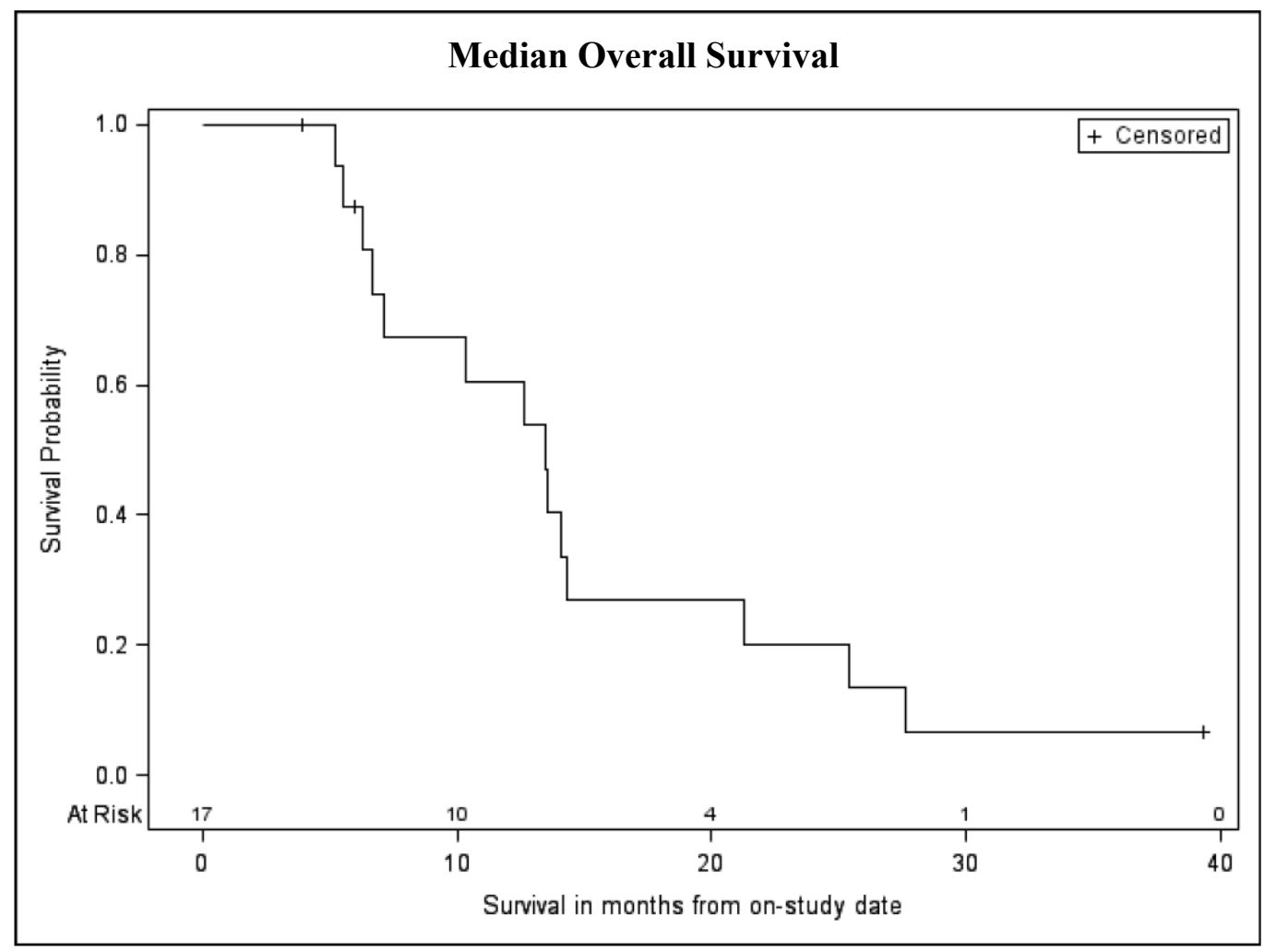

All patients:

Median OS: 13.4 months (95\% Cl: $6.6-14.3$ months) 
Figure 2B

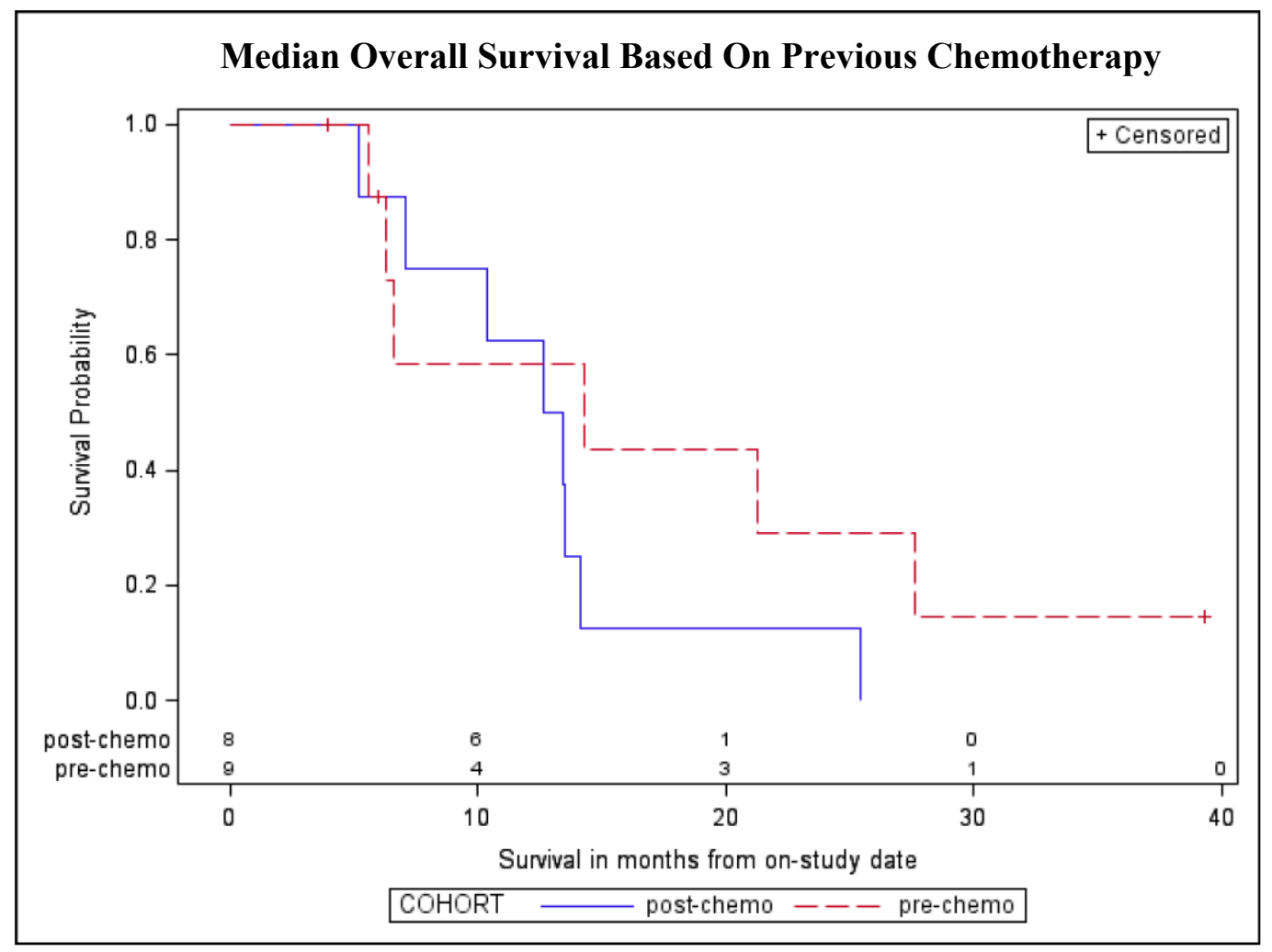

Median OS:

Post-chemo: 13.0 months ( $95 \% \mathrm{Cl}: 5.2-14.1$ months)

Pre-chemo: 14.3 months (95\% Cl: $5.6-27.6$ months)

Two-tailed log-rank $p=0.23$ 
Figure 3

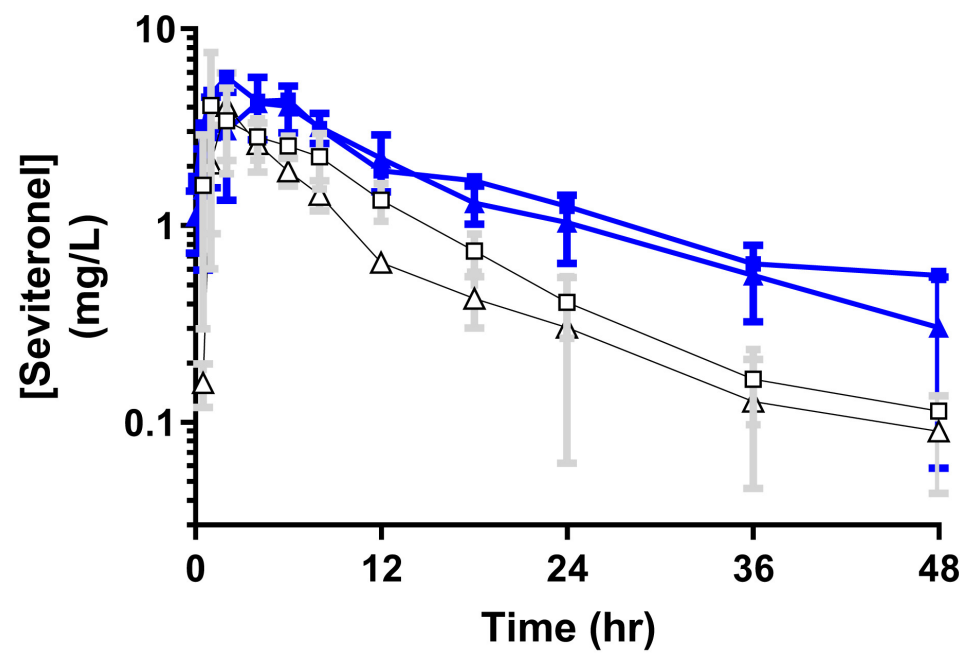

$\rightarrow-750 \mathrm{mg} \mathrm{C1D1}$

$\rightarrow 750 \mathrm{mg}$ C2D1

$\triangle 600$ mg C1D1

$\leftarrow \quad 600 \mathrm{mg} \mathrm{C} 2 \mathrm{D} 1$

Time (hr) 
Table 1. Patient Demographics and Baseline Characteristics

\begin{tabular}{|c|c|c|c|}
\hline & Pre-docetaxel & Post-docetaxel & Total \\
\hline & $\mathbf{N}=\mathbf{9}$ & $\mathbf{N}=\mathbf{8}$ & $\mathbf{N}=\mathbf{1 7}$ \\
\hline Age (years) $)^{\#}$ & $71(60,85)$ & $72(65,92)$ & $71(60,92)$ \\
\hline \multicolumn{4}{|l|}{ Race } \\
\hline White & $7(78)$ & $8(100)$ & $15(88)$ \\
\hline Other & $2(22)$ & $\mathbf{0}(\mathbf{0})$ & $2(12)$ \\
\hline Weight $(\mathbf{k g})^{\#}$ & $\begin{array}{c}96.5 \\
(76.6,129.4)\end{array}$ & $\begin{array}{c}89.1 \\
(74.3,112.7)\end{array}$ & $\begin{array}{c}93.0 \\
(74.3,129.4)\end{array}$ \\
\hline \multicolumn{4}{|l|}{ ECOG Status } \\
\hline 0 & $2(22)$ & $\mathbf{0}(\mathbf{0})$ & $2(12)$ \\
\hline 1 & $7(78)$ & $7(87.5)$ & $14(82)$ \\
\hline 2 & $\mathbf{0}(\mathbf{0})$ & $1(12.5)$ & $1(6)$ \\
\hline Baseline PSA & $\begin{array}{c}36.13 \\
(6.92,69.23)\end{array}$ & $\begin{array}{c}75.97 \\
(14.69,190)\end{array}$ & $\begin{array}{c}54.88 \\
(6.92,190)\end{array}$ \\
\hline \multicolumn{4}{|l|}{ Site of Metastasis } \\
\hline Bone & $3(33)$ & $5(62.5)$ & $8(48)$ \\
\hline Lymph Node (LN) & $1(11)$ & 0 (0) & $1(6)$ \\
\hline Bone and $L N$ & $4(45)$ & $\mathbf{0}(\mathbf{0})$ & $4(24)$ \\
\hline Bone and Visceral & $1(11)$ & $3(37.5)$ & $4(24)$ \\
\hline \multicolumn{4}{|l|}{ Gleason Score $^{\wedge}$} \\
\hline 6 & $1(11)$ & $1(12.5)$ & $2(12)$ \\
\hline 7 & $1(11)$ & $2(25)$ & $3(17)$ \\
\hline $8-10$ & $7(78)$ & $5(62.5)$ & $12(71)$ \\
\hline \multicolumn{4}{|l|}{ Prior Treatments" } \\
\hline Bicalutamide & $7(78)$ & 7 (87.5) & $14(82)$ \\
\hline Nilutamide & $2(22)$ & $3(37.5)$ & $5(29)$ \\
\hline Flutamide & $3(33)$ & $4(50)$ & $7(41)$ \\
\hline Ketoconazole & $2(22)$ & $2(25)$ & $4(24)$ \\
\hline Immunotherapy $^{+}$ & $7(77)$ & $6(75)$ & $13(76)$ \\
\hline $\begin{array}{l}\text { Anti-angiogenic } \\
\text { Therapy }^{\&}\end{array}$ & $0(0)$ & $5(62.5)$ & $5(29)$ \\
\hline \multicolumn{4}{|c|}{$\begin{array}{l}\text { All values are reported as total (percent) per column with unless otherwise noted. } \\
\text { \#- Reported as an average value and range. } \\
\text {-- Gleason Score that was reported at the time of diagnosis } \\
\text { - - Prior treatments other than enzalutamide or docetaxel } \\
\text { +- Treatments include: Anti-PD-1/PD-L1 antibodies, sipuleucel-T, PANVAC, PROSTVAC, and } \\
\text { TARP } \\
\&_{-} \text {-Treatment regimens containing the following agents: TRC-105, AMG386, thalidomide, } \\
\text { lenalidomide, bevacizumab. }\end{array}$} \\
\hline
\end{tabular}


Table 2. Adverse Events with an Attribution of at Least Possible Occurring in Greater than $15 \%$ of Patients Who Received Study Treatment $(\mathrm{N}=17)$ Based on National Cancer Institute Common Terminology Criteria for Adverse Events (Version 4.0)

\begin{tabular}{|c|c|c|c|c|}
\cline { 2 - 5 } \multicolumn{1}{c|}{} & \multicolumn{4}{c|}{ No. Patients (\%) } \\
\hline Adverse Event & $\begin{array}{c}\text { All Grades } \\
(\mathbf{G r} \mathbf{1 - 3})\end{array}$ & Grade 1 & Grade 2 & Grade 3 \\
\hline $\begin{array}{c}\text { Concentration } \\
\text { Impairment }\end{array}$ & $14(82)$ & $9(53)$ & $4(24)$ & $1(6)$ \\
\hline Fatigue & $11(65)$ & $3(18)$ & $8(47)$ & $0(0)$ \\
\hline Tremor & $10(59)$ & $9(53)$ & $1(6)$ & $0(0)$ \\
\hline Nausea & $9(53)$ & $7(41)$ & $0(0)$ & $2(12)$ \\
\hline Dizziness & $6(35)$ & $3(18)$ & $1(6)$ & $2(12)$ \\
\hline Blurred Vision & $4(24)$ & $3(18)$ & $1(6)$ & $0(0)$ \\
\hline Hypotension & $4(24)$ & $1(6)$ & $2(12)$ & $1(6)$ \\
\hline Vomiting & $3(18)$ & $3(18)$ & $0(0)$ & $0(0)$ \\
\hline Edema (limbs) & $3(18)$ & $2(12)$ & $1(6)$ & $0(0)$ \\
\hline Fall & $3(18)$ & $1(6)$ & $1(6)$ & $1(6)$ \\
\hline Gait Disturbance & $3(18)$ & $3(18)$ & $0(0)$ & $0(0)$ \\
\hline Malaise & $3(18)$ & $0(0)$ & $3(18)$ & $0(0)$ \\
\hline Presyncope & $3(18)$ & $0(0)$ & $3(18)$ & $0(0)$ \\
\hline
\end{tabular}


Table 3. Pharmacokinetics of First Dose (Cycle 1) vs Steady-State (Cycle 2).

\begin{tabular}{|c|c|c|c|c|}
\hline & \multicolumn{2}{|c|}{$600 \mathrm{mg}^{\mathrm{a}}$} & \multicolumn{2}{|c|}{$750 \mathrm{mg}^{\mathrm{b}}$} \\
\hline & $\begin{array}{l}\text { C1D1 }(n=4) \\
\text { Mean } \pm \text { SD }\end{array}$ & $\begin{array}{l}\text { C2D1 }(n=4) \\
\text { Mean } \pm \text { SD }\end{array}$ & $\begin{array}{l}\text { C1D1 }(n=3) \\
\text { Mean } \pm \text { SD }\end{array}$ & $\begin{array}{l}\text { C2D2 }(n=1) \\
\text { Mean } \pm \text { SD }\end{array}$ \\
\hline $\mathbf{C}_{\text {MAX }}{ }^{\mathbf{c}}(\mathrm{mg} / \mathrm{L})$ & $4.07 \pm 1.92$ & $4.66 \pm 1.16$ & $5.11 \pm 1.9$ & 5.65 \\
\hline $\begin{array}{l}\mathbf{C}_{\text {MAX }} / \mathbf{D}^{\mathbf{c}} \\
(\mu \mathrm{g} / \mathrm{L} / \mathrm{mg})\end{array}$ & $6.77 \pm 3.20$ & $7.76 \pm 1.93$ & $6.82 \pm 2.54$ & 7.53 \\
\hline $\mathbf{T}_{\text {MAX }}(\mathrm{hr})$ & $2.0 \pm 0.0$ & $5.00 \pm 2.58$ & $2.0 \pm 1.7$ & 2.0 \\
\hline $\begin{array}{l}\text { AUC } \\
\left(\mathrm{hr}{ }^{*} \mathrm{mg} / \mathrm{L}\right)\end{array}$ & $33.0 \pm 4.33$ & $55.4 \pm 5.96$ & $44.2 \pm 2.15$ & 63.5 \\
\hline $\begin{array}{l}\text { AUC/D' } \\
\left(\mathrm{hr}{ }^{*} \mu \mathrm{g} / \mathrm{L} / \mathrm{mg}\right)\end{array}$ & $55.1 \pm 7.21$ & $92.3 \pm 9.94$ & $58.9 \pm 2.87$ & 84.6 \\
\hline $\mathbf{T}_{1 / 2}(\mathrm{hr})$ & $16.4 \pm 4.78$ & $14.8 \pm 5.94$ & $10.5 \pm 4.35$ & 18.3 \\
\hline $\mathbf{C L} / \mathbf{F}^{\mathbf{d}}(\mathrm{L} / \mathrm{hr})$ & $18.4 \pm 2.50$ & $10.9 \pm 1.32$ & $17.0 \pm 0.84$ & 11.8 \\
\hline $\mathbf{V z / F}(\mathrm{L})$ & $438 \pm 146$ & $229 \pm 77.7$ & $260 \pm 121$ & 312 \\
\hline
\end{tabular}

Abbreviations: AUC = area under plasma concentration versus time curve (extrapolated to infinity for first dose, $\mathrm{AUC}_{\mathrm{TAU}}$ for SS); $\mathrm{AUC}_{\mathrm{INF}}=\mathrm{AUC}$ extrapolated to time infinity; $\mathrm{C} 1 \mathrm{D} 1=$ cycle 1 day 1 ; $\mathrm{C} 2 \mathrm{D} 1=$ cycle 2 day $1 ; \mathrm{C} 2 \mathrm{D} 2=$ cycle 2 day $2 ; \mathrm{CL} / \mathrm{F}=$ apparent oral clearance; $\mathrm{C}_{\mathrm{MAX}}=$ maximum plasma concentration; $\mathrm{D}=$ dose $\mathrm{SS}=$ steady state; $\mathrm{t}_{1 / 2}=$ half-life; $\mathrm{T}_{\mathrm{MAX}}=$ time to $\mathrm{C}_{\mathrm{MAX}} ; \mathrm{Vz} / \mathrm{F}=$ apparent oral volume of distribution in terminal phase (determined based on $\mathrm{CL} / \mathrm{F}$ method used, i.e. $\mathrm{Vz} / \mathrm{F}=\mathrm{CL} / \mathrm{F} / \mathrm{k}_{\mathrm{EL}}$ )

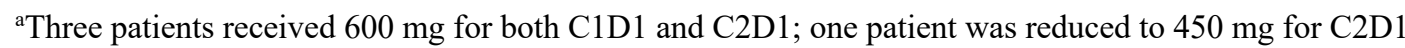
(data not included here)

${ }^{\mathrm{b}}$ Only one patient with PK data received $750 \mathrm{mg}$ for both C1D1 and C2D1; one patient was reduced to $600 \mathrm{mg}$ for C2D1 and another did not have C2D1 data available.

${ }^{\mathrm{c} D o s e}$-normalized parameters

${ }^{\mathrm{d}} \mathrm{CL} / \mathrm{F}$ for first dose calculated as Dose/AUC INF; CL/F for SS calculated as Dose/AUC $\mathrm{TAU}_{\mathrm{TAU}}$ 


\section{Supplemental Methods.}

\section{Noncompartmental Pharmacokinetic Analysis}

First dose PK parameters were calculated using noncompartmental methods (Phoenix WinNonlin 7.0, Certara Pharsight Corp, Cary, NC). Any plasma concentration measured below the LLOQ was excluded from analyses. The maximum plasma concentration $\left(\mathrm{C}_{\mathrm{MAX}}\right)$ and time to $\mathrm{C}_{\mathrm{MAX}}\left(\mathrm{T}_{\mathrm{MAX}}\right)$ were recorded as observed values. The area under the plasma concentration vs time curve to the last observed time point (AUC $\mathrm{LAST}_{\text {) }}$ was calculated using the Linear Up Log Down trapezoidal rule. The elimination rate $\left(\mathrm{k}_{\mathrm{EL}}\right)$ was calculated as the slope of the log-transformed concentrations vs terminal time points. AUC extrapolated to time infinity $\left(\mathrm{AUC}_{\mathrm{INF}}\right)$ was calculated as $\mathrm{AUC}_{\mathrm{LAST}}+\mathrm{C}_{\mathrm{LAST}} / \mathrm{k}_{\mathrm{EL}}$, where $\mathrm{C}_{\mathrm{LAST}}$ is the concentration at the last observed time point. Half-life $\left(\mathrm{t}_{1 / 2}\right)$ was calculated as $\ln (2) / \mathrm{k}_{\mathrm{EL}}$. Apparent oral clearance $(\mathrm{CL} / \mathrm{F})$ was calculated as dose/AUC $\mathrm{INF}_{\mathrm{IN}}$; apparent oral volume of distribution $(\mathrm{Vz} / \mathrm{F})$ was calculated as $\mathrm{CL} / \mathrm{F}$ divided by $\mathrm{k}_{\mathrm{EL}}$. PK parameters at steady-state (Cycle 2 Day 1) were calculated the same except AUC was calculated over the dosing interval tau $=24 \mathrm{hr}\left(\mathrm{AUC}_{\mathrm{TAU}}\right)$, clearance $(\mathrm{CLss} / \mathrm{F})$ as dose/AUC $\mathrm{TAU}_{\mathrm{T}}$, and $\mathrm{Vz} / \mathrm{F}$ as $\mathrm{CLss} / \mathrm{F}$ divided by $\mathrm{k}_{\mathrm{EL}}$. The number of subjects included in the pharmacokinetic analysis was insufficient for propor statistical testing. 
Supplemental Table 1. Adverse Events with an Attribution of at Least Possible Occurring in Greater than $15 \%$ of Patients Who Received Study Treatment $(\mathrm{N}=17)$ Sorted by Dosing Strategy

\begin{tabular}{|c|c|c|c|c|c|c|}
\cline { 2 - 7 } \multicolumn{1}{c|}{} & \multicolumn{2}{c|}{$\begin{array}{c}\mathbf{4 5 0} \text { mg Twice } \\
\text { Daily } \\
\text { N= }\end{array}$} & \multicolumn{2}{c|}{$\begin{array}{c}\mathbf{7 5 0 ~ m g ~ O n c e ~} \\
\text { Daily } \\
\text { N= }\end{array}$} & \multicolumn{2}{c|}{$\begin{array}{c}\text { 600 mg Once Daily } \\
\mathbf{N}=\mathbf{4}\end{array}$} \\
\hline Adverse Event & $\begin{array}{c}\text { Grade } \\
\mathbf{1 / 2}\end{array}$ & $\begin{array}{c}\text { Grade } \\
\mathbf{3}\end{array}$ & $\begin{array}{c}\text { Grade } \\
\mathbf{1} / \mathbf{2}\end{array}$ & $\begin{array}{c}\text { Grade } \\
\mathbf{3}\end{array}$ & $\begin{array}{c}\text { Grade } \\
\mathbf{1} / \mathbf{2}\end{array}$ & Grade 3 \\
\hline $\begin{array}{c}\text { Concentration } \\
\text { Impairment }\end{array}$ & $6(86)$ & $0(0)$ & $5(83)$ & $1(17)$ & $2(50)$ & $0(0)$ \\
\hline Fatigue & $5(71)$ & $0(0)$ & $4(67)$ & $0(0)$ & $2(50)$ & $0(0)$ \\
\hline Tremor & $3(43)$ & $0(0)$ & $5(83)$ & $0(0)$ & $2(50)$ & $0(0)$ \\
\hline Nausea & $4(57)$ & $1(14)$ & $0(0)$ & $0(0)$ & $3(75)$ & $1(25)$ \\
\hline Dizziness & $2(29)$ & $1(14)$ & $1(17)$ & $1(17)$ & $1(25)$ & $0(0)$ \\
\hline Blurred Vision & $1(14)$ & $0(0)$ & $1(17)$ & $0(0)$ & $2(50)$ & $0(0)$ \\
\hline Hypotension & $3(43)$ & $0(0)$ & $0(0)$ & $0(0)$ & $0(0)$ & $1(25)$ \\
\hline Vomiting & $1(14)$ & $0(0)$ & $0(0)$ & $0(0)$ & $2(50)$ & $0(0)$ \\
\hline Edema (limbs) & $1(14)$ & $0(0)$ & $1(17)$ & $0(0)$ & $1(25)$ & $0(0)$ \\
\hline Fall & $1(14)$ & $1(14)$ & $0(0)$ & $0(0)$ & $1(25)$ & $0(0)$ \\
\hline Gait Disturbance & $3(43)$ & $0(0)$ & $0(0)$ & $0(0)$ & $0(0)$ & $0(0)$ \\
\hline Malaise & $1(14)$ & $0(0)$ & $2(33)$ & $0(0)$ & $0(0)$ & $0(0)$ \\
\hline Presyncope & $3(43)$ & $0(0)$ & $0(0)$ & $0(0)$ & $0(0)$ & $0(0)$ \\
\hline
\end{tabular}




\section{Chapter 4}

\section{Anti-tumor Activity of NLG207 (formerly CRLX101) in Combination with Enzalutamide in Preclinical Prostate Cancer Models}

Keith T. Schmidt, ${ }^{1}$ Cindy H. Chau ${ }^{1}$, Jonathan D. Strope ${ }^{1}$, Alwin D. R. Huitema, ${ }^{2,3}$

Tristan M. Sissung ${ }^{1}$, Douglas K. Price ${ }^{1}$, and William D. Figg ${ }^{1}$

${ }^{1}$ Genitourinary Malignancies Branch, Center for Cancer Research, National Cancer Institute, National Institutes of Health, Bethesda, MD, USA

${ }^{2}$ Dept. Pharmacy \& Pharmacology, Netherlands Cancer Institute, Amsterdam, The Netherlands

${ }^{3}$ Department of Clinical Pharmacy, University Medical Center Utrecht, Utrecht University, Utrecht, The Netherlands

\section{Submitted}




\section{ABSTRACT}

Introduction - Effective treatments for patients with mCRPC following disease progression on enzalutamide is currently an unmet clinical need. Simultaneous inhibition of HIF-1 $\alpha$ and AR pathways has been previously shown to overcome enzalutamide resistance in vitro. Combination treatment with NLG207, a nanoparticledrug conjugate of camptothecin and inhibitor of HIF-1 $\alpha$, and enzalutamide was evaluated in preclinical prostate cancer models of enzalutamide resistance.

Methods - The effect of NLG207 and enzalutamide on average tumor volume and tumor re-growth after 3 weeks of treatment was evaluated in vivo using the subcutaneous 22Rv1 xenograft and castrated subcutaneous VCaP xenograft models. Correlative assessments of anti-tumor activity were evaluated in vitro using cell proliferation and qPCR assays.

Results - NLG207 in combination with enzalutamide reduced average tumor volume by $93 \%$ after 3 weeks of treatment $(p<0.05)$ and reduced the median rate of tumor growth by $54 \%(\mathrm{p}<0.0001)$ in comparison to vehicle control in the subcutaneous $22 \mathrm{Rv} 1$ xenograft model. The addition of NLG207 also enhanced the efficacy of enzalutamide alone in the castrated subcutaneous $\mathrm{VCaP}$ xenograft model, decreasing the median rate of tumor growth by $51 \%(\mathrm{p}=0.0001)$. In vitro assessments of cell proliferation and gene expression further demonstrated anti-tumor activity via AR-HIF-1 $\alpha$ crosstalk inhibition.

Conclusions - Combination treatment with NLG207 and enzalutamide was shown to be effective in preclinical prostate cancer models of enzalutamide resistance. Clinical investigation of this treatment combination is ongoing (NCT03531827). 


\section{INTRODUCTION}

Enzalutamide, an androgen receptor antagonist (ARA), and abiraterone acetate, a CYP17 inhibitor, are current primary standard of care treatment options for patients with metastatic castration-resistant prostate cancer (mCRPC). ${ }^{1}$ Acquired resistance to these agents is inevitable, ultimately resulting in clinical disease progression and initiation of additional lines of therapy. ${ }^{2}$ Mechanisms of acquired resistance include alterations of DNA damage repair mechanisms, activation of alternative cell signaling pathways (e.g. $\mathrm{PI} 3 \mathrm{~K}, \mathrm{Wnt} / \beta$-catenin), and aberrations in androgen receptor (AR) activity. ${ }^{3}$ Notably, AR-full length (AR-FL) overexpression and expression of AR splice variants (e.g. ARV7) are commonly associated with disease progression following enzalutamide monotherapy. ${ }^{4-7}$ Recent clinical investigations often focus on combinatorial treatment approaches aimed to target one or multiple pathways of acquired resistance.

Intratumoral hypoxia serves an important role in prostate cancer aggressiveness and metastatic potential, as cells adapt to hypoxic environments via co-opting blood vessel formation and migrating towards vessels. ${ }^{8-11}$ Hypoxia-Inducible Factor (HIF)-1 $\alpha$, a transcription factor upregulated in response to hypoxia, is responsible for promoting tumor angiogenesis, anaerobic metabolism, immunity, adaptation, and invasion. ${ }^{11-13}$ Androgen deprivation can also contribute to prostate cancer cell adaptation to hypoxic environments, upregulating transcriptional activity of the AR. ${ }^{14,15}$ Crosstalk between the HIF-1 $\alpha$ and AR pathways has been suggested via a ternary complex comprising AR, HIF- $1 \alpha$ and $\beta$-catenin on androgen response elements of AR target genes. ${ }^{16-18}$ Thus, targeting HIF-1 $\alpha$ was hypothesized to down-regulate AR-mediated gene expression and 
reduce prostate cancer cell proliferation. Our laboratory previously investigated the dual targeting of both axes via combination treatment of enzalutamide with HIF-1 $\alpha$ inhibition in prostate cancer cells to define the molecular mechanisms by which HIF-1 $\alpha$ inhibition potentiates anti-AR therapy in CRPC. HIF-1 $\alpha$ inhibition, achieved via chetomin (disruptor of HIF-1 $\alpha$-p300 interactions) or siRNA silencing of HIF-1 $\alpha$, in combination with enzalutamide synergistically reduced AR-regulated and HIF-1 $\alpha$ mediated transcription, reduced VEGF protein expression, and inhibited cell growth. ${ }^{18}$ In 22Rv1 cells, a cell line with enzalutamide resistance mediated via androgen independence and significant AR splice variant expression, ${ }^{19-21}$ enzalutamide activity was significantly enhanced following HIF-1 $\alpha$ inhibition. ${ }^{18}$

In the current study, we further investigate this therapeutic approach by evaluating the combination treatment of enzalutamide with NLG207, formerly known as CRLX101. NLG207 is a nanoparticle-drug conjugate of camptothecin designed to overcome the poor physicochemical properties associated with small molecule camptothecin, while also utilizing the enhanced permeation and retention (EPR) effect to more optimally facilitate drug delivery to tumors. ${ }^{22,23}$ Camptothecins, potent inhibitors of topoisomerase I, have previously been shown to block the accumulation of HIF- $1 \alpha$ and subsequently the expression of VEGF. ${ }^{24,25}$ Studies with NLG207 have also demonstrated effective targeting of HIF-1 $\alpha$ and inhibition of angiogenesis in models of ovarian cancer, breast cancer and glioblastoma, either as monotherapy or in combination with bevacizumab. ${ }^{26-29}$ Thus, we assessed the anti-tumor activity of NLG207 in 
combination with enzalutamide in subcutaneous xenograft models of prostate cancer with clinically relevant mechanisms of acquired resistance to enzalutamide.

\section{METHODS}

\section{Cell Culture}

22Rv1 cells were maintained in phenol red-free RPMI 1640, and VCaP cells were maintained in DMEM, both supplemented with 10\% fetal bovine serum (FBS), 50 $\mathrm{U} / \mathrm{mL}$ Penicillin, and $50 \mathrm{mg} / \mathrm{mL}$ Streptomycin. Both 22Rv1 and VCaP cells were purchased from American Type Culture Collection (ATCC; Manassas, VA). ATCC uses Short Tandem Repeat (STR) profiling for testing and authentication of cell lines.

\section{$\underline{\text { Reagents }}$}

R1881 and S-(+)-Camptothecin were purchased from Millipore Sigma (Rockville, MD), enzalutamide was purchased from Selleck Chemicals (Houston, TX), and NLG207 was provided by NewLink Genetics (Ames, IA). All doses of NLG207 (mg) were measured using camptothecin (CPT) equivalents, or the mass of CPT contained within the nanoparticle formulation.

\section{Cell Proliferation Assays}

22Rv1 and VCaP cells were seeded in 96-well plates in $100 \mu \mathrm{L} 10 \%$ CharcoalDextran Stripped (CDS) FBS supplemented phenol-red free RPMI 1640 and DMEM (supplemented with $4 \mathrm{mM} \mathrm{L-gluatmine)} \mathrm{medium,} \mathrm{respectively.} \mathrm{Cells} \mathrm{were} \mathrm{seeded} \mathrm{at}$ densities of 5,000 and 30,000 cells per well for 22Rv1 and VCaP, respectively. Following 24-48 hour incubation, cells were treated with the corresponding 10\% CDS- 
FBS supplemented media containing $0.1 \mathrm{nM} \mathrm{R} 1881$ and either DMSO control, CPT, NLG207, enzalutamide (ENZ), CPT + ENZ, or NLG207 + ENZ (day 0). Cells were redosed on day 4 post-treatment. Cell viability was measured on days $0,3,5$, and 7 using the Cell Counting Kit-8 cell viability assay according to the manufacturer's instructions (Dojindo, Rockville, MD), and absorbance was read at $450 \mathrm{~nm}$ using a SpectraMax iD3 fluorescence plate reader (Molecular Devices, Sunnyvale, CA).

\section{Semiquantitative Real-Time Polymerase Chain Reaction}

VCaP cells were plated at a density of 800,000 cells per well in a 6-well dish in phenol red-free DMEM media supplemented with 10\% CDS-FBS for 48 hours. Cells were then treated with $10 \%$ CDS-FBS supplemented media with or without $0.1 \mathrm{nM}$ R1881 and combinations of DMSO control, 500 nM CPT, 500 nM NLG207, and/or 500 nM ENZ for 24 hours. Total RNA was extracted using the RNAeasy mini kit (Qiagen, Germantown, MD) per manufacturer's protocol. Purified RNA $(\sim 0.24 \mu \mathrm{g})$ was reverse transcribed via $30 \mu \mathrm{L}$ cDNA synthesis reaction using the SuperScript III First Strand Synthesis System (Invitrogen, Carlsbad, CA) per manufacturer's protocol. cDNA synthesis products were amplified in triplicate with forward and reverse primers: KLK3 (Hs02576345_m1, Applied Biosystems, Foster City, CA), ERG (Hs01554629_m1, Applied Biosystems), VEGFA (Hs00900055_m1, Applied Biosystems), LDHA (Hs00855332_g1, Applied Biosystems), and ACTB (Hs99999903_m1, Applied Biosystems). Custom primers (Applied Biosystems) for ARFL and AR-V7, previously described by Markowski et al., were also used to amplify cDNA. ${ }^{30}$ The specificity of these primers were evaluated in PC3, 22Rv1, and VCaP 
cells, and compared to LNCaP95 cells. All other primers used were commercially available and previously validated. ${ }^{18,31}$

Two microliters of cDNA per sample was mixed with $1 \mu \mathrm{L}$ forward and reverse primers, $7 \mu \mathrm{L}$ water, and $10 \mu \mathrm{L}$ Taqman Gene Expression Master Mix (Applied Biosystems) for a total of $20 \mu \mathrm{L}$. Semiquantitative real-time polymerase chain reaction (qPCR) was performed using an Applied Biosystems StepOnePlus Real-Time PCR system with StepOne Software. All qPCR reactions were run in triplicate using the standard Taqman protocol for 40 cycles, with use of $\beta$-actin (ACTB) as the reference housekeeping gene. Fold-change in RNA levels was calculated using the $\Delta \Delta \mathrm{C}_{\mathrm{t}}$ method. For AR-FL and AR-V7 primer validation, the same qPCR reaction conditions were used for 30 cycles. PCR products were mixed with DNA gel loading dye (6x; Thermo Fisher Scientific, Waltham, MA), applied to 4-20\% TBE gels (Invitrogen) and stained with SYBR Green (Invitrogen). The GeneRuler 50 bp DNA Ladder (Thermo Fisher Scientific) was used as a size standard, and PCR fragments were visualized using an Odyssey Fc Imager (LI-COR Biosciences, Lincoln, NE) (Figure S1).

\section{Animal Care}

All animals were housed in a pathogen-free facility of the National Cancer Institute, which is accredited by the Association for Assessment and Accreditation of Laboratory Animal Care (AAALAC) International and follows the Public Health Service (PHS) Policy for the Care and Use of Laboratory Animals. Animal care was provided in accordance with the Guide for the Care and Use of Laboratory Animals. The study protocol was approved by the NCI Animal Care and Use Committee (ACUC). 
Approximately $5 \times 10^{6} 22 \mathrm{Rv} 1$ cells (suspended in DPBS) were subcutaneously injected into the rear flank of six-week old, male, severe combined immunodeficiency (SCID) mice. Tumors were grown to a volume greater than $50 \mathrm{~mm}^{3}$ before stratification into treatment groups (Study 1:n=4-5; Study 2: $n=9-10)$ : vehicle control, ENZ only (25 $\mathrm{mg} / \mathrm{kg}$ in 50:50 PEG-400:Tween 80 via daily oral gavage), NLG207 4 or $8 \mathrm{mg} / \mathrm{kg}$ (in DPBS via weekly i.p. injection) \pm ENZ. Mice were treated for 3 weeks, with weight measurements daily and tumor volume measurements three times weekly, using the formula $\mathrm{V}=\left(\mathrm{L}^{*} \mathrm{~W}^{2}\right) / 2$ (length corresponding to longer dimension of tumor). Following 3 weeks of treatment in the first study, the mice were euthanized and tumors were harvested to obtain final weight and volume (using $\left.\mathrm{L}^{*} \mathrm{~W} * \mathrm{H}^{*}(\pi / 6)\right)$ measurements. In the second study, mice were followed after treatment course completion three times weekly until tumors ulcerated or reached $>2 \mathrm{~cm}$ in one direction, prompting removal from study and euthanasia per NCI ACUC guidance; mice with $>20 \%$ reduction in body weight were removed from study and censored.

\section{Castrated VCaP Subcutaneous Xenograft}

Approximately $2.5 \times 10^{6} \mathrm{VCaP}$ cells (suspended in 50:50 DPBS:Matrigel) were injected into the rear flank of six-week old, male, SCID mice. When the average tumor volume reached $\sim 200 \mathrm{~mm}^{3}$ (measured via $\mathrm{V}=\left(\mathrm{L}^{*} \mathrm{~W}^{2}\right) / 2$ ) the mice were castrated (removal of the testes facilitated via scrotal incision and vaginal tunic access) under isoflurane anesthesia. The mice were followed up post-surgically for 10 days, and tumors were allowed to regrow to an average volume of $\sim 200 \mathrm{~mm}^{3}$. The mice were then 
stratified on the basis of tumor volume into 4 treatment groups ( $n=9-10)$ : vehicle control, ENZ $25 \mathrm{mg} / \mathrm{kg}$ daily, NLG207 $8 \mathrm{mg} / \mathrm{kg}$ once weekly, and combination. Mice were treated for 3 weeks, with tumor measurements obtained 3 times weekly and body weight measurements collected daily. Following 3 weeks of treatment, animals were monitored for tumor size and body weight three times weekly. Mice were removed from study if tumors reached $>2 \mathrm{~cm}$ in any dimension per NCI ACUC guidance; mice with $>20 \%$ reduction in body weight were removed from study and censored. Following 6 weeks post treatment, all remaining animals were euthanized.

\section{$\underline{\text { Statistical Analyses }}$}

Unpaired $t$ tests were used for between group comparisons of cell proliferation. Tumor growth curves were reported as mean tumor volumes \pm SEM and mean percentage change in tumor volume (start of treatment as baseline) \pm SEM for the 22Rv1 and VCaP xenograft models, respectively. Body weight curves were reported as mean body weight \pm SEM. Comparisons of average tumor volumes, average tumor volume change, average tumor weights, and fold change in gene expression at specified timepoints were made using Mann-Whitney tests. Kaplan-Meier method was used to assess median survival (i.e. time to tumor $>2 \mathrm{~cm}$ in one dimension) and progression-free survival (i.e. tumor doubling in size from baseline) for the 22Rv1 and $\mathrm{VCaP}$ xenograft models, respectively. Log-rank (Mantel-Cox) tests were used to determine statistical significance of survival differences between treatment groups. Statistical analyses were performed using GraphPad Prism 8 ( $\mathrm{p}<0.05$ was used as the threshold for statistical significance). 


\section{RESULTS}

Enhanced Activity of Enzalutamide in Combination with NLG207 in 22Rv1 Cells

First, the effect of NLG207 and enzalutamide on the growth of 22Rv1 cells was evaluated in vitro. The calculated $\mathrm{IC}_{50}$ values for NLG207 and CPT in 22Rv1 cells were $10 \mathrm{nM}$ and $5 \mathrm{nM}$, respectively; CPT was included to confirm activity was associated with the CPT component of NLG207. The $\mathrm{IC}_{50}$ value for enzalutamide in $22 \mathrm{Rv} 1$ cells was $1 \mu \mathrm{M}$, consistent with previous literature. ${ }^{21}$ ENZ, NLG207 and CPT each significantly down-regulated cell proliferation in the presence of $0.1 \mathrm{nM}$ R 1881 by 5 days of treatment (Figure 1A and 1B). Treatment with NLG207 or CPT in combination with ENZ in $22 \mathrm{Rv} 1$ cells enhanced the effect of ENZ alone by $58.3 \%$ and $59.9 \%$ by day 7 post-treatment initiation, respectively $(\mathrm{p}<0.0001)$. The enhancement of enzalutamide anti-tumor activity via NLG207 co-treatment mirrored previous data with siRNA inhibition of HIF + ENZ in 22Rv1 cells. ${ }^{18}$

\section{Efficacy of NLG207 and enzalutamide in 22Rv1 xenograft model}

The efficacy of NLG207 and ENZ was first evaluated via analysis of tumor volume reduction following 3 weeks of treatment using the subcutaneous $22 \mathrm{Rv} 1$ xenograft model, a model previously used by Liu et al. ${ }^{32,33}$ The $4 \mathrm{mg} / \mathrm{kg}$ and $8 \mathrm{mg} / \mathrm{kg}$ doses of NLG207 were derived from prior xenograft studies by Pham et al. ${ }^{28,29}$ Comparisons of daily tumor measurements collected on study showed NLG207 $8 \mathrm{mg} / \mathrm{kg}$ $\pm \mathrm{ENZ}$ and NLG207 $4 \mathrm{mg} / \mathrm{kg} \pm \mathrm{ENZ}$ significantly reduced average tumor volume compared to vehicle control by day 7 and day 11 post-treatment, respectively $(p<0.05)$ (Figure 2A). All groups treated with NLG207 had significant reductions in tumor 
volume compared to ENZ alone by day $14(\mathrm{p}<0.05)$. In harvested tumors, average volume and weight comparisons between vehicle control and ENZ alone were consistent with enzalutamide resistance ( $>>0.05$; Figure 2B, 2C, and 2D). All NLG207 treated groups had significantly reduced average harvested tumor weights compared to either vehicle control or ENZ alone $(\mathrm{p}<0.05)$. Relative to vehicle control, treatment with NLG207 $8 \mathrm{mg} / \mathrm{kg}+$ ENZ reduced harvested tumor volume by 93\%, and NLG207 4 $\mathrm{mg} / \mathrm{kg}+$ ENZ, by comparison, only showed an $81 \%$ reduction $(\mathrm{p}<0.05)$. Based on this data, we used the $8 \mathrm{mg} / \mathrm{kg}$ dose of NLG207 for future xenograft studies.

We conducted an additional subcutaneous 22Rv1 xenograft study of NLG207 with or without ENZ treatment to assess tumor re-growth following 3 weeks of treatment, an endpoint previously used to evaluate NLG207 efficacy. ${ }^{34}$ NLG207 \pm ENZ significantly reduced average tumor volume compared to vehicle control \pm ENZ by day $7(\mathrm{p}<0.05$, Figure 3A). Median survival of mice treated with NLG207 or NLG207+ ENZ (73.5 and 81 days, respectively) was approximately doubled in comparison to mice treated with either ENZ or vehicle control (37 days for both groups), as shown in Figure 3B $(p<0.0001)$. Taken together, results from the 22Rv1 xenograft model confirmed enzalutamide resistance, and demonstrated the efficacy of both NLG207 alone and NLG207 in combination with enzalutamide.

Toxicity was evaluated via average body weight measurements, taken daily during treatment and three times weekly post-treatment (Figures $2 \mathbf{E}$ and $\mathbf{3 C}$ ). A total of 3 mice were removed from both studies due to $>20 \%$ reduction in body weight: one mouse receiving NLG207 $8 \mathrm{mg} / \mathrm{kg}$ in the first study, and one mouse receiving NLG207 
$8 \mathrm{mg} / \mathrm{kg}+\mathrm{ENZ}$ from both the first study and the second study. Trends of decreased average body weight were seen in groups receiving NLG207 compared to either vehicle control or ENZ alone on both studies.

\section{Effect of NLG207 on cell proliferation and gene expression in VCaP cells}

The anti-tumor activity of NLG207 + ENZ was further evaluated in the VCaP cell line. Unlike 22Rv1 cells, which express AR-V7 and other splice variants via AR intragenic rearrangement, $\mathrm{VCaP}$ cells express $\mathrm{AR}-\mathrm{V} 7$ via significantly increased $\mathrm{AR}$ gene transcript generation. ${ }^{19,20,32,35} \mathrm{VCaP}$ cells also overexpress AR-FL and harbor the TMPRSS2-ERG fusion, relevant characteristics in enzalutamide-resistant mCRPC that are not present in $22 \mathrm{Rv} 1$ cells. ${ }^{35}$ The in vitro $\mathrm{IC}_{50}$ values were $500 \mathrm{nM}$ for NLG207 and $500 \mathrm{nM}$ for $\mathrm{CPT}$ in $\mathrm{VCaP}$ cells, a 50-fold and 100-fold decrease in potency, respectively, compared to $22 \mathrm{Rv} 1$ cells. In $\mathrm{VCaP}$ cells, the $\mathrm{IC}_{50}$ value for $\mathrm{ENZ}$ was 500 $\mathrm{nM}$, a two-fold increase in potency compared to $22 \mathrm{Rv} 1$ and similar to the previously reported value. ${ }^{36}$ ENZ, NLG207 and CPT each significantly down-regulated cell proliferation in the presence of $0.1 \mathrm{nM} \mathrm{R} 1881$ by day 3 post-treatment (Figure $4 \mathrm{~A}$ and 4B). Treatment of NLG207 or CPT in combination with ENZ enhanced cell growth inhibition of ENZ alone by $60 \%$ and $61.5 \%$, respectively, by day $7(\mathrm{p}<0.0001)$.

We next examined the effect of drug treatments on changes in mRNA expression of AR pathway genes in VCaP cells treated with $0.1 \mathrm{nM} \mathrm{R} 1881$ in vitro. Trends in ARassociated gene expression in response to androgen stimulation with or without enzalutamide (Figure 4) was consistent with prior literature..$^{35,37-39}$ Androgen stimulation alone or with NLG207 treatment preferentially suppressed AR-V7 vs. AR- 
FL mRNA expression while this effect is blunted following ENZ treatment (Fig. 4C \& 4D). Treatment with $500 \mathrm{nM}$ NLG207 alone had a more robust effect on AR-FL/AR-V7 compared to androgen stimulation. The addition of NLG207 to ENZ treatment more effectively attenuated AR-V7 expression (2.9-fold compared to ENZ alone, $\mathrm{p}<0.0001$ ).

Downstream AR target genes, KLK3 and ERG, were significantly downregulated following combination treatment, with 2.8-fold and 4.6-fold reductions observed compared to ENZ alone, respectively (Fig. 4E \& 4F; $<<0.0001)$. Treatment with 500 nM CPT instead of NLG207 resulted in similar but more robust changes in gene expression, likely explained via nanoparticle release kinetics (Fig. S2). VEGFA and LDHA, HIF-1 $\alpha$ downstream target genes, were also down-regulated following treatment with NLG207 or CPT (Fig. S3); combination CPT + ENZ treatment resulted in 1.7-fold and 2.5-fold reductions in VEGFA and LDHA, respectively, when compared to ENZ alone $(\mathrm{p}<0.0001)$. Similar findings were noted in our prior study examining the effect of combination chetomin and enzalutamide on target gene expression. ${ }^{18}$

\section{NLG207 Enhances Enzalutamide Activity in Castrated VCaP Xenografted Mice}

Finally, we investigated the efficacy of NLG207 $8 \mathrm{mg} / \mathrm{kg}+$ ENZ $25 \mathrm{mg} / \mathrm{kg}$ in the castrated VCaP subcutaneous xenograft model. Cai et al. first described the effects of castration on pre-established $\mathrm{VCaP}$ xenografted tumors, demonstrating that AR activity and ERG expression are restored following castration, with marked increases in AR mRNA expression. ${ }^{40}$ Evaluation of NLG207 and enzalutamide anti-tumor activity in the castrated VCaP subcutaneous xenograft model was implemented similarly to recent preclinical evaluations of second-generation AR antagonist-based treatments. ${ }^{36,41}$ The 
average percent change in tumor volume from baseline per treatment group is summarized in Figure 5A. The NLG207 treated groups had significant reductions in tumor volume compared to vehicle control or ENZ by day 10 post-treatment initiation $(p<0.05)$. NLG207 + ENZ was significantly better than NLG207 alone $(p \leq 0.05)$ throughout several timepoints post-treatment initiation, including day 8 (post-second NLG207 injection), day 22 (end of treatment [EOT]), and day 36 (two weeks postEOT). While the effect of NLG207 with enzalutamide provided a persistent reduction in tumor growth in comparison to NLG207 alone well after treatment cessation, the effect of enzalutamide in comparison to vehicle control dissipated prior to the end of treatment. The median progression-free survival (i.e tumor doubling time) was different between the vehicle control and ENZ alone groups (Figure 5B), both reached prior to EOT (13 and 20 days, respectively; $<<0.05$ ); the anti-tumor effect of ENZ alone was consistent with prior studies describing minimal sensitivity to the agent in the castrated VCaP xenograft model. ${ }^{36,41}$ The median progression-free survival of the NLG207 8 $\mathrm{mg} / \mathrm{kg}+$ ENZ treatment group was significantly improved in comparison to NLG207 8 $\mathrm{mg} / \mathrm{kg}$ alone (31 and 41 days, respectively, $\mathrm{p}<0.05$ ). Importantly, the addition of NLG207 to ENZ treatment reduced the median rate of tumor growth (assessed via progression-free survival) by $51 \%$ in comparison to $\mathrm{ENZ}$ alone $(\mathrm{p}=0.0001)$.

Castration and NLG207 treatment were associated with declines in average body weight measurements (Figure 5C). A brief reduction in average body weight was observed during post-castration follow-up, with weights stabilizing close to baseline prior to treatment initiation. NLG207 alone was the least well tolerated, with average body weight nadirs occurring 3 days post-i.p. injection; ENZ appeared to negate this 
effect, as NLG207 + ENZ average body weight nadirs were smaller by comparison.

Three mice were euthanized following $>20 \%$ body weight reduction: one during postcastration follow-up, one from the NLG207 alone group (day 7 of treatment) and one from the NLG207 + ENZ group (day 10 of treatment).

\section{DISCUSSION}

The use of NLG207 as a HIF-1 $\alpha$ inhibitor in combination with enzalutamide presented a reasonable approach to down-regulate AR-HIF crosstalk, as suggested by prior study in enzalutamide-resistant 22Rv1 cells. ${ }^{18}$ The addition of NLG207 potentiated the anti-AR effects of ENZ in preclinical models of AR-mediated acquired resistance, most notably in the castrated $\mathrm{VCaP}$ xenograft model, which exhibits both AR amplification and splice variant expression. The combination treatment demonstrated robust activity in both the $22 \mathrm{Rv} 1$ and castrated $\mathrm{VCaP}$ xenograft models, with evidence supporting the downregulation of AR pathway-gene expression, including AR-FL, ARV7 and downstream targets.

NLG207 enhanced enzalutamide's ability to down-regulate the mRNA expression of KLK3 and ERG, both downstream AR-target genes, in VCaP cells, mirroring previous data shown with chetomin and enzalutamide. ${ }^{18}$ Using validated AR species specific primers, ${ }^{30}$ NLG207 and CPT were shown to down-regulate AR-FL and AR-V7 mRNA expression. Downstream targets of HIF-1 $\alpha$ signaling, VEGFA and LDHA, were also down-regulated in response to NLG207 and CPT under normoxia.

The impact of treatment VEGFA and LDHA mRNA expression was not evaluated in the 
context of hypoxia and subsequent HIF-1 $\alpha$ accumulation, a limitation of the present study. ${ }^{18}$ Additionally, we lacked sufficient tumor tissue to compare in vivo gene and protein expression of relevant pharmacodynamic biomarkers post-treatment due to our xenograft study endpoint selection. ${ }^{32,34}$ Given the robust suppression of NLG207 on ARFL/AR-V7, whether NLG207 has an effect on the heterodimerization of AR-FL with AR-V7 remains to be determined; however, preliminary evidence has been suggested by CPT in 22Rv1 cells. ${ }^{42}$

To explain the anti-tumor activity of the treatment combination, we first addressed the role of AR expression with emphasis on AR copy number gain in $\mathrm{VCaP}$ cells. It was hypothesized that NLG207 could globally reduce AR gene transcription, driving down AR-V7 expression responsible for enzalutamide resistance while also aiding enzalutamide activity via reduction of intracellular concentrations of AR-FL. CPT significantly reduced both AR-FL and AR-V7 mRNA expression in the present study, as well as AR mRNA expression in prior studies with LNCaP cells. ${ }^{43,44}$ Selective AR-variant knockdown has been previously shown to restore enzalutamide activity via AR-FL, or androgen-dependent, signaling in $22 \mathrm{Rv} 1$ cells. ${ }^{21}$ With similar levels of ARV7 expression between 22Rv1 and VCaP cells, the significant difference between combination treatment and NLG207 alone observed in the VCaP xenograft model, but not the 22Rv1 xenograft model, may be explained by higher AR-FL concentrations following NLG207 treatment; more complete suppression of AR-FL expression is suggested in 22Rv1 xenografted mice, rendering enzalutamide minimally effective in limited presence of drug target. In a similar study, JQ1, a BET bromodomain inhibitor targeting AR activity, also significantly down-regulated AR-FL and AR-V7 
transcription and enhanced the tumor growth inhibition of enzalutamide in the castrated VCaP xenograft model; ${ }^{41}$ combination NLG207 and enzalutamide reduced tumor volume below baseline measurement, an effect not seen with JQ1 and enzalutamide. ${ }^{41}$ Additionally, when compared to a previous study that assessed niclosamide, an agent that specifically promotes AR-V7 degradation, in the subcutaneous 22Rv1 xenograft model, ${ }^{32}$ the anti-tumor effect of NLG207 appears more potent.

The anti-tumor activity of NLG207 is also facilitated via topoisomerase I inhibition in these preclinical models. The down-regulation of HIF-1 $\alpha$ accumulation following treatment with topotecan, a derivative of camptothecin, was shown to be dependent upon topoisomerase I inhibition. ${ }^{45}$ Treatment with a similar $\beta$-cyclodextrinbased formulation of CPT has previously been shown to both increase phosphorylated H2A.X, a marker of DNA damage induction, and down-regulate AR protein expression. ${ }^{46}$ Evaluation of clinical tumor specimens via immunohistochemistry following NLG207 treatment demonstrated both activity against Ki-67 and downstream targets of HIF-1 $\alpha$ (CAIX, VEGFA) ${ }^{47}$ supporting the multi-faceted nature of anti-tumor activity. NLG207 activity against topoisomerase I thus appears necessary for the observed effects on AR-FL and AR-V7 gene expression and can potentially dominate the anti-tumor effect in cell lines with increased CPT sensitivity. In 22Rv1 xenografted mice, our findings suggested NLG207 $8 \mathrm{mg} / \mathrm{kg}$ provided potent topoisomerase I inhibition, limiting the role of AR-pathway down-regulation and the perceived antitumor effect of ENZ. By contrast, NLG207 treatment facilitated a durable anti-tumor 
effect of enzalutamide in castrated $\mathrm{VCaP}$ xenografted mice, which is best explained by enhanced AR-pathway down-regulation.

The overexpression of ERG, a transcription factor of the ETS family that is promoted via TMPRSS2-ERG fusion protein expression, can increase tumor cell invasiveness, cell proliferation and cellular migration. ${ }^{48} 22 \mathrm{Rv} 1$ cells do not express the TMPRSS2-ERG fusion and have moderately elevated ERG expression, whereas VCaP cells have elevated ERG expression associated with the TMPRSS2-ERG fusion. ${ }^{49}$ Interestingly, a comparison of CPT sensitivity between VCaP and DU-145 cells, which have similar ERG expression to $22 \mathrm{Rv} 1$ cells, ${ }^{49}$ revealed a $>100$-fold difference in potency to $\mathrm{CPT} ;{ }^{50}$ this finding is consistent with the $\mathrm{IC}_{50}$ comparison of $\mathrm{CPT}$ we observed between VCaP and 22Rv1 cells. ERG was also implicated to disrupt the interaction of topoisomerase I and DNA-PKcs, which regulates the cellular response of CPT independently of DNA repair. ${ }^{50}$ Reduced ERG expression in $22 \mathrm{Rv} 1$ cells further suggests that NLG207 $8 \mathrm{mg} / \mathrm{kg}$ alone led to highly potent topoisomerase I inhibition in the 22Rv1 xenograft model. NLG207 and CPT treatment in VCaP cells resulted in significant downregulation of ERG mRNA expression in vitro, which may suggest an "auto-sensitization" effect of CPT in VCaP cells. Though ERG expression in VCaP cells has been implicated to enhance response to enzalutamide, ${ }^{51}$ clinical utility as a predictive biomarker has not been established. Recent data has suggested TMPRSS2ERG to not be a predictive biomarker of enzalutamide efficacy in chemo-naïve patients with mCRPC in the first-line setting. ${ }^{52}$ Future investigations of ERG expression are necessary to better understand CPT sensitivity in prostate cancer cells and the role of 
TMPRSS2-ERG as a predictive biomarker of clinical outcomes following enzalutamide treatment.

With respect to toxicity, weight loss associated with NLG207 treatment has been previously reported in the literature. ${ }^{28}$ Interestingly, NLG207 and ENZ combination therapy appeared to have a weight-sparing effect, most notably in the castrated $\mathrm{VCaP}$ xenograft model. In the $22 \mathrm{Rv} 1$ xenografted mice, a similar weight-sparing effect was also implicated with $4 \mathrm{mg} / \mathrm{kg}$ doses of NLG207, but not the $8 \mathrm{mg} / \mathrm{kg}$ dose. The expression of AR-V7 has been previously shown to restore AR-mediated lipid biosynthesis via study of 22Rv1 and VCaP cells in vitro and in vivo. ${ }^{53}$ Increased AR-V7 mRNA expression following combination treatment in comparison to NLG207 alone, as shown via our in vitro data, may explain the weight-sparing effect observed in these models.

In conclusion, NLG207 in combination with enzalutamide had significant antitumor activity in two different preclinical prostate cancer models harboring clinically relevant mechanisms of enzalutamide resistance. AR-V7 expression, a potentially attractive predictive biomarker, ${ }^{4}$ is ultimately a result of two diverse oncogenic mechanisms driving enzalutamide resistance. AR amplification mediated via AR copy number gain, modeled using $\mathrm{VCaP}$ cells, ${ }^{35,40}$ has been well-characterized in the context of disease progression on enzalutamide in numerous clinical studies. ${ }^{5-7}$ Constitutively active splice variant generation via $\mathrm{AR}$ intragenic gene rearrangement, modeled using 22Rv1 cells, ${ }^{19,21}$ has recently been characterized in clinical CRPC tumors following enzalutamide treatment; intriguingly, AR gene rearrangements were not only shown to generate diverse AR-V species, but to also correlate with AR overexpression in the 
context of AR amplification. ${ }^{20}$ Anti-tumor activity of NLG207 and enzalutamide was demonstrated in both prostate cancer models of AR amplification and AR intragenic rearrangement, suggesting the potential of the treatment combination to effectively target tumors with heterogeneous mechanisms of enzalutamide resistance. Additionally, the treatment combination was effective in the presence of the TMPRSS2-ERG fusion, a tumor characteristic present in nearly half of advanced prostate cancer cases in North America. ${ }^{54}$ Clinical investigation of this treatment combination to confirm anti-tumor activity in patients with mCRPC following disease progression on enzalutamide is currently ongoing (NCT03531827).

Acknowledgment: This study was supported by the Intramural Research Program of the National Institutes of Health, National Cancer Institute, Bethesda, MD, US, (ZIA BC 010547). The content of this publication does not necessarily reflect the views or policies of the Department of Health and Human Services, nor does mention of trade names, commercial products, or organization imply endorsement by the U.S. Government. 


\section{REFERENCES}

1. Teo MY, Rathkopf DE, Kantoff P. Treatment of Advanced Prostate Cancer. Annu Rev Med 2019;70:479-99.

2. Nakazawa M, Paller C, Kyprianou N. Mechanisms of Therapeutic Resistance in Prostate Cancer. Curr Oncol Rep 2017;19:13.

3. Robinson D, Van Allen EM, Wu YM, et al. Integrative clinical genomics of advanced prostate cancer. Cell 2015;161:1215-28.

4. Antonarakis ES, Lu C, Wang H, et al. AR-V7 and resistance to enzalutamide and abiraterone in prostate cancer. N Engl J Med 2014;371:1028-38.

5. Annala M, Vandekerkhove G, Khalaf D, et al. Circulating Tumor DNA Genomics Correlate with Resistance to Abiraterone and Enzalutamide in Prostate Cancer. Cancer Discov 2018;8:444-57.

6. Azad AA, Volik SV, Wyatt AW, et al. Androgen Receptor Gene Aberrations in Circulating Cell-Free DNA: Biomarkers of Therapeutic Resistance in CastrationResistant Prostate Cancer. Clin Cancer Res 2015;21:2315-24.

7. Wyatt AW, Azad AA, Volik SV, et al. Genomic Alterations in Cell-Free DNA and Enzalutamide Resistance in Castration-Resistant Prostate Cancer. JAMA Oncol 2016;2:1598-606.

8. Bharti SK, Kakkad S, Danhier P, et al. Hypoxia Patterns in Primary and Metastatic Prostate Cancer Environments. Neoplasia 2019;21:239-46.

9. Fraga A, Ribeiro R, Principe P, Lopes C, Medeiros R. Hypoxia and Prostate Cancer Aggressiveness: A Tale With Many Endings. Clin Genitourin Cancer 2015;13:295-301.

10. Stewart GD, Ross JA, McLaren DB, Parker CC, Habib FK, Riddick AC. The relevance of a hypoxic tumour microenvironment in prostate cancer. BJU Int 2010;105:8-13.

11. Schito L, Semenza GL. Hypoxia-Inducible Factors: Master Regulators of Cancer Progression. Trends Cancer 2016;2:758-70.

12. Semenza GL. Defining the role of hypoxia-inducible factor 1 in cancer biology and therapeutics. Oncogene 2010;29:625-34.

13. Greer SN, Metcalf JL, Wang Y, Ohh M. The updated biology of hypoxiainducible factor. EMBO J 2012;31:2448-60.

14. Shabsigh A, Ghafar MA, de la Taille A, et al. Biomarker analysis demonstrates a hypoxic environment in the castrated rat ventral prostate gland. J Cell Biochem 2001;81:437-44.

15. Halin S, Hammarsten P, Wikstrom P, Bergh A. Androgen-insensitive prostate cancer cells transiently respond to castration treatment when growing in an androgendependent prostate environment. Prostate 2007;67:370-7.

16. Park C, Kim Y, Shim M, Lee Y. Hypoxia enhances ligand-occupied androgen receptor activity. Biochem Biophys Res Commun 2012;418:319-23.

17. Mitani T, Harada N, Nakano Y, Inui H, Yamaji R. Coordinated action of hypoxia-inducible factor-1alpha and beta-catenin in androgen receptor signaling. J Biol Chem 2012;287:33594-606. 
18. Fernandez EV, Reece KM, Ley AM, et al. Dual targeting of the androgen receptor and hypoxia-inducible factor 1alpha pathways synergistically inhibits castration-resistant prostate cancer cells. Mol Pharmacol 2015;87:1006-12.

19. Li Y, Alsagabi M, Fan D, Bova GS, Tewfik AH, Dehm SM. Intragenic rearrangement and altered RNA splicing of the androgen receptor in a cell-based model of prostate cancer progression. Cancer Res 2011;71:2108-17.

20. Li Y, Yang R, Henzler CM, et al. Diverse AR Gene Rearrangements Mediate Resistance to Androgen Receptor Inhibitors in Metastatic Prostate Cancer. Clin Cancer Res 2020.

21. Li Y, Chan SC, Brand LJ, Hwang TH, Silverstein KA, Dehm SM. Androgen receptor splice variants mediate enzalutamide resistance in castration-resistant prostate cancer cell lines. Cancer Res 2013;73:483-9.

22. Young C, Schluep T, Hwang J, Eliasof S. CRLX101 (formerly IT-101)-A Novel Nanopharmaceutical of Camptothecin in Clinical Development. Curr Bioact Compd 2011;7:8-14.

23. Clark AJ, Wiley DT, Zuckerman JE, et al. CRLX101 nanoparticles localize in human tumors and not in adjacent, nonneoplastic tissue after intravenous dosing. Proc Natl Acad Sci U S A 2016;113:3850-4.

24. Beppu K, Nakamura K, Linehan WM, Rapisarda A, Thiele CJ. Topotecan blocks hypoxia-inducible factor-1alpha and vascular endothelial growth factor expression induced by insulin-like growth factor-I in neuroblastoma cells. Cancer Res 2005;65:4775-81.

25. Rapisarda A, Zalek J, Hollingshead M, et al. Schedule-dependent inhibition of hypoxia-inducible factor-1alpha protein accumulation, angiogenesis, and tumor growth by topotecan in U251-HRE glioblastoma xenografts. Cancer Res 2004;64:6845-8. 26. Lin CJ, Lin YL, Luh F, Yen Y, Chen RM. Preclinical effects of CRLX101, an investigational camptothecin-containing nanoparticle drug conjugate, on treating glioblastoma multiforme via apoptosis and antiangiogenesis. Oncotarget 2016;7:4240821.

27. Conley SJ, Baker TL, Burnett JP, et al. CRLX101, an investigational camptothecin-containing nanoparticle-drug conjugate, targets cancer stem cells and impedes resistance to antiangiogenic therapy in mouse models of breast cancer. Breast Cancer Res Treat 2015;150:559-67.

28. Pham E, Birrer MJ, Eliasof S, et al. Translational impact of nanoparticle-drug conjugate CRLX101 with or without bevacizumab in advanced ovarian cancer. Clin Cancer Res 2015;21:808-18.

29. Pham E, Yin M, Peters CG, et al. Preclinical Efficacy of Bevacizumab with CRLX101, an Investigational Nanoparticle-Drug Conjugate, in Treatment of Metastatic Triple-Negative Breast Cancer. Cancer Res 2016;76:4493-503.

30. Markowski MC, Silberstein JL, Eshleman JR, Eisenberger MA, Luo J, Antonarakis ES. Clinical Utility of CLIA-Grade AR-V7 Testing in Patients With Metastatic Castration-Resistant Prostate Cancer. JCO Precis Oncol 2017;2017. 31. Font-Tello A, Juanpere N, de Muga S, et al. Association of ERG and TMPRSS2ERG with grade, stage, and prognosis of prostate cancer is dependent on their expression levels. Prostate 2015;75:1216-26. 
32. Liu C, Lou W, Zhu Y, et al. Niclosamide inhibits androgen receptor variants expression and overcomes enzalutamide resistance in castration-resistant prostate cancer. Clin Cancer Res 2014;20:3198-210.

33. Liu C, Lou W, Zhu Y, et al. Intracrine Androgens and AKR1C3 Activation Confer Resistance to Enzalutamide in Prostate Cancer. Cancer Res 2015;75:1413-22. 34. Schluep T, Hwang J, Cheng J, et al. Preclinical efficacy of the camptothecinpolymer conjugate IT-101 in multiple cancer models. Clin Cancer Res 2006;12:1606-14. 35. Liu LL, Xie N, Sun S, Plymate S, Mostaghel E, Dong X. Mechanisms of the androgen receptor splicing in prostate cancer cells. Oncogene 2014;33:3140-50.

36. Moilanen AM, Riikonen R, Oksala R, et al. Discovery of ODM-201, a newgeneration androgen receptor inhibitor targeting resistance mechanisms to androgen signaling-directed prostate cancer therapies. Sci Rep 2015;5:12007.

37. Luo J, Li Y, Zheng W, et al. Characterization of a Prostate- and Prostate CancerSpecific Circular RNA Encoded by the Androgen Receptor Gene. Mol Ther Nucleic Acids 2019;18:916-26.

38. Kregel S, Chen JL, Tom W, et al. Acquired resistance to the second-generation androgen receptor antagonist enzalutamide in castration-resistant prostate cancer. Oncotarget 2016;7:26259-74.

39. Cai C, He HH, Chen S, et al. Androgen receptor gene expression in prostate cancer is directly suppressed by the androgen receptor through recruitment of lysinespecific demethylase 1. Cancer Cell 2011;20:457-71.

40. Cai C, Wang H, Xu Y, Chen S, Balk SP. Reactivation of androgen receptorregulated TMPRSS2:ERG gene expression in castration-resistant prostate cancer. Cancer Res 2009;69:6027-32.

41. Asangani IA, Wilder-Romans K, Dommeti VL, et al. BET Bromodomain Inhibitors Enhance Efficacy and Disrupt Resistance to AR Antagonists in the Treatment of Prostate Cancer. Mol Cancer Res 2016;14:324-31.

42. Fruehauf JP, Farrokhian N, Sarkissian S, Kim JH. Blockade of ARV7:

HIF1alpha heterodimers after topotecan reverses enzalutamide resistance in 22Rv1 cells. J Clin Oncol 2016;34(15_suppl):e16594.

43. Chiang KC, Tsui $\mathrm{KH}$, Chung LC, et al. Topoisomerase inhibitors modulate gene expression of B-cell translocation gene 2 and prostate specific antigen in prostate carcinoma cells. PLoS One 2014;9:e89117.

44. Liu S, Yuan Y, Okumura Y, Shinkai N, Yamauchi H. Camptothecin disrupts androgen receptor signaling and suppresses prostate cancer cell growth. Biochem Biophys Res Commun 2010;394:297-302.

45. Rapisarda A, Uranchimeg B, Sordet O, Pommier Y, Shoemaker RH, Melillo G. Topoisomerase I-mediated inhibition of hypoxia-inducible factor 1: mechanism and therapeutic implications. Cancer Res 2004;64:1475-82.

46. Minelli R, Cavalli R, Ellis L, et al. Nanosponge-encapsulated camptothecin exerts anti-tumor activity in human prostate cancer cells. Eur J Pharm Sci 2012;47:68694.

47. Gaur S, Wang Y, Kretzner L, et al. Pharmacodynamic and pharmacogenomic study of the nanoparticle conjugate of camptothecin CRLX101 for the treatment of cancer. Nanomedicine 2014;10:1477-86. 
48. St John J, Powell K, Conley-Lacomb MK, Chinni SR. TMPRSS2-ERG Fusion Gene Expression in Prostate Tumor Cells and Its Clinical and Biological Significance in Prostate Cancer Progression. J Cancer Sci Ther 2012;4:94-101.

49. Mertz KD, Setlur SR, Dhanasekaran SM, et al. Molecular characterization of TMPRSS2-ERG gene fusion in the NCI-H660 prostate cancer cell line: a new perspective for an old model. Neoplasia 2007;9:200-6.

50. Roche E, Montaudon D, Kayali S, Houede N, Pourquier P. Role of the ERG transcription factor in the resistance of prostate cancer cells to the topoisomerase I inhibitor camptothecin. Cancer Res 2014;74(19 Suppl):Abstract nr 3813.

51. Semaan L, Mander N, Cher ML, Chinni SR. TMPRSS2-ERG fusions confer efficacy of enzalutamide in an in vivo bone tumor growth model. BMC Cancer 2019;19:972.

52. Grande E, Fernandez Perez MP, Wetterskog D, et al. A phase II multicenter biomarker trial to study the predictive value of TMPRSS2-ERG before enzalutamide treatment in chemo-naïve metastatic castration-resistant prostate cancer. J Clin Oncol 2019;37, no. 15_suppl:5040.

53. Han W, Gao S, Barrett D, et al. Reactivation of androgen receptor-regulated lipid biosynthesis drives the progression of castration-resistant prostate cancer. Oncogene 2018;37:710-21.

54. Zhou CK, Young D, Yeboah ED, et al. TMPRSS2:ERG Gene Fusions in Prostate Cancer of West African Men and a Meta-Analysis of Racial Differences. Am J Epidemiol 2017;186:1352-61. 


\section{FIGURES}

Figure 1. Effect of NLG207, camptothecin, and enzalutamide on cell proliferation of $22 \mathrm{Rv} 1$ cells.

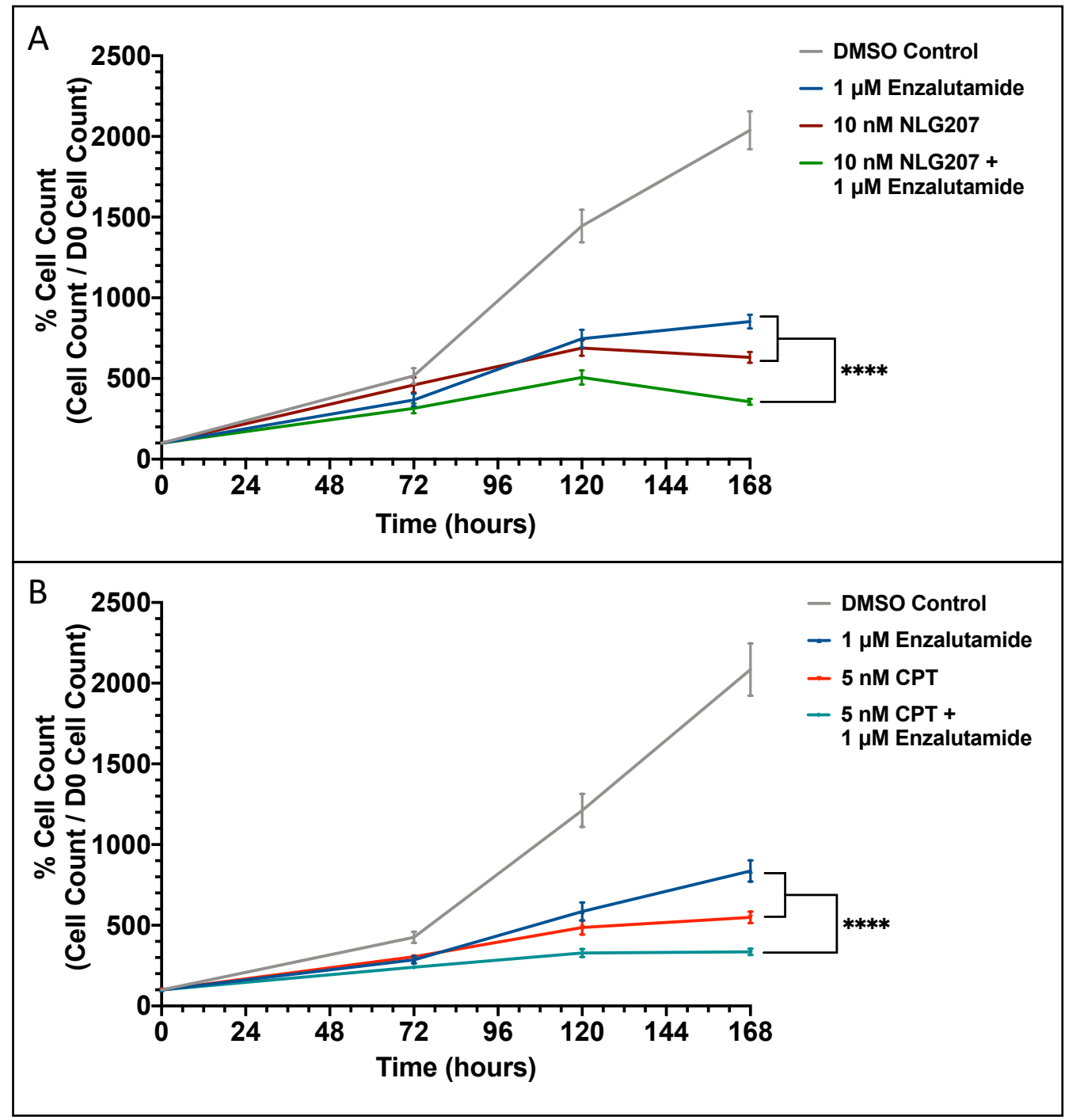

Growth of 22Rv1 cells in media containing $0.1 \mathrm{nM} \mathrm{R} 1881$ with or without $1 \mu \mathrm{M}$ enzalutamide was evaluated over 168 hours following treatment with $10 \mathrm{nM}$ NLG207 (A) or $5 \mathrm{nM}$ camptothecin (B). Cell viability was determined using the Cell Counting Kit-8 cell viability assay at the indicated time points. The result is representative of three independent experiments. Statistical significance between groups by day 7 is denoted: $* * * *-\mathrm{p}<0.0001$. 
Figure 2. Efficacy of NLG207 and enzalutamide in subcutaneous 22Rv1 xenografts after 3 weeks of treatment.

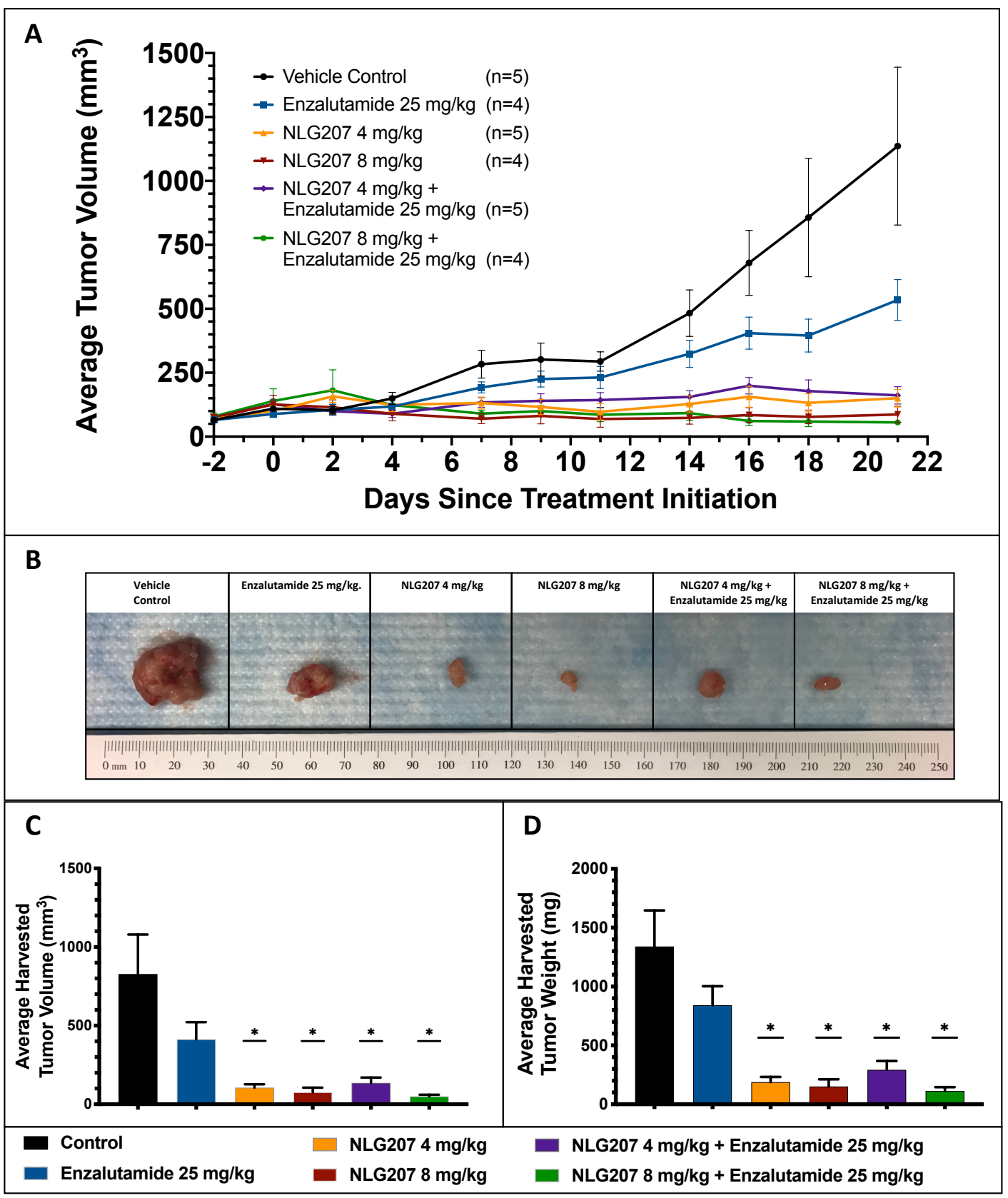




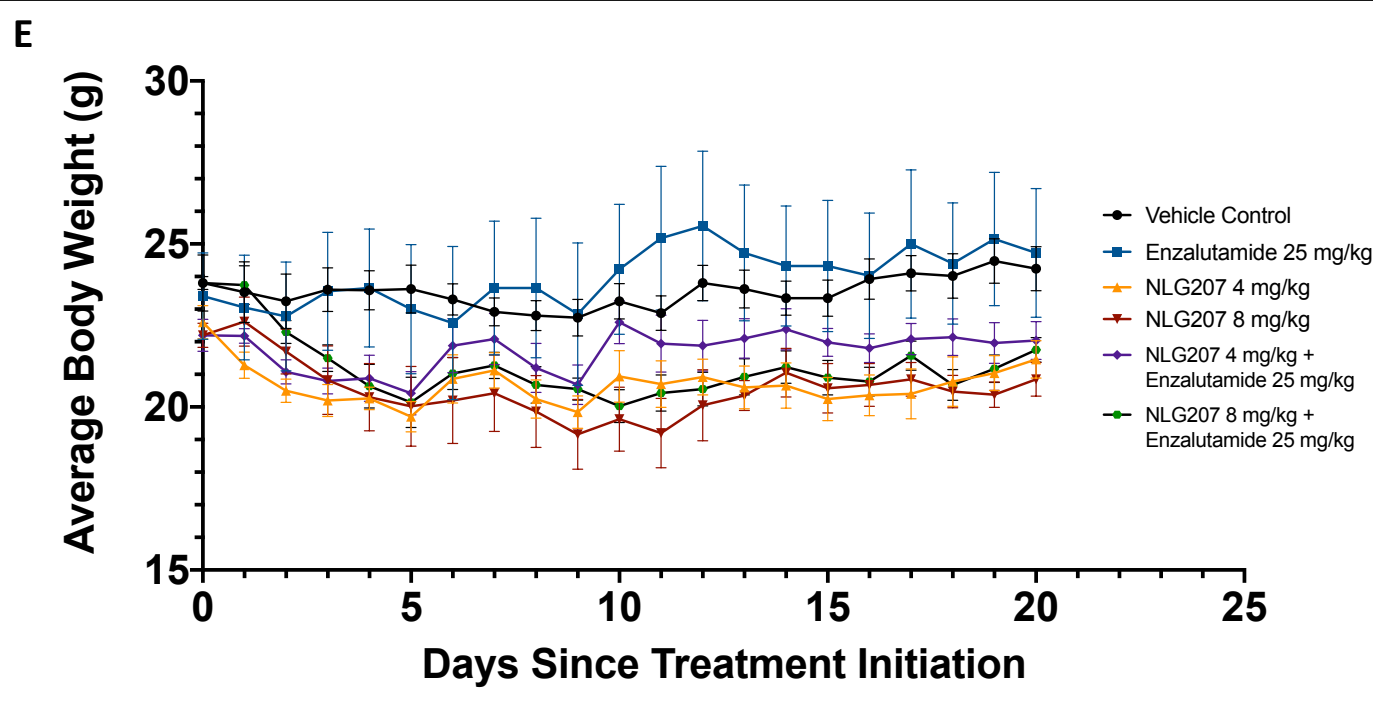

Mice with subcutaneous 22Rv1 xenografts were treated for 3 weeks with the specified doses of vehicle controls, enzalutamide and/or NLG207, and euthanized at the completion of treatment. Average tumor volume measurements collected are plotted during the course of the study (A). Tumors, harvested following 3 weeks of treatment, were compared, as shown via photograph with one tumor per group (B), average tumor volume (C) and average tumor weight (D) at time of harvest; statistical comparisons to vehicle control above each treatment group are denoted: * $-\mathrm{p}<0.05$. Daily average body weight measurements collected are summarized over the course of the study $(\mathbf{E})$. 
Figure 3. Efficacy of NLG207 and enzalutamide in subcutaneous 22Rv1 xenografts, as measured by tumor re-growth rate.

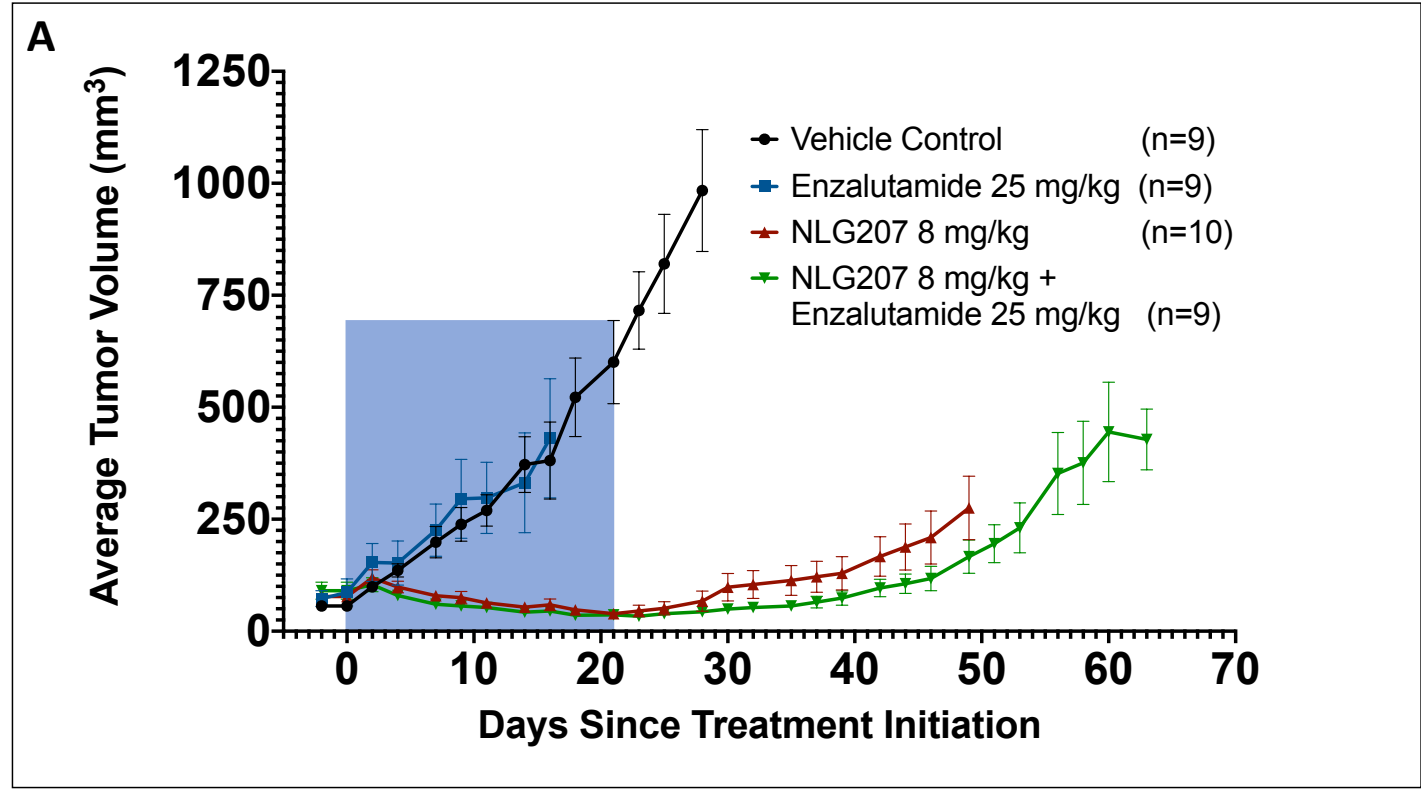

B

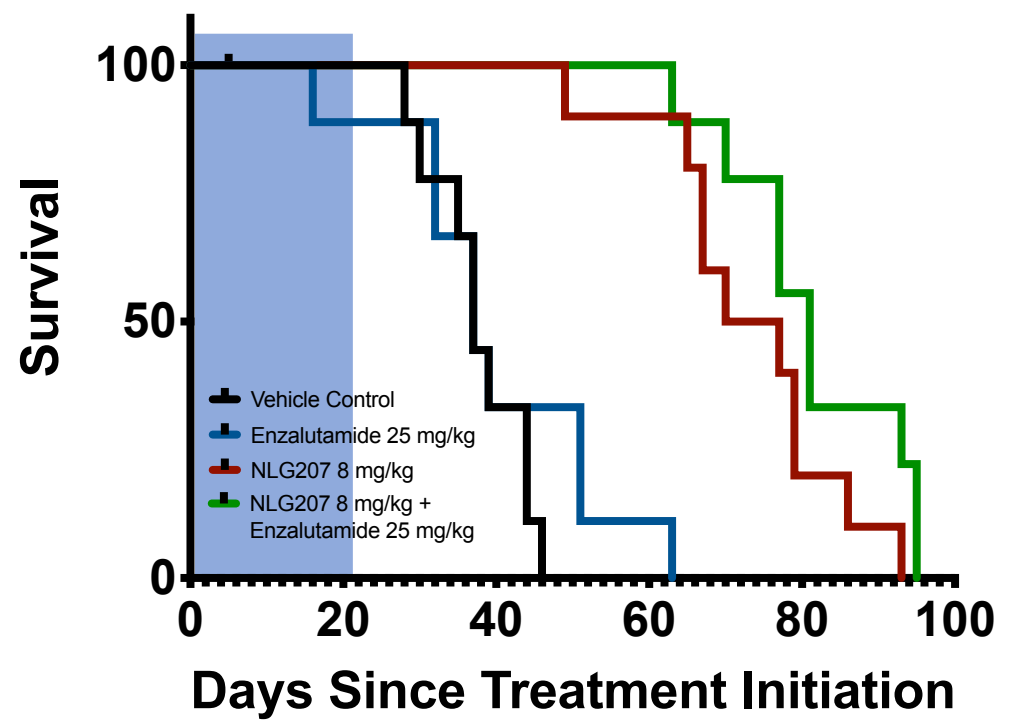




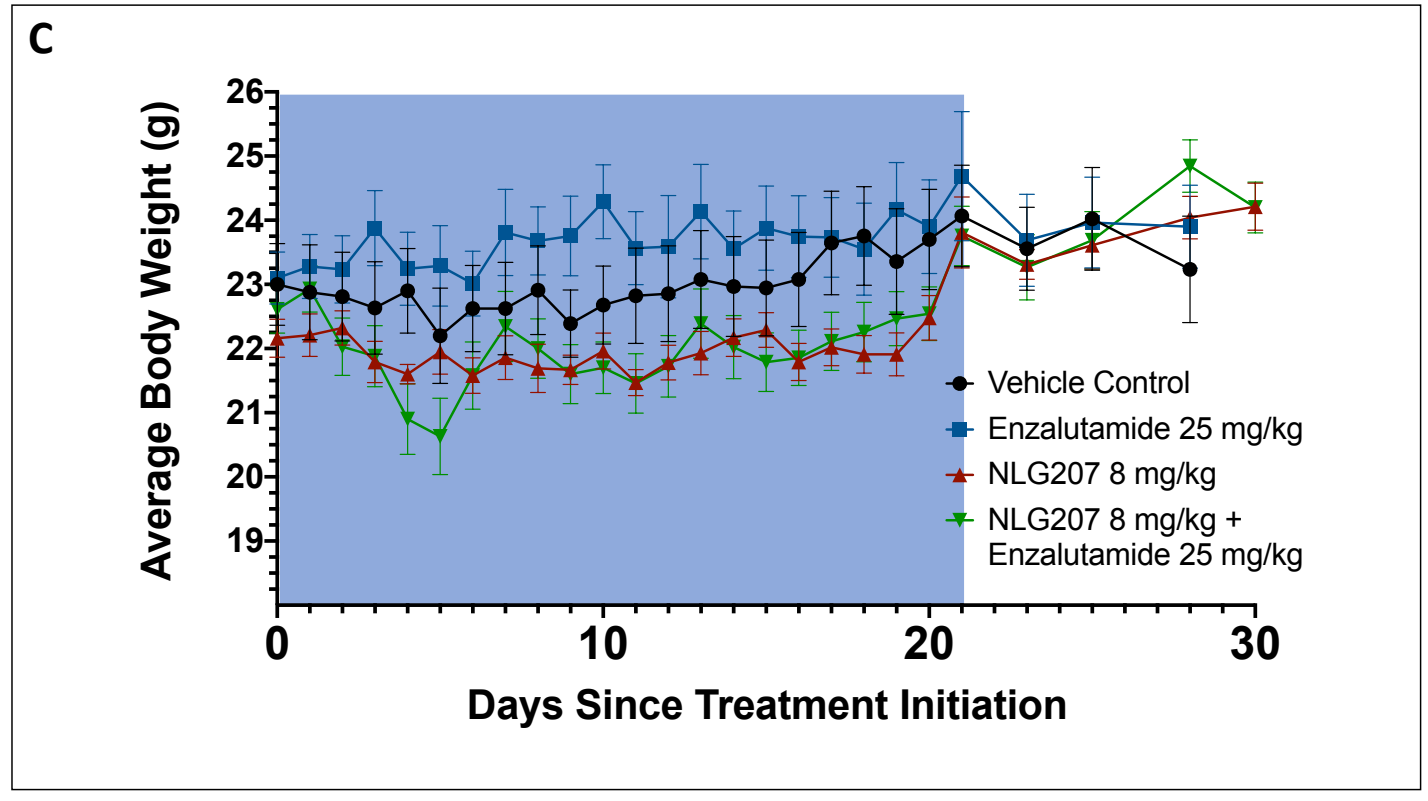

Mice with subcutaneous 22Rv1 xenografts were treated for 3 weeks with specified doses of vehicle control, enzalutamide and/or NLG207, then monitored for tumor re-growth. Average tumor volume measurements collected are plotted over the course of the study, with a comparison of NLG207 treated vs. non-NLG207 treated groups (A). KaplanMeier survival analysis summarizes time to removal from study, with censoring of animals removed for $>20 \%$ body weight reduction. (B). Average body weight measurements per treatment group collected through the first 30 days of the study are summarized $(\mathbf{C})$. The blue shading indicates the time frame of on-study drug treatment. 
Figure 4. In vitro anti-tumor activity of NLG207 and enzalutamide in VCaP cells.

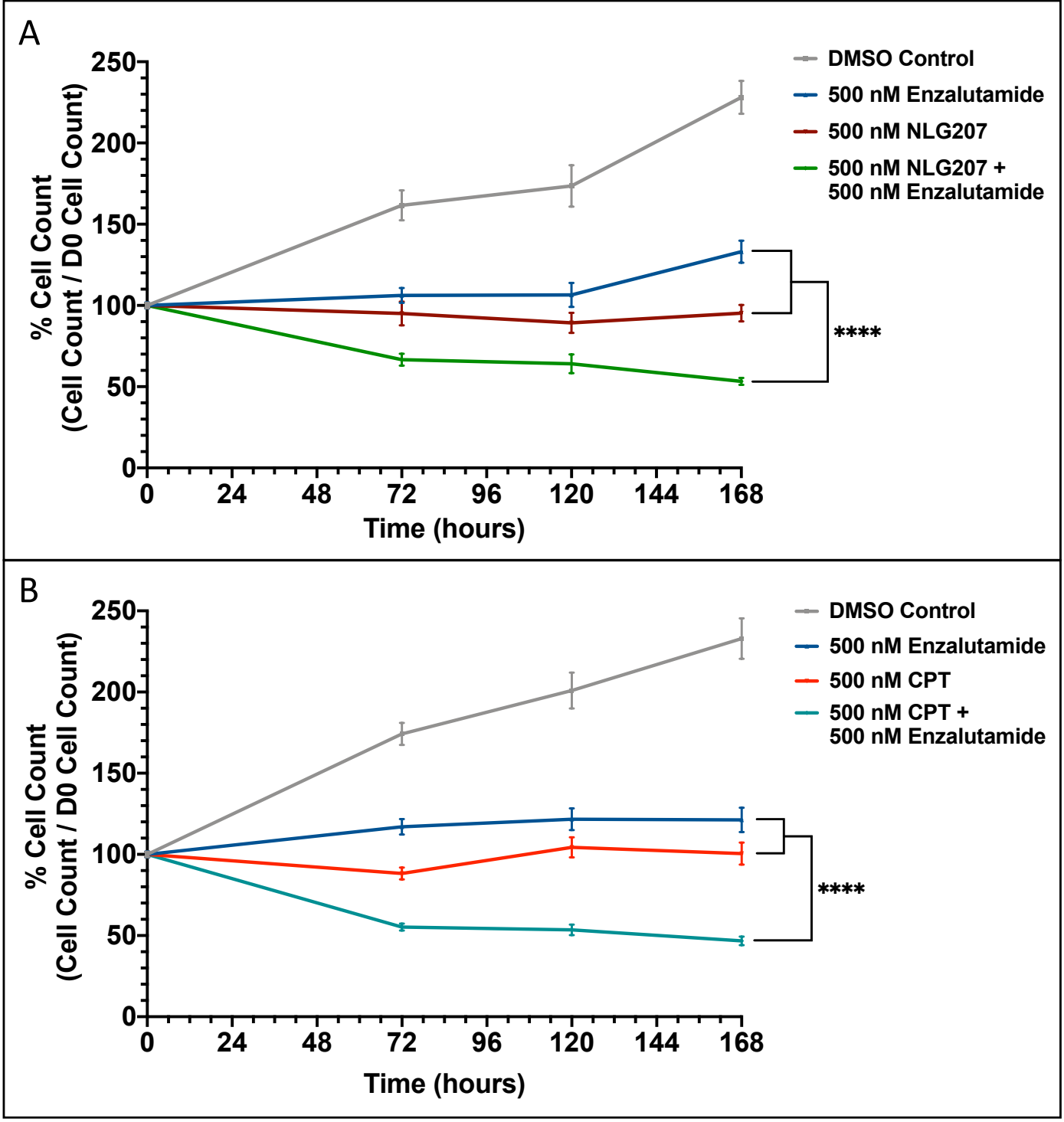




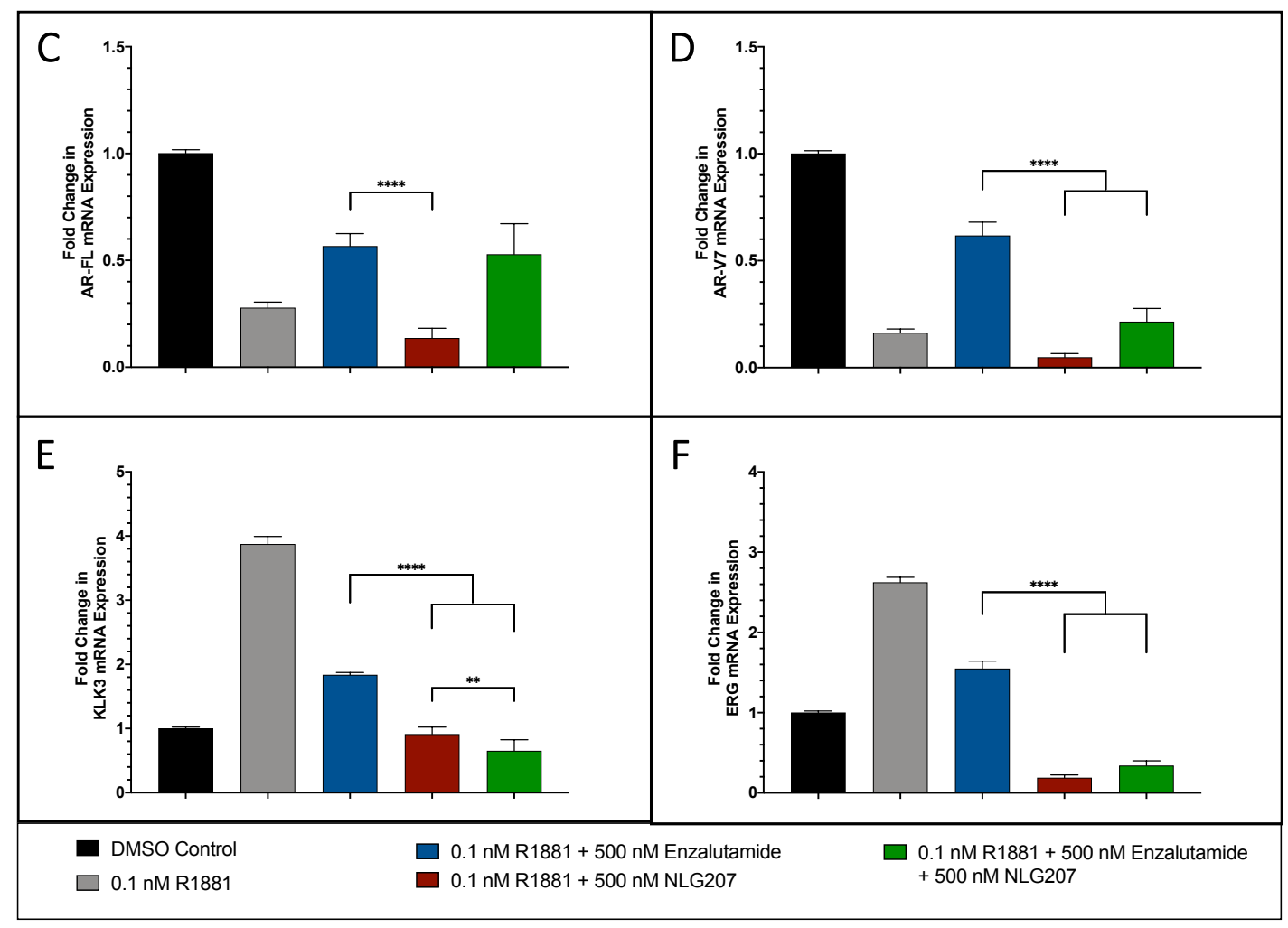

Growth of VCaP cells in media containing $0.1 \mathrm{nM} \mathrm{R} 1881$ with or without $500 \mathrm{nM}$ enzalutamide was evaluated over 168 hours following treatment with $500 \mathrm{nM}$ NLG207 (A) or $500 \mathrm{nM}$ camptothecin (B). Cell viability was determined using the Cell Counting Kit-8 cell viability assay at the indicated time points. The fold change in mRNA expression of AR-FL (C), AR-V7 (D), KLK3 (E), ERG (F) following 24 hours of treatment with combinations of R1881, enzalutamide and NLG207 compared to cells in $10 \%$ CDS-FBS supplemented media. Results are representative of three independent experiments. Significance of indicated comparisons are denoted: $* *-p<0.01, * * * *-p$ $<0.0001$. 
Figure 5. Efficacy of NLG207 and enzalutamide in castrated subcutaneous VCaP xenografted mice.
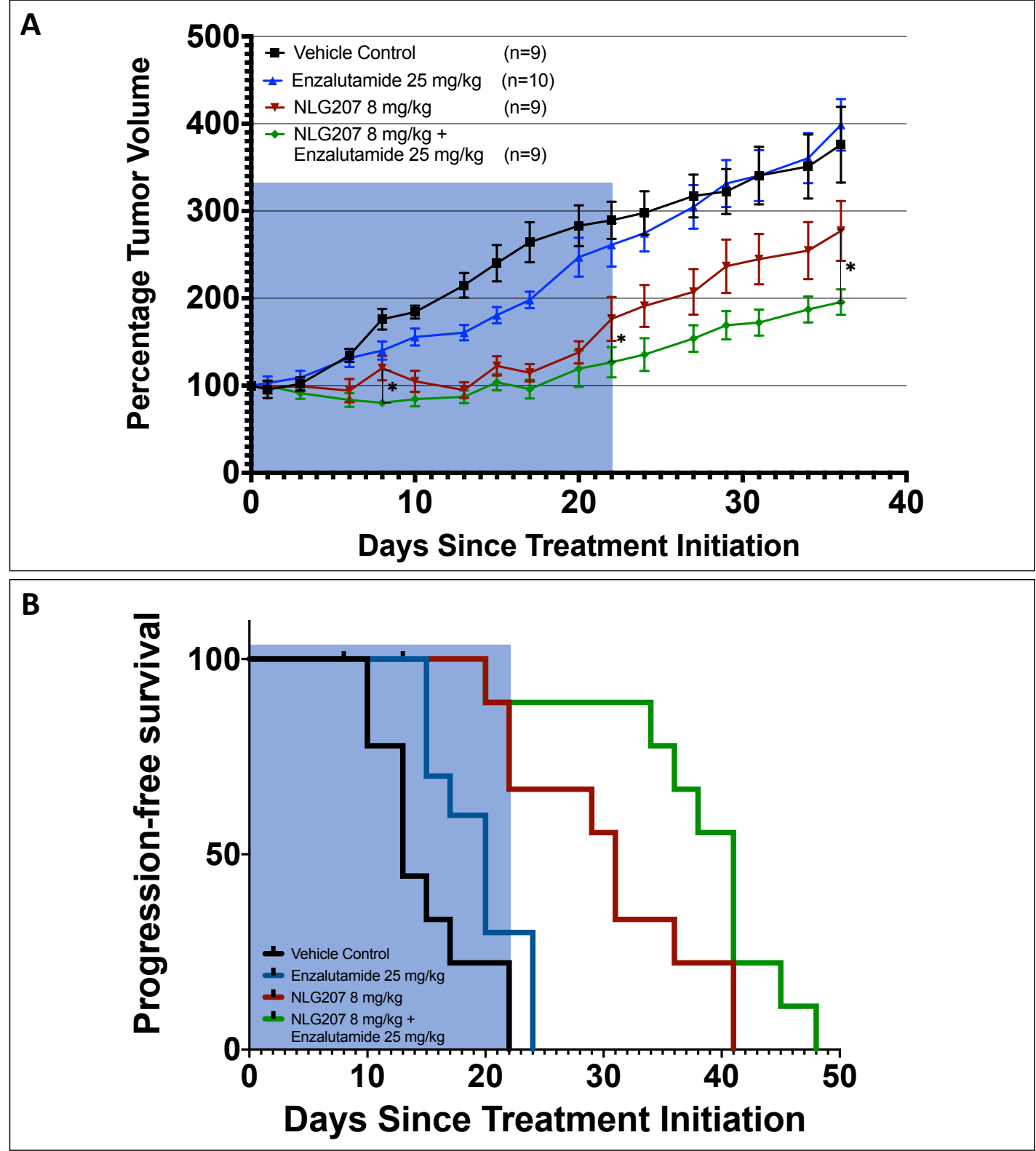


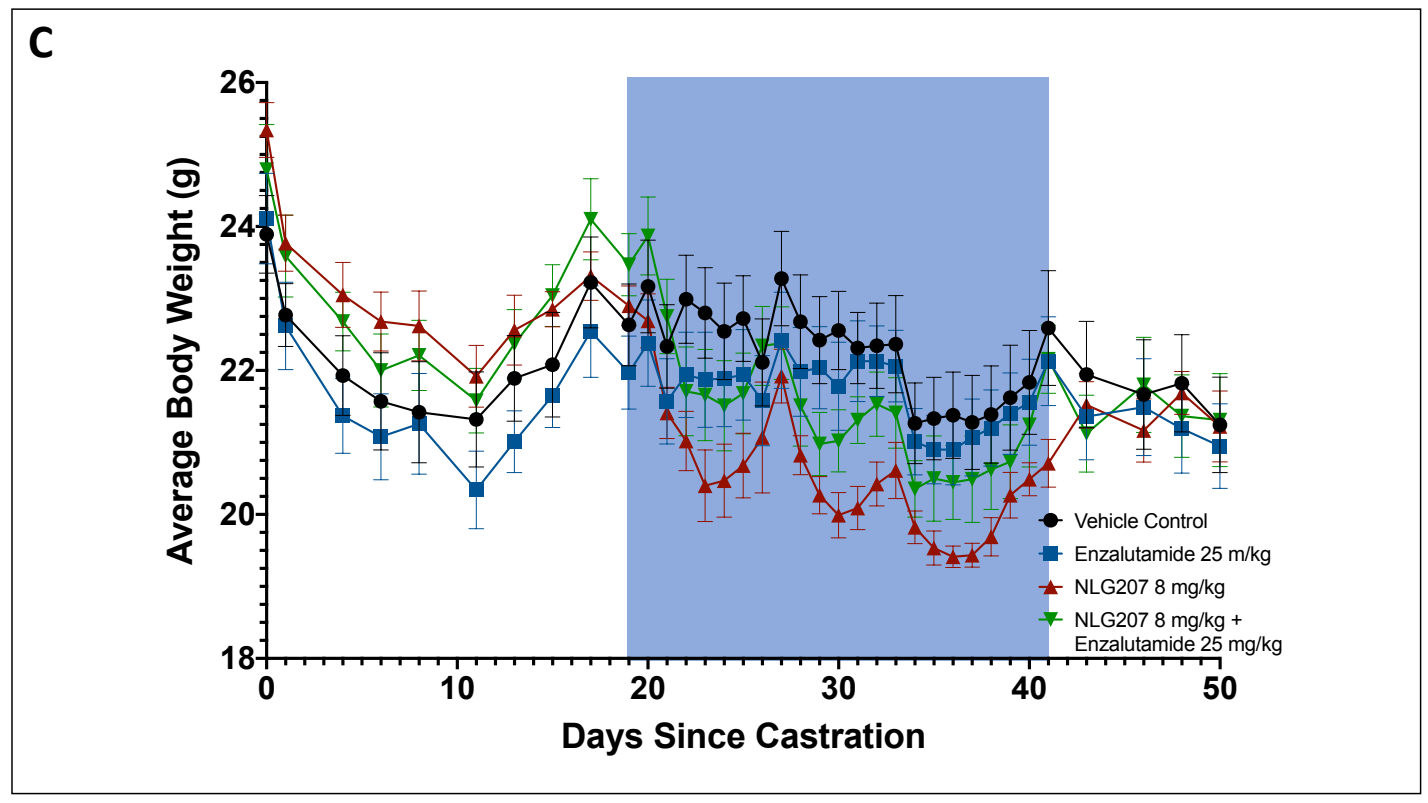

Castrated mice with subcutaneous VCaP xenografts were treated for 3 weeks with specified doses of vehicle control, enzalutamide, and/or NLG207, then monitored for tumor re-growth. Average percent change in tumor volume measurements (baseline at the start of treatment) through day 36 post-treatment initiation are summarized, with statistical significance of comparisons at specified time points denoted: * $-p \leq 0.05$ (A). Kaplan-Meier survival analysis progression-free survival (progression indicated when a tumor doubled in size) for each treatment group (B). Mice were censored if treatment resulted in $>20 \%$ reduction in body weight. Average body weight measurements per treatment group collected following castration through 10 days post-drug treatment $(\mathbf{C})$. The blue shading indicates the time frame for on-study drug treatment. 


\section{SUPPLEMENTARY FIGURES}

Figure S1. qPCR primer validation for AR-FL and AR-V7.

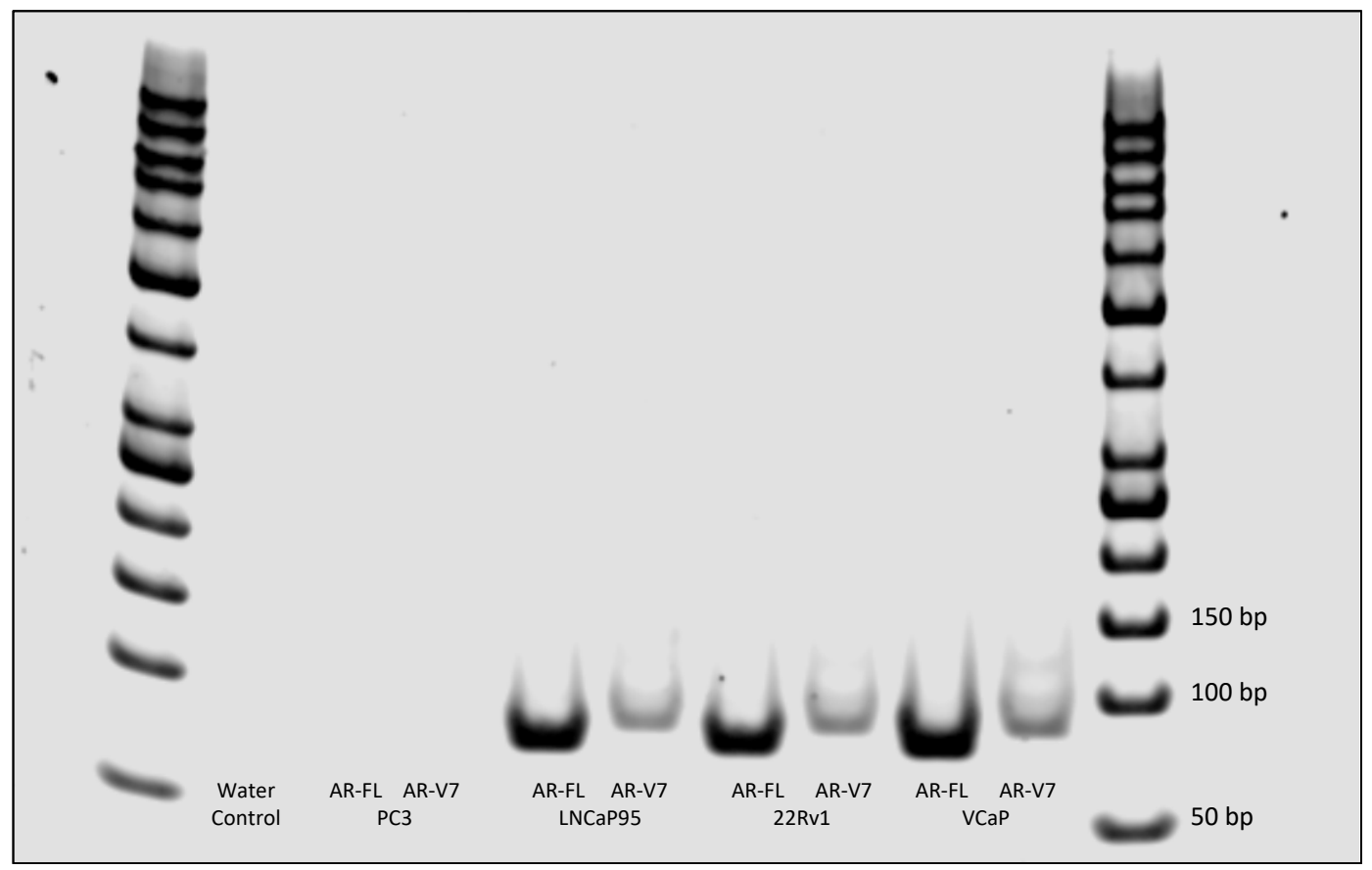

Gel electrophoretic separation (4-20\% TBE) of DNA fragments generated from the ARFL and AR-V7 qPCR primers (adapted from Markowski et al.) ${ }^{30}$ in four prostate cancer cell lines following $30 \mathrm{qPCR}$ cycles. 
Figure S2. Effect of camptothecin and enzalutamide on mRNA expression of relevant AR pathway associated genes in $\mathrm{VCaP}$ cells.

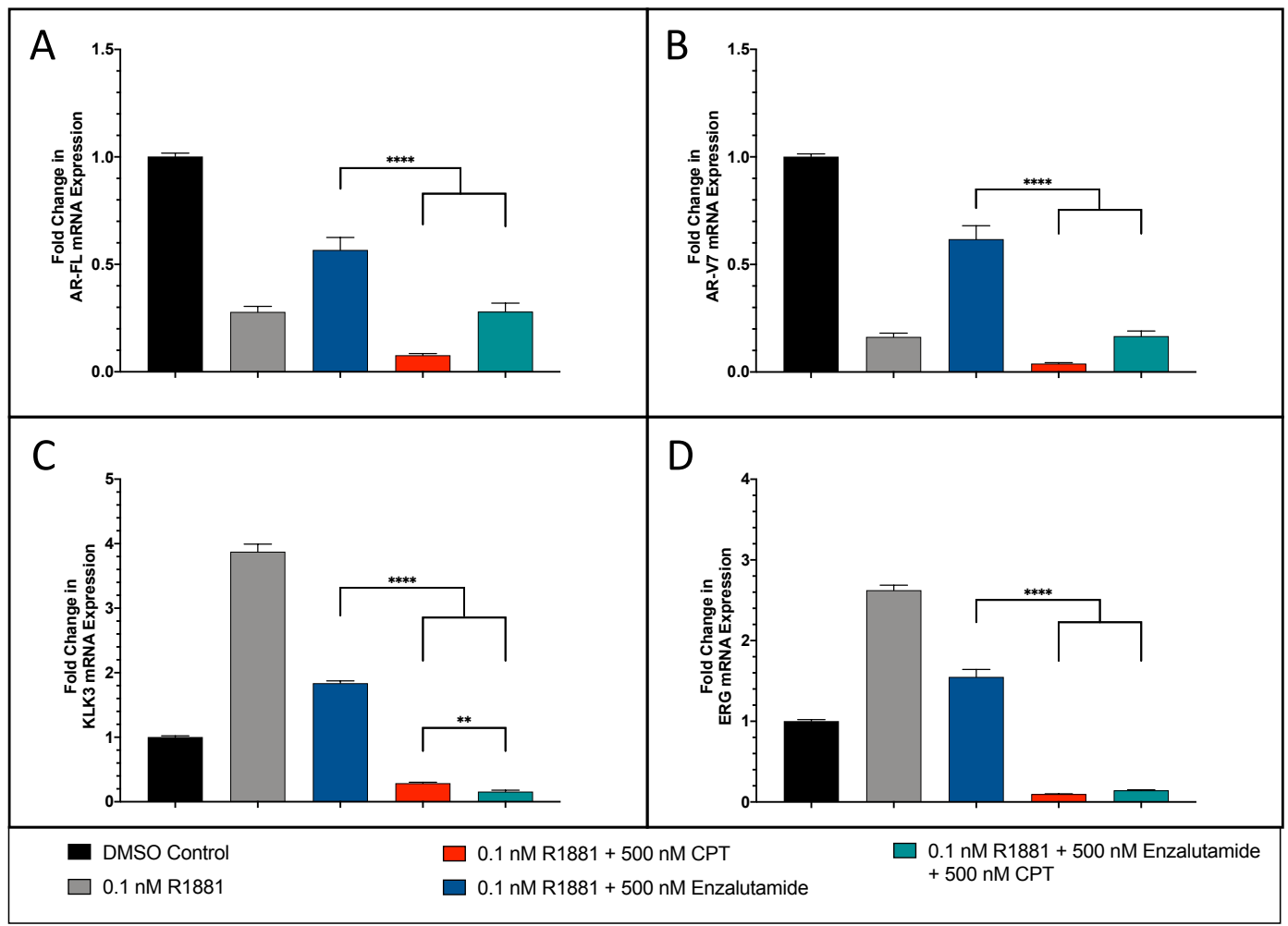

The fold change in mRNA expression of AR-FL (A), AR-V7 (B), KLK3 (C), and ERG (D) following 24 hours of treatment with combinations of R1881, enzalutamide and camptothecin compared to cells in 10\% CDS-FBS supplemented media. The result is representative of three independent experiments. Significance of indicated comparisons are denoted via brackets: $* *-\mathrm{p}<0.01, * * * *-\mathrm{p}<0.0001$. 
Figure S3. Effect of NLG207, camptothecin, and enzalutamide on mRNA expression of relevant HIF-1 $\alpha$ pathway associated genes in VCaP cells.

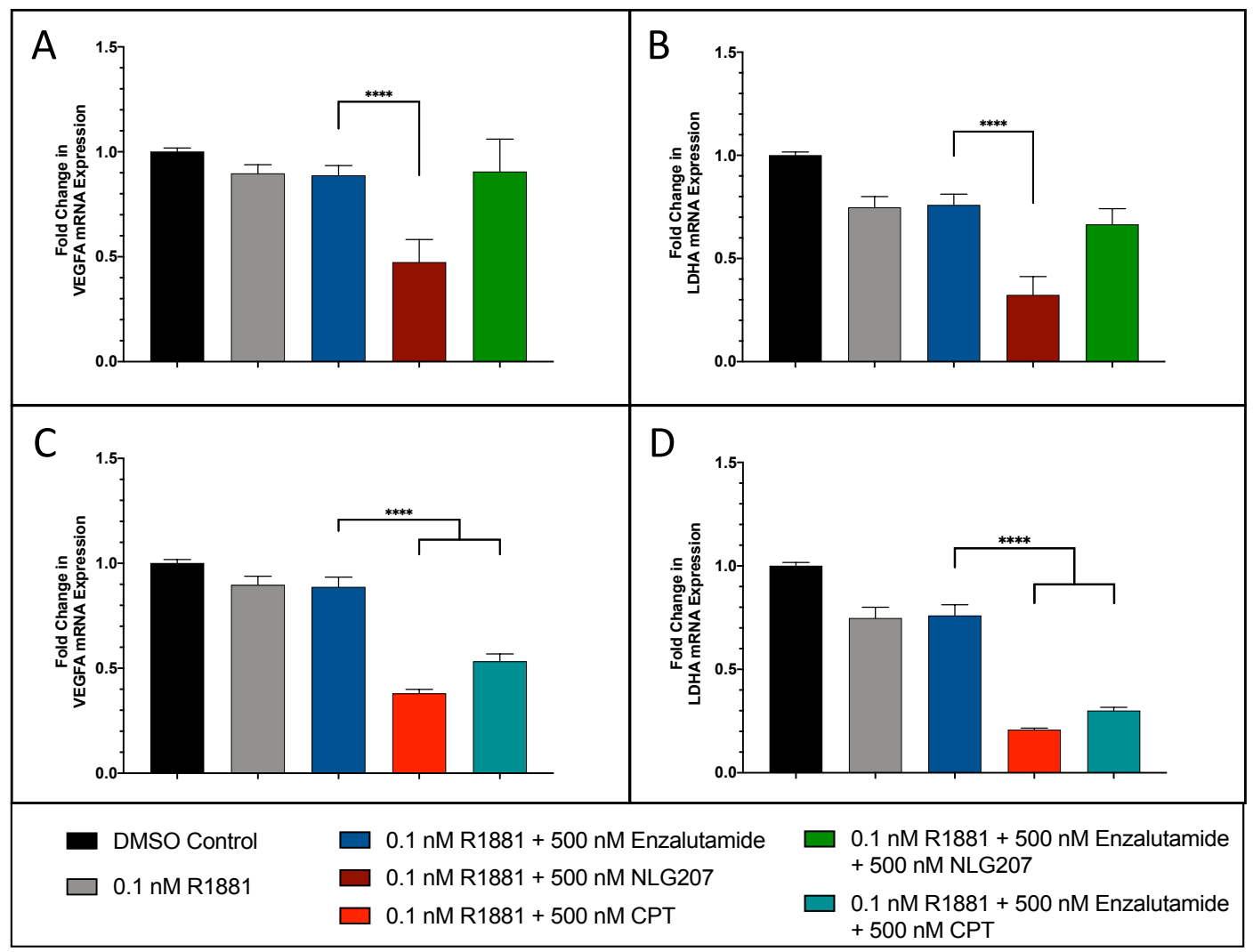

The fold change in mRNA expression of VEGFA (A \& C) and LDHA (B \& D) following 24 hours of treatment with combinations of R1881, enzalutamide, NLG207 and camptothecin compared to cells in 10\% CDS-FBS supplemented media. The result is representative of three independent experiments. Significance of indicated comparisons are denoted via brackets: $* * * *-p<0.0001$. 


\section{Chapter 5}

\section{Measurement of NLG207 (formerly CRLX101) Nanoparticle-bound and Released Camptothecin in Human Plasma}

Keith T. Schmidt ${ }^{1}$, Cody J. Peer ${ }^{1}$, Alwin D. R. Huitema ${ }^{2,3}$ Monique D. Williams ${ }^{4}$, Susan Wroblewski ${ }^{4}$, Jan H. M. Schellens ${ }^{5}$, Ravi A. Madan ${ }^{4}$, and William D. Figg ${ }^{1,4}$

${ }^{1}$ Clinical Pharmacology Program, Center for Cancer Research, National Cancer Institute, National Institutes of Health, Bethesda, MD

${ }^{2}$ Dept. Pharmacy \& Pharmacology, Netherlands Cancer Institute, Amsterdam, The Netherlands

${ }^{3}$ Department of Clinical Pharmacy, University Medical Center Utrecht, Utrecht University, Utrecht, The Netherlands

${ }^{4}$ Genitourinary Malignancies Branch, Center for Cancer Research, National Cancer Institute, National Institutes of Health, Bethesda, MD, USA

${ }^{5}$ Science Faculty, Utrecht University, Utrecht, The Netherlands

J Pharm Biomed Anal. 2020;181:113073 


\section{Abstract}

Camptothecin (CPT), a potent inhibitor of topoisomerase I and HIF-1 $\alpha$, failed to demonstrate utility as an anti-cancer agent in early clinical trial investigations, primarily due to limited clinical activity and significant toxicity attributable to unfavorable physicochemical properties (e.g. low plasma solubility, pH-labile lactone ring). NLG207 (formerly CRLX101), a nanoparticle-drug conjugate (NDC) of CPT designed to optimize plasma pharmacokinetics and facilitate drug delivery to tumors, is included as part of combination treatment in two Phase II clinical trials ongoing at the National Cancer Institute (NCT02769962 and NCT03531827). To better understand the potential for drug-drug interactions and to correlate drug exposure to clinical outcomes and pharmacodynamic biomarkers, a robust analytical method was developed to measure CPT in human plasma. Two sample processing methods were developed to quantify both NDC-bound CPT and free CPT, primarily via alteration of $\mathrm{pH}$ conditions. A solidphase extraction recovered $>79 \%$ of CPT prior to quantitative analysis by UPLCMS/MS. Dynamic calibration ranges of 10 to $10,000 \mathrm{ng} / \mathrm{mL}$ and 1 to $1,000 \mathrm{ng} / \mathrm{mL}$ for total and free CPT, respectively were expected to capture clinical ranges. NLG207 NDCs demonstrated significant rates of CPT release in human plasma at room temperature after 2 hours but were shown to be stable at $4^{\circ} \mathrm{C}$ for 24 hours and through 4 freeze/thaw cycles. This assay was used to quantitate CPT plasma concentrations in clinical samples to confirm clinical utility following NLG207 treatment in subjects with advanced prostate cancer. 


\section{Highlights}

- NLG207 is a nanoparticle-drug conjugate of camptothecin (topoisomerase I inhibitor).

- Analytical methods were developed to measure nanoparticle-bound \& free camptothecin.

- The pharmacokinetic assay to quantitate plasma concentrations was accurate \& precise.

- NLG207 samples were stable up to $24 \mathrm{~h}$ at $4{ }^{\circ} \mathrm{C}$ and following 4 freeze-thaw cycles.

- Clinical utility was shown via quantitation of samples from NLG207-treated patients. 


\section{Introduction}

NLG207 (formerly CRLX101) is a nanoparticle-drug conjugate (NDC) of the potent topoisomerase I inhibitor, camptothecin (CPT), developed to overcome issues associated with poor plasma solubility ${ }^{1-3}$ and enhance drug delivery to tumor tissue

(Figure 1).${ }^{4-6}$ Following deposition into tumors, the rate of CPT release is controlled via pH-dependent hydrolysis of the carboxyl ester linkers while retaining CPT in the active lactone form. ${ }^{6,7}$ The anti-tumor activity of NLG207, both as monotherapy and in combination with other agents, has been characterized in numerous preclinical models, ${ }^{8-}$ 12 with enhanced efficacy demonstrated in comparison to small molecule CPT and irinotecan. ${ }^{13}$

To date, the treatment of over 304 patients with at least one dose of NLG207 on multiple clinical trials has been reported in the literature. ${ }^{7,9,14-17}$ Utility of NLG207 has spanned across multiple tumor histologies, including advanced non-small cell lung cancer, advanced ovarian cancer, and metastatic renal cell carcinoma. The most commonly utilized Phase II dose of NLG207 is $15 \mathrm{mg} / \mathrm{m}^{2}$ given via intravenous infusion every two weeks, given either as monotherapy or as combination therapy (e.g. bevacizumab), though lower $\left(12 \mathrm{mg} / \mathrm{m}^{2}\right)$ or more frequent (weekly) dosing strategies have been explored. Characterization of clinical plasma pharmacokinetics via noncompartmental analysis has been conducted using serial samples collected over two weeks from the initial Phase I/II clinical trial (samples quantitated by an unpublished LC/MS method by the company). Pharmacokinetic parameters have been reported for both CPT bound to NLG207 (conjugated) and free CPT (unconjugated), with a relative 11-fold increase in exposure (determined via $\mathrm{AUC}_{\mathrm{LAST}}$ ) of conjugated over 
unconjugated CPT. Mean clearance and volume of distribution values were found to be dose-independent, with volume of distribution values of conjugated CPT suggesting retention predominantly in the vasculature and highly perfused tissues $(2.33$ to $4.63 \mathrm{~L}){ }^{7}$

Herein we describe a novel, robust, selective, accurate and precise bioanalytical method for the quantification of CPT, both conjugated and unconjugated to NLG207 NDCs. Previous analytical methods, though accurate and precise, proved to be inefficient. This assay was developed and validated according to FDA guidelines, while also incorporating a solid phase extraction step, including an internal standard (SN-38), and eliminating the use of strong acid in sample preparation to reduce LC column degradation. To further improve assay validation consistency and reliability from run to run, the quantitation ranges were validated between 10 to $10,000 \mathrm{ng} / \mathrm{mL}$ and 1 to 1,000 $\mathrm{ng} / \mathrm{mL}$ for total CPT and free CPT quantitation, respectively, which are reflective of clinically relevant drug concentrations. ${ }^{7}$ This assay was demonstrated to be applicable for clinical pharmacokinetic purposes by using the small patient cohorts of NCT02769962 and NCT03531827 (subsequent pharmacokinetic data to be published separately), and used to assess the stability of NDCs in clinically relevant scenarios.

\section{Materials and methods}

\subsection{Materials}

S-(+)-Campothecin was provided by Millipore Sigma (Rockville, MD), SN-38 was provided by Selleck Chemicals (Houston, TX), and NLG207 was provided by NewLink Genetics (Ames, IA). The chemical structures of camptothecin and SN-38, analyte and internal standard respectively, are shown in Figure S1. Formic acid and 
sodium hydroxide were provided by Millipore Sigma (Rockville, MD). Optima-grade methanol was obtained from Fisher Scientific (Fairlawn, NJ), and a Hydro-Reverse Osmosis system (Durham, NC) linked to a Milli-Q UV Plus purifying system (Billerica, MA) was used to generate de-ionized water. Drug-free human heparinized plasma and whole blood was obtained from the NIH Clinical Center Blood Bank (Bethesda, MD).

\subsection{Total CPT quantitation}

The purpose of this preparative method was to force-release all CPT from NLG207 NDCs using high $\mathrm{pH}$ conditions ${ }^{18}$ and quantitate the total CPT present in a patient sample.

\subsubsection{Preparation of stock solutions}

Clinical-grade vials of NLG207 (35 mg CPT equivalents) were re-constituted in Dulbecco's phosphate buffered saline (DPBS) to a concentration of $1 \mathrm{mg} / \mathrm{mL}$, aliquoted in glass vials, and stored in $-20^{\circ} \mathrm{C}$. To ensure NDC stability following multiple freezethaws, a previously unused (i.e. one freeze-thaw) $1 \mathrm{mg} / \mathrm{mL}$ vial was used to generate serial dilutions (working stocks) in DPBS for each individual run. The working stocks were then used for preparation of calibration and quality control (QC) samples. The SN38 Master Stock at a concentration of $1 \mathrm{mg} / \mathrm{mL}$ was prepared individually via dissolution in DMSO, vortex mixing, brief sonication, and storage at $-80^{\circ} \mathrm{C}$.

Calibration standards were freshly prepared in drug-free human heparinized plasma at concentrations of $10,50,100,500,1000,5000$, and $10,000 \mathrm{ng} / \mathrm{mL}$ in duplicate for each analytical run. The required amount of working stock was added to sufficient 
drug-free heparinized plasma to generate fresh quality control (QC) samples for each analytical run at concentrations of 10 (lower limit of quantitation; T-LLOQ), 30 (low range; T-LQC), 3000 (medium range; T-MQC), 9000 (high range; T-HQC), and 30,000 $\mathrm{ng} / \mathrm{mL}$ (10-fold dilution; T-DQC).

\subsubsection{Sample preparation}

Samples were thawed on wet ice and inverted 3 to 5 times to homogenize. Fifty microliters of $0.1 \mathrm{~N} \mathrm{NaOH}$ were added to $50 \mu \mathrm{L}$ of plasma calibrator aliquots, QC samples and unknown samples, then vortexed briefly to release CPT from NDCs. Following a 15 -minute incubation at room temperature, $200 \mu \mathrm{L}$ of $1 \%$ formic acid (FA) containing $250 \mathrm{ng} / \mathrm{mL}$ of SN-38 (internal standard) were added to lower $\mathrm{pH}$ and convert CPT to the lactone form. The resulting mixture was vortex-mixed, then centrifuged for 10 minutes at 13,200 rpm $(11,700 \mathrm{xg})$ in a mini-centrifuge. Approximately $250 \mu \mathrm{L}$ were removed and added to a solid-phase extraction (SPE) plate (Evolute Express ABN, Biotage; Charlotte, NC). Once on the SPE plate, the sample was washed with $1 \%$ aqueous methanol, and eluted in 100\% methanol. The eluent was dried down and reconstituted in 60/40 (v/v) 1\% FA (aq)/MeOH.

\subsection{Free CPT quantitation}

The purpose of this preparative method was to stabilize NLG207 NDCs using low $\mathrm{pH}$ conditions ${ }^{18}$ and quantitate only the free CPT, unbound to NLG207. 


\subsubsection{Preparation of stock solutions}

CPT Master Stock solutions were prepared via dissolution in DMSO at a concentration of $1 \mathrm{mg} / \mathrm{mL}$. The master stock solution was vortex mixed and briefly sonicated before being stored in glass vials at $-80^{\circ} \mathrm{C}$. Serial dilutions (working stocks) in DMSO were prepared from the master stock for the generation of calibration and QC samples and stored in glass vials at $-80^{\circ} \mathrm{C}$. SN-38 Master Stock was prepared as described for the total CPT quantitation.

Calibration standards were freshly prepared in drug-free human heparinized plasma at concentrations of $1,5,10,50,100,500$, and $1,000 \mathrm{ng} / \mathrm{mL}$ in duplicate for each analytical run. The required amount of working stock was added to sufficient drug-free heparinized plasma to generate fresh QC samples for each analytical run at concentrations of 1 (F-LLOQ), 3 (F-LQC), 300 (F-MQC), 900 (F-HQC), and 10,000 ng/mL (10-fold dilution; F-DQC).

\subsubsection{Sample preparation}

Similarly to the total CPT quantitation, samples were thawed on wet ice and inverted 3 to 5 times to homogenize. To one hundred microliters of plasma calibrator aliquots, QC samples, and unknown samples, $200 \mu \mathrm{L}$ of $1 \%$ formic acid containing 250 $\mathrm{ng} / \mathrm{mL}$ of SN-38 (internal standard) were added to stabilize NDCs, preventing release of CPT. The resulting mixture was vortexed, then centrifuged for 10 minutes at 13,200 rpm. Mixtures were processed via SPE, dried, and reconstituted as described in the total CPT quantitation procedure. 


\subsection{Instrument conditions}

Instrument conditions for both total and free CPT quantitation calibration methods were consistent. For each sample, five microliters were injected onto a Waters ACQUITY UPLC ${ }^{\circledR}$ system (Waters Corporation, Milford, MA) that included a binary pump, refrigerated autosampler $\left(6^{\circ} \mathrm{C}\right)$ and a temperature-controlled column compartment (room temperature). An Acquity UPLC BEH Shield RP18, 2.1x50mm, 1.7 $\mu \mathrm{m}$ column was used for chromatographic separation. The mobile phase comprised of A: $0.1 \% \mathrm{FA}$ in water, and $\mathrm{B}$ : methanol with a $0.4 \mathrm{~mL} / \mathrm{min}$ flow rate and a $3-$ minute total run time. A gradient of mobile phase B was implemented via a linear increase from $35 \%$ to $75 \%$ during the first 1.5 minutes, then a linear decrease back to $35 \%$ over 1.0 minute, then maintaining $35 \%$ for 0.5 minutes. The column eluent was directed into a SCIEX QTRAP5500® mass spectrometer (SCIEX, Framingham, MA). Selected reaction monitoring (SRM) in the positive ion mode was set to monitor CPT (lactone form) $(\mathrm{m} / \mathrm{z}$ $349.2 \rightarrow 305.2)$, and the internal standard SN-38 $(\mathrm{m} / z 393.2 \rightarrow 349.2)$ (Table S1). ${ }^{19}$ Peak integrations generated via SRM, and subsequent data analyses were performed using the Multi-Quant program in Analyst (SCIEX, Framingham, MA).

\subsection{Validation}

\subsubsection{Quadratic regression}

Calibration curves for total CPT and free CPT were constructed using leastsquares quadratic regression analysis of 7 point curves $(10$ to $10,000 \mathrm{ng} / \mathrm{mL}$ and 1 to $1,000 \mathrm{ng} / \mathrm{mL}$ for total CPT and free CPT, respectively); the peak area ratio of the analyte to the internal standard were plotted using $1 / x^{2}$ as a weighting factor $(x=$ ratio of the 
nominal analyte:IS concentration). Calibrator response functions and choice of regression analysis were investigated via correlation coefficient $(r)$ calculation and percent deviation $(\% \mathrm{DEV})$ determination for all calibrators.

\subsubsection{Trueness and precision}

The trueness (accuracy) and precision of CPT quantification was evaluated for each method on four separate days using separate sets of 5 different concentrations (LLOQ, LQC, MQC, HQC, and DQC 10-fold dilution). Blank plasma, internal standard only, and calibration standard samples were prepared in duplicate for each run; QC and LLOQ samples were prepared in replicates of five for each of the four days $(n=20)$. The percent difference between the mean observed concentration and the nominal concentration was used to determine trueness (\%DEV). Assay consistency and reproducibility were evaluated via calculation of within-run precision (WRP) and between-run precision (BRP), per the equations below:

$$
\begin{gathered}
W R P=100 \times \frac{\left(M S_{W I T}\right)^{0.5}}{G M} \\
B R P=100 \times \frac{\left(\frac{\left\{M S_{B E T}-M S_{W I T}\right\}}{n}\right)^{0.5}}{G M}
\end{gathered}
$$

With grand mean represented by GM, within-group mean squared by $\mathrm{MS}_{\mathrm{WIT}}$, betweengroup mean squared by $\mathrm{MS}_{\mathrm{BET}}$, and the number of repetitions by $n$. Bioanalytical evaluation allowed for $\pm 15 \%$ variability in trueness and precision, per FDA guidelines. ${ }^{20}$ 


\subsection{Stability}

The post-preparative stability at $4{ }^{\circ} \mathrm{C}$ of CPT and SN-38 in the 96 well injector plates in the autosampler pending analysis was assessed. For both the total CPT and free CPT quantitation methods, samples were injected and analyzed immediately following preparation, then re-injected and re-analyzed 24 hours after the initial analysis. The original values obtained from those samples were compared to the values obtained 24 hours later.

Stability tests were performed to analyze potential alterations in CPT concentrations in the context of NLG207 conjugation. CPT degradation was analyzed primarily via quantitation of total CPT and NDC stability was assessed via comparisons of free CPT (i.e. drug unbound to the NDC). The stability of NLG207 and CPT in human plasma was assessed following multiple freeze/thaw cycles. Samples were assayed in triplicate at four concentrations of NLG207 (30, 300, 3000, and $9000 \mathrm{ng} / \mathrm{mL}$, per CPT equivalent) following up to 4 freeze/thaw cycles at $-80^{\circ} \mathrm{C}$ (at least 12 hours for each freeze cycle). Samples were processed using both assay validation methods for the measurement of total and free CPT. In the same analytical run, a comparison of analyte concentrations following each storage period to analyte concentrations of freshly prepared samples was generated.

The stability of NLG207 NDCs in plasma at room temperature was evaluated, mimicking conditions of sample processing in the laboratory. NLG207 samples in human plasma at four different concentrations $(30,300,3000$, and $9000 \mathrm{ng} / \mathrm{mL}$, per CPT equivalent) were either extracted immediately (fresh) or incubated at room temperature, 
each in triplicate. Samples were processed using both assay methods for the measurement of total and free CPT. Following analysis from the same analytical run, the analyte concentrations after 1 or 2 hours at room temperature were compared to the concentrations of immediately extracted samples.

The stability of the NLG207 in whole blood, mimicking post-collection conditions on the inpatient unit, was assessed at room temperature or $4{ }^{\circ} \mathrm{C}$. NLG207 samples in whole blood at three different concentrations $(50,500$, and 5,000 $\mathrm{ng} / \mathrm{mL}$, per CPT equivalent), were either extracted immediately (fresh), kept at room temperature for 3 hours, or kept at $4^{\circ} \mathrm{C}$ for 24 hours, each in triplicate. Plasma was isolated via inversion of the sample 5 to 8 times and centrifugation for 5 minutes at 2,400 RPM at $4^{\circ} \mathrm{C}$. Samples were processed using both assay validation methods for the measurement of total and free CPT. The analyte concentrations (total and free CPT) for each of the incubation conditions were compared to the concentration of freshly prepared samples in the same analytical run.

\subsection{Extraction recovery and matrix effects}

CPT and SN-38 extraction efficiency, or recovery, following SPE was evaluated by comparison of analyte peak areas before and after placement on the SPE plate at multiple concentrations of CPT $(6,60,600$, and $6,000 \mathrm{ng} / \mathrm{mL}$; each $n=5)$, each with a constant concentration of SN-38 (250 ng/mL). Due to differences in sample volume between the total CPT and free CPT preparative methods, the concentrations listed are consistent with the amount of drug processed via the total CPT method; equivalent concentrations for the free CPT method would be halved (e.g. $6 \mathrm{ng} / \mathrm{mL}$ corresponds to 3 
$\mathrm{ng} / \mathrm{mL}$ for the free CPT method). To further characterize selective and matrix effects, CPT and SN-38 were spiked into five lots of plasma at each of the corresponding concentrations and assayed in quintuplet.

Plasma matrix effects on camptothecin and SN-38 mass spectrometric signals were evaluated via comparison of samples spiked in plasma to samples spiked in the reconstitution solution. Camptothecin and $\mathrm{SN}-38$ were spiked into the reconstitution solution $(60 / 40, \mathrm{v} / \mathrm{v} ; 1 \% \mathrm{FA}(\mathrm{aq}) / \mathrm{MeOH})$ at four concentrations of CPT $(6,60,600$, and $6,000 \mathrm{ng} / \mathrm{mL}$; each $n=3$ ), each with $250 \mathrm{ng} / \mathrm{mL}$ of SN-38. The peak areas of CPT and SN-38 were analyzed and compared to the same concentrations of drug spiked into 5 separate lots of plasma after extraction, removing potential discrepancies attributable to the extraction step.

\subsection{Clinical application}

The applicability of this method to measure CPT clinically was demonstrated via quantitation of serial plasma samples collected following administration of NLG207 12 $\mathrm{mg} / \mathrm{m}^{2}$ dose of via one-hour intravenous infusion. The plasma concentration vs time data was collected in 3 patients with metastatic castration-resistant prostate cancer (mCRPC) during and after the first dose of NLG207 prior to initiating enzalutamide as part of combination therapy (per NCT03531827). Blood was collected in $6 \mathrm{~mL}$ sodium heparin tubes (BD, Franklin Lakes, NJ) and immediately placed at $4{ }^{\circ} \mathrm{C}$ until time of processing (samples were processed at the study site). Plasma was isolated via inversion of the sample 5 to 8 times and centrifugation for 5 minutes at $2,400 \mathrm{RPM}$ at $4^{\circ} \mathrm{C}$, then stored at $-80^{\circ} \mathrm{C}$ until time of quantitation. Concentrations of total and free CPT were measured 
following simultaneous processing of the same samples using both quantitation methods. The pharmacokinetic data was compared to previously published data administering NLG207 as monotherapy to patients with advanced solid tumors. ${ }^{7}$ The clinical protocol was approved by the Institutional Review Board of the National Cancer Institute's Center for Cancer Research (Bethesda, MD).

Incurred sample reanalysis was conducted using plasma samples from the previously mentioned 3 patients with mCRPC. Plasma samples collected during the NLG207 intravenous infusion, at $\mathrm{C}_{\mathrm{MAX}}$, and during the elimination phase were included in the analysis $(n=27)$. Nine of the 27 samples were re-analyzed 6 months after original quantitation, whereas the remaining samples were re-analyzed 2 days after original quantitation. The repeated total CPT quantitation was compared to the original quantitation per sample (not pooled) using the following formula:

$$
\% \text { Difference }=\frac{(\text { Repeat }- \text { Original })}{\left(\frac{\text { Repeat }+ \text { Original }}{2}\right)} \times 100
$$

The repeated free CPT quantitation was also compared to the original quantitation, with free CPT being expressed as the "\% free" CPT in relation to the total CPT quantitation of the same plasma sample (i.e. \%free CPT $=[$ free $\mathrm{CPT}] /[$ total CPT $] \times 100)$. Since the free CPT quantitation is impacted by the CPT release from NDCs, differences in the free CPT quantitation were reported as the increase in \%free CPT. The percentage of samples within $\pm 20 \%$ of the mean (original and repeat) for the total CPT assessment and the percentage of samples with $<1 \%$ increase for $\%$ free CPT assessment were reported. 


\section{Results and discussion}

\subsection{Selectivity}

Figure S2 depicts several UPLC-MS/MS chromatograms, including drug-free extract (Fig. S2A), internal standard only (Fig. S2B), and LLOQ for both total and free CPT quantitation methods (10 ng/mL and $1 \mathrm{ng} / \mathrm{mL}$, Fig. S2C and S2D, respectively). Additionally, Figure 2 depicts the UPLC-MS/MS chromatograms of a clinical pharmacokinetic sample taken at the end of a $12 \mathrm{mg} / \mathrm{m}^{2}$ intravenous infusion of 60 minutes (total CPT and free CPT, Fig $\mathbf{2 A}$ and 2B, respectively); the LC method provided optimal separation of analyte and internal standard for both the total and free CPT procedures (Fig 2C and 2D, respectively). The retention times of CPT lactone and SN-38 were 1.46 and 1.66 min, respectively. For both sample processing methods, the elution following an organic gradient via UPLC yielded sharp, symmetrical peaks without interference from the plasma matrix.

\subsection{Validation}

The calibration standards, ranging from 10 to $10,000 \mathrm{ng} / \mathrm{mL}$ and 1 to 1,000 $\mathrm{ng} / \mathrm{mL}$ for total and free CPT quantitation respectively, were run in duplicate on each of four days $(n=8)$. The seven calibration standards for each quantitation method were accurate and precise (Table 1), with a mean $r^{2}$ value of $0.99639 \pm 0.00181$ and 0.99906 \pm 0.00058 for total and free CPT, respectively. It was initially attempted to quantitate the full range of 1 to $10,000 \mathrm{ng} / \mathrm{mL}$ for both sample preparation methods, but the final assay ranges were selected for increased between run consistency and more relevant clinical applicability. Reflective of differences in the range of the total and free CPT quantitation 
methods, four appropriate quality control (QC) samples, at low, medium, high, and tenfold diluted concentrations, and a LLOQ QC were run daily in quintuplet over four days $(n=20)$. The QC concentrations for both quantitation methods were accurate and precise, within the required $\leq 15 \%$ (Table 2). Of note, the quantitation of two samples for the total CPT validation (one calibration sample [50 ng/mL] and one QC sample [TLQC, $30 \mathrm{ng} / \mathrm{mL}]$ ), was omitted due to significant deviation from the nominal concentration resulting from inaccuracy in sample preparation.

\subsection{Stability}

The 24-hour post-preparative stability measurements resulted in minimal deviations compared with the initial run for both validation methods. Quality control measurements for both methods were also consistent with the initial run $(<6 \%$ change), enabling samples extracted from human plasma to be reanalyzed within 24 hours of preparation without significant degradation (Table S2). Additionally, the postpreparative stability permitted the simultaneous processing of samples using both total and free CPT methods, followed by sequential UPLC-MS/MS quantitation. For all subsequent stability analyses, the samples were processed for both total and free CPT quantitation simultaneously for more accurate and direct data comparisons.

Freeze/thaw stability of CPT and NLG207 NDCs in human plasma was shown following analysis of four freeze/thaw cycles at 4 different concentrations of NLG207 (concentration measured as total CPT equivalents; Table 3A). No significant degradation of small molecule CPT was indicated via comparisons of total CPT concentrations between freeze/thaw cycles $(<8 \%$ change). Standard processing of 
NLG207 $1 \mathrm{mg} / \mathrm{mL}$ (CPT equivalent) stocks stored in DPBS at $-80^{\circ} \mathrm{C}$ followed by immediate transfer to human plasma prior to processing consistently resulted in $3 \%$ [ 2.5 to $3.5 \%$ ] of CPT to be released from NLG207 NDCs, as indicated by the freeze/thaw dataset for all indicated concentrations. The noted free:total CPT ratio was not meant to be indicative of what is observed clinically, but rather served as an additional internal quality control for stability assessments. The free CPT concentrations and free:total CPT ratios following several freeze/thaws did not deviate significantly from freshly prepared samples, with $<13 \%$ and $<10 \%$ change, respectively (Table 3B and 3C).

Room temperature stability of NLG207 NDCs in human plasma was assessed at timepoints relevant to the timing of sample processing (Table 4). Total CPT concentrations were not affected by room temperature at one or two hours $(<10 \%$ change), indicating no significant degradation of CPT molecules. Free CPT concentrations were significantly impacted by 2 hours ( $>33 \%$ increase compared to fresh), with $>14 \%$ change measured for each concentration range after one hour. The data indicates limited NDC stability at room temperature and the importance of immediate sample processing following the thawing of a frozen sample, notably for accurate and precise free CPT quantitation.

The stability of NLG207 NDCs in whole blood prior to plasma separation was assessed at room temperature and $4{ }^{\circ} \mathrm{C}$ (Table 5). Following 3 hours at room temperature, the total CPT concentrations were not impacted $(<10 \%$ change), whereas the free CPT concentrations increased dramatically ( $>70 \%$ change). When stored at $4{ }^{\circ} \mathrm{C}$, the total and free CPT concentrations were not significantly altered after 24 hours 
$(<15 \%$ change for both total and free CPT). In summation, though CPT was stable in blood under both conditions, the NDCs were only stable at $4{ }^{\circ} \mathrm{C}$ after 24 hours and not at room temperature after 3 hours. The data confirms current inpatient hospital procedure, enabling samples to be stored on ice overnight and processed the subsequent morning without significant alterations in CPT concentration measurement.

\subsection{Extraction recovery and matrix effects}

The assessments of solid phase extraction efficiency, human plasma matrix effects, and overall process efficiency at 6,60,600, and $6000 \mathrm{ng} / \mathrm{mL} \mathrm{CPT} \mathrm{with}$ consistent concentrations of SN-38 are summarized in Table S3. The mean overall extraction recoveries of $\mathrm{CPT}$ and $\mathrm{SN}-38$, estimated via comparisons of mass spectrometric signal response for the molecules spiked into 5 different lots of human plasma pre- versus post-extraction, ranged from $75.99-85.35 \%$ and $86.04-89.19 \%$, respectively. When comparing the effects of human plasma to clean solution (process efficiency), analyte and IS peak areas between post-extraction plasma and mobile phase were reduced by $19.81-20.95 \%$ and $16.43-17.56 \%$, respectively, for analyte concentrations ranging from 6 to $600 \mathrm{ng} / \mathrm{mL}$; for the $6000 \mathrm{ng} / \mathrm{mL}$ concentrations, the analyte and IS signals were only reduced by $5.51 \%$ and $3.33 \%$, respectively. With respect to overall process efficiency, the mean ratio of analyte to IS recoveries was consistent across all concentration levels, however, with respect to extraction efficiency, mean analyte recovery increased with concentration while IS recovery remained relatively constant. The comparison of post-column analyte injection and blank plasma extract injection was consistent with negligible matrix effects. 


\subsection{Clinical application}

Figure 3 depicts the total and free CPT concentration vs. time curves for 3 patients with mCRPC who received a single dose of $12 \mathrm{mg} / \mathrm{m}^{2} \mathrm{NLG} 207$ via one-hour intravenous infusion (determined in human plasma). These results are in line with previous data reported from Phase I clinical evaluation of pharmacokinetic evaluation, ${ }^{7}$ suggesting the sufficiency of the developed analytical methods to detect CPT concentrations with clinically relevant sensitivity, enabling further evaluation of CPT plasma concentrations as part of ongoing Phase II evaluation of NLG207 treatment combinations. The clinical results presented here demonstrated the applicability of this assay in capturing the range of plasma concentrations achieved with clinical doses given. Further pharmacokinetic analyses will be published separately following the availability of more data.

The incurred sample reanalysis examined a total of 27 plasma samples collected from the previously mentioned patients with mCRPC. Comparisons of total CPT quantitation showed that $100 \%$ of samples were within $20 \%$ of the mean of original and repeat quantitation. Twenty-three of 27 plasma samples (85.2\%) reported less than $1 \%$ change increase in \%free CPT following repeat quantitation, with the greatest increase in \%free CPT reported as $1.64 \%$. Of the samples collected 6 months prior to reanalysis, 3 of 9 plasma samples (33\%) reported a $>1 \%$ change in \%free CPT, suggesting some caution with increased retention time of the plasma samples. Additionally, all samples with $>1 \%$ increase in \%free CPT were at later collection time points (e.g. 8, 12, and 24 hours post end of infusion). Results from the incurred sample reanalysis demonstrate reliability of the assay to provide consistent quantitation of NLG207 plasma samples. 


\section{Conclusions}

Outlined here are consistent and reliable analytical methods associated with quantitation of CPT in the context of NLG207, a promising therapeutic agent currently included as part of two combination treatments under investigation at the NCI. This method proved sensitive (T-LLOQ $10 \mathrm{ng} / \mathrm{mL}$, F-LLOQ $1 \mathrm{ng} / \mathrm{mL}$ ), selective, accurate, and precise for quantitating both NDC-bound and free CPT concentrations in clinically relevant ranges. The solid phase extraction proved to be efficient at recovering CPT ( $\sim 80 \%$ ), and approaches used to modify $\mathrm{pH}$ were sufficient for the exclusive detection of CPT lactone. This method demonstrated 24-hour post-preparative stability, a factor that enables the simultaneous processing and subsequent analysis of total and free CPT. NLG207 NDCs were found to be stable in plasma through four freeze/thaws and stable in whole blood at $4^{\circ} \mathrm{C}$ for 24 hours. The assay shows the importance of limiting sample exposure to room temperature, as the amount of CPT release from NLG207 was significant after 2 hours. Assessment of clinical application demonstrated the capability of this assay to produce results in line with previously published pharmacokinetic data. This assay is currently being applied to the pharmacokinetic analyses of two Phase II studies examining the treatment combinations of NLG207 and olaparib, and NLG207 and enzalutamide. 


\section{Acknowledgements}

This study was funded in part by the Intramural Research Program of the NIH, National Cancer Institute, and in part by a CRADA between the National Cancer Institute and NewLink Genetics (\#03089). This is US Government work. There are no restrictions on its use. The views expressed within this paper do not necessarily reflect those of the US Government. We thank the nursing staff of the National Cancer Institute and the fellows of the Medical Oncology Branch at the National Cancer Institute for their care of our patients. Most importantly, we appreciate the patients with cancer who enroll in investigational trials to advance the knowledge of this disease. 


\section{REFERENCES}

1. Li F, Jiang T, Li Q, Ling X. Camptothecin (CPT) and its derivatives are known to target topoisomerase I (Top1) as their mechanism of action: did we miss something in CPT analogue molecular targets for treating human disease such as cancer? Am J Cancer Res 2017;7:2350-94.

2. Herben VM, Ten Bokkel Huinink WW, Schellens JH, Beijnen JH. Clinical pharmacokinetics of camptothecin topoisomerase I inhibitors. Pharm World Sci 1998;20:161-72.

3. Thomas A, Pommier Y. Targeting Topoisomerase I in the Era of Precision Medicine. Clin Cancer Res 2019.

4. Clark AJ, Wiley DT, Zuckerman JE, et al. CRLX101 nanoparticles localize in human tumors and not in adjacent, nonneoplastic tissue after intravenous dosing. Proc Natl Acad Sci U S A 2016;113:3850-4.

5. Young C, Schluep T, Hwang J, Eliasof S. CRLX101 (formerly IT-101)-A Novel Nanopharmaceutical of Camptothecin in Clinical Development. Curr Bioact Compd 2011;7:8-14.

6. Davis ME. Design and development of IT-101, a cyclodextrin-containing polymer conjugate of camptothecin. Adv Drug Deliv Rev 2009;61:1189-92.

7. Weiss GJ, Chao J, Neidhart JD, et al. First-in-human phase 1/2a trial of CRLX101, a cyclodextrin-containing polymer-camptothecin nanopharmaceutical in patients with advanced solid tumor malignancies. Invest New Drugs 2013;31:986-1000. 8. Numbenjapon T, Wang J, Colcher D, et al. Preclinical results of camptothecinpolymer conjugate (IT-101) in multiple human lymphoma xenograft models. Clin Cancer Res 2009;15:4365-73.

9. Pham E, Birrer MJ, Eliasof S, et al. Translational impact of nanoparticle-drug conjugate CRLX101 with or without bevacizumab in advanced ovarian cancer. Clin Cancer Res 2015;21:808-18.

10. Conley SJ, Baker TL, Burnett JP, et al. CRLX101, an investigational camptothecin-containing nanoparticle-drug conjugate, targets cancer stem cells and impedes resistance to antiangiogenic therapy in mouse models of breast cancer. Breast Cancer Res Treat 2015;150:559-67.

11. Pham E, Yin M, Peters CG, et al. Preclinical Efficacy of Bevacizumab with CRLX101, an Investigational Nanoparticle-Drug Conjugate, in Treatment of Metastatic Triple-Negative Breast Cancer. Cancer Res 2016;76:4493-503.

12. Lin CJ, Lin YL, Luh F, Yen Y, Chen RM. Preclinical effects of CRLX101, an investigational camptothecin-containing nanoparticle drug conjugate, on treating glioblastoma multiforme via apoptosis and antiangiogenesis. Oncotarget 2016;7:4240821.

13. Schluep T, Hwang J, Cheng J, et al. Preclinical efficacy of the camptothecinpolymer conjugate IT-101 in multiple cancer models. Clin Cancer Res 2006;12:1606-14. 14. Chao J, Lin J, Frankel P, et al. Pilot trial of CRLX101 in patients with advanced, chemotherapy-refractory gastroesophageal cancer. J Gastrointest Oncol 2017;8:962-9. 15. Keefe SM, Hoffman-Censits J, Cohen RB, et al. Efficacy of the nanoparticledrug conjugate CRLX101 in combination with bevacizumab in metastatic renal cell 
carcinoma: results of an investigator-initiated phase I-IIa clinical trial. Ann Oncol 2016;27:1579-85.

16. Sanoff HK, Moon DH, Moore DT, et al. Phase I/II trial of nano-camptothecin CRLX101 with capecitabine and radiotherapy as neoadjuvant treatment for locally advanced rectal cancer. Nanomedicine 2019;18:189-95.

17. Voss MH, Hussain A, Vogelzang N, et al. A randomized phase II trial of CRLX101 in combination with bevacizumab versus standard of care in patients with advanced renal cell carcinoma. Ann Oncol 2017;28:2754-60.

18. Cheng J, Khin KT, Jensen GS, Liu A, Davis ME. Synthesis of linear, betacyclodextrin-based polymers and their camptothecin conjugates. Bioconjug Chem 2003; 14:1007-17.

19. Chen X, Peer CJ, Alfaro R, Tian T, Spencer SD, Figg WD. Quantification of irinotecan, SN38, and SN38G in human and porcine plasma by ultra high-performance liquid chromatography-tandem mass spectrometry and its application to hepatic chemoembolization. J Pharm Biomed Anal 2012;62:140-8.

20. Administration UFaD. Bioanalytical Method Validation Guidance for Industry. 2018. 


\section{FIGURES}

Figure 1. Subunit of NLG207 nanoparticle-drug conjugates

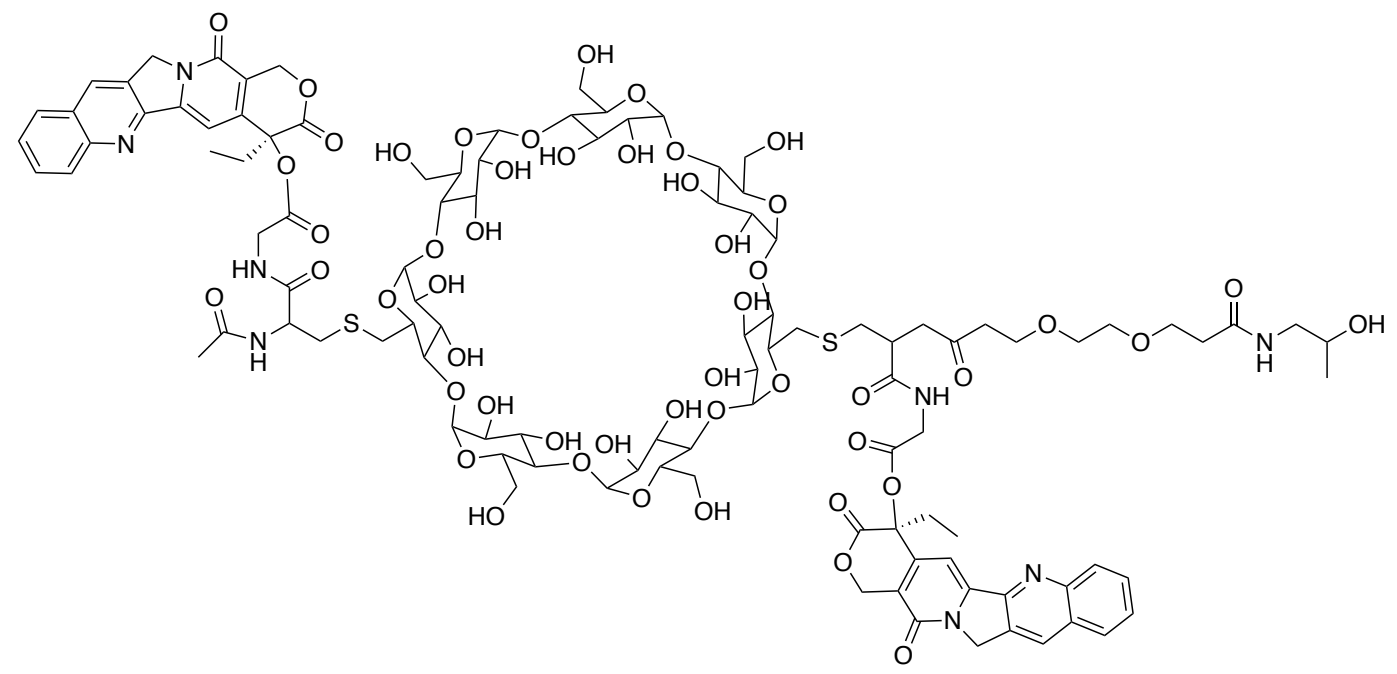


Figure 2. A clinical pharmacokinetic sample measured at the end of infusion of a $12 \mathrm{mg} / \mathrm{m}^{2}$ dose of NLG207, total (A \& C) and free CPT (B \& D). Chromatograms correspond to camptothecin lactone form (analyte), and SN-38 (internal standard).
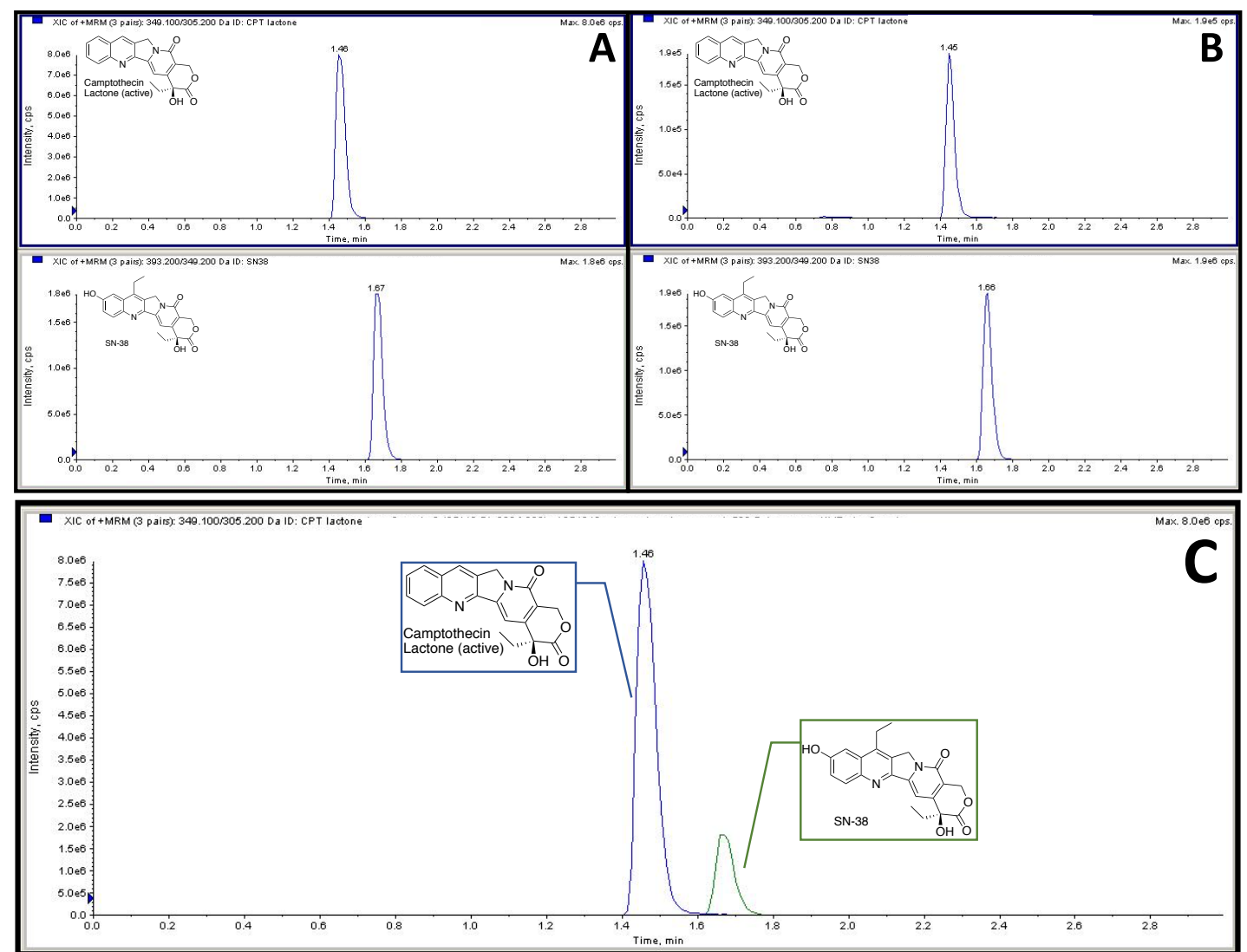

- XIC of +MRM (3 pairs): $349.100 / 305.200$ Da ID: CPT lactone

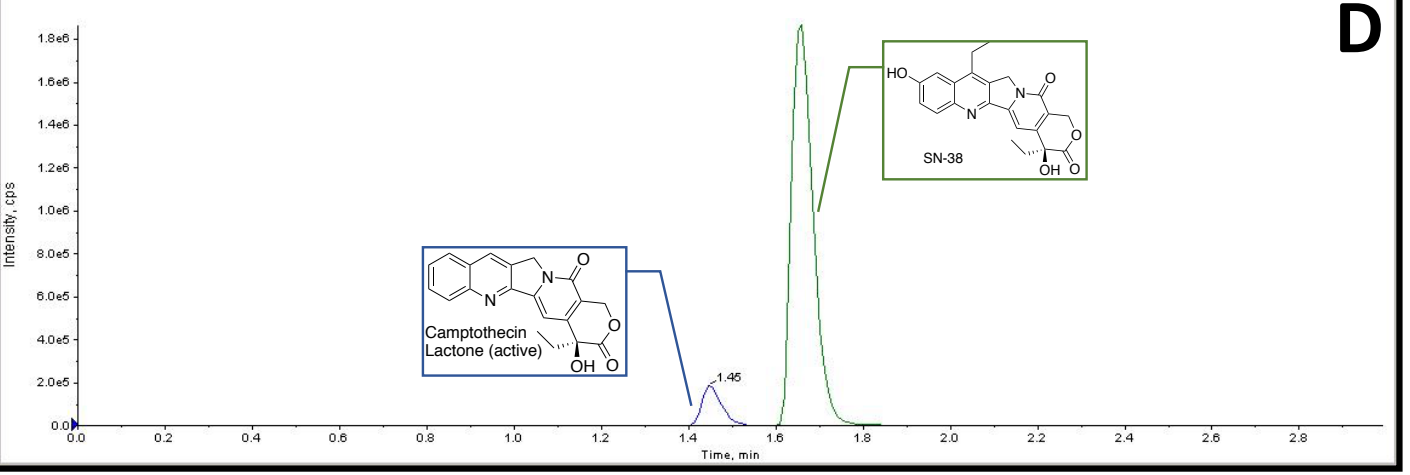


Figure 3. Total and free camptothecin (CPT) plasma concentration vs. time curves for three patients with $\mathrm{mCRPC}$ administered a one-hour intravenous infusion of NLG207 $12 \mathrm{mg} / \mathrm{m}^{2}$.

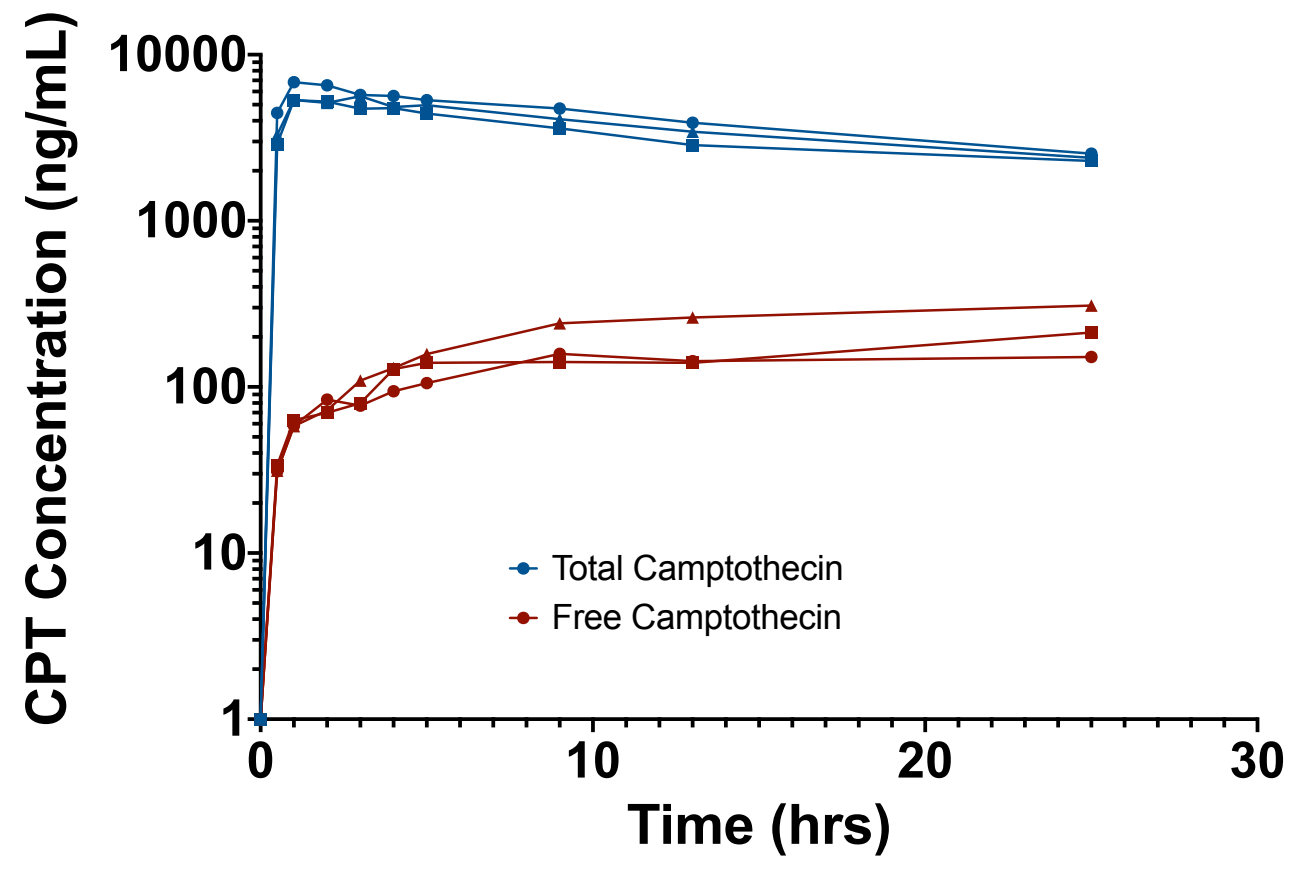




\section{TABLES}

Table 1. Calibration quadratic regression

\begin{tabular}{|l|l|l|l|l|l|}
\hline \multicolumn{1}{|c|}{$\begin{array}{c}\text { Nominal } \\
\mathbf{( n g / m L )}\end{array}$} & GM (ng/mL) & SD $\mathbf{( n g / m L )}$ & DEV (\%) & CV (\%) & $\boldsymbol{n}$ \\
\hline $\mathbf{1 0 . 0 0}$ & 10.01 & 0.32 & 0.14 & 3.25 & 8 \\
\hline $\mathbf{5 0 . 0 0}$ & 47.75 & 2.50 & -4.50 & 5.25 & 7 \\
\hline $\mathbf{1 0 0 . 0 0}$ & 94.10 & 2.65 & -5.90 & 2.82 & 8 \\
\hline $\mathbf{5 0 0 . 0 0}$ & 501.9 & 9.67 & 0.38 & 1.93 & 8 \\
\hline $\mathbf{1 0 0 0 . 0 0}$ & 1076 & 62.4 & 7.60 & 5.80 & 8 \\
\hline $\mathbf{5 0 0 0 . 0 0}$ & 5296 & 175 & 5.92 & 3.30 & 8 \\
\hline $\mathbf{1 0 0 0 0 . 0 0}$ & 9547 & 233 & -4.53 & 2.44 & 8 \\
\hline
\end{tabular}

\begin{tabular}{|l|l|l|l|l|l|}
\hline \multicolumn{1}{|c|}{$\begin{array}{c}\text { Nominal } \\
\mathbf{( n g / m L )}\end{array}$} & GM (ng/mL) & SD $(\mathbf{n g} / \mathbf{m L})$ & DEV (\%) & CV (\%) & $\boldsymbol{n}$ \\
\hline $\mathbf{1 . 0 0}$ & 1.01 & 0.03 & 0.50 & 3.19 & 8 \\
\hline $\mathbf{5 . 0 0}$ & 4.92 & 0.13 & -1.65 & 2.63 & 8 \\
\hline $\mathbf{1 0 . 0 0}$ & 9.92 & 0.11 & -0.78 & 1.16 & 8 \\
\hline $\mathbf{5 0 . 0 0}$ & 49.50 & 0.67 & -1.01 & 1.36 & 8 \\
\hline $\mathbf{1 0 0 . 0 0}$ & 101.5 & 1.83 & 1.53 & 1.80 & 8 \\
\hline $\mathbf{5 0 0 . 0 0}$ & 515.4 & 15.1 & 3.09 & 2.93 & 8 \\
\hline $\mathbf{1 0 0 0 . 0 0}$ & 983.6 & 14.1 & -1.64 & 1.43 & 8 \\
\hline
\end{tabular}

Abbreviations: GM, grand mean; SD, standard deviation; DEV (\%), relative deviation from nominal value; $\mathrm{CV}(\%)$, coefficient of variation; $n$, number of replicate observations within each validation run, i.e. for each concentration, two samples were run on four separate occasions (total of 8 samples at each concentration). 


\begin{tabular}{|c|c|c|c|c|c|c|c|}
\hline \multicolumn{8}{|c|}{ Total Camptothecin } \\
\hline $\begin{array}{l}\text { Nominal } \\
\text { (ng/mL) }\end{array}$ & $\begin{array}{l}\text { GM } \\
(\mathrm{ng} / \mathrm{mL})\end{array}$ & $\begin{array}{l}\text { SD } \\
(\mathrm{ng} / \mathrm{mL})\end{array}$ & $\begin{array}{l}\text { DEV } \\
(\%)\end{array}$ & $\begin{array}{l}\text { CV } \\
(\%)\end{array}$ & $\begin{array}{l}\text { WRP } \\
(\%)\end{array}$ & $\begin{array}{l}\text { BRP } \\
(\%)\end{array}$ & $\boldsymbol{n}$ \\
\hline 10 (T-LLOQ) & 10.34 & 0.87 & 3.43 & 8.44 & 4.54 & 8.01 & 20 \\
\hline 30 (T-LQC) & 33.03 & 1.71 & 10.09 & 5.18 & 3.39 & 4.29 & 19 \\
\hline $\begin{array}{l}3000 \text { (T- } \\
\text { MQC) }\end{array}$ & 3255 & 101 & 8.49 & 3.10 & 2.66 & 1.79 & 20 \\
\hline $\begin{array}{l}9000(T- \\
H Q C)\end{array}$ & 9249 & 386 & 2.77 & 4.17 & 3.73 & 2.10 & 20 \\
\hline $\begin{array}{l}30000(T- \\
\mathrm{DQC})\end{array}$ & 32510 & 585 & 8.36 & 1.80 & 1.88 & $-*$ & 20 \\
\hline
\end{tabular}

\begin{tabular}{|c|c|c|c|c|c|c|c|}
\hline \multicolumn{8}{|c|}{ Free Camptothecin } \\
\hline $\begin{array}{l}\text { Nominal } \\
(\mathrm{ng} / \mathrm{mL})\end{array}$ & $\begin{array}{l}\text { GM } \\
(\mathbf{n g} / \mathbf{m L})\end{array}$ & $\begin{array}{l}\text { SD } \\
\text { (ng/mL) }\end{array}$ & $\begin{array}{l}\text { DEV } \\
(\%)\end{array}$ & $\begin{array}{l}\text { CV } \\
(\%)\end{array}$ & $\begin{array}{l}\text { WRP } \\
(\%)\end{array}$ & $\begin{array}{l}\text { BRP } \\
(\%)\end{array}$ & $n$ \\
\hline $1(\mathrm{~F}-\mathrm{LLOQ})$ & 1.06 & 0.06 & 6.20 & 5.98 & 4.64 & 4.24 & 20 \\
\hline 3 (F-LQC) & 3.16 & 0.09 & 5.18 & 2.72 & 2.55 & 0.99 & 20 \\
\hline 300 (F-MQC) & 319.2 & 12.6 & 6.40 & 3.94 & 2.25 & 3.65 & 20 \\
\hline 900 (F-HQC) & 966.5 & 54.1 & 7.39 & 5.59 & 3.86 & 4.56 & 20 \\
\hline $\begin{array}{l}10000(F- \\
D Q C)\end{array}$ & 9703 & 404 & 7.81 & 4.17 & 2.65 & 3.62 & 20 \\
\hline
\end{tabular}

Abbreviations: GM, grand mean; SD, standard deviation; DEV (\%), relative deviation from nominal value; CV (\%), coefficient of variation; WRP (\%), within-run precision; BRP (\%), between-run precision; $n$, number of replicate observations within each validation run.

*- No additional variation was observed as a result of performing the assay in different runs. 
Table 3. Freeze/thaw stability of NLG207 NDCs in plasma, including measurements of total camptothecin concentrations (A), free camptothecin concentrations (B), and the calculated ratio of free to total camptothecin concentrations $(\mathbf{C})$.

\begin{tabular}{|c|c|c|c|c|c|c|c|c|}
\hline \multirow{3}{*}{\begin{tabular}{c|} 
A \\
$\begin{array}{c}\text { Free/Thaw } \\
\text { Cycle }\end{array}$
\end{tabular}} & \multicolumn{8}{|c|}{ Total Camptothecin } \\
\hline & \multicolumn{2}{|c|}{$30 \mathrm{ng} / \mathrm{mL}$} & \multicolumn{2}{|c|}{300 ng/mL } & \multicolumn{2}{|c|}{$3000 \mathrm{ng} / \mathrm{mL}$} & \multicolumn{2}{|c|}{$9000 \mathrm{ng} / \mathrm{mL}$} \\
\hline & $\begin{array}{c}\text { GM } \\
(\mathbf{n g} / \mathbf{m L})\end{array}$ & $\begin{array}{l}\text { DEV } \\
(\%)\end{array}$ & $\begin{array}{c}\text { GM } \\
(\mathbf{n g} / \mathbf{m L})\end{array}$ & $\begin{array}{l}\text { DEV } \\
(\%)\end{array}$ & $\begin{array}{c}\text { GM } \\
(\mathrm{ng} / \mathbf{m L})\end{array}$ & $\begin{array}{l}\text { DEV } \\
(\%)\end{array}$ & $\begin{array}{c}\text { GM } \\
(\mathbf{n g} / \mathbf{m L})\end{array}$ & $\begin{array}{l}\text { DEV } \\
(\%)\end{array}$ \\
\hline 0 (fresh) & 33.08 & - & 332.0 & - & 3503 & - & 9898 & - \\
\hline 1 & 32.88 & -0.6 & 315.1 & -5.1 & 3231 & -7.8 & 9470 & -4.3 \\
\hline 2 & 35.02 & 5.9 & 317.6 & -4.3 & 3296 & -5.9 & 9746 & -1.5 \\
\hline 3 & 35.34 & 6.8 & 335.1 & 1.0 & 3359 & -4.1 & 9696 & -2.0 \\
\hline 4 & 33.22 & 0.4 & 312.5 & -5.8 & 3272 & -6.6 & 9656 & -2.4 \\
\hline
\end{tabular}

\begin{tabular}{|c|c|c|c|c|c|c|c|c|}
\hline \multirow{3}{*}{$\begin{array}{c}\text { B } \\
\begin{array}{c}\text { Free/Thaw } \\
\text { Cycle }\end{array}\end{array}$} & \multicolumn{8}{|c|}{ Free Camptothecin } \\
\hline & \multicolumn{2}{|c|}{$30 \mathrm{ng} / \mathrm{mL}$} & \multicolumn{2}{|c|}{300 ng/mL } & \multicolumn{2}{|c|}{$3000 \mathrm{ng} / \mathrm{mL}$} & \multicolumn{2}{|c|}{$9000 \mathrm{ng} / \mathrm{mL}$} \\
\hline & $\begin{array}{c}\text { GM } \\
(\mathrm{ng} / \mathrm{mL})\end{array}$ & $\begin{array}{l}\text { DEV } \\
(\%)\end{array}$ & $\begin{array}{c}\text { GM } \\
(\mathrm{ng} / \mathrm{mL})\end{array}$ & $\begin{array}{l}\text { DEV } \\
(\%)\end{array}$ & $\begin{array}{c}\text { GM } \\
(\mathrm{ng} / \mathrm{mL})\end{array}$ & $\begin{array}{l}\text { DEV } \\
(\%)\end{array}$ & $\begin{array}{c}\text { GM } \\
(\mathrm{ng} / \mathrm{mL})\end{array}$ & $\begin{array}{l}\text { DEV } \\
(\%)\end{array}$ \\
\hline 0 (fresh) & 1.02 & - & 10.83 & - & 110.1 & - & 305.6 & - \\
\hline 1 & 1.08 & 5.6 & 10.68 & -1.4 & 104.7 & -4.9 & 321.9 & 5.3 \\
\hline 2 & 1.08 & 6.2 & 9.98 & -7.8 & 97.13 & -11.8 & 337.6 & 10.5 \\
\hline 3 & 1.09 & 7.2 & 10.12 & -6.6 & 97.05 & -11.9 & 300.5 & -1.7 \\
\hline 4 & 0.99 & -2.6 & 9.91 & -8.5 & 95.93 & -12.9 & 317.0 & 3.7 \\
\hline
\end{tabular}

\begin{tabular}{|c|c|c|c|c|c|c|c|c|}
\hline \multirow{3}{*}{$\begin{array}{c}\text { C } \\
\begin{array}{c}\text { Free/Thaw } \\
\text { Cycle }\end{array} \\
\end{array}$} & \multicolumn{8}{|c|}{ Ratio of Free:Total Camptothecin } \\
\hline & \multicolumn{2}{|c|}{$30 \mathrm{ng} / \mathrm{mL}$} & \multicolumn{2}{|c|}{$300 \mathrm{ng} / \mathrm{mL}$} & \multicolumn{2}{|c|}{$3000 \mathrm{ng} / \mathrm{mL}$} & \multicolumn{2}{|c|}{$9000 \mathrm{ng} / \mathrm{mL}$} \\
\hline & $\begin{array}{c}\text { \% Free } \\
\text { CPT }\end{array}$ & $\begin{array}{l}\text { DEV } \\
(\%)\end{array}$ & $\begin{array}{c}\text { \% Free } \\
\text { CPT }\end{array}$ & $\begin{array}{l}\text { DEV } \\
(\%)\end{array}$ & $\begin{array}{c}\text { \% Free } \\
\text { CPT }\end{array}$ & $\begin{array}{l}\text { DEV } \\
(\%)\end{array}$ & $\begin{array}{c}\text { \% Free } \\
\text { CPT }\end{array}$ & $\begin{array}{l}\text { DEV } \\
(\%)\end{array}$ \\
\hline 0 (fresh) & 3.18 & - & 3.37 & - & 3.24 & - & 3.19 & - \\
\hline 1 & 3.27 & 2.9 & 3.39 & 6.5 & 3.24 & 1.9 & 3.40 & 6.8 \\
\hline 2 & 3.09 & -2.8 & 3.14 & -1.2 & 2.95 & -7.4 & 3.46 & 8.9 \\
\hline 3 & 3.09 & -2.8 & 3.02 & -5.1 & 2.89 & -9.2 & 3.10 & -2.6 \\
\hline 4 & 2.99 & -6.0 & 3.17 & -0.3 & 2.93 & -7.9 & 3.28 & 3.1 \\
\hline
\end{tabular}

Abbreviations: GM, grand mean; DEV (\%), relative deviation from fresh. 
Table 4. Bench-top plasma stability of NLG207 nanoparticles at room temperature

\begin{tabular}{|c|c|c|c|c|c|}
\hline \multirow{2}{*}{\begin{tabular}{l}
\multicolumn{1}{c}{ Nominal } \\
-Total CPT \\
-[Free CPT]*
\end{tabular}} & \multirow{2}{*}{$\begin{array}{c}\text { Immediately } \\
\text { After } \\
\text { Preparation } \\
\text { GM }(\mathrm{ng} / \mathrm{mL})\end{array}$} & \multicolumn{2}{|c|}{$\begin{array}{l}\text { One Hour Post } \\
\text { Preparation }\end{array}$} & \multicolumn{2}{|c|}{$\begin{array}{l}\text { Two Hours Post } \\
\text { Preparation }\end{array}$} \\
\hline & & $\begin{array}{c}\text { GM } \\
(\mathrm{ng} / \mathrm{mL})\end{array}$ & $\begin{array}{l}\text { MC } \\
(\%)\end{array}$ & $\begin{array}{c}\text { GM } \\
(\mathrm{ng} / \mathrm{mL})\end{array}$ & $\begin{array}{l}\text { MC } \\
(\%) \\
\end{array}$ \\
\hline \multirow{2}{*}{$\begin{array}{l}30 \mathrm{ng} / \mathrm{mL} \\
{[1.0 \mathrm{ng} / \mathrm{mL}]}\end{array}$} & 32.07 & 35.03 & 9.2 & 34.05 & 6.2 \\
\hline & 1.01 & 1.18 & 17.6 & 1.35 & 33.8 \\
\hline \multirow{2}{*}{$\begin{array}{l}300 \mathrm{ng} / \mathrm{mL} \\
{[9 \mathrm{ng} / \mathrm{mL}]}\end{array}$} & 342.7 & 330.29 & -3.6 & 337.0 & -1.7 \\
\hline & 9.34 & 10.91 & 16.8 & 13.19 & 41.2 \\
\hline \multirow{2}{*}{$\begin{array}{l}3000 \mathrm{ng} / \mathrm{mL} \\
{[90 \mathrm{ng} / \mathrm{mL}]}\end{array}$} & 3282 & 3449 & 5.1 & 3496 & 6.5 \\
\hline & 90.28 & 110.1 & 22.0 & 130.7 & 44.8 \\
\hline \multirow{2}{*}{$\begin{array}{l}9000 \mathrm{ng} / \mathrm{mL} \\
{[270} \\
\mathrm{ng} / \mathrm{mL}]\end{array}$} & 9207 & 9237 & 0.3 & 9395 & 2.0 \\
\hline & 300.7 & 345.0 & 14.7 & 422.1 & 40.4 \\
\hline
\end{tabular}

Abbreviations: GM, grand mean; MC, mean change from fresh.

*- Based on the totality of data, approximately $3 \%$ [2.5 to 3.5\%] of total CPT from NLG207 NDCs is present as free CPT under standard conditions. The nominal concentrations of free CPT listed are 3\% of the nominal total CPT concentrations. For the $30 \mathrm{ng} / \mathrm{mL}$ total CPT concentration range, the nominal free CPT concentration is rounded up to $1.0 \mathrm{ng} / \mathrm{mL}(3.33 \%$ of total CPT) to reflect the lower limit of quantitation. 
Table 5. Pre-processing blood stability of NLG207 nanoparticles at room temperature and at $4^{\circ} \mathrm{C}$

\begin{tabular}{|c|c|c|c|c|c|}
\hline \multirow{2}{*}{$\begin{array}{l}\text { Nominal (CPT } \\
\text { Equivalents) } \\
\text {-Total CPT } \\
\text {-[Free CPT]* }\end{array}$} & \multirow{2}{*}{$\begin{array}{c}\text { Immediately } \\
\text { After } \\
\text { Preparation } \\
\text { GM (ng/mL) }\end{array}$} & \multicolumn{2}{|c|}{$\begin{array}{l}3 \text { hours at Room } \\
\text { Temperature }\end{array}$} & \multicolumn{2}{|c|}{24 Hours at $4^{\circ} \mathrm{C}$} \\
\hline & & $\begin{array}{c}\text { GM } \\
(\mathrm{ng} / \mathrm{mL})\end{array}$ & $\begin{array}{l}\text { MC } \\
(\%)\end{array}$ & $\begin{array}{c}\text { GM } \\
(\mathrm{ng} / \mathrm{mL})\end{array}$ & $\begin{array}{c}\text { MC } \\
(\%)\end{array}$ \\
\hline \multirow{2}{*}{$\begin{array}{l}50 \mathrm{ng} / \mathrm{mL} \\
{[1.5 \mathrm{ng} / \mathrm{mL}]}\end{array}$} & 49.64 & 51.64 & 4.0 & 53.23 & 7.2 \\
\hline & 1.39 & 2.58 & 86.0 & 1.40 & 1.0 \\
\hline \multirow{2}{*}{$\begin{array}{l}500 \mathrm{ng} / \mathrm{mL} \\
{[15 \mathrm{ng} / \mathrm{mL}]}\end{array}$} & 554.4 & 579.7 & 4.6 & 595.3 & 7.4 \\
\hline & 16.83 & 28.74 & 70.8 & 14.40 & -14.4 \\
\hline \multirow{2}{*}{$\begin{array}{l}5000 \mathrm{ng} / \mathrm{mL} \\
{[150 \mathrm{ng} / \mathrm{mL}]}\end{array}$} & 5240 & 5549 & 4.7 & 5035 & -3.9 \\
\hline & 152.5 & 300.0 & 96.7 & 139.4 & -8.6 \\
\hline
\end{tabular}

Abbreviations: GM, grand mean; MC, mean change from fresh.

*- Based on the totality of data, approximately $3 \%$ [within 2.5 to $3.5 \%$ ] of total CPT from NLG207 NDCs is present as free CPT under standard conditions. The nominal concentrations of free CPT listed are $3 \%$ of the nominal total CPT concentrations. 
Figure S1. Structures of camptothecin (lactone and carboxylate forms) and internal standard, SN-38.
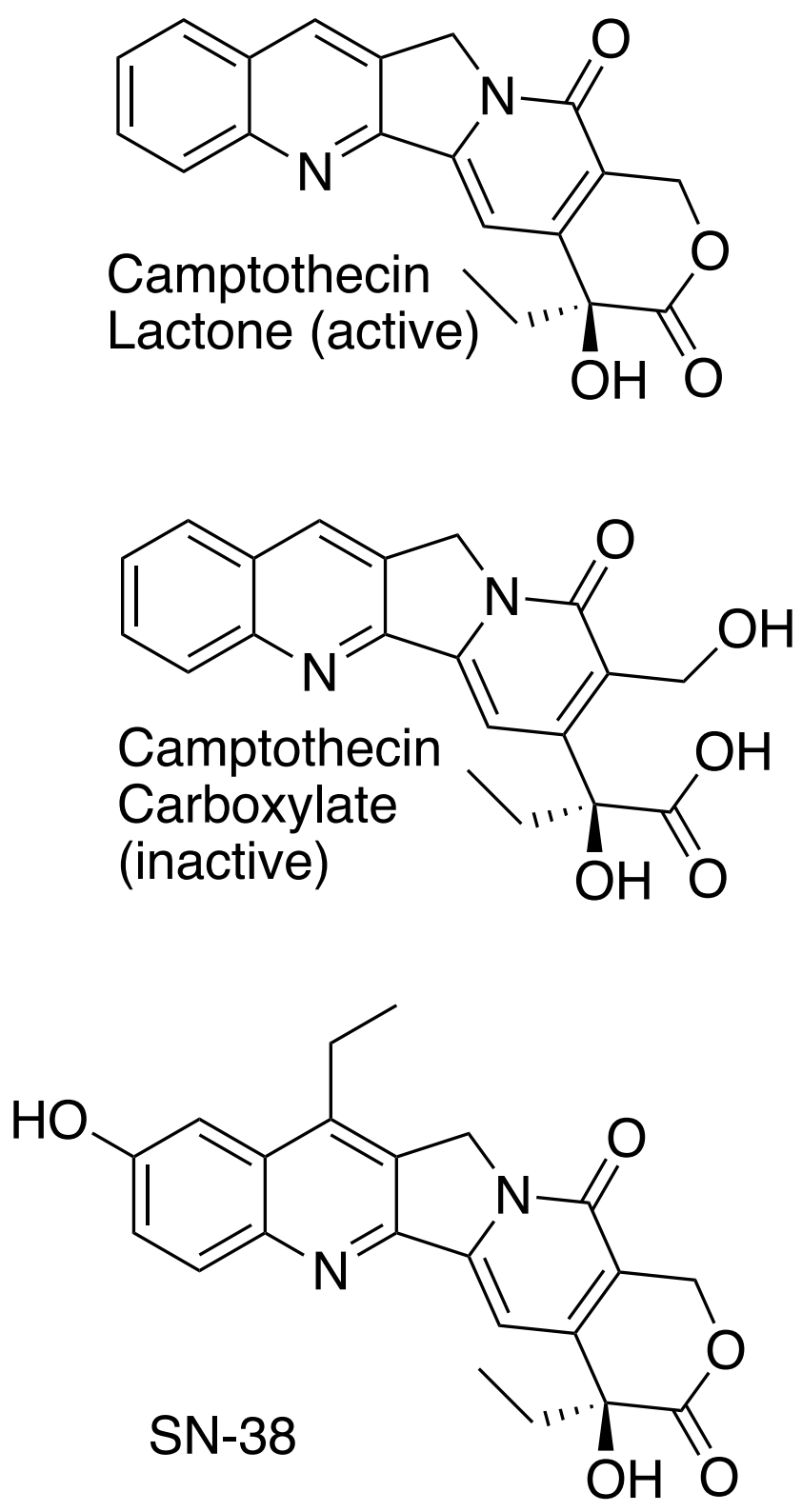
Figure S2: Chromatograms of (A) a blank plasma extract, (B) internal standard only extract, the lower limits of quantitation (LLOQ) for total (C) and free CPT (D).
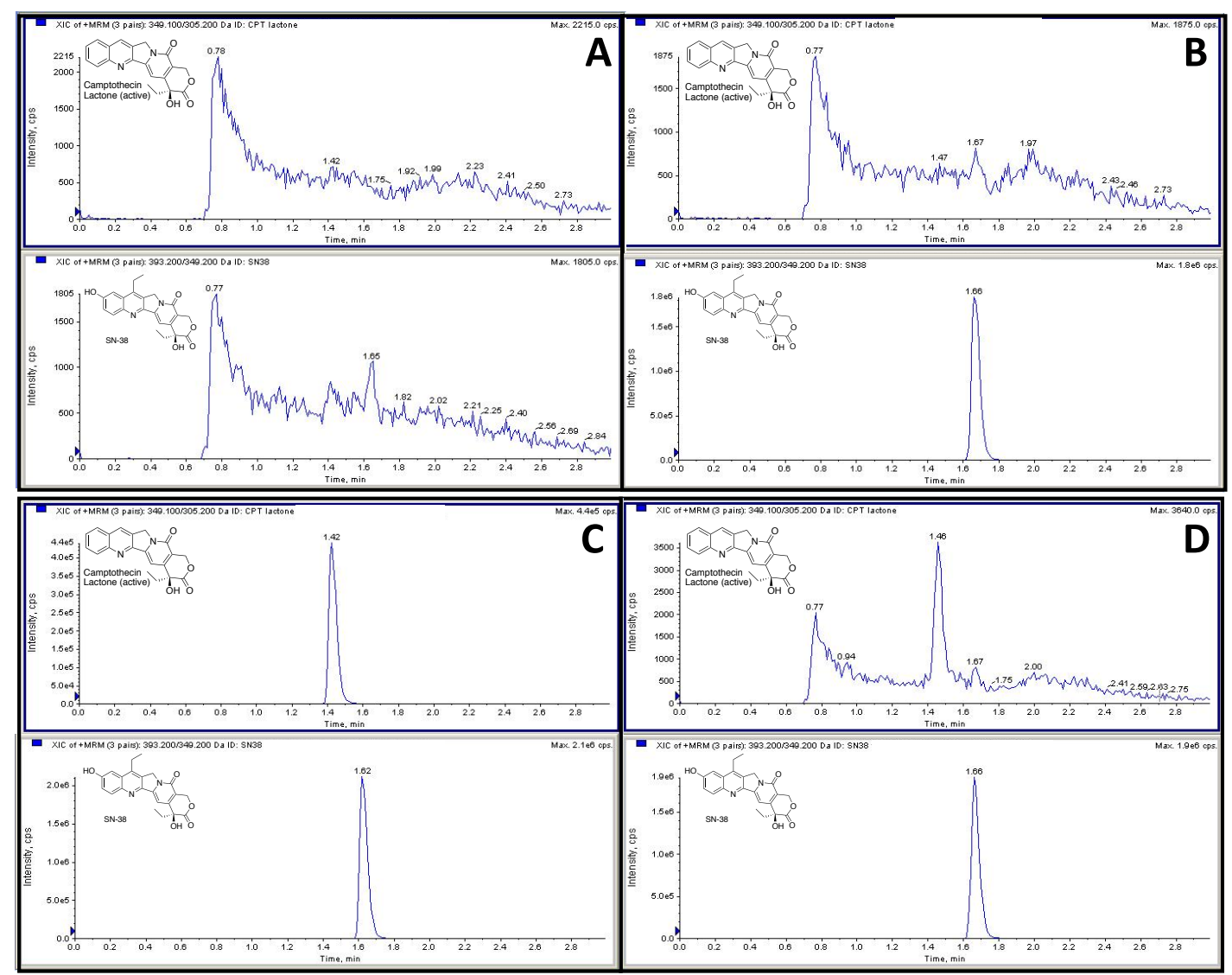
Table S1. Settings for selected reaction monitoring (SRM)

\begin{tabular}{|c|c|c|c|c|c|c|}
\hline Compound Name & $\begin{array}{c}\text { Parent } \\
(\mathbf{m} / \mathbf{z})\end{array}$ & $\begin{array}{c}\text { Daughter } \\
(\mathbf{m} / \mathbf{z})\end{array}$ & DP & EP & $\begin{array}{c}\text { CE } \\
(\mathbf{e V})\end{array}$ & CXP \\
\hline Camptothecin (lactone) & 349.2 & 305.2 & 70 & 11 & 32 & 5.6 \\
\hline SN-38 & 393.2 & 349.2 & 70 & 3.6 & 35.2 & 10 \\
\hline
\end{tabular}

Abbreviations: DP, Declustering Potential; EP, Entrance Potential; CE, Collision Energy; CXP, Collision Cell Exit Potential. 
Table S2. Post-preparative stability of quality controls

\begin{tabular}{|c|c|c|c|}
\hline & \multicolumn{3}{|c|}{ Total Camptothecin } \\
\hline $\begin{array}{l}\text { Nominal } \\
(\mathrm{ng} / \mathrm{mL})\end{array}$ & $\begin{array}{c}\text { Immediately } \\
\text { after Preparation } \\
\text { [GM }(\mathrm{ng} / \mathrm{mL})]\end{array}$ & $\begin{array}{l}24 \text { Hours after } \\
\text { Preparation } \\
\text { [GM }(\mathrm{ng} / \mathrm{mL})]\end{array}$ & $\begin{array}{c}\text { Mean Change } \\
\text { after } 24 \text { hours } \\
(\%)\end{array}$ \\
\hline 10 (T-LLOQ) & 10.21 & 9.69 & -5.0 \\
\hline 30 (T-LQC) & 33.65 & 34.36 & 2.1 \\
\hline 3000 (T-MQC) & 3302 & 3303 & $<0.1$ \\
\hline 9000 (T-HQC) & 9485 & 10050 & 6.0 \\
\hline 30000 (T-DQC) & 32620 & 33250 & 1.9 \\
\hline
\end{tabular}

\begin{tabular}{|c|c|c|c|}
\hline \multirow[b]{2}{*}{$\begin{array}{l}\text { Nominal } \\
(\mathrm{ng} / \mathrm{mL})\end{array}$} & \multicolumn{3}{|c|}{ Free Camptothecin } \\
\hline & $\begin{array}{c}\text { Immediately } \\
\text { after Preparation } \\
{[\mathrm{GM}(\mathrm{ng} / \mathrm{mL})]}\end{array}$ & $\begin{array}{l}24 \text { Hours after } \\
\text { Preparation } \\
\text { [GM }(\mathrm{ng} / \mathrm{mL})]\end{array}$ & $\begin{array}{c}\text { Mean Change } \\
\text { after } 24 \text { hours } \\
(\%)\end{array}$ \\
\hline 1 (F-LLOQ) & 1.08 & 1.04 & -3.7 \\
\hline 3 (F-LQC) & 3.20 & 3.14 & -2.1 \\
\hline 300 (F-MQC) & 327.9 & 320.9 & -2.1 \\
\hline 900 (F-HQC) & 903.2 & 911.4 & 0.9 \\
\hline 10000 (F-DQC) & 9168 & 9177 & 0.1 \\
\hline
\end{tabular}

Abbreviations: GM, grand mean 
Table S3. Extraction recovery and matrix effects

\begin{tabular}{|c|c|c|c|c|c|c|}
\cline { 2 - 7 } \multicolumn{1}{c|}{} & \multicolumn{2}{c|}{$\begin{array}{c}\text { Extraction } \\
\text { Efficiency (\%) }\end{array}$} & \multicolumn{2}{c|}{$\begin{array}{c}\text { Matrix Effects } \\
(\%)\end{array}$} & \multicolumn{2}{c|}{$\begin{array}{c}\text { Process } \\
\text { Efficiency (\%) }\end{array}$} \\
\hline $\begin{array}{c}\text { Analyte } \\
\text { Concentration* }\end{array}$ & $\mathbf{C P T}$ & $\mathbf{S N - 3 8}$ & $\mathbf{C P T}$ & $\mathbf{S N - 3 8}$ & $\mathbf{C P T}$ & $\mathbf{S N - 3 8}$ \\
\hline $\mathbf{6} \mathbf{~ n g / m L}$ & 75.99 & 86.04 & 104.02 & 95.81 & 79.05 & 82.44 \\
\hline $\mathbf{6 0} \mathbf{~ n g / m L}$ & 79.44 & 89.19 & 100.38 & 93.80 & 79.74 & 83.66 \\
\hline $\mathbf{6 0 0} \mathbf{~ n g / m L}$ & 82.04 & 88.84 & 97.74 & 94.07 & 80.19 & 83.57 \\
\hline $\mathbf{6 0 0 0} \mathbf{~ n g / m L}$ & 85.35 & 88.86 & 110.72 & 108.79 & 94.49 & 96.67 \\
\hline
\end{tabular}

Abbreviation: CPT, Camptothecin.

*- For purposes of this assessment, analyte concentration corresponds to the amount of drug used in the "Total CPT validation". The volume of sample used for the Free CPT validation is doubled, thus if viewing this assessment in the context of measuring free CPT, the analyte concentrations would correspond to half the concentration (e.g. 6 $\mathrm{ng} / \mathrm{mL}$ in the "total CPT" validation corresponds to $3 \mathrm{ng} / \mathrm{mL}$ of "free CPT" validation). The concentration of SN-38 (internal standard) was consistent for all samples throughout the assessment. 


\section{Chapter 6}

\section{Population Pharmacokinetic Analysis of Nanoparticle-bound and Free Camptothecin after Administration of NLG207 in Adults with Advanced Solid Tumors}

Keith T. Schmidt ${ }^{1}$, Alwin D. R. Huitema ${ }^{2,3}$, Thomas P. C. Dorlo ${ }^{2}$, Cody J. Peer ${ }^{1}$, Lisa M. Cordes $^{4}$ Linda Sciuto ${ }^{5}$, Susan Wroblewski ${ }^{4}$, Yves Pommier 5 , Ravi A. Madan ${ }^{4}$, Anish Thomas $^{5}$, and William D. Figg ${ }^{1,4}$

${ }^{1}$ Clinical Pharmacology Program, Center for Cancer Research, National Cancer Institute, National Institutes of Health, Bethesda, MD, USA

${ }^{2}$ Dept. Pharmacy \& Pharmacology, Netherlands Cancer Institute, Amsterdam, The Netherlands

${ }^{3}$ Department of Clinical Pharmacy, University Medical Center Utrecht, Utrecht University, Utrecht, The Netherlands

${ }^{4}$ Genitourinary Malignancies Branch, Center for Cancer Research, National Cancer Institute, National Institutes of Health, Bethesda, MD, USA

${ }^{5}$ Developmental Therapeutics Branch, Center for Cancer Research, National Cancer Institute, National Institutes of Health, Bethesda, MD, USA

Cancer Chemother and Pharmacol. In Press 

ABSTRACT
Purpose: NLG207 (formerly CRLX101) is a nanoparticle-drug conjugate (NDC) of the potent topoisomerase I inhibitor, camptothecin (CPT). The present study sought to characterize the complex pharmacokinetics (PK) of NLG207 and better describe CPT release from nanoparticles using a population PK (popPK) model.

Methods: From 27 patients enrolled on two phase II clinical trials (NCT02769962 and NCT03531827), dense sampling was performed up to 48 hours post-administration of NLG207 during cycle one and six of treatment; samples were also collected at $\sim 360$ hours post-dose. Conjugated and free CPT concentrations were quantified from each sample, resulting in 477 observations to build a popPK model using non-linear mixedeffects modelling.

Results: The PK of NLG207 was characterized by combining two linear twocompartment models with first-order kinetics each to describe nanoparticle-bound (conjugated) and free CPT. Allometric scaling based on body weight provided the best body-size descriptor for all PK parameters. The typical volumes of distribution of the conjugated CPT central and free CPT central compartments were $3.16 \mathrm{~L}$ (BSV CV\%; $18.1 \%)$ and $21.1 \mathrm{~L}(\mathrm{CV} \%$; 79.8\%), respectively. CPT release from the nanoparticle formulation was characterized via an initial rapid clearance of $5.71 \mathrm{~L} / \mathrm{h}(\mathrm{CV} \% ; 62.6 \%)$, which decreased via first-order decay (estimated half-life of $0.307 \mathrm{~h}$ ) to the steady-state value of $0.0988 \mathrm{~L} / \mathrm{h}(\mathrm{CV} \% ; 33.5 \%)$ by $\sim 4$ hours after end of infusion. Renal clearance of free CPT was $0.874 \mathrm{~L} / \mathrm{h}(\mathrm{CV} \% ; 42.2 \%)$.

Conclusion: The popPK model confirmed nanoparticle behavior of conjugated CPT and mechanistically characterized CPT release from NLG207. The current analysis provides 
a strong foundation for future study as a potential predictive tool in ongoing NLG207 clinical trials. 


\section{INTRODUCTION}

NLG207 (formerly CRLX101) is a nanoparticle-drug conjugate (NDC) of camptothecin (CPT), a potent topoisomerase I (TOP1) inhibitor, designed to overcome the poor physicochemical and pharmacokinetic (PK) properties (e.g. pH labile activation, limited plasma solubility) associated with small molecule delivery to tumors. ${ }^{1}$ Despite significant activity in preclinical models, CPT showed minimal antitumor activity in a clinical setting, and was associated with significant toxicities, primarily myelosuppression and hemorrhagic cystitis. ${ }^{2-5}$ Current FDA-approved TOP1 inhibitors (e.g. irinotecan, topotecan) are derived from the CPT backbone with improved solubility, but are still associated with sub-optimal PK and clinically significant toxicity. ${ }^{6}$ NLG207 consists of co-polymer units of $\beta$-cyclodextrin linked to adjacent units of poly-ethylene glycol (PEG), with conjugation of CPT via carboxylate esters. In aqueous solution, 4-5 co-polymer strands readily interact and fold into nanoparticles of 20-40 $\mathrm{nm}$ in diameter, providing a soluble delivery vehicle with increased plasma retention time..$^{7,8}$ The properties of the nanoparticle reduce glomerular filtration, mononuclear phagocyte system (MPS) interactions, and uptake into healthy tissues, while enabling passive selection into tumor via an enhanced permeation and retention (EPR) effect driven by size exclusion. ${ }^{8}$ Once deposited into tumors, CPT is slowly released from the NDCs via $\mathrm{pH}$-dependent hydrolysis of the carboxylate esters and subsequently renally cleared, limiting exposure of the small molecule in the systemic circulation. $^{7}$

NLG207 is well-tolerated, with over 300 patients spanning multiple tumor histologies (e.g. non-small cell lung cancer, advanced ovarian cancer, metastatic renal 
cell carcinoma) receiving at least one dose via numerous clinical trials. ${ }^{9-14}$ In the majority of studies, NLG207 has been administered at a dose of $15 \mathrm{mg} / \mathrm{m}^{2}$ via intravenous infusion every two weeks, with once weekly and $12 \mathrm{mg} / \mathrm{m}^{2}$ dosing strategies also investigated. ${ }^{1}$ TOP1 inhibitors also have HIF-1 $\alpha$ modulating effects, ${ }^{15}$ leading to evaluation of NLG207 as both monotherapy, and in combination with either a secondary chemotherapeutic agent (e.g. paclitaxel) or anti-angiogenic agent (e.g. bevacizumab). ${ }^{10,13,16}$ Ongoing studies at the National Cancer Institute (NCI) are evaluating the efficacy of NLG207 in combination with the PARP inhibitor, olaparib, in small-cell lung cancer, urothelial carcinoma, and prostate cancer, and with the androgen receptor antagonist, enzalutamide, in prostate cancer (NCT02769962 and NCT03531827, respectively).

Despite improvements in CPT delivery, commonly reported adverse events (AEs) of NLG207 included fatigue, myelosuppression, and, notably, bladder-associated toxicity..$^{9,10,12-14}$ In response to hemorrhagic cystitis occurring early in phase I dose escalation, hydration strategies have been successfully implemented pre and post NLG207 infusion to dilute accumulated CPT concentrations in the bladder; however, a low frequency of subsequent patients receiving NLG207 still reported low-grade cystitis, dysuria, and hematuria. ${ }^{14}$ Variable urinary excretion of CPT during the first 48 hours post-infusion has also been reported, with a mean of $21 \%$ of the total NLG207 dose eliminated in urine, a majority of which is eliminated in the first 8 hours. ${ }^{14}$ Previous non-compartmental analyses of NLG207 PK, which have analyzed nanoparticle-bound (conjugated) and free CPT independently, did not adequately 
address the release of CPT from the formulation, a critical component to understanding free CPT exposure and potential correlation to clinically observed toxicity.

The purpose of the present population pharmacokinetic (popPK) analysis was to 1) better understand CPT release from the NLG207 NDCs following administration, and 2) fully characterize the complex PK of conjugated and free CPT via a harmonized model.

\section{METHODS}

\section{Patients and Study Design}

NLG207 PK were evaluated using a population of patients with advanced solid tumors enrolled in two clinical studies at the National Cancer Institute analyzing NLG207 in combination with either olaparib or enzalutamide (NCT02769962 and NCT03531827, respectively). The PK analyses of these studies were designed to address potential drug-drug interactions associated with co-administration of olaparib or enzaluamide; the popPK analyses are a post-hoc study objective. Both studies were conducted according to IRB-approved protocols and all patients provided written informed consent.

NLG207 was administered via $12 \mathrm{mg} / \mathrm{m}^{2}$ intravenous infusions over one or two hours every two weeks, with the potential for incremental dose changes (e.g. decrease to 9 or $6 \mathrm{mg} / \mathrm{m}^{2}$ ) in subsequent cycles depending on the study and tolerability. Blood samples were collected during cycle 1 for both studies without co-administration of either olaparib or enzalutamide. Collection of samples occurred up to 48 hours (predose, mid-infusion [MI], end-of infusion [EOI], 1, 2, 12, 24 and $48 \mathrm{~h}$ post-EOI) and up 
to 24 hours (pre-dose, MI, EOI, 1, 2, 3, 4, 8, 12, and 24 h post-EOI) for NCT02769962 and NCT03531827, respectively. For NCT02769962, olaparib was only administered 48 hours after completion of the NLG207 infusion and administration was discontinued 48 hours prior to subsequent NLG207 infusions, reducing the likelihood of compounded myelosuppression from both agents in combination; ${ }^{17}$ additional samples were collected $\sim 336$ to $384 \mathrm{~h}$ (14 to 16 days) post-EOI and during cycle 6 using the same timepoints for patients remaining on therapy. Data collected on NCT03531827 only includes samples from cycle 1. Of note, NLG207 on NCT02776992 was administered at a slower rate during the first 10-15 minutes infusion, before increasing the rate to complete the infusion over the course of one or two hours, whereas NCT03531827 administered NLG207 at a constant infusion rate over the course of one hour.

\section{Pharmacokinetic Samples and Analyses}

Blood samples, collected in sodium heparin treated tubes (green top tubes, BD Biosciences), were processed into plasma via a standard protocol using a refrigerated centrifuge, and plasma was stored at $-80^{\circ} \mathrm{C}$ until the determination of CPT concentrations. Samples were assayed for the quantitation of conjugated and free CPT concentrations using a validated assay involving liquid chromatography (LC) with tandem mass spectrometric (MS/MS) detection, as previously described. ${ }^{1}$ Quantitation of all samples were within the pre-specified calibration ranges (10 to $10,000 \mathrm{ng} / \mathrm{mL}$ and 1 to $1,000 \mathrm{ng} / \mathrm{mL}$ for total and free CPT, respectively), with acceptable quantitation meeting FDA guidance criteria for precision and accuracy of $\leq 15 \%$ relative error. ${ }^{18}$ Nanoparticle-bound CPT concentrations were determined via the subtraction of the free 
CPT quantitation from the total CPT quantitation: conjugated and free CPT were utilized for observations in the development of this model.

A population approach based on non-linear mixed effects modeling using the software package NONMEM version 7.4.3 (Icon, Hanover, MD, USA) was applied. Pirana version 2.9.9, PsN version 4.9, and $\mathrm{R}$ version 3.6.1 were used for workflow management, data handling, and data visualization, respectively. ${ }^{19}$ The first order conditional estimation option with interaction between random and residual error components (FOCE-I), as implemented in NONMEM, was used as the estimation method.

\section{Pharmacokinetic Model-Building Procedure}

Models were generated using custom differential equations via the ADVAN13 subroutine. At the start of structural model development, conjugated CPT disposition was evaluated separately using one-, two-, and three compartment models with firstorder kinetics. After fitting a linear two-compartment model to describe conjugated CPT, free CPT was incorporated into the model via testing of separate one-, two-, and three-compartment models with first-order kinetics (selection based on objective function value improvement and goodness-of-fit plots). Clearance terms to characterize transfer between conjugated and free CPT compartments and clearance of CPT from the model were also evaluated. Since the fraction of conjugated CPT converted to free CPT is unknown, all parameter estimates of free CPT were estimated relative to this fraction. For the model development of free CPT, we assumed that CPT release from the nanoparticle formulation only occurs in the central compartment of conjugated CPT. 
Accumulation of NLG207 nanoparticles in tumor tissue would yield pH-dependent release of CPT, however, within the first 48 hours post-dose, the release rate in (tumor) tissue would be negligible with respect to the rate observed in plasma; at the physiological $\mathrm{pH}$ of 7.4, the CPT release rate is faster in comparison to that of (tumor) tissue, presumed to have $\mathrm{pH}$ values of 5-6.7,20,21

Between-subject variability (BSV) following a log-normal distribution was implemented into the model:

$\theta_{i}=\theta_{\text {pop }} \times e^{\eta_{i}}$ Equation 1

where $\theta_{i}$ represents the individual (post-hoc) value of the parameter for the $i^{\text {th }}$ individual, $\theta_{p o p}$ represents the population mean for the parameter, and $\eta_{i}$ predicts the empirical Bayesian estimate for BSV of $i^{\text {th }}$ individual, sampled from a normal distribution with a mean of 0 and a variance of $\omega^{2}$. Between occasion variability (BOV) was not considered in this model, as only 5 patients had samples for two cycles of treatment.

Residual error was determined separately for both conjugated and free CPT observations, assessed via incorporation of proportional error, additive error, or combination:

$C_{i, j, o b s}=C_{i, j, p r e d} \times\left(1+\varepsilon_{\text {proportional }}\right)+\varepsilon_{\text {additive }}$ Equation 2 where $C_{i, j}$ is the observed or predicted value for subject $i$ at time $j, \varepsilon_{\text {proportional }}$ is the proportional error component, and $\varepsilon_{\text {additive }}$ is the additive error component. Residual error components are sampled from a normal distribution with a mean of zero and a variance of $\sigma$. Combination error models were preferred in the development of this model. 


\section{Covariate Model}

A limited set of potential predictors (covariates) for variability in PK parameters were assessed following structural and stochastic PK model development. Evaluated covariates included patient-related (e.g. actual body weight [BW], age, renal function) and treatment-related (e.g. cycle of treatment) factors. Continuous and binary categorical covariates were investigated using the following equations, respectively:

$\theta_{i}=\theta_{p o p} \times\left(\frac{\operatorname{cov}_{i}}{\operatorname{cov}_{m e d}}\right)^{\theta_{c o v}} \times e^{\eta_{i}}$

Equation 3

$\theta_{i}=\theta_{\text {pop }} \times \theta_{\text {cov }}{ }^{\operatorname{cov}_{i}} \times e^{\eta_{i}}$

\section{Equation 4}

where $\theta_{\text {cov }}$ is the parameter estimate for the specified covariate, $\operatorname{cov}_{i}$ is the covariate value for the $i^{\text {th }}$ individual and $\operatorname{cov}_{\text {med }}$ represents the median, or typical, value for the covariate in the population. The influence of body weight (BW) on PK parameters were either estimated or implemented via standard allometric scaling, with fixed exponents of 1 and 0.75 for volume of distribution and clearance terms, respectively, using Equation

3; a more involved evaluation of allometric scaling beyond traditional implementation was done to 1) determine potential differences in the effect of body weight on conjugated CPT versus free CPT and 2) to determine if clearance terms representative of $\mathrm{pH}$-dependent drug release were influenced by body weight.

\section{Pharmacokinetic Model Evaluation}

Estimates of the structural and covariate model were deemed relevant only if scientifically and biologically plausible. Covariates were plotted against empirical Bayes estimates of BSV to elucidate potential parameter-covariate relationships. Addition of 
one parameter in hierarchical models was evaluated on the basis of objective function value (OFV), which is equal to minus twice the log-likelihood and assumed to follow a Chi-square distribution; a change in OFV $(\triangle \mathrm{OFV})$ of -3.84 corresponded to a $\mathrm{p}$-value of 0.05 (i.e. one degree of freedom). Forward inclusion and backwards elimination of a parameter required a significance level $\mathrm{p}<0.005(-7.9 \Delta \mathrm{OFV})$ and $\mathrm{p}<0.001(-10.8$ $\Delta \mathrm{OFV})$, respectively.

Standard goodness-of-fit (GOF) plots were generated to assess the model's ability to appropriately characterize the data. These plots included observed concentrations plotted against population and individual-predicted concentrations, and conditional weighted residuals (CWRES) versus time and predicted concentrations. To further assess the value of model predictions, visual predictive checks (VPCs) were created to compare distributions of the simulated observations to real data observations. GOF and VPCs for conjugated and free CPT were evaluated independently, with separate plots generated for each set of observations.

Estimations of parameter uncertainty were obtained using the sampling importance resampling (SIR) method, an optimal approach to assess models with small datasets. ${ }^{22}$ Initial proposal density was approximated in absence of reliable \$COVARIANCE output, with relative standard error (RSE) set to 25, 40 and 25 for THETA, OMEGA, and SIGMA parameters, respectively. The SIR method was run for 6 iterations with samples set to 5000 for each iteration and an increasing number of resamples for each iteration until iteration 4 (i.e. 200, 400, 500, 1000, 1000, and 1000). Correlations between parameter estimates were evaluated to ensure identifiability of 
each parameter specified and 95\% confidence intervals (CI) for each parameter was reported in addition to standard RSE estimates, frequently with asymmetric CI. Values below $30 \%$ for shrinkage (BSV and residual error) were deemed acceptable.

\section{RESULTS}

\section{Patients and Samples}

A total of 27 patients with a median age of 60 were included in this study (Table 1). All patients received a $12 \mathrm{mg} / \mathrm{m}^{2}$ dose of NLG207 at the start of cycle one of treatment. Five patients received cycle 6 day 1 treatment, including two patients who had dose reductions ( $50 \%$ and $75 \%$ ). In total, 239 total plasma samples collected over 32 total doses of NLG207 were included in the analysis. Each sample was quantitated for conjugated and free CPT, resulting in 477 total observations, with the omission of one observation due to incorrect sample handling. In samples collected within the first 3 hours post-start of infusion, the observed average percent free CPT was $3.13 \pm 0.21 \%$; incremental increases in average percent free CPT occurred throughout the dosing interval, with a reported value of $20.42 \pm 0.44 \%$ by approximately 50 hours post-dose. None of the observations included were below or above the limit of quantitation for the validated assay.

\section{Structural Model Development}

Disposition of conjugated CPT alone was first evaluated using linear one-, twoand three-compartment models with first order kinetics. The two-compartment and three-compartment models provided significant improvements over the one- 
compartment model, where the three-compartment model provided the lowest OFV. Incorporation of free CPT in the model was initially performed with the twocompartment conjugated CPT model, with comparisons to the three-compartment model to occur later in model development. Body weight (BW) was included as a covariate using standard allometric scaling, which reduced OFV by 70.56 units at this stage.

Next, conversion of CPT from conjugated to free states was addressed.

Conversion of CPT was first modeled using a first order clearance term $\left(C L_{B}\right)$, which resulted in underprediction of unconjugated concentrations at early timepoints after infusion. It was hypothesized that the initial release rate of CPT from the nanoparticles was higher, relating to fast release of CPT molecules from the outer surface of the nanoparticle. Gradually, this release rate will lower and reach steady-state. This was modelled with an initial fast clearance term $\left(C L_{F}\right)$, which showed a first-order decrease over time eventually reaching the slow steady-state clearance $\left(C L_{B}\right)$. The half-life $\left(t_{1 / 2}\right)$ of the fast clearance component was estimated to be 0.307 hours, indicating a very rapid decrease in the release rate of CPT from the nanoparticles over time. Incorporating both terms for $C L_{1}$ provided an increase in fit with a $\triangle \mathrm{OFV}$ of -325 units. The final model is schematically depicted in Figure 1.

Following the fit of parameters associated with CPT release, the threecompartmental model of conjugated CPT no longer provided a better fit of the data, justifying use of the two-compartment conjugated CPT model. The free CPT component of the model was then assessed via linear one-, two-, and three-compartment models. The addition of a peripheral compartment resulted in a better model fit in comparison to 
only a central compartment $(\Delta \mathrm{OFV}=-50.4)$, with no additional benefit of two peripheral compartments.

\section{Stochastic Model Development}

BSV estimates were identifiable for $V_{1}, C L_{B}, C L_{F}, V_{3}$, and $C L_{3}$ with $\eta$-shrinkage below 10\% (Table 2). Furthermore, BSV of $V_{1}$ and $C L_{B}$ and between $V_{3}$ and $C L_{3}$ were highly positively correlated. The proportional plus additive residual error model for both conjugated and free CPT best accounted for unexplained variability of the observed concentrations, with reported $\varepsilon$-shrinkage values of $\leq 10 \%$.

\section{Covariate Model}

Incorporation and refinement of clinically relevant covariates into the model was evaluated following the finalization of the stochastic model, starting with BW. $C L_{F}$ and $t_{1 / 2}$ were omitted from BW allometric scaling, as removal of the terms did not impact OFV and biologic plausibility was minimal; $C L_{F}$ and its associated $t_{1 / 2}$ are parameters influenced more so via physicochemical properties of the nanoparticle formulation in a $\mathrm{pH}$ neutral environment, thus neither were supposed to be influenced by BW. Free CPT allometric scaling was assumed to follow standard fixed allometric scaling terms, with exponents fixed to 1 and 0.75 for volume and clearance terms, respectively. ${ }^{23}$ Alteration to allometric scaling of the conjugated CPT parameters was hypothesized based on proposed differences in nanoparticle size in comparison to small molecules. The exponents of the BW effect on $V_{1}, V_{2}, Q_{1}$, and $C L_{B}$ were estimated, yielding near equivalent values of 1.00 for each term but not providing a more optimal fit, thus 
justifying reversion to standard fixed exponents (i.e. 1.00 for volume of distribution terms, 0.75 for clearance terms), as described in Table 2. Sex and age did not significantly influence parameter estimates. Race and tumor type, though interesting parameters to consider, were not feasible to assess due to the small sample size and the limited dataset. Additional physiologically-based covariates that may influence free CPT PK, including serum albumin level and eGFR, ${ }^{24}$ were also not reasonable to assess due to dataset limitations.

\section{Model Evaluation and Interpretation}

Parameter estimates and respective 95\% confidence intervals (CI) of the final model are summarized in Table 2. All parameters were estimated with good to reasonable precision and verified identifiability of all parameters included in the model. Population and individual predictions were consistent with the observed data for both conjugated and free CPT, as shown via the GOF plots (Figure 2). Conditional weighted residuals were symmetrically distributed in plots stratified by model prediction and time, consistent with minimal bias based on these parameters. VPCs confirmed that the observed data was consistent with simulated observations generated from the model (Figure 3, S1). Inspection of the data is consistent with the formulation retaining a significant portion of total CPT during the first 48 hours post-dose, as shown via the significantly higher exposure of conjugated $\mathrm{CPT}$ in comparison to free $\mathrm{CPT}$ and the delayed maximum concentration $\left(\mathrm{C}_{\max }\right)$ of free $\mathrm{CPT}$. 


\section{DISCUSSION}

To our knowledge, this is the first popPK model describing NLG207 pharmacokinetics beyond non-compartmental analysis (NCA). A critical objective of this model was to combine both conjugated and free CPT pharmacokinetics into a single model to better characterize the NDC formulation mechanistically. The final model utilized a minimal patient population with diverse tumor types to describe conjugated and free CPT disposition, and, importantly, the release of CPT from NDCs.

A rapid spike in free CPT concentrations early post-administration of NLG207, an observation noted in the previously published $\mathrm{NCA},{ }^{14}$ provided rationale to pursue the population approach to better characterize CPT release. By implementing the composite clearance term to characterize $C L_{1}$, we were able to estimate first order release $\left(C L_{B}\right)$ for later timepoints while estimating faster release $\left(C L_{F}\right)$ that dissipates via a first order decay with estimated half-life, $t_{1 / 2}$. Though the $t_{1 / 2}$ estimate would suggest minimal influence of $C L_{F}$ on CPT release by 1.8 hours (or $\sim 6$ half-lives), the relatively large estimate of $C L_{F}$ results in near equal contribution of $C L_{B}$ and $C L_{F}$ at this timepoint; only by 4 hours, or $\sim 13$ half-lives, does the influence of $C L_{F}$ on CPT release become negligible. The calculated half-life associated with CPT release (i.e. $C L_{1}$ ) thus ranges from $0.38 \mathrm{~h}$ immediately at start of infusion to $22 \mathrm{~h}$ at 4 hours post-start of infusion through the remainder of the dosing cycle. Our hypothesis states the outer surfaces of nanoparticles are responsible for the initial rapid CPT release; self-folding properties of the co-polymer formulation leave a small fraction of CPT molecules exposed, enabling hydrolysis from NDCs in the presence of physiological plasma $\mathrm{pH}$ with possible influence of esterase activity. Direct in vitro incubation of the co-polymer lyophilized 
powder in human plasma resulted in a similarly rapid release half-life of 1.7 hours. ${ }^{20}$ Previous data has also suggested that influence of esterase activity provides a minor contribution to overall CPT release, as indicated by a release half-life of 26 hours in prefrozen serum. ${ }^{24}$ a value close to our $C L_{B}$ estimate. It is reasonable to suggest both $\mathrm{pH}$ dependent and esterase-mediated hydrolysis contribute to early release rates, before stabilization of NDCs in plasma enables a first-order process to fully dictate release of CPT.

The linear two-compartment model of conjugated CPT demonstrated significant retention of CPT in the NDC formulation. Volume of distribution estimates in central and peripheral compartments ( $V_{l}$ and $V_{2}$, respectively) were relatively low, consistent with high concentrations in both defined compartments and the physical chemical properties of the drug similar to macromolecules (e.g. monoclonal antibodies). ${ }^{25}$ Similarly, the prior NCA reported a mean steady-state volume of distribution of 4.63 $\pm 1.07 \mathrm{~L}(\mathrm{n}=6)$ and $2.42 \pm 0.7(\mathrm{n}=36)$ for 12 and $15 \mathrm{mg} / \mathrm{m}^{2}$ doses, respectively. ${ }^{14}$ The estimate of inter-compartmental clearance, $Q_{l}$, suggests slow equilibration of the conjugated CPT compartments, further supporting long retention in the systemic circulation.

The peripheral compartment of conjugated CPT, though ideally to represent specifically uptake into tumor, is a composite compartment representative of all tissue uptake, including significant uptake in the kidney, liver, and bladder, as noted in preclinical models. ${ }^{26}$ Generally, models of carrier-mediated agents incorporate uptake into these tissues while also including interactions with the mononuclear phagocyte system (e.g. uptake into peripheral blood mononuclear cells [PBMCs] and the spleen); ${ }^{27}$ 
recent evidence has suggested a limited role of phagocyte-mediated uptake with NLG207. ${ }^{28}$ Further, prior studies have confirmed the limited uptake into adjacent healthy tissues, typically absent of fenestrations large enough for nanoparticle uptake. ${ }^{8}$ A limitation to note is the inadequate ability to address CPT release in the peripheral compartments, as though tumor tissue is generally more acidic, ${ }^{29}$ highly perfused tissues (i.e. liver and kidneys) have near equivalent $\mathrm{pH}$ to plasma. ${ }^{30,31}$ NDCs not only stably retain the majority of CPT, but also confine CPT in the plasma circulation and highly perfused tissues, permitting uptake only into tissues with appropriate size exclusion properties.

In comparison to conjugated CPT, the two-compartment free CPT model generated higher volume of distribution $\left(V_{3}\right.$ and $\left.V_{4}\right)$ and inter-compartmental clearance $\left(Q_{3}\right)$ estimates, as expected for a small molecule. Mean central and peripheral compartment volumes of distribution for the free CPT model were 6.7- and 9.3-fold higher, respectively, in comparison to the corresponding estimates for conjugated CPT. Pharmacokinetic parameters were not routinely obtained via NCA during the time of initial CPT clinical trials, however, reported plasma concentration-time curves from 4 patients receiving large doses of free CPT suggests an approximate volume of distribution of 5-6 L. ${ }^{32}$ Estimates of free CPT inter-compartmental clearance describe fast equilibration (half-life of $\sim 16$ minutes) of the central and peripheral compartments. Clearance of free CPT from the central compartment was slow by comparison, with a terminal half-life of 32.4 hours. The aforementioned early CPT trial suggested a similar relationship between clearance terms, as equilibration and terminal half-lives of 4 patients ranged from 18 to 70 minutes and 10.8 to 19.6 hours, respectively. ${ }^{32}$ A two 
compartment model describing topotecan popPK estimated a similar central volume of distribution to free CPT, but higher peripheral volume of distribution and faster clearances. $^{33}$

With respect to free CPT input, we also considered the possibility that a fraction of CPT would be released in the IV bag prior to administration, however, such models were not identifiable. Nonetheless, assuming $100 \%$ of CPT was retained within the nanoparticle formulation prior to infusion was justified based on the reconstitution procedure for NLG207; reconstituted of NLG207 in sterile water for injection (SWFI) is added to dextrose $5 \%$ in water (D5W), which, per manufacturer label, ${ }^{34}$ is noted to have a $\mathrm{pH}$ of 4.3 and thus negligible release of CPT prior to infusion. ${ }^{20}$

Allometrically scaling all parameters (excluding $C L_{F}$ and $t_{1 / 2}$ ) using BW adequately accounted for differences in body size, as the effect of sex did not appreciably contribute to a better model fit. The use of BW, though presenting with a similar fit to the BSA-adjusted model, was preferred given the evidence supporting allometric theory. ${ }^{35}$ Incorporation of BSV revealed significant differences in parameter estimations of $C L_{B}, C L_{F}, C L_{3}$, and, most notably, $V_{3}$. Incorporation of BW only lead to an appreciable decrease in the BSV of $V_{l}$, resulting in $\sim 10 \%$ of the variability being explained by BW. No other significant covariates were identified in the present study.

Limitations of the present model are attributable to the patient population, sample collection, and parameter identifiability. Additional covariates worthy of exploration, including tumor type and compromised renal function, were not feasible given the small patient sample size $(\mathrm{N}=27)$. The mechanistic effect of plasma albumin and LDL on free CPT and nanoparticle unfolding, respectively, also represent potential 
avenues for further model evaluation. ${ }^{24,36}$ Most notably, the model was unable to incorporate an estimate specifically characterizing renally cleared conjugated CPT during the first $24-48 \mathrm{~h}$ post-infusion, accounting for approximately $16 \%$ of the total dose in prior study. ${ }^{14,24}$ Separation of the typical 4-5 strands comprising a single NLG207 nanoparticle reduces particle size, ${ }^{8}$ thereby increasing the likelihood of glomerular filtration and likely contributing to observed conjugated CPT in urine. Accurate characterization of conjugated CPT renal clearance while accounting for nanoparticle unfolding would require incorporation of serial urine sample collection into the model.

The resulting model reinforced critical aspects of the NDC formulation, while providing an enhanced understanding of CPT release. Conjugated CPT PK had a small volume of distribution, low distribution clearance, and low final central clearance, characteristics consistent with the nanoparticle behavior of FDA-approved liposomal formulations (e.g. irinotecan, doxorubicin, daunorubicin). ${ }^{37-39}$ A similar model characterizing liposomal irinotecan popPK utilized a clearance term consistent with saturable elimination (as implemented via the Michaelis-Menten equation) to describe the release of irinotecan from liposomes in plasma. In comparison to NLG207 PK, high initial release of irinotecan from liposomes is not present and free irinotecan exposure is considerably less, as indicated via the free drug central volume of distribution estimate of $401 \mathrm{~L} .{ }^{37}$ Properties unique to NLG207, including co-polymer self-assembly and $\mathrm{pH}$ dependent release, delineate differences in free drug exposure from that of liposomal formulations; the composite clearance term for CPT release further emphasizes these differences, while also aiding mechanistic interpretation. 


\section{CONCLUSION}

NLG207 pharmacokinetics was best described using a harmonized model, combining separate two-compartment models of nanoparticle-bound and free CPT. Transfer between these model components incorporated two clearance terms to characterize rapid CPT release early post-dose and gradual conversion to a stable first order rate of CPT release from the nanoparticle formulation. Allometric scaling based on body weight also accounted for between-subject variability. The current analysis of this small patient population provides a strong foundation for future study as a potential predictive tool with the availability of data from a larger sample size.

\section{Acknowledgements}

We thank the nursing staff of National Cancer Institute and the fellows of the Genitourinary Malignancies Branch at National Cancer Institute for their care of our patients; Peraton for data management assistance. Most importantly, we appreciate the patients with cancer who enroll in investigational trials to advance the knowledge of this disease.

\section{Grant Support}

Our studies are supported by the Center for Cancer Research, the Intramural Program of the US National Cancer Institute. 


\section{Disclaimer}

The content of this publication does not necessarily reflect the views or policies of the

Department of Health and Human Services, nor does mention of trade names,

commercial products, or organization imply endorsement by the U.S. Government. 


\section{REFERENCES}

1. Schmidt KT, Peer CJ, Huitema ADR, et al. Measurement of NLG207 (formerly CRLX101) nanoparticle-bound and released camptothecin in human plasma. J Pharm Biomed Anal 2020;181:113073.

2. Wall ME, Wani MC. Camptothecin and taxol: from discovery to clinic. J Ethnopharmacol 1996;51:239-53; discussion 53-4.

3. James ND, de Bono JS, Spears MR, et al. Abiraterone for Prostate Cancer Not Previously Treated with Hormone Therapy. N Engl J Med 2017;377:338-51.

4. Moertel CG, Schutt AJ, Reitemeier RJ, Hahn RG. Phase II study of camptothecin (NSC-100880) in the treatment of advanced gastrointestinal cancer. Cancer Chemother Rep 1972;56:95-101.

5. Gottlieb JA, Luce JK. Treatment of malignant melanoma with camptothecin (NSC-100880). Cancer Chemother Rep 1972;56:103-5.

6. Thomas A, Pommier Y. Targeting Topoisomerase I in the Era of Precision Medicine. Clin Cancer Res 2019.

7. Davis ME. Design and development of IT-101, a cyclodextrin-containing polymer conjugate of camptothecin. Adv Drug Deliv Rev 2009;61:1189-92.

8. Clark AJ, Wiley DT, Zuckerman JE, et al. CRLX101 nanoparticles localize in human tumors and not in adjacent, nonneoplastic tissue after intravenous dosing. Proc Natl Acad Sci U S A 2016;113:3850-4.

9. Chao J, Lin J, Frankel P, et al. Pilot trial of CRLX101 in patients with advanced, chemotherapy-refractory gastroesophageal cancer. J Gastrointest Oncol 2017;8:962-9. 10. Keefe SM, Hoffman-Censits J, Cohen RB, et al. Efficacy of the nanoparticledrug conjugate CRLX101 in combination with bevacizumab in metastatic renal cell carcinoma: results of an investigator-initiated phase I-IIa clinical trial. Ann Oncol 2016;27:1579-85.

11. Pham E, Birrer MJ, Eliasof S, et al. Translational impact of nanoparticle-drug conjugate CRLX101 with or without bevacizumab in advanced ovarian cancer. Clin Cancer Res 2015;21:808-18.

12. Sanoff HK, Moon DH, Moore DT, et al. Phase I/II trial of nano-camptothecin CRLX101 with capecitabine and radiotherapy as neoadjuvant treatment for locally advanced rectal cancer. Nanomedicine 2019;18:189-95.

13. Voss MH, Hussain A, Vogelzang N, et al. A randomized phase II trial of CRLX101 in combination with bevacizumab versus standard of care in patients with advanced renal cell carcinoma. Ann Oncol 2017;28:2754-60.

14. Weiss GJ, Chao J, Neidhart JD, et al. First-in-human phase $1 / 2$ a trial of CRLX101, a cyclodextrin-containing polymer-camptothecin nanopharmaceutical in patients with advanced solid tumor malignancies. Invest New Drugs 2013;31:986-1000. 15. Rapisarda A, Uranchimeg B, Sordet O, Pommier Y, Shoemaker RH, Melillo G. Topoisomerase I-mediated inhibition of hypoxia-inducible factor 1: mechanism and therapeutic implications. Cancer Res 2004;64:1475-82.

16. Duska L, O'Malley DM, Krasner C, et al. Abstract CT151: A Phase II study of NLG207 (formerly CRLX101) in combination with weekly paclitaxel in patients with 
recurrent or persistent epithelial ovarian, fallopian tube or primary peritoneal cancer. Cancer Res 2019;79:Suppl.

17. Takahashi N, Surolia I, Thomas A. Targeting DNA Repair to Drive Immune Responses: It's Time to Reconsider the Strategy for Clinical Translation. Clin Cancer Res 2020.

18. Administration UFaD. Bioanalytical Method Validation Guidance for Industry. 2018.

19. Keizer RJ, Karlsson MO, Hooker A. Modeling and Simulation Workbench for NONMEM: Tutorial on Pirana, PsN, and Xpose. CPT Pharmacometrics Syst Pharmacol 2013;2:e50.

20. Cheng J, Khin KT, Jensen GS, Liu A, Davis ME. Synthesis of linear, betacyclodextrin-based polymers and their camptothecin conjugates. Bioconjug Chem 2003;14:1007-17.

21. Young C, Schluep T, Hwang J, Eliasof S. CRLX101 (formerly IT-101)-A Novel Nanopharmaceutical of Camptothecin in Clinical Development. Curr Bioact Compd 2011;7:8-14.

22. Dosne AG, Bergstrand M, Harling K, Karlsson MO. Improving the estimation of parameter uncertainty distributions in nonlinear mixed effects models using sampling importance resampling. J Pharmacokinet Pharmacodyn 2016;43:583-96.

23. West GB, Brown JH, Enquist BJ. A general model for the origin of allometric scaling laws in biology. Science 1997;276:122-6.

24. Eliasof S, Lazarus D, Peters CG, et al. Correlating preclinical animal studies and human clinical trials of a multifunctional, polymeric nanoparticle. Proc Natl Acad Sci U S A 2013; 110:15127-32.

25. Dirks NL, Meibohm B. Population pharmacokinetics of therapeutic monoclonal antibodies. Clin Pharmacokinet 2010;49:633-59.

26. Schluep T, Hwang J, Hildebrandt IJ, et al. Pharmacokinetics and tumor dynamics of the nanoparticle IT-101 from PET imaging and tumor histological measurements.

Proc Natl Acad Sci U S A 2009;106:11394-9.

27. Petschauer JS, Madden AJ, Kirschbrown WP, Song G, Zamboni WC. The effects of nanoparticle drug loading on the pharmacokinetics of anticancer agents. Nanomedicine (Lond) 2015;10:447-63.

28. Chen YF, Wang YH, Lei CS, Changou CA, Davis ME, Yen Y. Host immune response to anti-cancer camptothecin conjugated cyclodextrin-based polymers. J Biomed Sci 2019;26:85.

29. Kato Y, Ozawa S, Miyamoto C, et al. Acidic extracellular microenvironment and cancer. Cancer Cell Int 2013;13:89.

30. Park R, Leach WJ, Arieff AI. Determination of liver intracellular $\mathrm{pH}$ in vivo and its homeostasis in acute acidosis and alkalosis. Am J Physiol 1979;236:F240-5.

31. Skelton LA, Boron WF, Zhou Y. Acid-base transport by the renal proximal tubule. J Nephrol 2010;23 Suppl 16:S4-18.

32. Gottlieb JA, Guarino AM, Call JB, Oliverio VT, Block JB. Preliminary pharmacologic and clinical evaluation of camptothecin sodium (NSC-100880). Cancer Chemother Rep 1970;54:461-70. 
33. Mould DR, Holford NH, Schellens JH, et al. Population pharmacokinetic and adverse event analysis of topotecan in patients with solid tumors. Clin Pharmacol Ther 2002;71:334-48.

34. Hospira. 5\% Dextrose Injection, USP: Package Insert. 2005.

35. Anderson BJ, Holford NH. Mechanistic basis of using body size and maturation to predict clearance in humans. Drug Metab Pharmacokinet 2009;24:25-36.

36. Fleury F, Ianoul A, Berjot M, Feofanov A, Alix AJ, Nabiev I. Camptothecinbinding site in human serum albumin and protein transformations induced by drug binding. FEBS Lett 1997;411:215-20.

37. Wu H, Infante JR, Keedy VL, et al. Population pharmacokinetics of PEGylated liposomal CPT-11 (IHL-305) in patients with advanced solid tumors. Eur J Clin Pharmacol 2013;69:2073-81.

38. Amantea MA, Forrest A, Northfelt DW, Mamelok R. Population pharmacokinetics and pharmacodynamics of pegylated-liposomal doxorubicin in patients with AIDS-related Kaposi's sarcoma. Clin Pharmacol Ther 1997;61:301-11. 39. Hempel G, Reinhardt D, Creutzig U, Boos J. Population pharmacokinetics of liposomal daunorubicin in children. Br J Clin Pharmacol 2003;56:370-7. 


\section{TABLES AND FIGURES}

Table 1. Patient Characteristics

\begin{tabular}{|c|c|}
\hline & $\mathrm{N}=27(\%)$ \\
\hline \multicolumn{2}{|l|}{ General Characteristics } \\
\hline Median age in years & $60(47-76)^{\&}$ \\
\hline Median body weight in $\mathrm{kg}$ & $70.4(46.4-105)^{\&}$ \\
\hline \multicolumn{2}{|l|}{ Gender } \\
\hline Female & $15(55.6)$ \\
\hline Male & $12(44.4)$ \\
\hline \multicolumn{2}{|l|}{ Race } \\
\hline African & $4(14.8)$ \\
\hline Asian & $3(11.1)$ \\
\hline Caucasian & $20(74.1)$ \\
\hline \multicolumn{2}{|l|}{ Tumor Type } \\
\hline Non-small cell lung cancer & $4(14.8)$ \\
\hline Small cell cancer & $3(11.1)$ \\
\hline Pancreatic adenocarcinoma & $3(11.1)$ \\
\hline Cholangiocarcinoma & $3(11.1)$ \\
\hline Ovarian/fallopian tube cancer & $3(11.1)$ \\
\hline Prostate cancer (mCRPC) & $3(11.1)$ \\
\hline Cervical cancer & $2(7.4)$ \\
\hline Colorectal cancer & $2(7.4)$ \\
\hline Mesothelioma & $2(7.4)$ \\
\hline Myxofibrosarcoma & $1(3.7)$ \\
\hline Thymic cancer & $1(3.7)$ \\
\hline \multicolumn{2}{|l|}{ Renal Function } \\
\hline Mild renal impairment $\left(\mathrm{eGFR}=60-90 \mathrm{ml} / \mathrm{min} / 1.73 \mathrm{~m}^{2}\right)$ & $13(48.1)$ \\
\hline Normal renal function (eGFR > $90 \mathrm{ml} / \mathrm{min} / 1.73 \mathrm{~m}^{2}$ ) & $14(51.9)$ \\
\hline Presence of proteinuria & $5(18.5)$ \\
\hline \multicolumn{2}{|l|}{ NCT02769962 (NLG207 + olaparib) } \\
\hline Total & 24 (88.9) \\
\hline Cycle 1 PK collection Only & $19(79.9)^{\#}$ \\
\hline Cycle $1+$ Cycle 6 PK collection & $5(20.1)^{\#}$ \\
\hline \multicolumn{2}{|l|}{ NCT03531827 (NLG207 + enzalutamide) } \\
\hline Total & $3(11.1)$ \\
\hline Cycle 1 PK collection Only & $3(100)^{\#}$ \\
\hline
\end{tabular}

eGFR - estimated glomerular filtration rate

${ }^{\&}$ Range of data reported

${ }^{\$}$ All 3 prostate cancer patients were enrolled on NCT03531827

"Percentage of subgroup data within the selected population 
Table 2. Final population pharmacokinetic parameter estimates

\begin{tabular}{|c|c|c|c|c|c|c|c|}
\hline \multicolumn{4}{|c|}{ Conjugated CPT } & \multicolumn{4}{|c|}{ Free CPT } \\
\hline & Estimate $(95 \%$ CI) & \%RSE & Shr. & & Estimate $(95 \% \mathrm{CI})$ & $\%$ RSE & Shr. \\
\hline \multicolumn{8}{|l|}{ Structural Model } \\
\hline $\begin{array}{c}V_{1}=V_{1,70 \mathrm{~kg}} x\left(\frac{B W}{\mathbf{7 0 k g}}\right)^{1} \\
V_{1,70 \mathrm{~kg}}(L)\end{array}$ & \multirow[t]{2}{*}{$3.16(2.91-3.40)$} & \multirow[t]{2}{*}{$4 \%$} & & $\begin{array}{c}V_{3}=V_{3,70 \mathrm{~kg}} x\left(\frac{B W}{70 \mathrm{~kg}}\right)^{1} \\
V_{3,70 \mathrm{~kg}}(L)\end{array}$ & \multirow[t]{2}{*}{$21.1(12.9-30.5)$} & \multirow[t]{2}{*}{$22 \%$} & \\
\hline $\begin{array}{c}V_{2}=V_{2,70 \mathrm{~kg}} x\left(\frac{B W}{70 \mathrm{~kg}}\right)^{1} \\
V_{2,70 \mathrm{~kg}}(L)\end{array}$ & & & & $\begin{array}{c}V_{4}=V_{4,70 \mathrm{~kg}} x\left(\frac{B W}{70 \mathrm{~kg}}\right)^{1} \\
V_{4,70 \mathrm{~kg}}(L)\end{array}$ & & & \\
\hline $\begin{array}{c}Q_{1}=Q_{1,70 \mathrm{~kg}} x\left(\frac{B W}{70 \mathrm{~kg}}\right)^{0.75} \\
Q_{1,70 \mathrm{~kg}}(\mathrm{~L} / \mathrm{h})\end{array}$ & $0.0482(0.0381-0.0617)$ & $5 \%$ & & $\begin{array}{c}Q_{3}=Q_{3,70 \mathrm{~kg}} x\left(\frac{B W}{70 \mathrm{~kg}}\right)^{0.75} \\
Q_{3,70 \mathrm{~kg}}(L / \mathrm{h})\end{array}$ & $25.6(13.6-46.0)$ & $33 \%$ & \\
\hline $\begin{array}{l}C L_{B} \\
=C L_{B, 70 \mathrm{~kg}} x\left(\frac{B W}{70 \mathrm{~kg}}\right)^{0.75} \\
\quad C L_{B, 70 \mathrm{~kg}}(L / h)\end{array}$ & \multirow{2}{*}{$\begin{array}{c}0.0988(0.0870-0.1129) \\
5.71(3.89-7.85) \\
0.307(0.265-0.356)\end{array}$} & \multirow{2}{*}{$\begin{array}{c}7 \% \\
18 \% \\
8 \%\end{array}$} & & $\begin{array}{l}C L_{3} \\
=C L_{3,70 \mathrm{~kg}} \times\left(\frac{B W}{70 \mathrm{~kg}}\right)^{0.75} \\
\quad C L_{3,70 \mathrm{~kg}}(L / \mathrm{h})\end{array}$ & \multirow[t]{2}{*}{$0.874(0.738-1.044)$} & \multirow[t]{2}{*}{$9 \%$} & \\
\hline $\begin{array}{c}C L_{F}(L / h) \\
t_{1 / 2}(h)\end{array}$ & & & & & & & \\
\hline \multicolumn{8}{|l|}{ Random Effects } \\
\hline $\boldsymbol{B S V}_{\boldsymbol{V}_{1}}(\boldsymbol{C V} \%)$ & \multirow{4}{*}{$\begin{array}{c}18.1(12.3-24.7) \\
\quad 0.918^{\#} \\
33.5(25.3-43.7) \\
62.6(44.2-86.4)\end{array}$} & \multirow[t]{2}{*}{$36 \%$} & \multirow[t]{2}{*}{$6 \%$} & $\boldsymbol{B S V}_{V_{3}}(\boldsymbol{C V} \%)$ & \multirow{4}{*}{$\begin{array}{c}79.8(56.8-117) \\
0.884^{\#} \\
42.2(31.8-57.4)\end{array}$} & \multirow[t]{2}{*}{$42 \%$} & \multirow[t]{2}{*}{$5 \%$} \\
\hline$B S V_{V_{1}, C L_{B}}$ (corr.) & & & & $B S V_{V_{3}, C L_{3}}$ (corr.) & & & \\
\hline$B S V_{C L_{B}}(C V \%)$ & & \multirow{2}{*}{$\begin{array}{l}29 \% \\
35 \%\end{array}$} & \multirow{2}{*}{$\begin{array}{l}2 \% \\
5 \%\end{array}$} & $B_{S V} V_{C L_{3}}(C V \%)$ & & \multirow[t]{2}{*}{$33 \%$} & \multirow[t]{2}{*}{$5 \%$} \\
\hline$B S V_{C L_{F}}(C V \%)$ & & & & & & & \\
\hline \multicolumn{8}{|l|}{ Residual Error } \\
\hline Bound $\boldsymbol{R} E_{\text {proportional }}(\%)$ & $12.3(11.2-13.6)$ & $10 \%$ & $9 \%$ & Free $\boldsymbol{R} E_{\text {proportional }}(\%)$ & $24.8(22.3-28.1)$ & $12 \%$ & $10 \%$ \\
\hline Bound $R E_{\text {additive }}(n g / m L)$ & $5.07(1.27-13.86)$ & $64 \%$ & $9 \%$ & Free RE $E_{\text {additive }}(\mathrm{ng} / \mathrm{mL})$ & $0.396(0.061-0.852)$ & $53 \%$ & $10 \%$ \\
\hline
\end{tabular}

$C I$ confidence interval, \%RSE percent relative standard error, $S h r$. shrinkage, $B W$ body weight, $B S V$ between-subject variability, $R E$ residual error, corr. correlation coefficient

95\% CIs for parameter estimates were obtained via the 2.5 and $97.5 \%$ quantile estimates calculated during the SIR analysis.

\# - correlation coefficient for specified covariance term is reported without 95\% CI or \%RSE.

Conjugated CPT parameters: $V_{l}$ central volume of distribution, $V_{2}$ peripheral volume of distribution, $Q_{l}$ intercompartmental clearance $\left(V_{l}-V_{2}\right), C L_{\mathrm{B}}$ base conversion rate of conjugated CPT to free CPT $\left(V_{1}-V_{3}\right), C L_{F}$ time dependent rate conversion of conjugated CPT to free CPT $\left(V_{1}-V_{3}\right), t_{1 / 2}$ half-life of first order decay scalar term governing $C L_{F}$ mediated CPT conversion.

Free CPT parameters: $V_{3}$ central volume of distribution, $V_{4}$ peripheral volume of distribution, $Q_{3}$ intercompartmental clearance $\left(V_{3}-V_{4}\right), C L_{3}$ clearance of free CPT from $V_{3}$

Population estimates $\mathrm{V}_{1,70 \mathrm{~kg}}, \mathrm{~V}_{2,70 \mathrm{~kg}}, \mathrm{Q}_{1,70 \mathrm{~kg}}, \mathrm{CL}_{\mathrm{B}, 70 \mathrm{~kg}}, \mathrm{~V}_{3,70 \mathrm{~kg}}, \mathrm{~V}_{4,70 \mathrm{~kg}}, \mathrm{Q}_{3,70 \mathrm{~kg}}$, and $\mathrm{CL}_{3,70 \mathrm{~kg}}$ correspond to a $70 \mathrm{~kg}$ subject and are adjusted to individual values using the corresponding parameter formulas. 
Figure 1

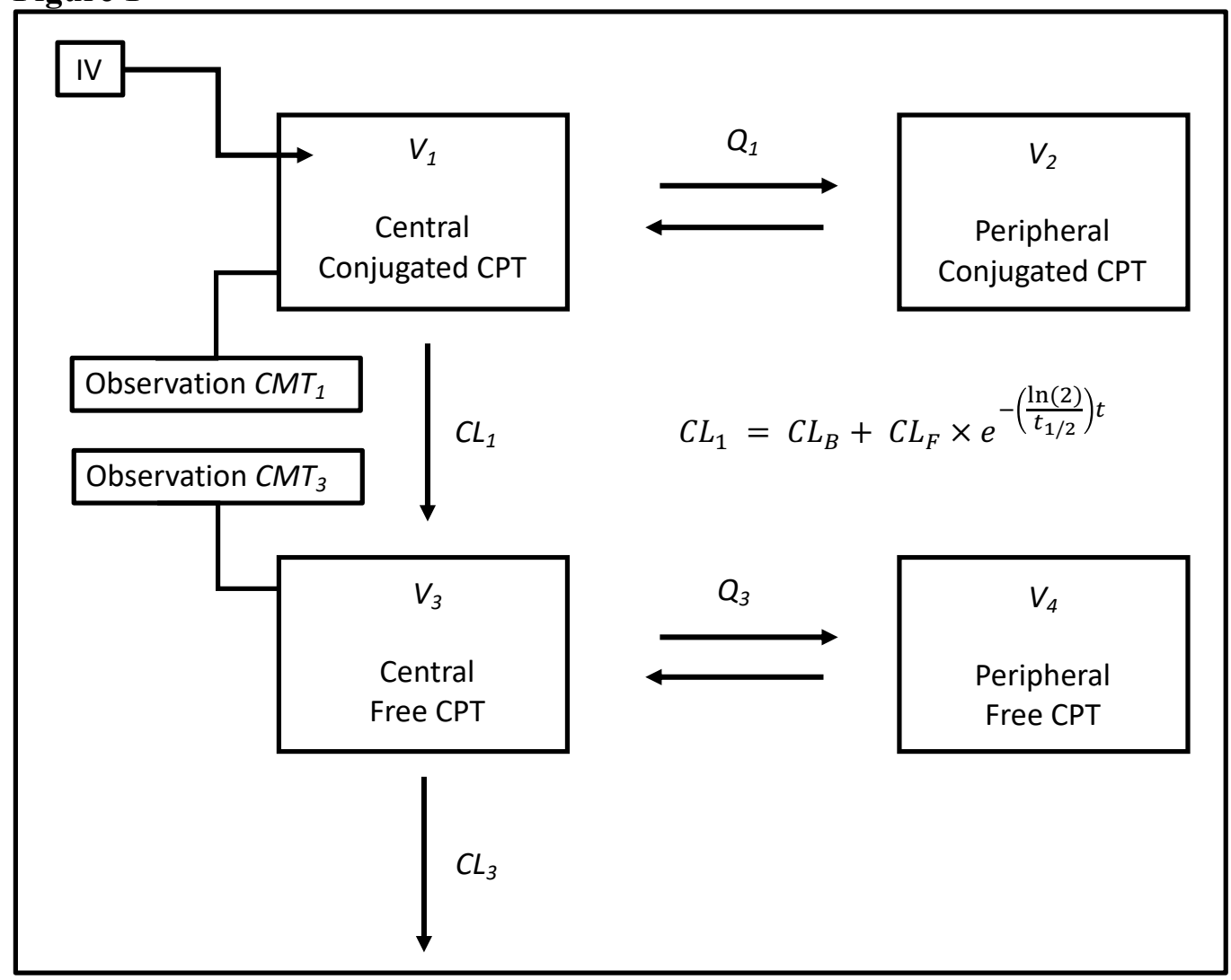

Overview of the four-compartment model describing disposition of nanoparticle-bound (conjugated) and free CPT, and the associated calculated parameters. Plasma sample quantitation enabled $C M T_{1}$ and $C M T_{3}$ observations for conjugated and free CPT concentrations, respectively. The model can be divided into two portions: Conjugated CPT distribution described via estimates $V_{1}, V_{2}$, and $Q_{1}$, and Free CPT distribution described via estimates $V_{3}, V_{4}$, and $Q_{3}$.

$C L_{1}$, or the conversion of conjugated to free $\mathrm{CPT}$, was described using two additive clearance terms: $C L_{B}$ is the "base" rate of conversion, and $C L_{F}$ is a "fast" clearance rate that is modified via a simulated first order decay term with estimated half-life, $t_{1 / 2}$. 
Figure 2

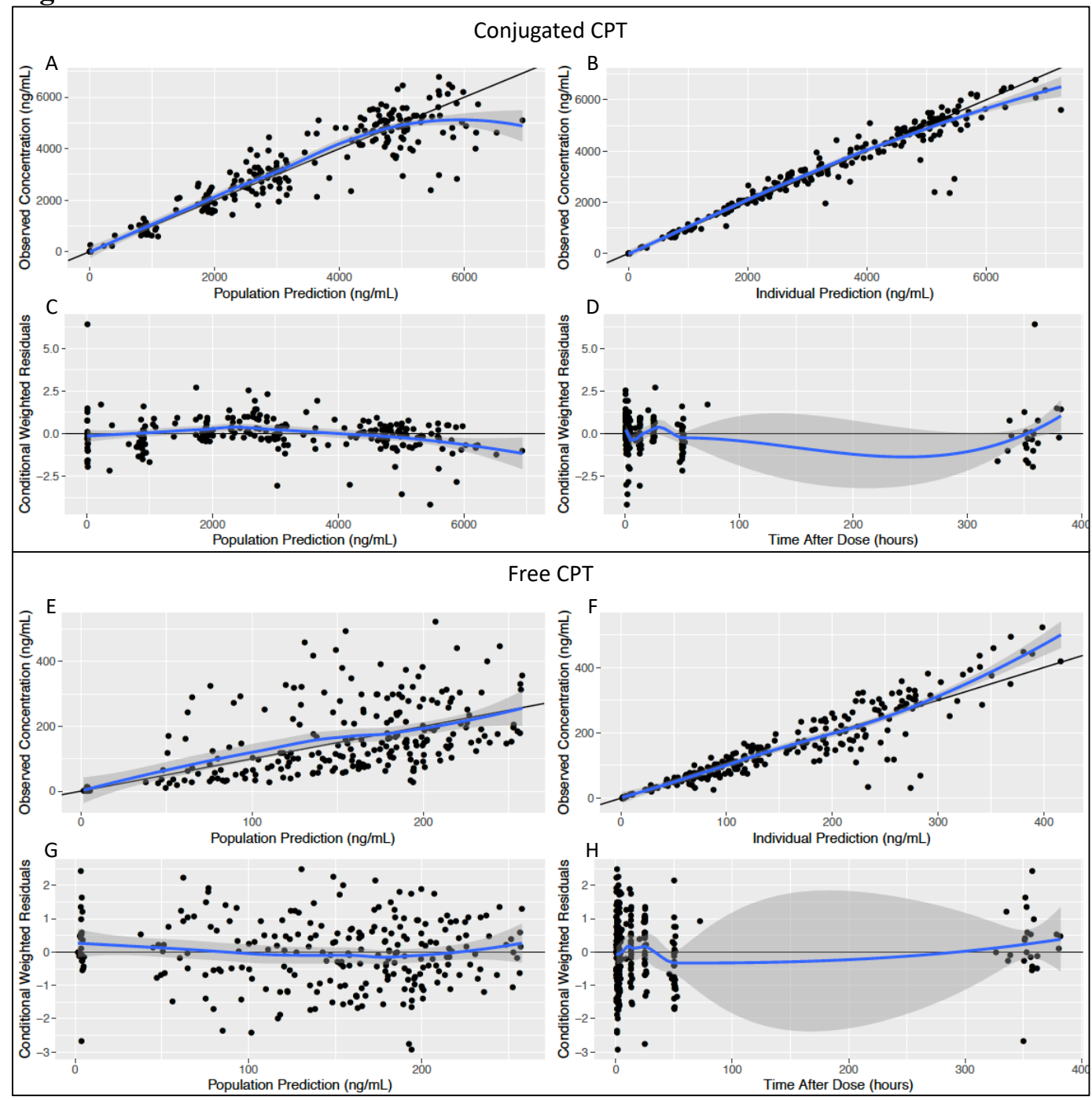

Goodness-of-fit plots for the pharmacokinetic model. Separate plots were generated for the evaluation of conjugated CPT (A-D) and free CPT (E-H). Plots include population prediction against observed data $(\mathbf{A} \& \mathbf{E})$, individual prediction against observed data $(\mathbf{B} \& \mathbf{F})$, conditional weighted residuals against population prediction $(\mathbf{C \& G})$, and conditional weighted residuals against time after dose $(\mathbf{D} \& \mathbf{H})$. The solid black lines represent either the line of unity $(\mathbf{A}, \mathbf{B}, \mathbf{E}$, $\mathbf{F})$ or the zero line $(\mathbf{C}, \mathbf{D}, \mathbf{G}, \mathbf{H})$. Solid blue lines represent the local regression fit of the values. 


\section{Figure 3}
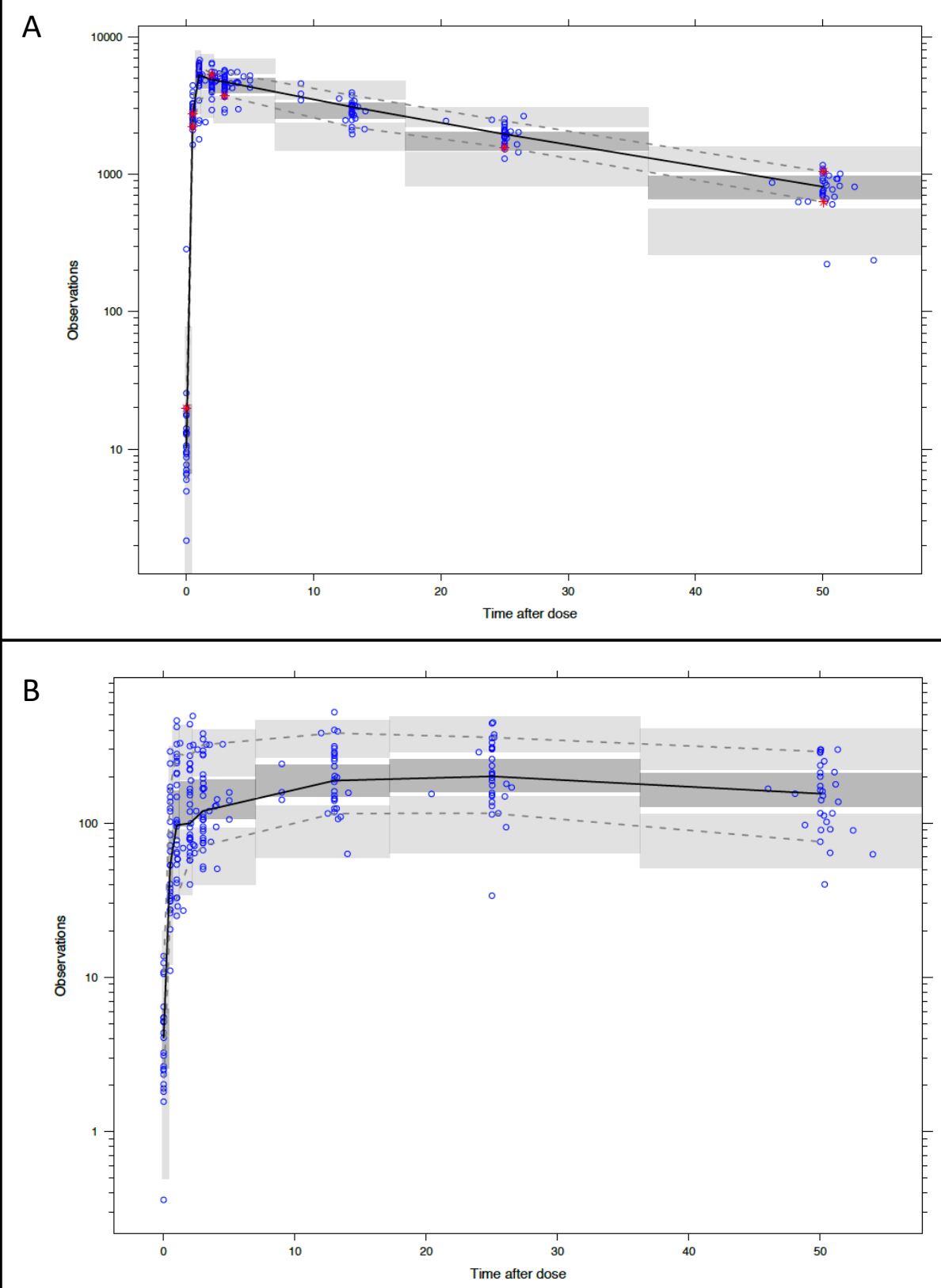

Visual Predictive Checks (VPCs) of Conjugated (A) and Free CPT (B) for timepoints up to $\sim 50$ hours post dose of NLG207. "Observations" are reported in units of ng/mL using log scale axis, and "time after dose" is reported in hours. Solid black lines depict the observed median and dashed lines represent the $2.5 \%$ and $97.5 \%$ percentile concentrations. $95 \%$ prediction intervals of the simulated mean and the 2.5 and $97.5 \%$ percentiles are represented by dark- and light-grey areas, respectively. Round dots represent observations and asterisks highlight observed percentiles outside of the prediction area. 


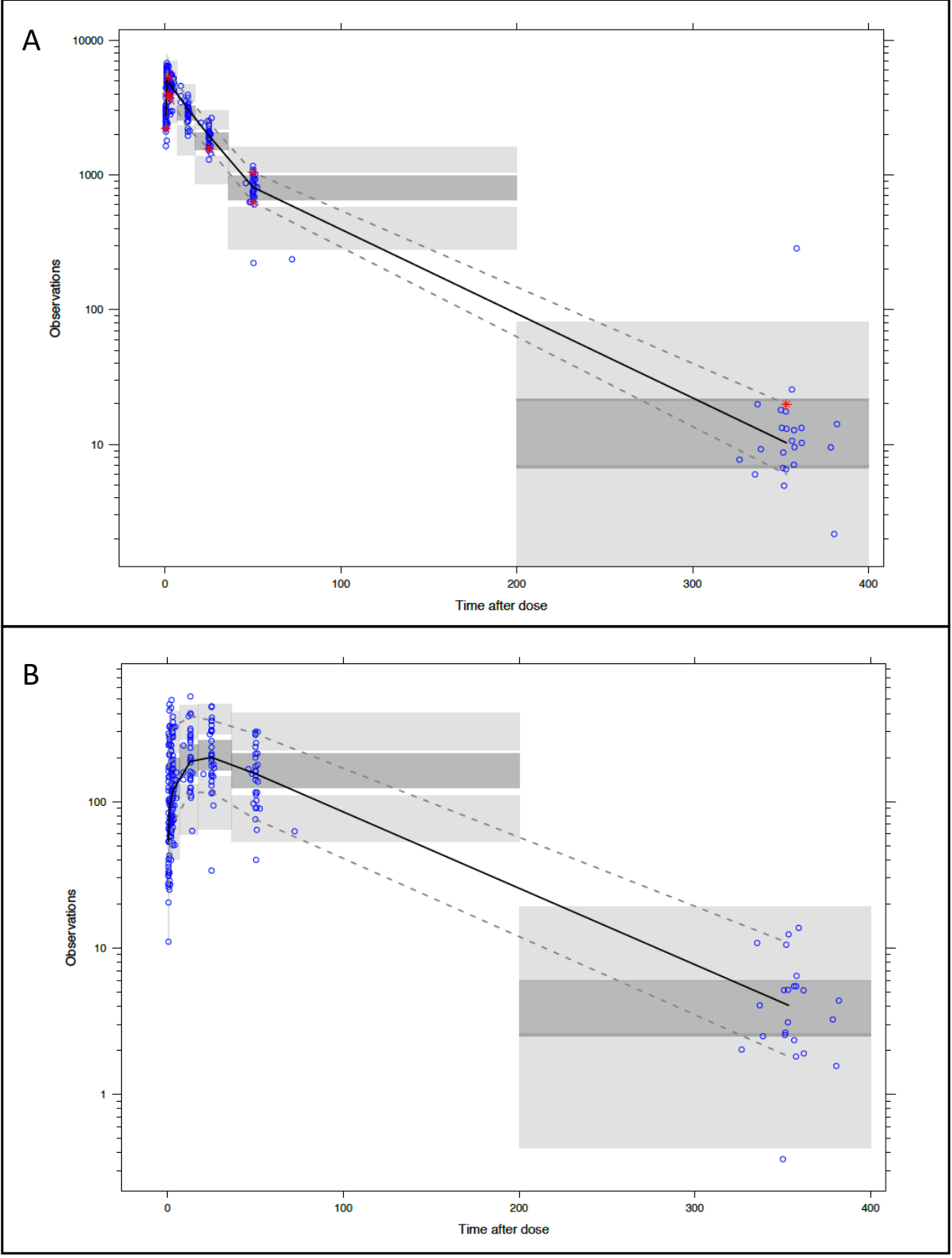

Visual Predictive Checks (VPCs) of Conjugated (A) and Free CPT (B) for timepoints up to $\sim 360$ hours post dose of NLG207. "Observations" are reported in units of ng/mL using log scale axis, and "time after dose" is reported in hours. Solid black lines depict the observed median and dashed lines represent the $2.5 \%$ and $97.5 \%$ percentile concentrations. $95 \%$ prediction intervals of the simulated mean and the 2.5 and $97.5 \%$ percentiles are represented by dark- and light-grey areas, respectively. Round dots represent observations and asterisks highlight observed percentiles outside of the prediction area. 


\section{Chapter 7}

\section{NLG207 (formerly CRLX101) in Combination with Enzalutamide in Patients with Metastatic Castration-Resistant Prostate Cancer Previously Treated with Enzalutamide}

Keith T. Schmidt ${ }^{1}$, Ravi A. Madan², Fatima Karzai², Marijo Bilusic ${ }^{2}$, Lisa M. Cordes ${ }^{2}$, Cindy H. Chau ${ }^{2}$, Cody J. Peer ${ }^{1}$, Susan Wroblewski ${ }^{2}$, Alwin D. R. Huitema ${ }^{3,4}$, James L. Gulley $^{2}$, William L. Dahut ${ }^{2}$, and William D. Figg ${ }^{1,2}$

${ }^{1}$ Clinical Pharmacology Program, Center for Cancer Research, National Cancer Institute, National Institutes of Health, Bethesda, MD, USA

${ }^{2}$ Genitourinary Malignancies Branch, Center for Cancer Research, National Cancer Institute, National Institutes of Health, Bethesda, MD, USA

${ }^{3}$ Dept. Pharmacy \& Pharmacology, Netherlands Cancer Institute, Amsterdam, The Netherlands

${ }^{4}$ Department of Clinical Pharmacy, University Medical Center Utrecht, Utrecht University, Utrecht, The Netherlands

\section{Interim Analysis}




\section{Abstract}

Background: The effectiveness of enzalutamide, a standard of care treatment for patients with metastatic castration-resistant prostate cancer (mCRPC), is limited by numerous mechanisms of acquired resistance, including alterations to the androgen receptor (AR), PI3K/AKT/mTOR, and Wnt/ $/$-catenin pathways. Dual inhibition of Hypoxia-Inducible Factor (HIF)-1 $\alpha$ and the AR have been shown to synergistically down-regulate AR- and HIF-1 $\alpha$-mediated transcription and cell proliferation. NLG207 (formerly CRLX101), a nanoparticle drug-conjugate (NDC) of campothecin shown clinically to be an inhibitor of HIF-1 $\alpha$ accumulation, had significant anti-tumor activity in combination with enzalutamide in preclinical models of enzalutamide resistance. This open-label phase 2 clinical study with a lead-in dose escalation will evaluate the tolerability and efficacy of NLG207 in combination with enzalutamide in patients with mCRPC previously treated with enzalutamide.

Patients and Methods: Following confirmation of the recommended phase 2 dose (RP2D) of NLG207 in combination with enzalutamide via a 3-6 patient lead-in dose escalation, efficacy in patients with mCRPC who previously received enzalutamide will be evaluated using a single arm, optimal two-stage design. Patients will receive NLG207 via IV infusion every 2 weeks and enzalutamide $160 \mathrm{mg}$ orally once daily. The primary endpoint of this study is overall response defined as a PSA decline of at least $50 \%$ or stable disease on imaging following 5 months of treatment. 
Interim Results: As of May 20, 2020, three patients have been accrued to the lead-in dose escalation, with one patient evaluable for dose-limiting toxicity (DLT) and receiving more than one cycle of treatment. Following a $12 \mathrm{mg} / \mathrm{m}^{2}$ dose of NLG207, the evaluable patient experienced Grade 2 non-infective cystitis and hematuria without resolution following a two-week dose delay of NLG207, resulting in a DLT. Though the data is currently limited, PSA declines were observed in 2 of 3 patients, with one patient having a $>50 \%$ PSA decline from baseline after a single dose of NLG207.

Conclusions: Following the first DLT, the lead-in dose escalation will enroll a total of 6 patients to determine a RP2D. Continued enrollment is required to evaluate the clinical anti-tumor activity of NLG207 in combination with enzalutamide.

ClinicalTrials.gov identifier: NCT03531827 


\section{Introduction}

Enzalutamide is a potent second-generation androgen receptor antagonist (SGARA) that has been shown to improve overall survival in patients with metastatic castration-resistant prostate cancer (mCRPC). ${ }^{1,2}$ Additional studies resulted in expanded FDA approval of enzalutamide in other disease states, including for patients with nonmetastatic CRPC (nmCRPC) $)^{3}$ and metastatic castration sensitive prostate cancer (mCSPC). ${ }^{4,5}$ The benefit of SG-ARAs in advanced prostate cancer has led to further development of the drug class and the recent introduction and success of apalutamide and darolutamide. ${ }^{6,7}$ Despite anti-tumor efficacy and improved clinical outcomes, disease progression on SG-ARA therapy is inevitable and treatment duration is limited secondary to acquired resistance. Resistance to enzalutamide, the most frequent of the SG-ARAs to be characterized in the literature, can be manifested via several mechanisms, including alterations of the androgen receptor (AR) and hypoxia-mediated (e.g. PI3K/Akt/mTOR, Wnt/ $\beta$-catenin) signaling pathways (Chapter 2). ${ }^{8,9} \mathrm{AR}$ amplification resulting from AR copy number gain has been frequently noted following enzalutamide treatment and associated with shorter time to progression (TTP). ${ }^{10}$ The AR F877L point mutation has also been noted to switch enzalutamide into an AR agonist in a nonclinical study, ${ }^{11}$ though few cases have been documented clinically. ${ }^{12,13}$ Splice variation of the AR, clinically characterized via expression of the constitutively active AR-V7 variant, has also been correlated with shorter TTP following enzalutamide treatment and investigated as potential biomarker for therapeutic optimization. ${ }^{14-16}$

In addition to AR alterations, intra-tumoral hypoxia has been implicated in facilitating aggressive tumor phenotypes, metastatic potential, and acquired resistance to 
AR-targeted treatments. ${ }^{17-21}$ Notably, hypoxia-inducible factor (HIF)-1 $\alpha$, an upregulated transcription factor in hypoxia, can alter cellular metabolism, promoting tumor angiogenesis, anaerobic metabolism, immunity, adaptation, and invasion. ${ }^{21,22} \mathrm{~A}$ ternary complex comprising AR, HIF- $1 \alpha$, and $\beta$-catenin was initially proposed to facilitate cross-talk of these pathways and increase activation of AR target genes. ${ }^{17,23-25}$ Our laboratory initially interrogated targeting HIF-AR crosstalk via combination HIF-1 $\boldsymbol{\alpha}$ inhibition (achieved via RNA silencing or chetomin treatment) and enzalutamide treatment in vitro; combination treatment synergistically reduced AR- and HIF-1 $\boldsymbol{\alpha}$ regulated transcription, and VEGF protein expression. ${ }^{17}$ Further, the combination treatment resulted in significant inhibition $\mathrm{LNCaP}$ and 22Rv1 cell growth, the latter of which indicated anti-tumor activity against cells with significant AR-V7 expression. ${ }^{17}$ NLG207 (formerly CRLX101), a nanoparticle-drug conjugate (NDC) of the potent topoisomerase I inhibitor, camptothecin, ${ }^{26}$ was previously shown to inhibit HIF$1 \alpha$ protein accumulation and tumor angiogenesis in numerous preclinical models. ${ }^{27-30} \mathrm{~A}$ derivative of camptothecin, topotecan, was previously shown to knockdown HIF-1 $\boldsymbol{\alpha}$, decrease nuclear translocation of full length AR (AR-FL) and AR-V7, and increase sensitivity to enzalutamide. ${ }^{31} \mathrm{HIF}-\mathrm{AR}$ crosstalk was further studied in vivo via treatment with NLG207 and enzalutamide in the 22Rv1 and castrated VCaP xenograft models, which replicate the relevant enzalutamide resistance mechanisms of AR splice variation and AR amplification, respectively. Combination treatment resulted in significant reductions in tumor growth, with an re-sensitization of tumors to enzalutamide demonstrated in castrated mice with implanted $\mathrm{VCaP}$ xenografts. In vitro correlative analyses in VCaP cells demonstrated the capability of NLG207 to down-regulate full- 
length AR and AR-V7, while down-regulating AR target-genes (PSA and ERG) when in combination with enzalutamide (Chapter 4). Based on these findings, we hypothesized that the treatment combination of NLG207 and enzalutamide can overcome mechanisms of enzalutamide resistance and result in clinically meaningful increases in TTP in mCRPC despite prior disease progression on enzalutamide monotherapy.

Clinically, NLG207 has been previously evaluated both as monotherapy and in combination treatments with over 300 patients of multiple tumor histologies receiving at least one dose on clinical trials..$^{29,32-36}$ The nanoparticle formulation, designed to overcome poor physicochemical properties of CPT via conjugation to a self-assembling $\beta$-cyclodextrin-polyethylene glycol (PEG) co-polymer backbone, demonstrates a favorable pharmacokinetic profile by prolonging plasma retention time and controlling rate of drug release. ${ }^{36-38}$ Clinical pharmacokinetic analyses have demonstrated the majority of CPT to be confined within the formulation in plasma at 48 hours postadministration, which in turn limits exposure of free CPT, controls the rate of clearance, and reduces the potential for significant toxicities (myelosuppression, cystitis) (Chapter 6) ${ }^{36}$ Additionally, the formulation has been shown to reduce interaction with mononuclear phagocyte system (MPS) to greatly reduce clearance, ${ }^{39}$ and utilize the enhanced permeation and retention (EPR) effect to facilitate preferential delivery of drug to tumor tissue. ${ }^{38}$ Further, notable decreases in the protein expression of VEGF and CAIX, downstream targets of HIF-1 $\boldsymbol{\alpha}$, via immunohistochemical staining were observed in patients with advanced solid tumors following treatment with NLG207. ${ }^{40}$ The summation of previous clinical evidence supports the clinical investigation of NLG207 to target HIF-1 $\boldsymbol{\alpha}$ in patients with mCRPC, following confirmation of a 
recommended phase II dose (RP2D) of NLG207 in combination with enzalutamide via an abbreviated lead-in dosing phase.

This phase II study (NCT03531827) will evaluate the anti-tumor activity of NLG207 at the RP2D in combination with enzalutamide in patients with advanced mCRPC who previously received enzalutamide monotherapy. Here, an interim analysis of the study is presented.

\section{Patients and Methods}

\section{Study Population}

This study is enrolling patients 18 years or older with progressive mCRPC, defined as either PSA progression (at least two rising PSA levels separated by $\geq$ one week), or radiographic progression (either two or more new lesions on bone scan), or new or growing lesions on computed tomography (CT) imaging per RECISTv1.1. Patients must have previously been treated with enzalutamide for two or more cycles and must have had evidence of disease progression while on treatment. Additionally, patients are required to have an Eastern Cooperative Oncology Group (ECOG) performance status $\leq 2$ with adequate organ and bone marrow function and maintain castrate levels of testosterone $(<50 \mathrm{ng} / \mathrm{dL})$ via orchiectomy or continuous luteinizing hormone releasing hormone agonist/antagonist therapy. With the exception of enzalutamide, the study requires a minimum 14- or 28-day washout for immunotherapy or other treatments (e.g. chemotherapy, hormonal treatment, investigational treatment), respectively; patients are permitted to immediately enroll on-study if actively progressing on enzalutamide during screening. 
The study excludes patients previously treated with anti-hormonal targeted therapy with proposed activity against AR-V7 (e.g. EPI-506, AZD5312); otherwise, patients are allowed to enroll irrespective of current line of therapy or previously utilized agents, so long as documented disease progression on enzalutamide is present. Exclusion criteria include patients with known brain metastases, prior history of seizures, palliative radiation within the past 2 weeks, history of prior invasive malignancy within the past 3 years, microscopic hematuria within the past 7 days or uncontrolled intercurrent illness. Additionally, HIV-positive patients on anti-retroviral therapy may enroll as long as they have been on treatment for at least 5 years with undetectable viral load and CD4 count $>150$ cells/uL, primarily to ensure the avoidance of potential drug-drug interactions with study treatment.

\section{Study Design and Treatment}

This is a single arm, open-label phase II optimal two-stage study with a 3 to 6 patient lead-in dose escalation (Figure 1). At study initiation, the objective is to validate the RP2D of NLG207 when given in combination with enzalutamide, via the lead-in intra-patient dose escalation. An initial cohort of 3 patients will receive NLG207 at 12 $\mathrm{mg} / \mathrm{m}^{2}$ via intravenous infusion over one hour every two weeks, resulting in 2 doses per 28-day cycle. Enzalutamide $160 \mathrm{mg}$ orally once daily will be administered starting on Cycle 1 Day 2 (C1D2) and continue throughout the remainder of the study (Figure 2). After 2 cycles of NLG207 $12 \mathrm{mg} / \mathrm{m}^{2}$ administered in combination with enzalutamide, the NLG207 dose may be escalated to $15 \mathrm{mg} / \mathrm{m}^{2}$ for the third and subsequent cycles of treatment. Dose-limiting toxicities (DLTs) associated with NLG207 treatment will be evaluated from the start of treatment through the end of cycle 3; DLTs include persistent 
Grade 4 hematologic toxicity (neutropenia, anemia, thrombocytopenia), Grade 3 neutropenia complicated by fever, any Grade 3-4 non-hematologic toxicity, or any toxicity resulting in greater than 2 weeks of dose delay or resulting in less than $75 \%$ of total NLG207 dose during cycle 1. G-CSF support is not permitted to facilitate recovery of neutrophil count during the DLT assessment period. If one of 3 patients experience a DLT, the lead-in will be expanded to 6 patients in total. The lead-in dosing schema may permit the evaluation of either NLG207 12 or $9 \mathrm{mg} / \mathrm{m}^{2}$ every two weeks via IV infusion as the R2PD if toxicity is deemed intolerable with the $15 \mathrm{mg} / \mathrm{m}^{2}$ dose. Validation of the RP2D is one of two secondary objectives of this study.

Following confirmation of the RP2D, patients will be enrolled onto the phase II portion of the study. NLG207 will be dosed at the RP2D in combination with enzalutamide similarly to the lead-in phase. The primary objective of the study is to evaluate the anti-tumor activity of combination treatment with respect to treatment response, defined as either $\geq 50 \%$ decline in PSA or stable disease after 5 months on treatment. The Simon optimal two-stage design was used with an alpha $=0.10$ and a beta $=0.20$ to rule out an unacceptable low response rate of $25 \%\left(\mathrm{P}_{0}=0.25\right)$ in favor of an improved response rate of $50 \%\left(\mathrm{P}_{1}=0.50\right)$; the null hypothesis is based on results on the reported rate of $\geq 50 \%$ decline in patients with $\mathrm{mCRPC}$ receiving third line of treatment. ${ }^{41}$ The first stage of accrual will enroll 8 patients (not including those in the lead-in dose escalation), targeting a response in 3 or more patients; if the target response were achieved in the first 8 patients, the cohort would expand to a total of 21 total patients, with responses in 8 or more patients warranting further study. The additional secondary objectives of the study are to evaluate the duration of responses as defined by 
a sustained $>30 \%$ decline in PSA, overall survival, and changes in measurable disease as determined by Response Evaluation Criteria in Solid Tumors (RECIST) v1.1 and Prostate Cancer Working Group 3 (PCWG3). ${ }^{42,43}$

\section{Toxicity Management and Evaluation}

Adverse events (AEs) with respect to NLG207 will be classified and graded according to the National Cancer Institute Common Toxicity Criteria for Adverse Events, version 4.0. For hematologic toxicity, dose delays will be implemented for patients experiencing Grade $\geq 2$ neutropenia, Grade $\geq 3$ anemia, and platelet counts $<100,000$ cells, with the possibility of dose reduction and/or supportive care with G-CSF administration (only in efficacy evaluation) or RBC transfusion depending on severity and length of the AE. Grade $\geq 3$ non-hematologic toxicities require a dose delay for up to 28 days until resolution to Grade 1 or less. Persistent AEs or toxicities requiring $>2$ dose reductions will result in permanent NLG207 discontinuation. In addition to an antiemetic, NLG207 is administered with a corticosteroid, an antihistamine, and an H2 antagonist to reduce the likelihood of hypersensitivity reactions. To reduce the likelihood of non-infective cystitis, a liter of normal saline is infused over 2 hours prior to the NLG207 infusion, followed by an additional liter of normal saline after infusion.

\section{Correlative Analyses}

Several correlative analyses will evaluate the pharmacokinetics of NLG207 and pharmacodynamic biomarkers of combination treatment. To assess NLG207 plasma pharmacokinetics, serial blood collection will occur on cycle 1 day 1 (C1D1) and C2D15 at pre-dose, mid-infusion, end of infusion (EOI), 1, 2, 3, 4, 8, 12, and 24 hours 
post-EOI; the analysis will evaluate NLG207 administered as monotherapy on C1D1 and in combination once enzalutamide is at steady state (C2D15) to determine any potential drug-drug interaction associated with combination therapy. Samples will be collected in $6 \mathrm{~mL}$ sodium heparin tubes (green top, BD Sciences), processed into plasma via a standard protocol using a refrigerated centrifuge, and stored at $-80^{\circ} \mathrm{C}$. Samples will be assayed for the quantitation of bound and free CPT using previously the described validated assay, implementing liquid chromatography (LC) and tandem mass spectrometric detection (MS/MS) (Chapter 5). ${ }^{26}$ Patients will also be randomized to receive $1300 \mathrm{mg}$ sodium bicarbonate by mouth four times daily starting 48 hours prior to infusion through 24 hours post-EOI on either C1D1 or C2D15 to sufficiently alkalinize urine $\mathrm{e}^{44}$ and evaluate renally cleared camptothecin. Over the course of the 24 hour serial plasma collection on C1D1 and C2D15, urine will be collected with each void with time points unspecified. Urine samples will be flash frozen and assayed using $\mathrm{LC}$ and fluorometric detection to determine the ratio of active to inactive (i.e. lactone to carboxylate) camptothecin in each urine sample (assay methods are based on a previously established assay for topotecan). ${ }^{45}$

Pharmacodynamic biomarkers will evaluate numerous aspects of anti-tumor activity following combination treatment, including effects on AR activity, angiogenic signaling and DNA damage. Circulating tumor cells (CTCs), to be collected in Streck ${ }^{\circledR}$ tubes on C1D1, C2D15, C6D1, and at progression, will be enumerated and assessed for alterations in AR expression, genomic instability, and $\boldsymbol{\gamma}-\mathrm{H} 2 \mathrm{AX}$ induction (if feasible) using EPIC Sciences platform. ${ }^{46,47}$ To assess AR-FL and AR-V7 mRNA expression in circulating free DNA (cfDNA), blood will be collected using PAXgene ${ }^{\circledR}$ RNA tubes 
and processed using the PAXgene Blood RNA kit; purified RNA will be reverse transcribed into cDNA, amplified using custom primers and evaluated for fold-change differences in mRNA expression via semi-quantitative real-time polymerase chain reaction (qPCR), as described previously (Chapter 4). At the same timepoints as CTCs, circulating plasma VEGF protein concentrations from samples collected in EDTA tubes (purple top, BD Biosciences) will be evaluated using previously established electrochemiluminescence techniques. ${ }^{48}$ DNA damage response transcriptional signatures from additional whole blood in PAXgene RNA tubes, collected on C1D1, C2D15 and at disease progression and processed as above, will be evaluated via the 192 gene DNA Damage and Repair panel using the NanoString nCounter platform. Lastly, peripheral blood mononuclear cells (PBMCs) will be collected (CPT Citrate tubes, BD Biosciences) on C1D1 and C2D15 to evaluate the differences PAR levels via the previously described "radiation-enhanced-PAR" (REP assay). ${ }^{49}$

\section{Results}

As of May 20, 2020, the study has accrued in total 3 patients, with only one of the three patients eligible for DLT evaluation. Key characteristics for each patient are summarized in Table 1. Patient \#1 received 3 doses of NLG207 before experiencing a DLT after a $12 \mathrm{mg} / \mathrm{m}^{2}$ dose of NLG207, and discontinued NLG207 treatment following further unresolved AEs. Grade 2 non-infective cystitis and hematuria possibly related to NLG207 were reported several days prior to the scheduled C2D15 dose of NLG207, resulting in holding of the NLG207 dose, administration of sodium bicarbonate, and hydration until resolution. With respect to the $\mathrm{C} 2 \mathrm{D} 1$ dose, trough plasma PK samples 
were drawn on C2D13 and C2D15, showing low but detectable levels of both

nanoparticle-bound CPT (19.11 and $13.05 \mathrm{ng} / \mathrm{mL}$, respectively) and free CPT (16.93 and $13.63 \mathrm{ng} / \mathrm{mL}$, respectively). Following a dose delay of two weeks, the AEs did not improve to allow further treatment, leading to a DLT. Both AEs resolved prior to the scheduled dose on C3D15, however, a Grade 2 neutrophil count decrease did not permit the administration of NLG207, leading to permanent discontinuation of NLG207. Of note, grade 2 hematologic AEs (anemia, WBC decrease, lymphocyte decrease) possibly related to NLG207 occurred intermittently during the first cycle of treatment. Grade 2 nausea was reported following the first infusion of NLG207 and deemed possibly related to study treatment. The patient received treatment on-study for 41 weeks until radiographic disease progression was noted, a TTP that is unexpectedly long for a patient with $\mathrm{mCRPC}$ who received 4 previous lines of therapy; however, this cannot be definitively tied to combination drug exposure.

The remaining two patients opted to discontinue treatment prior to the start of cycle 2. Patient \#2 received two $12 \mathrm{mg} / \mathrm{m}^{2}$ doses of NLG207 before requesting removal from study due to a rising PSA, increased alkaline phosphatase level, and self-described right-sided pelvic pain, opting to receive Radium-223 instead; no protocol-related AEs were reported for Patient \#2. Patient \#3 experienced a grade 3 syncopal episode during the C1D15 infusion that was deemed unrelated to NLG207, but possibly related to history of hypertension requiring blood pressure medications (amlodipine, lisinopril, metoprolol) or pre-medication with diphenhydramine; the syncopal episode prompted discontinuation of the C1D15 dose of NLG207 mid-infusion. Grade 3 anemia and grade 2 lymphocyte reduction were also reported in Patient \#3, deemed possibly related to 
NLG207, pre-dose hydration, and potentially a history of upper gastrointestinal bleed and diverticulosis in the case of anemia. Patient \#3 decided to receive standard of care treatment, withdrawing consent prior to the scheduled $3^{\text {rd }}$ dose of NLG207.

Despite limited time on NLG207 therapy, 2 of the 3 patients experienced decline in PSA from baseline as best response, including a 56\% decline in PSA following two weeks of therapy seen in Patient \#3 (baseline and 2 week post-PSA values of 33.67 and $14.84 \mathrm{ng} / \mathrm{dL}$, respectively) (Figure 3). Patient \#1 maintained a stable PSA close to baseline measurements on enzalutamide therapy for 7 weeks after discontinuing NLG207, with baseline and 11-week post-baseline PSA measurements of 51.08 and $53.57 \mathrm{ng} / \mathrm{dL}$, respectively; Patient \#1's lowest PSA value was $43.75 \mathrm{ng} / \mathrm{dL}$, indicating a 14\% PSA decline as best response. At the time of analysis, samples collected for correlative analyses have not been evaluated. The quantitation of the PK samples has been incorporated into the dataset used for the population PK analysis of NLG207 (Chapter 6). Non-compartmental analysis of PK, urine sample analysis, and PD biomarker analysis are planned following continued accrual.

\section{Discussion}

Accrual to the present study has been limited, thus it is difficult to adequately draw any reasonable conclusions. Many factors have contributed to difficulty in accrual, notably the recent COVID-19 pandemic, multiple FDA-approved therapies available to patients with $\mathrm{mCRPC},{ }^{50}$ and a wide variety of clinical trials ongoing in treatment refractory mCRPC, including two competing studies at the National Cancer Institute (NCT02769962, NCT02484404). Administration of NLG207 every two weeks with 4 
hours total of hydration in addition to a 1-hour infusion may not be desirable to patients, especially if less intensive once monthly infusions and outpatient oral regimens are available. Generally, patients with features of more advanced disease (e.g., unfavorable performance status, low baseline hematology panels, and high alkaline phosphatase levels) and who have received numerous lines of therapy are less likely to enroll onto clinical studies due to potentially strict inclusion/exclusion criteria, travel capabilities, and performance status. As described by Caffo et al. in 2015, response rates and survival diminished over successive lines of therapy, and the number of patients receiving $4^{\text {th }}$ line treatment in the retrospective study was far less than in the $3^{\text {rd }}$ line (38 vs. 260 patients, respectively). ${ }^{41}$ The patients accrued to the study so far are representative of the potentially eligible population, having already received $4+$ lines of therapy for mCRPC treatment, with the majority of therapies being standard of care options. Though all patients presented with an ECOG performance status of 1, two of 3 patients had fluctuating red blood cell (RBC) and ANC counts from baseline throughout treatment and one patient had a significantly elevated alkaline phosphatase. Such patient characteristics may have complicated our ability to evaluate the optimal NLG207 dose in combination with enzalutamide via intra-patient dose escalation; a long 3-month DLT period required to ensure steady-state enzalutamide concentrations (plasma half-life of 5.8 days $)^{51}$ is also not ideal.

Adverse events possibly related to NLG207 were generally manageable. The reported Grade 2 non-infective cystitis and associated hematuria in Patient \#1 resulted in the first DLT associated with $12 \mathrm{mg} / \mathrm{m}^{2}$ dose of NLG207 thus enrollment in the dose escalation phase will require 6 patients to be enrolled before proceeding to the phase II 
portion of the study. Non-infective cystitis and hematuria of any grade are well known toxicities associated with NLG207 treatment, and were reported in $27 \%$ and $18 \%$ of patients, respectively, in the first-in-human study. ${ }^{36}$ Bladder-associated toxicity has been reported in numerous phase II studies that followed also. ${ }^{32-36}$ Trough concentrations in Patient \#1 showed that there are still low levels of camptothecin, both free and bound to the nanoparticle formulation, present in plasma 15 days post-treatment with the 12 $\mathrm{mg} / \mathrm{m}^{2}$ dose. The persistence of detectable plasma camptothecin concentrations further demonstrates the ability of the formulation to retain drug in the body long after dose; it also highlights the potential for toxicity, as Patient \#1 did not report the AE until 10 days after the last NLG207 infusion, well after the distribution phase following NLG207 infusion (Chapter 6). ${ }^{36}$ Hydration and frequent voiding are established as the key mitigation strategy for toxicity, especially during the first 48 hours post-infusion, primarily to limit accumulation of camptothecin in the bladder. ${ }^{36}$ Our findings suggest the importance of counseling patients about adequate hydration throughout the entire dosing interval to mitigate toxicity. It's worth noting that following the presentation of non-infectious cystitis in Patient \#1, the use of sodium bicarbonate to alkalinize urine did not improve symptoms, however, its role in prophylaxis remains a key correlative study worthy of investigation.

Though the duration of treatment has been severely limited, encouraging PSA responses have been observed on study so far, including one patient with a $>50 \%$ PSA decline following one dose of NLG207 and another patient with stable PSA on-study. Further robust enrollment is needed to evaluate the clinical anti-tumor activity of the 
combination and the associated response with respect to relevant pharmacodynamic biomarkers of acquired resistance.

\section{Acknowledgements}

We thank the nursing staff of National Cancer Institute and the fellows of the Genitourinary Malignancies Branch at National Cancer Institute for their care of our patients. Most importantly, we appreciate the patients with cancer who enroll in investigational trials to advance the knowledge of this disease.

\section{Ethics approval and consent to participate:}

The trial was approved by the institutional review board of the Center for Cancer Research, National Cancer Institute (ClinicalTrials.gov identifier: NCT03531827). Written informed consent was obtained for all patients prior to performing study-related procedures in accordance with federal and institutional guidelines.

\section{Funding}

NLG207 was provided by NewLink Genetics and Enzalutamide was purchased commercially. This project has been funded in whole or in part with federal funds from the National Cancer Institute, National Institutes of Health, under Contract No.

HHSN261200800001E. The content of this publication does not necessarily reflect the views or policies of the Department of Health and Human Services, nor does mention of trade names, commercial products, or organizations imply endorsement by the U.S. Government. 


\section{Grant Support}

This work was supported by the Intramural Research Program of the Center for Cancer

Research, National Cancer Institute, National Institutes of Health. 


\section{REFERENCES}

1. Beer TM, Armstrong AJ, Rathkopf DE, et al. Enzalutamide in metastatic prostate cancer before chemotherapy. N Engl J Med 2014;371:424-33.

2. $\quad$ Scher HI, Fizazi K, Saad F, et al. Increased survival with enzalutamide in prostate cancer after chemotherapy. N Engl J Med 2012;367:1187-97.

3. Hussain M, Fizazi K, Saad F, et al. Enzalutamide in Men with Nonmetastatic, Castration-Resistant Prostate Cancer. N Engl J Med 2018;378:2465-74.

4. Armstrong AJ, Szmulewitz RZ, Petrylak DP, et al. ARCHES: A Randomized, Phase III Study of Androgen Deprivation Therapy With Enzalutamide or Placebo in Men With Metastatic Hormone-Sensitive Prostate Cancer. J Clin Oncol 2019;37:297486.

5. Davis ID, Martin AJ, Stockler MR, et al. Enzalutamide with Standard First-Line Therapy in Metastatic Prostate Cancer. N Engl J Med 2019;381:121-31.

6. Chi KN, Agarwal N, Bjartell A, et al. Apalutamide for Metastatic, CastrationSensitive Prostate Cancer. N Engl J Med 2019;381:13-24.

7. Smith MR, Saad F, Chowdhury S, et al. Apalutamide Treatment and Metastasisfree Survival in Prostate Cancer. N Engl J Med 2018;378:1408-18.

8. Robinson D, Van Allen EM, Wu YM, et al. Integrative clinical genomics of advanced prostate cancer. Cell 2015;161:1215-28.

9. Linder S, van der Poel HG, Bergman AM, Zwart W, Prekovic S. Enzalutamide therapy for advanced prostate cancer: efficacy, resistance and beyond. Endocr Relat Cancer 2018;26:R31-R52.

10. Annala M, Vandekerkhove G, Khalaf D, et al. Circulating Tumor DNA Genomics Correlate with Resistance to Abiraterone and Enzalutamide in Prostate Cancer. Cancer Discov 2018;8:444-57.

11. Balbas MD, Evans MJ, Hosfield DJ, et al. Overcoming mutation-based resistance to antiandrogens with rational drug design. Elife 2013;2:e0499.

12. Azad AA, Volik SV, Wyatt AW, et al. Androgen Receptor Gene Aberrations in Circulating Cell-Free DNA: Biomarkers of Therapeutic Resistance in CastrationResistant Prostate Cancer. Clin Cancer Res 2015;21:2315-24.

13. Joseph JD, Lu N, Qian J, et al. A clinically relevant androgen receptor mutation confers resistance to second-generation antiandrogens enzalutamide and ARN-509.

Cancer Discov 2013;3:1020-9.

14. Antonarakis ES, $\mathrm{Lu} \mathrm{C}$, Wang $\mathrm{H}$, et al. AR-V7 and resistance to enzalutamide and abiraterone in prostate cancer. N Engl J Med 2014;371:1028-38.

15. Antonarakis ES, Lu C, Luber B, et al. Androgen Receptor Splice Variant 7 and Efficacy of Taxane Chemotherapy in Patients With Metastatic Castration-Resistant Prostate Cancer. JAMA Oncol 2015;1:582-91.

16. Clark E, Morton M, Sharma S, et al. Prostate cancer androgen receptor splice variant 7 biomarker study - a multicentre randomised feasibility trial of biomarkerguided personalised treatment in patients with advanced prostate cancer (the VARIANT trial) study protocol. BMJ Open 2019;9:e034708.

17. Fernandez EV, Reece KM, Ley AM, et al. Dual targeting of the androgen receptor and hypoxia-inducible factor 1alpha pathways synergistically inhibits castration-resistant prostate cancer cells. Mol Pharmacol 2015;87:1006-12. 
18. Bharti SK, Kakkad S, Danhier P, et al. Hypoxia Patterns in Primary and Metastatic Prostate Cancer Environments. Neoplasia 2019;21:239-46.

19. Fraga A, Ribeiro R, Principe P, Lopes C, Medeiros R. Hypoxia and Prostate Cancer Aggressiveness: A Tale With Many Endings. Clin Genitourin Cancer 2015; 13:295-301.

20. Stewart GD, Ross JA, McLaren DB, Parker CC, Habib FK, Riddick AC. The relevance of a hypoxic tumour microenvironment in prostate cancer. BJU Int 2010;105:8-13.

21. Schito L, Semenza GL. Hypoxia-Inducible Factors: Master Regulators of Cancer Progression. Trends Cancer 2016;2:758-70.

22. Semenza GL. Defining the role of hypoxia-inducible factor 1 in cancer biology and therapeutics. Oncogene 2010;29:625-34.

23. Park C, Kim Y, Shim M, Lee Y. Hypoxia enhances ligand-occupied androgen receptor activity. Biochem Biophys Res Commun 2012;418:319-23.

24. Mitani T, Yamaji R, Higashimura Y, Harada N, Nakano Y, Inui H. Hypoxia enhances transcriptional activity of androgen receptor through hypoxia-inducible factor1alpha in a low androgen environment. J Steroid Biochem Mol Biol 2011;123:58-64.

25. Mitani T, Harada N, Nakano Y, Inui H, Yamaji R. Coordinated action of hypoxia-inducible factor-1alpha and beta-catenin in androgen receptor signaling. J Biol Chem 2012;287:33594-606.

26. Schmidt KT, Peer CJ, Huitema ADR, et al. Measurement of NLG207 (formerly CRLX101) nanoparticle-bound and released camptothecin in human plasma. J Pharm Biomed Anal 2020;181:113073.

27. Lin CJ, Lin YL, Luh F, Yen Y, Chen RM. Preclinical effects of CRLX101, an investigational camptothecin-containing nanoparticle drug conjugate, on treating glioblastoma multiforme via apoptosis and antiangiogenesis. Oncotarget 2016;7:4240821.

28. Conley SJ, Baker TL, Burnett JP, et al. CRLX101, an investigational camptothecin-containing nanoparticle-drug conjugate, targets cancer stem cells and impedes resistance to antiangiogenic therapy in mouse models of breast cancer. Breast Cancer Res Treat 2015;150:559-67.

29. Pham E, Birrer MJ, Eliasof S, et al. Translational impact of nanoparticle-drug conjugate CRLX101 with or without bevacizumab in advanced ovarian cancer. Clin Cancer Res 2015;21:808-18.

30. Pham E, Yin M, Peters CG, et al. Preclinical Efficacy of Bevacizumab with CRLX101, an Investigational Nanoparticle-Drug Conjugate, in Treatment of Metastatic Triple-Negative Breast Cancer. Cancer Res 2016;76:4493-503.

31. Fruehauf JP, Farrokhian N, Sarkissian S, Kim JH. Blockade of ARV7:

HIF1alpha heterodimers after topotecan reverses enzalutamide resistance in 22Rv1 cells. J Clin Oncol 2016;34(15_suppl):e16594.

32. Chao J, Lin J, Frankel P, et al. Pilot trial of CRLX101 in patients with advanced, chemotherapy-refractory gastroesophageal cancer. J Gastrointest Oncol 2017;8:962-9.

33. Keefe SM, Hoffman-Censits J, Cohen RB, et al. Efficacy of the nanoparticledrug conjugate CRLX101 in combination with bevacizumab in metastatic renal cell carcinoma: results of an investigator-initiated phase I-IIa clinical trial. Ann Oncol 2016;27:1579-85. 
34. Sanoff HK, Moon DH, Moore DT, et al. Phase I/II trial of nano-camptothecin CRLX101 with capecitabine and radiotherapy as neoadjuvant treatment for locally advanced rectal cancer. Nanomedicine 2019;18:189-95.

35. Voss MH, Hussain A, Vogelzang N, et al. A randomized phase II trial of CRLX101 in combination with bevacizumab versus standard of care in patients with advanced renal cell carcinoma. Ann Oncol 2017;28:2754-60.

36. Weiss GJ, Chao J, Neidhart JD, et al. First-in-human phase 1/2a trial of CRLX101, a cyclodextrin-containing polymer-camptothecin nanopharmaceutical in patients with advanced solid tumor malignancies. Invest New Drugs 2013;31:986-1000. 37. Davis ME. Design and development of IT-101, a cyclodextrin-containing polymer conjugate of camptothecin. Adv Drug Deliv Rev 2009;61:1189-92.

38. Clark AJ, Wiley DT, Zuckerman JE, et al. CRLX101 nanoparticles localize in human tumors and not in adjacent, nonneoplastic tissue after intravenous dosing. Proc Natl Acad Sci U S A 2016;113:3850-4.

39. Chen YF, Wang YH, Lei CS, Changou CA, Davis ME, Yen Y. Host immune response to anti-cancer camptothecin conjugated cyclodextrin-based polymers. J Biomed Sci 2019;26:85.

40. Gaur S, Wang Y, Kretzner L, et al. Pharmacodynamic and pharmacogenomic study of the nanoparticle conjugate of camptothecin CRLX101 for the treatment of cancer. Nanomedicine 2014;10:1477-86.

41. Caffo O, De Giorgi U, Fratino L, et al. Clinical Outcomes of Castration-resistant Prostate Cancer Treatments Administered as Third or Fourth Line Following Failure of Docetaxel and Other Second-line Treatment: Results of an Italian Multicentre Study. Eur Urol 2015;68:147-53.

42. Schwartz LH, Litiere S, de Vries E, et al. RECIST 1.1-Update and clarification: From the RECIST committee. Eur J Cancer 2016;62:132-7.

43. Scher HI, Morris MJ, Stadler WM, et al. Trial Design and Objectives for Castration-Resistant Prostate Cancer: Updated Recommendations From the Prostate Cancer Clinical Trials Working Group 3. J Clin Oncol 2016;34:1402-18.

44. Cohen B, Laish I, Brosh-Nissimov T, et al. Efficacy of urine alkalinization by oral administration of sodium bicarbonate: a prospective open-label trial. Am J Emerg Med 2013;31:1703-6.

45. Vali AM, Shafaghi B, Dadashzadeh S. Simple and sensitive high performance liquid chromatographic method for the simultaneous quantitation of the lactone and carboxylate forms of topotecan in human plasma. J Chromatogr B Analyt Technol Biomed Life Sci 2005;818:205-12.

46. Greene SB, Dago AE, Leitz LJ, et al. Chromosomal Instability Estimation Based on Next Generation Sequencing and Single Cell Genome Wide Copy Number Variation Analysis. PLoS One 2016;11:e0165089.

47. Armstrong AJ, Halabi S, Luo J, et al. Prospective Multicenter Validation of Androgen Receptor Splice Variant 7 and Hormone Therapy Resistance in High-Risk Castration-Resistant Prostate Cancer: The PROPHECY Study. J Clin Oncol 2019;37:1120-9.

48. Kummar S, Gutierrez ME, Chen A, et al. Phase I trial of vandetanib and bevacizumab evaluating the VEGF and EGF signal transduction pathways in adults with solid tumours and lymphomas. Eur J Cancer 2011;47:997-1005. 
49. de Haan R, Pluim D, van Triest B, et al. Improved pharmacodynamic (PD) assessment of low dose PARP inhibitor PD activity for radiotherapy and chemotherapy combination trials. Radiother Oncol 2018;126:443-9.

50. Nuhn P, De Bono JS, Fizazi K, et al. Update on Systemic Prostate Cancer Therapies: Management of Metastatic Castration-resistant Prostate Cancer in the Era of Precision Oncology. Eur Urol 2019;75:88-99.

51. Gibbons JA, Ouatas T, Krauwinkel W, et al. Clinical Pharmacokinetic Studies of Enzalutamide. Clin Pharmacokinet 2015;54:1043-55. 


\section{FIGURES and TABLES}

\section{Figure 1. Study Overview}

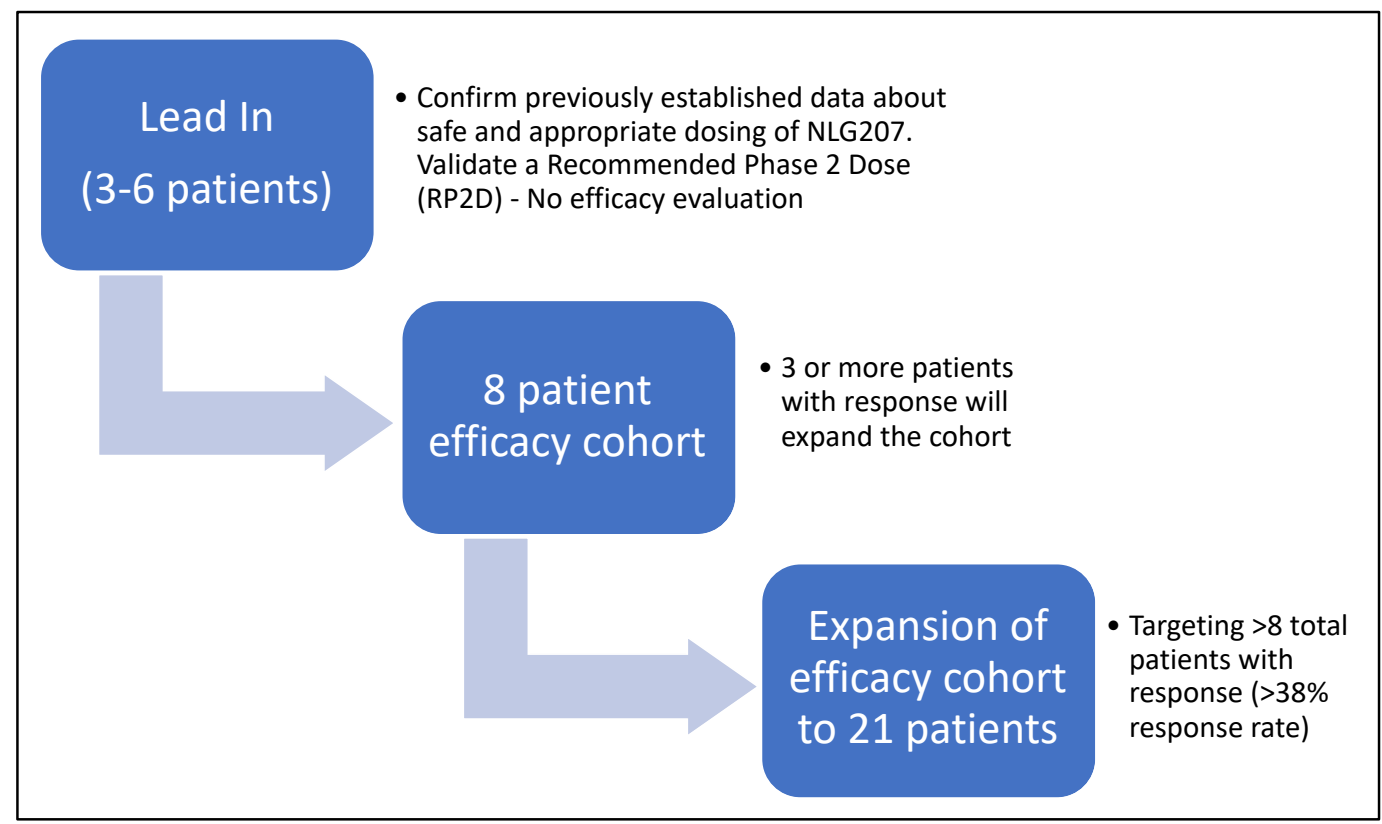


Figure 2. Dosing strategies
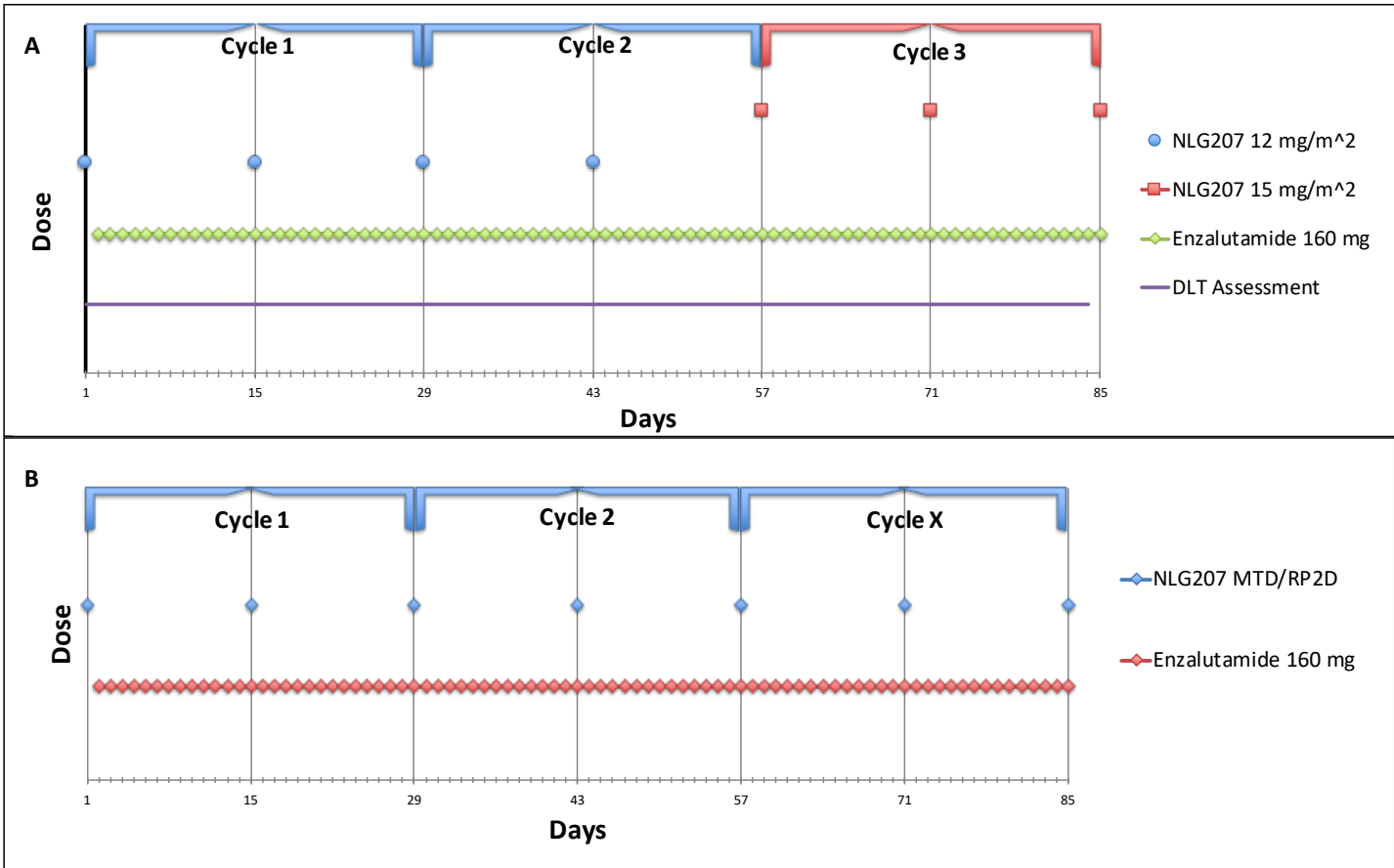

The dosing schema for the lead-in dose escalation with DLT assessment (A) and for the remainder of the study following confirmation of the MTD/RP2D (B). 
Figure 3. Best PSA Response in Patients Treated with NLG207 and Enzalutamide.

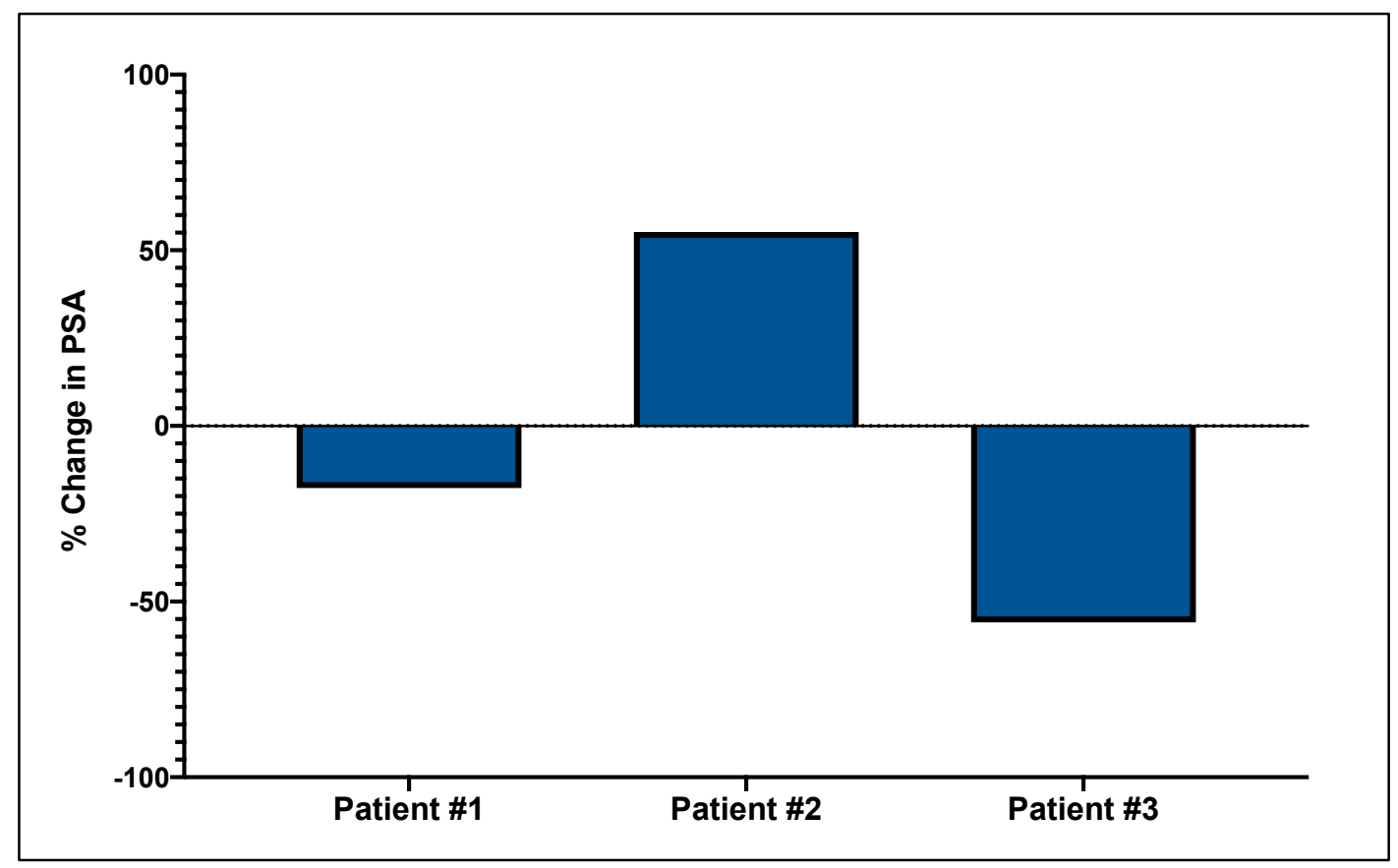


Table 1. Baseline Patient Characteristics

\begin{tabular}{|c|c|c|c|}
\hline & Patient \#1 & Patient \#2 & Patient \#3 \\
\hline Age $(y)$ & 72 & 69 & 76 \\
\hline Race & African American & Caucasian & Caucasian \\
\hline Weight (kg) & 70.4 & 71.6 & 78.2 \\
\hline ECOG performance status & 1 & 1 & 1 \\
\hline On-study PSA (ng/dL) & 51.08 & 143 & 33.67 \\
\hline Site(s) of metastasis & Bone & Bone & $\begin{array}{l}\text { Bone, liver, lymph } \\
\text { node }\end{array}$ \\
\hline Gleason Score at Diagnosis & 9 & 8 & $\mathrm{~N} / \mathrm{A}$ \\
\hline $\begin{array}{l}\text { Prior Treatments (includes } \\
\text { Enzalutamide) }\end{array}$ & $\begin{array}{c}\text { Radium-223 } \\
\text { Docetaxel } \\
\text { Abiraterone Acetate }\end{array}$ & $\begin{array}{l}\text { Abiraterone Acetate } \\
\text { Cabazitaxel } \\
\text { Carboplatin/Docetaxel } \\
\text { Durvalumab/olaparib }\end{array}$ & $\begin{array}{c}\text { Docetaxel } \\
\text { Abiraterone Acetate } \\
\text { Carboplatin }\end{array}$ \\
\hline
\end{tabular}


Chapter 8

Summary 
The presented thesis work aims to address the development of novel therapeutic approaches to combat acquired resistance to second-generation androgen receptor antagonists (SG-ARAs) in patients with advanced prostate cancer. The scope of this work includes an extensive review of the literature, the analysis of raw clinical outcomes from a phase 2 study, the application of preclinical models to support proposed clinical anti-tumor activity, the validation of bioanalytical methods for pharmacokinetic studies, the development of a population pharmacokinetic model, and the design of a rationalebased, biomarker-driven clinical trial.

First, Chapter 2 provides a literature review of important clinical trials that have established the role of SG-ARA therapy in patients with advanced prostate cancer.

Clinically relevant biomarkers are summarized, including AR amplification, RB1 and TP53 loss, and germline DNA damage repair (DDR) mutations, highlighting the capability to detect actionable pathways of acquired resistance in patients and ongoing efforts to employ precision medicine into prostate cancer management. Completed and active clinical trials of importance to mitigating poor outcomes from SG-ARA therapy were also discussed.

In Chapter 3, clinical outcomes from a phase 2 study administering seviteronel, an investigational next-generation CYP17-lyase inhibitor, to patients with mCRPC following disease progression on enzalutamide were evaluated. Despite a proposed affinity to the AR with point mutations conferring resistance to enzalutamide, seviteronel failed to improve clinical outcomes in a heavily pre-treated population. Not only did the study fail to generate a favorable PSA response rate, but also a majority of the patients experienced intolerable and treatment limiting CNS-related toxicity. Non- 
compartmental analysis of pharmacokinetics yielded a longer half-life than anticipated, which led to significant accumulation of drug using the implemented dosing strategies and thus supported the observed toxicity profile. The results from this study also emphasize the limitations of repetitive lines of AR-targeted therapies at later stages of disease; ultimately, the observed response rate of seviteronel was similar to that of abiraterone acetate in an enzalutamide pre-treated population, demonstrating no significant gain with the optimized mechanism of action previously described in preclinical studies.

In response to the negative clinical trial of seviteronel, Chapter 4 proposed targeting alternative metabolic signaling up-regulated in hypoxic tumors to overcome enzalutamide resistance. Previous laboratory generated data demonstrated the downregulation of HIF- $1 \alpha$ via siRNA silencing could re-sensitize AR-V7 expressing cells (22Rv1) to enzalutamide. Intramural NCI research showed low persistent doses of topotecan, a camptothecin-derived topoisomerase I inhibitor, down-regulate HIF-1 $\alpha$ protein accumulation, a finding that was verified clinically via a biomarker-driven study in patients with advanced solid tumors. NLG207, a nanoparticle-drug conjugate formulation used to facilitate the delivery of camptothecin (CPT), was shown to similarly down-regulate HIF- $1 \alpha$ in preclinical models and induce down-regulation of HIF-1 $\alpha$-target genes in clinical tumor specimens. Thus, NLG207 in combination with enzalutamide was hypothesized to have in vivo anti-tumor activity via dual HIF-1 $\alpha$ and AR inhibition. NLG207 in combination with enzalutamide showed significant antitumor activity in preclinical prostate cancer xenograft models of AR-V7 expression and AR-FL overexpression (22Rv1 and castrated VCaP, respectively), both relevant 
acquired resistance mechanisms to enzalutamide; tumors were also re-sensitized to enzalutamide following treatment with NLG207, as evidenced by the analysis of tumor growth in castrated mice with $\mathrm{VCaP}$ xenografts. Correlative in vitro analysis confirmed the in vivo findings, showing NLG207 drove down AR-V7 expression and enhanced down-regulation of AR-target gene expression (KLK3, ERG) when in combination with enzalutamide. The preclinical data provided sufficient rationale to investigate NLG207 and enzalutamide in patients with mCRPC following disease progression on enzalutamide.

To enable clinical pharmacokinetic analysis of investigational NLG207, Chapter 5 describes the development of a novel, robust, selective, accurate and precise bioanalytical method to quantify CPT concentrations, both bound and unbound to NLG207. The UHPLC-MS/MS-based assay was used to evaluate the stability of nanoparticles in clinical samples, supporting the previously established sample processing methodology. The assay was also shown to be clinically applicable, as demonstrated via the quantitation of patient plasma samples and subsequent generation of individual plasma concentration-time curves. Using the assay to quantitate CPT concentrations from ongoing NLG207 clinical studies, a population pharmacokinetic model was developed in Chapter 6. To best describe NLG207 pharmacokinetics, a model combining two linear two-compartment models with first-order kinetics each to describe bound and free CPT with body weight based allometric scaling on parameters was used. Nanoparticle behavior of the formulation was confirmed via small volumes of distribution for the bound CPT compartments, consistent with containment of camptothecin in the soluble delivery vehicle and tumor uptake dictated by the enhanced 
permeation and retention (EPR) effect. Importantly, the model characterized CPT release in plasma via an initial rapid clearance rate that decreased over the first 4 hours post-infusion to a steady-state clearance, a deviation from previous models characterizing liposomal nanoparticle formulations. With incorporation of larger datasets, the model may prove to be a useful tool to predict clinical outcomes and toxicity.

With important analytical and pharmacokinetic tools established in Chapters 5 and 6, and preclinical data generated in Chapter 4, a clinical trial to evaluate NLG207 in combination with enzalutamide in patients with mCRPC previously progressing on enzalutamide was initiated, as described in Chapter 7. The primary objective of the ongoing study is to evaluate anti-tumor activity with respect to PSA reduction and/or stable disease on radiographic imaging, following the validation of the recommended phase 2 dose of NLG207 when in combination with enzalutamide. To date, limited enrollment prevents adequate interpretation of the tolerability and efficacy of the treatment combination in this heavily pre-treated population. Early data from 3 patients, however, suggests some clinical activity with respect to PSA response. Only one patient was evaluable for toxicity, to which the patient experienced Grade 2 non-infective cystitis and hematuria, resulting in a dose-limiting toxicity (DLT) following lack of resolution after two weeks of holding NLG207. To better understand the DLT, near realtime quantitation and analysis of CPT concentrations from clinical samples drawn was made feasible via the developed bioanalytical methods. Additionally, the cycle 1 pharmacokinetic data obtained from these three patients were incorporated into the population pharmacokinetic model to further aid mechanistic interpretation of the 
NLG207 formulation. The challenges of enrolling and successfully treating patients with advanced mCRPC are evident from early trial experience. Nevertheless, enrollment is ongoing with planned analysis of clinical outcomes and clinical response with respect to a panel of pharmacodynamic biomarkers.

Overall, this thesis aimed to assess novel therapeutic approaches to overcome relevant mechanisms of SG-ARA acquired resistance in patients with advanced prostate cancer. The data generated from this work and studies to follow will greatly contribute to the understanding of SG-ARA acquired resistance and the further optimization of treatment for patients with advanced prostate cancer. 


\section{Hoofdstuk 8}

Samenvatting 
In dit proefschrift worden nieuwe benaderingen beschreven voor de behandeling van patiënten met prostaatkanker met verworven resistentie op tweede generatie androgeen receptor (AR) antagonisten (SG-ARAs). Dit proefschrift bestaat uit een overzicht van de literatuur, resultaten van een fase II klinische studie, toepassing van preklinische modellen, ontwikkeling en validatie van bioanalytische bepalingsmethodes voor farmacokinetische studies, de ontwikkeling van een populatie farmacokinetiek model en de opzet van klinische studies gebaseerd op rationele biomarkers.

Hoofdstuk 2 van dit proefschrift geeft een overzicht van de belangrijke klinische studies die hebben geleid tot de toepassing van SG-ARAs in de behandeling van prostaatkanker. Verschillende biomarkers voor uitkomst, zoals AR amplificatie, verlies van RB1 en TP53 en mutaties in genen betrokken bij DNA reparatie, zijn beschreven en kunnen gebruikt worden om de behandeling verder te verbeteren uiteindelijk leidend tot verdere toepassing van precision medicine bij de behandeling van prostaatkanker.

In Hoofstuk 3 worden de uitkomsten van een fase 2 studie naar seviteronel, een nieuwe CYP17-lyase remmer, bij prostaatkanker patiënten beschreven. De rationale voor de studie was dat seviteronel mogelijk actief is bij prostaatkanker resistent voor enzalutamide. Helaas bleek in deze fase II studie geen voordeel van de behandeling met seviteronel terwijl ernstige bijwerkingen optraden. In de farmacokinetische analyse van de data bleek seviteronel een langere halfwaardetijd te hebben dan gedacht, waardoor er bij de gekozen doseringsstrategie accumulatie van seviteronel optrad. Dit kan bijgedragen hebben aan de opgetreden bijwerkingen. Deze studie laat ook de beperkingen zien van opeenvolgende lijnen van AR gerichte therapie. De geobserveerde response in deze studie op seviteronel was vergelijkbaar als abiraterone en daarmee is er 
geen voordeel van seviteronel te verwachten in tegenstelling tot wat de resultaten van preklinisch onderzoek suggereerden.

In Hoofdstuk 4 wordt een preklinische studie beschreven om nieuwe aangrijpingspunten voor therapie in vergevorderde prostaatkanker resistent tegen enzalutamide te vinden. Deze studie werd uitgevoerd mede naar aanleiding van de negatieve resultaten van de seviteronel studie beschreven in Hoofdstuk 3, wat eens te meer duidelijk maakte dat nieuwe aangrijpingspunten voor de behandeling van prostaatkanker dringend gewenst zijn. In eerder onderzoek is gebleken dat regulatie van HIF-1 $\alpha$ expressie door middel van siRNA silencing AR-V7 cellen opnieuw gevoelig kan maken voor enzalutamide. Ook was aangetoond dat langdurige blootstelling aan lage doses topotecan, een camptothecine topoisomerase I remmer, zorgt voor down regulatie van HIF-1 $\alpha$. NLG207 is een nanopartikel formulering van camptothecine. In een preklinische studie is aangetoond dat NLG207 op een zelfde wijze kan zorgen voor vermindering van HIF- $1 \alpha$. In dit hoofdstuk werd aangetoond dat de combinatie van NLG207 met enzalutamide een antitumor effect heeft in xenograft modellen met cellijnen resistent voor enzalutamide monotherapie (22Rv1 en $\mathrm{VCaP})$. Hierbij werd aangetoond dat NLG207 zorgt voor lagere expressie van AR-V7 en down regulatie van AR. Deze data vormden de rationale voor het opzetten van een klinische studie naar deze combinatie bij patiënten met ziekteprogressie op behandeling met enzalutamide.

Voor het uitvoeren van de klinische studie met NLG207 is het nodig om een gevoelige en gevalideerde methode te hebben voor de bepaling van NLG207 in plasma. Om de farmacokinetiek van NLG207 goed te kunnen bestuderen, moeten zowel de gebonden camptothecine als de vrije fractie gekwantificeerd worden. In Hoofdstuk 5 
wordt de ontwikkeling en validatie van deze bepalingsmethode met behulp van UHPLCMS/MS beschreven. De stabiliteit van NLG207 na afname is belangrijk om zowel gebonden als vrij camptothecine te kunnen meten in materiaal van patiënten. De ontwikkelde bepalingsmethode bleek goed geschikt voor het meten van NLG207 in dit materiaal en werd uitgebreid gevalideerd.

In Hoofstuk 6 is de ontwikkeling van een populatie farmacokinetiek model voor zowel vrij als gebonden camptothecine na toediening van NLG207 beschreven. Het uiteindelijke model bestond uit een twee compartimentsmodel voor zowel gebonden als vrij camptothecine. Lichaamsgewicht was gerelateerd aan alle farmacokinetische parameters door middel van allometrische schaling. Gebonden camptothecine had een klein verdelingsvolume zoals te verwachten van de nanopartikel formulering. Het vrijkomen van camptothecine uit deze formulering was beschreven door middel van een snelle initieel proces gedurende ongeveer 4 uur, waarna een veel langzamere klaring ontstond op latere tijdspunten, leidend tot een vrij lange blootstelling aan lage concentraties camptothecine.

Op basis van de preklinische data beschreven in Hoofdstuk 4 en met de beschikbaarheid van een bioanalytische bepalingsmethode (Hoofdstuk 5) en een farmacokinetisch model (Hoofdstuk 6) werd een klinische studie gestart naar de combinatie van NLG207 en enzalutamide in prostaatkanker patiënten na falen van enzalutamide. De opzet en interim resultaten van deze studie zijn beschreven in Hoofdstuk 7. Het primaire doel van deze studie is het vaststellen van de antitumor activiteit van deze combinatie (zowel op basis van PSA afname als op basis van beeldvorming van de tumor) nadat de juiste dosis van de combinatie is vastgesteld. 
Inclusie in deze studie bleek moeilijk onder andere doordat patiënten al een aantal lijnen van behandeling hadden gehad voor ze voor deze studie in aanmerking kwamen. Door de beperkte inclusie op het moment van deze interimanalyse is een adequate interpretatie van bijwerkingen en effectiviteit nog niet mogelijk. Op het moment van analyse waren drie patiënten geïncludeerd in de studie en de data suggereren enige antitumor activiteit. Slechts één patiënt was evalueerbaar voor bijwerkingen. Deze patiënt had een graad 2 bijwerkingen van een niet-infectieuze blaasontsteking en dit werd gezien als een dosis limiterende bijwerking. De eerder beschreven analysemethode is gebruikt om de farmacokinetische data van NLG207 te analyseren in deze patiënten. Alhoewel inclusie van patiënten in deze studie lastig bleek, is de studie nog open voor inclusie.

In dit proefschrift worden nieuwe benaderingen voor de behandeling van prostaatkanker resistent voor bestaande opties beschreven. De studies beschreven in dit proefschrift hebben bijgedragen aan ons begrip van resistentie op SG-ARAs en helpen bij het verder verbeteren van de behandeling van deze patiënten. 


\section{Chapter 9}

\section{Discussion}

Key Over-arching Questions Addressed:

1. Can meaningful clinical outcomes be achieved via further enhanced AR-targeted therapies in patients with mCRPC previously treated with enzalutamide?

2. Can an efficient HIF-1 $\alpha$-targeted approach be used to down-regulate mechanisms of enzalutamide resistance and re-sensitize tumors to enzalutamide?

3. How do co-polymer-based nanoparticles impact pharmacokinetics and drug delivery in comparison to conventional formulations and alternative nanoparticle formulations?

4. Is co-targeting HIF-1 $\alpha$ and the AR a feasible approach to improve clinical outcomes in patients with mCRPC previously treated with enzalutamide? 
Overcoming acquired resistance to enzalutamide and other second-generation androgen receptor antagonists (SG-ARAs) in patients with advanced prostate cancer remains an unmet clinical need. Numerous mechanisms of acquired resistance have been elucidated, uncovering both clinically relevant biomarkers and druggable targets worthy of clinical development. In addition to ongoing treatment paradigm optimization, clinical research efforts have focused on newer therapeutic modalities and treatment combinations, including those that differentially inhibit the androgen receptor pathway and down-regulate metabolic signaling pathways associated with increased intra-tumoral hypoxia. Critical to ongoing research efforts is the incorporation of translational research approaches, utilizing lessons learned in the clinic and bringing them back into the laboratory for further examination, before ultimately refining new hypotheses for follow-up rationale-based clinical trial investigation. The work presented in this thesis focuses on the development of rationale-based novel therapeutics to overcome resistance to SG-ARA therapy prevalent in patients with advanced prostate cancer.

The investigation of seviteronel in patients with mCRPC sought to boldly target weaknesses of monotherapy with enzalutamide and abiraterone acetate, seeking optimized clinical outcomes in the setting of acquired resistance via re-targeting of the AR. Critical to seviteronel's activity was specificity to CYP17 lyase over hydroxylase functionality, potentially limiting the necessity of co-administered prednisone associated with abiraterone treatment. The toxicity profile and inability to find an optimal dosing strategy for seviteronel nixed this potential niche for the agent. The drug also targeted point mutations commonly observed in the context of enzalutamide resistance (i.e. T877A, F877L), leading to the proposed benefit in patients with prior enzalutamide 
therapy. Hindered greatly from toxicity, the efficacy analysis did not demonstrate a meaningful response rate, but rather suggested no difference from abiraterone acetate in a similar setting (Chapter 3). Phase III trials investigating orteronel, a similar CYP17lyase specific agent that required prednisone co-administration, failed to demonstrate significant overall survival benefits in patients with MCRPC without prior abiraterone or enzalutamide treatment. ${ }^{1}$ A regionally-stratified secondary analysis of the Phase III study of orteronel in patients with mCRPC post-docetaxel (ELM-PC-5) showed the incorporation of enzalutamide and abiraterone as follow-up lines of therapy, occurring frequently in Europe and North America, greatly reduced overall survival, suggestive of over-targeting the AR. ${ }^{1}$ Possibly cognizant of this data, a Phase III trial of galeterone, another potent CYP17-lyase inhibitor, sought to treat patients with AR-V7-positive, treatment-naïve mCRPC, to which the investigators terminated the study early upon realizing such a population was impossible to accrue. ${ }^{2}$ Clinical investigation of darolutamide, an optimized AR antagonist with potency towards the F877L and T877A AR mutations, adapted to the existing treatment landscape via selecting patients earlier in the disease course (i.e. nmCRPC) to demonstrate efficacy. ${ }^{3}$ These data signify the proverbial ceiling of sole AR-targeting in pre-treated patients with MCRPC and the importance of tumor characterization to further optimize therapy.

Alterations to the AR and other pathways were shown to be diverse in patients with mCRPC, emphasizing the importance of understanding tumor heterogeneity. AR point mutations, notably the F877L mutation shown to promote enzalutamide agonist activity in vitro, were not observed frequently in patients with disease progression on either enzalutamide or apalutamide, supporting the limited benefit of seviteronel in 
sequence with enzalutamide. AR amplification and AR-V7 expression occur more frequently by comparison in patients with MCRPC post-enzalutamide, justifying more refined approaches, including those targeting AR-V7 (e.g. EPI-506) (Chapter 2). Interestingly, significant heterogeneity of AR gene re-arrangements was shown to produce diverse splice variant expression and differential AR overexpression, demonstrating the complexity associated with AR pathway mediated enzalutamide resistance. ${ }^{4}$ Additional studies addressing tumor characteristics have emphasized targeting alternative metabolic pathways as opposed to further AR inhibition, including the PI3K/AKT, WNT/ $/$-catenin, DDR and cell cycle pathways. ${ }^{5}$ Appropriate and informed interpretation of clinical biomarkers is necessary for the development of new therapeutics, as highlighted by the recent approvals of olaparib and rucaparib (Chapter 2). Enhanced understanding of tumor biology and frequent oncogenic driving mechanisms are also of great significance.

Co-targeting HIF- $1 \alpha$ and the AR via siRNA-mediated HIF- $1 \alpha$ inhibition and enzalutamide, respectively, was shown to be a rational approach to target $\beta$-catenin pathway-mediated upregulation of angiogenesis and AR activity in enzalutamide resistant, AR-V7 expressing $22 \mathrm{Rv} 1$ cells in vitro. ${ }^{6}$ However, identifying a clinically applicable direct inhibitor of HIF-1 $\alpha$ with minimal residual activity presented a major challenge, as no such inhibitor has been approved for the treatment of patients with cancer due to limited safety or clinical activity. One direct inhibitor, ENZ-2968, a synthetic anti-sense oligonucleotide fully complementary to the mRNA sequence of HIF-1 $\alpha$, showed promising clinical activity but further development was suspended for undisclosed reasons. ${ }^{7,8}$ Targeting the interaction of HIF- $1 \alpha$ and $\mathrm{p} 300$ with chetomin was 
counterintuitively shown to increase AR transcriptional activation, likely via paninhibition of p300-containing transcriptional complexes. ${ }^{6}$ Though the anthracyclines, doxorubicin and daunorubicin, have been shown to inhibit binding of HIF-1 to hypoxia response elements (HREs), ${ }^{9}$ clinically significant cardiac toxicity negatively impacts the risk-benefit ratio and restricts the cumulative lifetime dose of these agents. ${ }^{10}$ Topotecan, a camptothecin-derived topoisomerase I inhibitor that indirectly inhibits HIF-1 $\alpha$ protein accumulation, was shown to reduce HIF-1 $\alpha$ protein concentrations in clinical tumor specimens. ${ }^{11}$ The nanoparticle-drug conjugate of camptothecin (CPT), NLG207, had also demonstrated evidence of HIF-1 $\alpha$ inhibition in both preclinical models and clinical tumor specimens, while also facilitating more optimal drug delivery in comparison to small molecule topotecan (Chapter 4). NLG207 was sufficient to interrogate inhibition of HIF-AR crosstalk despite potent topoisomerase I activity, however, the development a potent HIF-1 $\alpha$ specific inhibitor remains an important drug development objective with the potential to limit off-target toxicities of currently utilized investigational agents.

Preclinical evaluation of NLG207 and enzalutamide demonstrated significant anti-tumor activity in two in vivo models of enzalutamide resistance. A key limitation of the preclinical studies was the inability to interrogate the direct activity against HIF-1 $\alpha$, primarily due to the limited acquisition of sufficient tumor tissue for analysis. The mRNA expression analysis of down-stream targets, including VEGF and KLK3, established evidence of HIF-AR crosstalk inhibition in prior both prior in vitro studies and the current study in $\mathrm{VCaP}$ cells. Consistent with prior anti-tumor activity observed with camptothecin-based treatment in vitro, our findings demonstrated significant downregulation of AR-V7 and KLK3 transcription following combination NLG207 and 
enzalutamide treatment. In the castrated VCaP xenograft model, activity of NLG207 and enzalutamide was similar to that of JQ1, a bromodomain and extraterminal (BET) inhibitor shown to attenuate AR signaling, in combination with enzalutamide; notably, AR-V7 gene expression was down-regulated via treatment with either NLG207 or JQ1, enabling the enhancement of enzalutamide treatment and tumor growth reduction (Chapter 4). The preclinical data further supports treatment approaches targeting alternative signaling pathways that contribute to unattenuated AR signaling in SG-ARAresistant prostate cancer.

In addition to investigations of NLG207's anti-tumor activity, this thesis sought to better characterize the novel co-polymer-based nanoparticle formulation and understand clinical pharmacological differences in comparison to both conventional drug administration and approved liposomal nanoparticle formulations. NLG207 was designed to improve the poor physicochemical and pharmacokinetic (PK) properties of CPT, which prevented the safe and efficacious use of an agent with promising preclinical activity (Chapter $\mathbf{5} \& \mathbf{6}$ ). Irinotecan and topotecan, clinically approved derivatives of CPT, have increased solubility, but are still associated with clinically significant toxicity and suboptimal PK following administration via conventional formulations. ${ }^{12} \mathrm{CPT}$ was shown to more potently reduce HIF- $1 \alpha$ accumulation in comparison to topotecan, ${ }^{13}$ justifying the administration of CPT with optimized pharmaceutical characteristics. In the NLG207 formulation, subunits comprised of CPT conjugated in proximity to $\beta$-cyclodextrins are tethered together via polyethylene glycol repeats to form co-polymer units. Aqueous conditions then exploit the unique properties of $\beta$-cyclodextrin to interact with camptothecin while promoting hydrophilic interactions 
on the outer surfaces, enabling 4-5 co-polymer strands to fold into soluble nanoparticles with a diameter of 20-40 nm; by comparison, nanoparticles of approved liposomal formulations (e.g. doxorubicin, irinotecan) are approximately $100 \mathrm{~nm}$ in diameter. Studies to further characterize population pharmacokinetics (popPK) of NLG207 were sought and made possible via validated bioanalytical methods to quantitate nanoparticlebound and free CPT concentrations in clinical plasma samples (Chapter 5).

The NLG207 popPK model demonstrated a similar distribution pattern of nanoparticle-bound CPT to that of liposomal irinotecan, confirming the retention of camptothecin within the formulation when in the plasma circulation based on the small volume of distribution of the conjugated drug. Drug release from NLG207, governed by pH dependent hydrolysis, was described by an initial rapid release rate that gradually dissipated during the first 4 hours post-infusion, a finding that greatly differed from liposomal irinotecan. The rapid initial release is hypothesized to result from imperfections of nanoparticle folding, leaving exposed camptothecin on the surfaces to undergo hydrolysis upon introduction into the plasma (Chapter 6). Renal clearance of released CPT and nanoparticle-bound CPT, the latter unaccounted for in the popPK analysis, increase the potential for non-infective cystitis in the first 48 hours, leading to the use of pre- and post-dose hydration strategies; ${ }^{14}$ this hydration strategy is not required for approved liposomal nanoparticle formulations. Nevertheless, the NLG207 formulation significantly reduces systemic exposure of free CPT relative to nanoparticle-bound CPT (Chapter 6).

Though NLG207 improves the physicochemical and pharmacokinetic properties of camptothecin, mechanisms governing delivery into tumor tissue warrant optimization 
in subsequent generations of nanoformulation development. Size exclusion and the enhanced permeation and retention (EPR) effect largely dictate uptake into tumor tissue and are heavily reliant upon reduced immune surveillance and renal clearance to increase plasma retention time and facilitate entry into tumor tissue via typically larger cellular fenestrations. A by-product of prolonged plasma retention time likely includes accumulation in highly perfused tissues in addition to tumors, as suggested by the volume of distribution estimate of the peripheral compartment and prior preclinical PK analyses (Chapter 6). Despite prior clinical evidence of specific targeting of tumor tissue as opposed to adjacent healthy tissue,${ }^{15}$ the depth of nanoparticle penetrance into tissues and confirmation of drug release rates in tissues remain relatively unconfirmed. With rationale supporting the role of camptothecins in prostate cancer, potentially enriched on the basis of biomarker expression (e.g. TMPRSS2-ERG fusion [Chapter 4]), it is reasonable to explore optimized formulations. The recent introduction of trastuzumab deruxtecan ${ }^{16}$ provides rationale for developing a camptothecin-based antibody-drug conjugate targeting a patient-specific cell surface receptor, such as prostate specific membrane antigen (PSMA). ${ }^{17}$ Similarly, antibody conjugation to liposomal formulations or perhaps even the NLG207 co-polymer could be explored to further ensure targeted delivery to tumors. ${ }^{18}$ The development of more elegant approaches to nanoparticle unfolding and drug release, such as a mechanism dependent on tumor cell specific intracellular enzyme interaction to facilitate unfolding and release as opposed to $\mathrm{pH},{ }^{19}$ may also contribute to more stable nanoparticles in plasma, enhanced tolerability, and improved drug delivery to tumors. 
Ultimately, the work of this thesis sought to evaluate the feasibility of NLG207 and enzalutamide to treat patients with mCRPC following disease progression on enzalutamide. Over the course of numerous clinical trials preceding the current phase 2 studies at the NCI, the effectiveness of NLG207 has been mixed at best. A phase II trial enrolling 157 patients with non-small cell lung cancer (NSCLC) after one or two lines of chemotherapy showed reduced overall survival associated with NLG207 treatment in comparison to best supportive care (NCT01380769). In a phase 2 study enrolling 111 patients with metastatic renal cell carcinoma (mRCC) previously treated with 2-3 lines of chemotherapy, no differences in progression-free survival were observed when comparing NLG207 in combination with bevacizumab to standard of care treatment. ${ }^{20}$ It is reasonable to suggest inadequacies in trial design, especially with respect to the mRCC study, which potentially did not account for interplay of HIF-1 $\alpha$ and HIF- $2 \alpha$ facilitating tumorigenesis in the presence of sole HIF-1 $\alpha$ inhibition. ${ }^{21}$ Other smaller studies ( $\sim 30)$ have demonstrated the clinical benefit of NLG207, including its use in combination with capecitabine and radiotherapy in patients with locally advanced rectal cancer, ${ }^{22}$ and in combination with paclitaxel in patients with recurrent or persistent epithelial ovarian, fallopian tube or primary peritoneal cancer. ${ }^{23}$ Efficacy aside, a critical limitation of NLG207 treatment is the prolonged hydration strategy surrounding each dose implemented to mitigate non-infective cystitis, to which low grade bladderassociated toxicity is still observed despite its implementation (Chapter 7). ${ }^{14}$ Another major disadvantage of NLG207 therapy is the need for longer infusion times and a relatively short dosing interval. The ongoing trial will continue to address both the efficacy and limitations of NLG207 therapy. 
Irrespective of NLG207 development, the underlying important aim of this thesis work was to initiate the evaluation of dual HIF-1 $\alpha$ and AR inhibition in enzalutamide resistant tumors with respect to biomarkers of the AR pathway and angiogenesis. Also important to the efficacy evaluation is the contribution of topoisomerase I inhibition of camptothecin, which is also accounted for in the correlative biospecimen collection to evaluate effects on the DDR pathway (Chapter 7). Interpretation of biomarker signatures will be critical to confirm the anti-tumor activity observed in preclinical study, especially in a patient population with significant tumor heterogeneity unable to be re-capitulated by xenograft models (Chapters 2 \& 4). Biomarker signatures could also provide rationale for evaluation of more optimized camptothecin formulations and potent, specific inhibitors of HIF-1 $\alpha$ likely to come following continued drug development. Lastly, clinical results unknown at study initiation could provide a new hypothesis worthy of laboratory and/or clinical investigation.

The work presented in this thesis represents a small contribution to a much larger oncology community-based effort to optimize the treatment of advanced prostate cancer in response to SG-ARA resistance. In essence, the work provides a "snapshot" of the drug development process, capturing the steps necessary for generating an optimized clinical hypothesis from the lessons of negative trials. In facilitating the design of a clinical trial, the work emphasizes the importance of understanding key characteristics of the target treatment population, generating thorough scientific rationale via preclinical evaluation, and developing tools for the assessment of clinical questions specific to investigational treatment (e.g. pharmacokinetics). Importantly, the trial design adequately addresses the high failure rate of phase II studies, providing the potential for 
follow-up biospecimen analysis to re-initiate the translational research process

irrespective of achieving the primary study objective. These data did provide rationale

for implementing a nanoparticle-based approach to target HIF-AR crosstalk, but ultimately, it is the data generated beyond this work that will continue to drive drug development and further optimize the clinical management of acquired resistance to SGARA therapy.

References:

1. Fizazi K, Jones R, Oudard S, et al. Phase III, randomized, double-blind, multicenter trial comparing orteronel (TAK-700) plus prednisone with placebo plus prednisone in patients with metastatic castration-resistant prostate cancer that has progressed during or after docetaxel-based therapy: ELM-PC 5. J Clin Oncol 2015;33:723-31.

2. Taplin ME, Antonarakis ES, Ferrante KJ, et al. Clinical factors associated with AR-V7 detection in ARMOR3-SV, a randomized trial of galeterone (Gal) vs enzalutamide (Enz) in men with AR-V7+ metastatic castration-resistant prostate cancer (mCRPC). J Clin Oncol 2017;35:5005-.

3. Fizazi K, Shore N, Tammela TL, et al. Darolutamide in Nonmetastatic, Castration-Resistant Prostate Cancer. N Engl J Med 2019;380:1235-46.

4. Li Y, Yang R, Henzler CM, et al. Diverse AR Gene Rearrangements Mediate Resistance to Androgen Receptor Inhibitors in Metastatic Prostate Cancer. Clin Cancer Res 2020.

5. Robinson D, Van Allen EM, Wu YM, et al. Integrative clinical genomics of advanced prostate cancer. Cell 2015;161:1215-28.

6. Fernandez EV, Reece KM, Ley AM, et al. Dual targeting of the androgen receptor and hypoxia-inducible factor 1alpha pathways synergistically inhibits castration-resistant prostate cancer cells. Mol Pharmacol 2015;87:1006-12.

7. Yu T, Tang B, Sun X. Development of Inhibitors Targeting Hypoxia-Inducible Factor 1 and 2 for Cancer Therapy. Yonsei Med J 2017;58:489-96.

8. Jeong W, Rapisarda A, Park SR, et al. Pilot trial of EZN-2968, an antisense oligonucleotide inhibitor of hypoxia-inducible factor-1 alpha (HIF-1alpha), in patients with refractory solid tumors. Cancer Chemother Pharmacol 2014;73:343-8.

9. Lee K, Qian DZ, Rey S, Wei H, Liu JO, Semenza GL. Anthracycline chemotherapy inhibits HIF-1 transcriptional activity and tumor-induced mobilization of circulating angiogenic cells. Proc Natl Acad Sci U S A 2009;106:2353-8. 
10. Zamorano JL, Lancellotti P, Rodriguez Munoz D, et al. 2016 ESC Position Paper on cancer treatments and cardiovascular toxicity developed under the auspices of the ESC Committee for Practice Guidelines: The Task Force for cancer treatments and cardiovascular toxicity of the European Society of Cardiology (ESC). Eur Heart J 2016;37:2768-801.

11. Kummar S, Raffeld M, Juwara L, et al. Multihistology, target-driven pilot trial of oral topotecan as an inhibitor of hypoxia-inducible factor-1alpha in advanced solid tumors. Clin Cancer Res 2011;17:5123-31.

12. Thomas A, Pommier Y. Targeting Topoisomerase I in the Era of Precision Medicine. Clin Cancer Res 2019.

13. Conley SJ, Baker TL, Burnett JP, et al. CRLX101, an investigational camptothecin-containing nanoparticle-drug conjugate, targets cancer stem cells and impedes resistance to antiangiogenic therapy in mouse models of breast cancer. Breast Cancer Res Treat 2015;150:559-67.

14. Weiss GJ, Chao J, Neidhart JD, et al. First-in-human phase $1 / 2$ a trial of CRLX101, a cyclodextrin-containing polymer-camptothecin nanopharmaceutical in patients with advanced solid tumor malignancies. Invest New Drugs 2013;31:986-1000. 15. Clark AJ, Wiley DT, Zuckerman JE, et al. CRLX101 nanoparticles localize in human tumors and not in adjacent, nonneoplastic tissue after intravenous dosing. Proc Natl Acad Sci U S A 2016;113:3850-4.

16. Keam SJ. Trastuzumab Deruxtecan: First Approval. Drugs 2020;80:501-8.

17. Jones W, Griffiths K, Barata PC, Paller CJ. PSMA Theranostics: Review of the Current Status of PSMA-Targeted Imaging and Radioligand Therapy. Cancers (Basel) 2020;12.

18. Xu S, Cui F, Huang D, et al. PD-L1 monoclonal antibody-conjugated nanoparticles enhance drug delivery level and chemotherapy efficacy in gastric cancer cells. Int J Nanomedicine 2019;14:17-32.

19. de la Rica R, Aili D, Stevens MM. Enzyme-responsive nanoparticles for drug release and diagnostics. Adv Drug Deliv Rev 2012;64:967-78.

20. Voss MH, Hussain A, Vogelzang N, et al. A randomized phase II trial of CRLX101 in combination with bevacizumab versus standard of care in patients with advanced renal cell carcinoma. Ann Oncol 2017;28:2754-60.

21. Schodel J, Grampp S, Maher ER, et al. Hypoxia, Hypoxia-inducible Transcription Factors, and Renal Cancer. Eur Urol 2016;69:646-57.

22. Sanoff HK, Moon DH, Moore DT, et al. Phase I/II trial of nano-camptothecin CRLX101 with capecitabine and radiotherapy as neoadjuvant treatment for locally advanced rectal cancer. Nanomedicine 2019;18:189-95.

23. Duska L, O'Malley DM, Krasner C, et al. Abstract CT151: A Phase II study of NLG207 (formerly CRLX101) in combination with weekly paclitaxel in patients with recurrent or persistent epithelial ovarian, fallopian tube or primary peritoneal cancer. Cancer Res 2019;79:Suppl. 


\section{Acknowledgements}

I am grateful and appreciative to the numerous people who have participated in my education, supported me and have guided my career thus far.

Thank you to my promoter, Prof. dr. Alwin D. R. Huitema, for assuming this important role in my mentorship and navigating me to the completion of this crowning achievement in my education. I am incredibly thankful to have received your frequent counsel throughout working on this collaborative thesis, despite being over $6,000 \mathrm{~km}$ away.

Thank you to the Department of Pharmacy and Pharmacology at the Netherlands Cancer Institute, including Prof. dr. Jos Beijnen, Dr. Thomas P. C. Dorlo, and Thea Eggenhuizen, for graciously hosting me in Amsterdam while working on this collaborative thesis. I learned much during my time in the Netherlands and will remember my experience at the NKI fondly for years to come.

Thank you to my co-promoter, Dr. William D. Figg, for your mentorship on this collaborative thesis project and guidance in my career trajectory. When I first met you as a rotating pharmacy student, I was uncertain about pursuing a thesis project. It was the early conversations with you and the amazing opportunities at the National Cancer Institute that made this important career decision a "no-brainer," and I haven't looked back since. Thank you for continuing to challenge me intellectually, you have made me both a better scientist and a better person!

Thank you to Dr. Cody J. Peer for being not only a patient, committed mentor to me, but also a role model as a diligent, efficient, and motivated scientist. You have been a key advocate in my education by "showing me the ropes" of clinical pharmacokinetic analyses and emboldening my ability to develop concise, focused scientific arguments. I have always appreciated the time and effort you have taken to mentor me over the years.

Thank you to Dr. Cindy H. Chau for always being available to discuss dire questions about almost anything from an experiment gone wrong to the best approach for clinical biospecimen collection. Your expansive expertise and insistence to promote my independence as a researcher has been invaluable to me. I have greatly benefited from your approach to mentorship and enthusiasm for research.

Thank you to Dr. Tristan M. Sissung for your advice on many facets of this collaborative thesis, including recommendations for many experiments and the selection of appropriate statistical tests for datasets. I have appreciated your willingness to engage in frequent scientific debate, as it has not only improved the quality of this thesis work but has also helped me grow as a scientist. 
Thank you to Dr. Douglas K. Price for being one of my biggest champions throughout my time at the National Cancer Institute. When things didn't go according to plan, your door was always open to talk things through. When I succeeded, you were always one of the first people to congratulate me. Thank you for being there through the ups and downs over the years.

Thank you to the clinical team of the Genitourinary Malignancies Branch of the Center for Cancer Research at the NCI, especially Drs. Ravi A. Madan, Lisa M. Cordes, Fatima Karzai, Marijo Bilusic, James L. Gulley, and William L. Dahut, for facilitating my participation in the prostate cancer clinic over the years. You have all contributed greatly to my professional development as a pharmacist and cancer researcher. It has been an honor and privilege to work with such a cordial and dedicated clinical team.

Thank you to all members of the Figg Laboratory, past and present, and all collaborators involved with the work of this collaborative thesis. This was indeed a collaborative venture and would not have been possible without the combined talents of all of you.

Thank you to the wonderful friends I have met during my time as a graduate student at NIH, especially Nicholas Sciascia, Rachel Burga, Keyla Tumas, Carly Starke, and Kristoffer Haurum Johansen. I have been so fortunate to have an incredible support group to not only discuss the rigors of graduate student life, but to also spend free time with outside of the laboratory.

Thank you to two of my closest friends from my years in Chapel Hill, Chad Walker and David Singer, who were a great source of encouragement to me during my pharmacy school education and over long distance during the course of this collaborative thesis project. Your friendship has been a motivating presence in my life and I'm thankful we still remain in touch after all this time.

Thank you to Helen Cuffe for being there to encourage and support me during the final year of my thesis work. You have been such a positive influence and I am so thankful to have you in my life.

Lastly, thank you to my parents, Richard and Karen Schmidt, for always supporting me throughout my academic endeavors. It is hard to believe it has been 10 years since I first told you my intention to earn dual doctoral degrees when I was first applying to pharmacy school. Though quite a lofty undertaking to pursue, you both somehow knew this day would come, even on the days when I doubted myself. Thank you for always instilling in me a motivation to go beyond expectations, this achievement does not happen without you! 


\section{Curriculum Vitae}

The author of this thesis was born in New Hyde Park, New York, USA on August 4, 1989. In 2007, he moved to Chapel Hill, North Carolina and completed his Bachelor of Science degree in Chemistry and Biology at the University of North Carolina in 2011. In 2015, he completed his Doctor of Pharmacy degree at the Eshelman School of Pharmacy of the University of North Carolina and subsequently received licensure to practice pharmacy in the states of North Carolina and Maryland. Since 2015, he has worked within the Clinical Pharmacology Program headed by Dr. William D. Figg at the Center for Cancer Research of the National Cancer Institute in Bethesda, Maryland. Research has included that contained in this thesis, in addition to in vitro, preclinical and clinical studies on a range of cancer therapeutics and biomarkers, with particular emphasis on the improvement of prostate cancer standard of care treatment. The work presented in this thesis originated from the Figg Laboratory and collaboration with Prof. dr. Alwin D. R. Huitema of the Department of Pharmacy and Pharmacology at the Netherlands Cancer Institute in Amsterdam and Department of Clinical Pharmacy of the University Medical Center Utrecht, both in the Netherlands. 


\section{Publications}

Related to thesis work:

1. Schmidt KT, Peer CJ, Huitema ADR, Williams MD, Wroblewski S, Schellens JHM, Madan RA, Figg WD. Measurement of NLG207 (formerly CRLX101) nanoparticle-bound and released camptothecin in human plasma. $J$ Pharm Biomed Anal. 2020 Mar 20;181:113073.

2. Madan RA, Schmidt KT, Karzai F, Peer CJ, Cordes LM, Chau CH, Steinberg SM, Owens H, Eisner J, Moore WR, Dahut WL, Gulley JL, Figg WD. Phase 2 Study of Seviteronel (INO-464) in Patients With Metastatic Castration-Resistant Prostate Cancer After Enzalutamide Treatment. Clin Genitourin Cancer. 2020 Aug;18(4):258-267.

3. Schmidt KT, Huitema ADR, Dorlo TPC, Peer CJ, Cordes LM, Sciuto L, Wroblewski S, Pommier Y, Madan RA, Thomas A, Figg WD. Population Pharmacokinetic Analysis of Nanoparticle-bound and Free Camptothecin after Administration of NLG207 in Adults with Advanced Solid Tumors. Cancer Chemother Pharmacol. In Press.

4. Schmidt KT, Huitema ADR, Chau CH, Figg WD. Precision Medicine for $2^{\text {nd }}$ Generation Androgen Receptor Antagonist Resistance in Prostate Cancer. Submitted

5. Schmidt KT, Chau CH, Strope JD, Huitema ADR, Sissung TM, Price DK, Figg WD. Anti-tumor activity of NLG207 (formerly CRLX101) in combination with enzalutamide in preclinical prostate cancer models. Submitted

Unrelated to thesis work:

1. Handy WF, Schmidt KT, Price DK, Figg WD. Examining HSD3B1 as a possible biomarker to detect prostate cancer patients who are likely to progress on ADT. Cancer Biol Ther. 2020 Aug;13:1-3

2. Knechel MA, Schmidt KT, Figg WD. Combination treatment in metastatic castration-resistant prostate cancer: can we safely boost efficacy by adding radium-223? Cancer Biol Ther. 2020;21(1):1-3

3. Peer CJ, Schmidt KT, Kindrick JD, Eisner JR, Brown VV, Baskin-Bey E, Madan R, Figg WD. A population pharmacokinetic analysis of the oral CYP17 lyase and androgen receptor inhibitor seviteronel with advanced/metastatic castration-resistant prostate cancer or breast cancer. Cancer Chemother Pharmacol. 2019 Oct;84(4):759-770 
4. Sissung TM, Schmidt KT, Figg WD. Insulin potentiation therapy for cancer? Lancet Oncol. 2019 Feb;20(2):191-192

5. Karakunnel JJ, Bui N, Palaniappan L, Schmidt KT, Mahaffey KW, Morrison B, Figg WD, Kummar S. Reviewing the role of healthy volunteer studies in drug development. J Transl Med. 2018 Dec 4;16(1):336

6. Schmidt KT, Madan RA, Figg WD. Expanding the use of abiraterone in prostate cancer: Is earlier always better? Cancer Biol Ther. 2018 Feb 1;19(2):97-100.

7. Kaniskan HU, Eram MS, Zhao K, Szewczyk MM, Yang X, Schmidt K, Luo X, Xiao S, Dai M, He F, Zang I, Lin Y, Li F, Dobrovetsky E, Smil D, Min SJ, LinJones J, Schapira M, Atadja P, Li E, Barsyte-Lovejoy D, Arrowsmith CH, Brown PJ, Liu F, Yu Z, Vedadi M, Jin J. Discovery of Potent and Selective Allosteric Inhibitors of Protein Arginine Methyltransferase 3 (PRMT3). J Med Chem. 2018 Feb 8;61(3):1204-1217.

8. Newsome AS, Sultan S, Murray B, Jones SW, Pappas A, Schmidt KT, Filteau G, Laux JP, Wolfe A, Williams F, Cairns BA. Effect of inhaled iloprost on gas exchange in inhalation injury. Burns Open. 2017 Oct;1(2):49-53.

9. Schmidt KT, Figg WD. The potential role of curcumin in prostate cancer: the importance of optimizing pharmacokinetics in clinical studies. Transl Cancer Res. 2016 Nov;5(Suppl 6):S1107-S1110.

10. Schmidt KT, Chau CH, Price DK, Figg WD. Precision Oncology Medicine: The Clinical Relevance of Patient-Specific Biomarkers Used to Optimize Cancer Treatment. J Clin Pharmacol. 2016 Dec;56(12):1484-1499.

11. Kaniskan HU, Szewczyk MM, Yu Z, Eram MS, Yang X, Schmidt K, Luo X, Dai M, He F, Zang I, Lin Y, Kennedy S, Li F, Dobrovetsky E, Dong A, Smil D, Min SJ, Landon M, Lin-Jones J, Huang XP, Roth BL, Schapira M, Atadja P, Barsyte-Lovejoy D, Arrowsmith CH, Brown PJ, Zhao K, Jin J, Vedadi M. A potent, selective and cell-active allosteric inhibitor of protein arginine methyltransferase 3 (PRMT3). Angew Chem Int Ed Engl. 2015 Apr 20;54(17):5166-5170. 